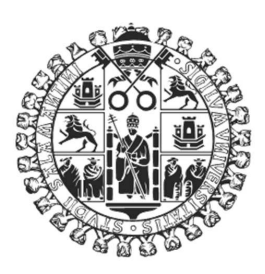

Universidad de Salamanca

Facultad de Ciencias

Departamento de Física Aplicada

\title{
Modeling spin transfer induced oscillations in spin valve nanostructures.
}

Elżbieta Jaromirska

December 2010 

D. Luis López Díaz, Profesor Titular de Electromagnetismo y miembro del Departamento de Física Aplicada de la Universidad de Salamanca

\section{CERTIFICA}

Que la presente Memoria, titulada Modeling spin transfer induced oscillations in spin valve nanostructures y presentada por D. Elżbieta Jaromirska para optar al Grado de Doctora en Física, ha sido realizada en su totalidad bajo su dirección en el Área de Electromagnetismo del Departamento de Física Aplicada de la Universidad de Salamanca.

Salamanca, 10 de diciembre de 2010

Fdo. Luis López Díaz

Profesor Titular de Electromagnetismo

Universidad de Salamanca 


\section{LIST OF ABBREVIATIONS}

$\begin{array}{lll}\text { ABC } & - & \text { Absorbing Boundary Conditions } \\ \text { AP } & - & \text { AntiParallel state } \\ \text { CIMS } & - & \text { Current Induced Magnetic Switching } \\ \text { CMOS } & - & \text { Complementary Metal Oxide Semiconductor } \\ \text { Co } & - & \text { Cobalt } \\ \text { CPP } & - & \text { Current Perpendicular to Plane } \\ \text { Cu } & - & \text { Copper } \\ \text { cw } & - & \text { clockwise } \\ \text { ccw } & - & \text { counterclockwise } \\ \text { DRAM } & - & \text { Dynamic Random Access Memory } \\ \text { F } & - & \text { Ferromagnetic layer } \\ \text { FL } & - & \text { Free Layer } \\ \text { FTT } & - & \text { Fast Fourier Transform } \\ \text { GMR } & - & \text { Giant MagnetoResistance } \\ \text { ICF } & - & \text { Interlayer Coupling Field } \\ \text { IP } & - & \text { In-Plane } \\ \text { IPP } & - & \text { In-Plane Precession } \\ \text { LLG } & - & \text { Landau-Lifshitz-Gilbert } \\ \text { MRAM } & - & \text { Magnetoresistive Random Access Memory } \\ \text { MTJ } & - & \text { Magnetic Tunnel Junction } \\ \text { N } & - & \text { Nonmagnetic layer } \\ \text { ODE } & - & \text { Ordinary Differential Equation } \\ \text { OP } & - & \text { Out-of-Plane }\end{array}$




$\begin{array}{lll}\text { OPP } & - & \text { Out-of-Plane Precession } \\ \text { P } & - & \text { Parallel state } \\ \text { PC } & - & \text { Point Contact } \\ \text { PL } & - & \text { Pinned Layer } \\ \text { Py } & - & \text { Permalloy } \\ \text { SAF } & - & \text { Synthetic AntiFerromagnet } \\ \text { SPC } & - & \text { Spin Polarized Current } \\ \text { ST } & - & \text { Spin Torque } \\ \text { STNO } & - & \text { Spin Torque Nano Oscillator } \\ \text { STT } & - & \text { Spin Transfer Torque } \\ \text { TMR } & - & \text { Tunneling MagnetoResistance }\end{array}$




\section{CONTENTS}

1. Introduction . . . . . . . . . . . . . . . . 1

1.1 Overview ....................... 1

1.2 This work . . . . . . . . . . . . . . . . . 4

1.3 Guide to this thesis . . . . . . . . . . . . . . . 9

2. Theoretical and computational techniques . . . . . . . . . 12

2.1 Magnetization dynamics . . . . . . . . . . . . . . 12

2.1.1 Precession . . . . . . . . . . . . . . 13

2.1.2 Damping . . . . . . . . . . . . . . . 15

2.2 Spin transfer in the diffusive transport limit . . . . . . . . . 16

2.2.1 Spin current and spin accumulation . . . . . . . . 16

2.2.2 Boundary conditions . . . . . . . . . . . . . 22

2.2 .3 Spin torque . . . . . . . . . . . . . . . . 23

2.3 Mesoscale models . . . . . . . . . . . . . . . . . . . 26

2.3.1 Macrospin model . . . . . . . . . . . . . . . 27

2.3.2 Micromagnetic model . . . . . . . . . . . . . . . 28

2.4 Computational techniques . . . . . . . . . . . . . . . . 32

2.4.1 Exchange energy . . . . . . . . . . . . . . . 33

2.4 .2 Anisotropy energy . . . . . . . . . . . . . 34

2.4.3 Self magnetostatic energy . . . . . . . . . . . . . 35

2.4.4 External field energy . . . . . . . . . . . . . . . 36

3. Spin torque driven oscillations in an asymmetric spin valve . . . . . 37

3.1 Specifications . . . . . . . . . . . . . . . . 37 
3.2 Critical angle study . . . . . . . . . . . . . . . . . . . . . 40

3.3 Oscillations in presence of external field. . . . . . . . . . . . . 45

3.3.1 Field orientation $0^{\circ}<\alpha<90^{\circ} \ldots \ldots \ldots . . \ldots 45$

3.3.2 Low fields; $\alpha>90^{\circ} \ldots \ldots . . . . . . . . .446$

3.3.3 Intermediate fields; $\alpha>90^{\circ} \ldots \ldots$. . . . . . . 48

3.3.4 Large fields; $\alpha>90^{\circ} \ldots \ldots \ldots \ldots$. . . . . . 50

3.4 Oscillations in absence of external field . . . . . . . . . . . . 51

3.4.1 Extended geometry, AP initial state . . . . . . . . . 53

3.4 .2 Etched geometry . . . . . . . . . . . . . 55

3.4 .3 Influence of the exchange field . . . . . . . . . . . 58

3.4.4 Comparison to experimental data . . . . . . . . 60

3.5 Conclusion . . . . . . . . . . . . . . . . . . 67

4. Vortex oscillations in single point contact geometry . . . . . . . . 68

4.1 Introduction to vortex mode . . . . . . . . . . . 68

4.2 Point contact geometry, computational issues . . . . . . . . . 70

4.2.1 Linear mode . . . . . . . . . . . . . . . . 71

4.2.2 Vortex mode. . . . . . . . . . . . . 75

4.3 Initial configuration in hybrid geometry . . . . . . . . . . 80

4.3.1 Sample characteristics and simulation parameters . . . 80

4.3.2 Geometry induced aspects . . . . . . . . . . . . 81

4.3.3 Current and Oersted field calculations . . . . . . . 83

4.3.4 Initial state in presence of currents . . . . . . . . . . 85

4.4 Current dependence . . . . . . . . . . . . . . . . . . . . 88

4.5 Field dependence . . . . . . . . . . . . . . . . . . . . . 95

4.6 Conclusion . . . . . . . . . . . . . . . . . . . . . . . . . . 101

5. Interlayer coupled vortices in single point contact . . . . . . . . . . 103

5.1 Introduction to double vortex oscillator. . . . . . . . . . . 103

5.1.1 Geometry and convention . . . . . . . . . . 104

5.1.2 Simulation parameters and methods . . . . . . . 106 
5.1.3 Nucleation and torques . . . . . . . . . . . . 107

5.2 Positive current . . . . . . . . . . . . . . . . . . . . . . 108

5.3 Negative current . . . . . . . . . . . . . . . . . . . 112

5.4 Systematic study, $\mathrm{Co}(5 \mathrm{~nm}) / \mathrm{Cu}(5 \mathrm{~nm}) / \mathrm{Py}(5 \mathrm{~nm}) \ldots 115$

5.4.1 Positive current . . . . . . . . . . . . . 116

5.4 .2 Negative current . . . . . . . . . . . . . . . . . . 119

5.4 .3 Results summary . . . . . . . . . . . . . . 128

5.4 .4 Two vortices of different polarity . . . . . . . . . . 131

5.5 Two equal ferromagnets . . . . . . . . . . . . . . . . 147

5.5.1 Common polarity . . . . . . . . . . . . . . . 148

5.5 .2 Opposite polarity . . . . . . . . . . . 156

5.6 Conclusions . . . . . . . . . . . . . . . . 165

6. Coupled vortices dynamics . . . . . . . . . . . . . . . . . . . 168

6.1 Experimental findings . . . . . . . . . . . . . . . 168

6.2 Current and Oersted field distribution . . . . . . . . . . . 169

6.3 Remarks . . . . . . . . . . . . . . . . . . . 172

7. Summary and outlook . . . . . . . . . . . . . . . . 173

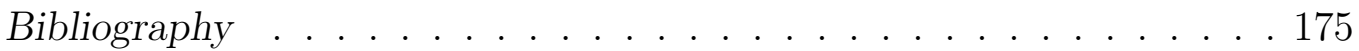

Acknowledgments . . . . . . . . . . . . . . . . . . . . . . 181 


\section{INTRODUCTION}

\subsection{Overview}

The rapid development of the modern natural sciences, engineering and medicine has been the main cause of the overall industrial and intellectual revolution the humanity has been experiencing over the past two centuries. The work you are about to read humbly pushes ahead the wheel of modern natural sciences by propelling it particular wheelarm called electromagnetism. Moreover, it adds a modest contribution to building the bridge to future application of spintronic devices. Spintronics (a neologism meaning spin transport electronics [1, 2]) is an emerging technology combining two twin fields of physics: electronics and magnetism. The first one is based on the existence of electric charge and the second on it magnetic property: spin. Spintronics takes, therefore, twofold advantage of the electron.

The birth of spintronics dates back to ferromagnet/superconductor tunneling experiments pioneered by Meservey and Tedrow [3] and the initial experiments on magnetic tunnel junctions (MTJ) by Julliere [4] in the 1970s. In the 1980s this investigation line was further explored by experiments on spindependent electron transport phenomena in solid state devices [5]. Though, the first turning point that attracted lots of attention of the scientific community and accelerated the progress of industrial magnetic devices was the discovery of giant magnetoresistance in 1988 (GMR) for which Albert Fert and Peter Grünberg were awarded the Nobel Prize (2007). This effect can be observed in a device consisting of two ferromagnetic layers separated by a nonmagnetic spacer where the resistance of the device depends on the rela- 
tive magnetization configuration of the adjacent ferromagnetic layers. Hence, if both are magnetized along common direction the resistance of the device is low. On the other hand, if the respective configuration is antiparallel the current applied experiences high resistance. This effect, translating the magnetic state of the element into voltage, found numerous applications in the sensor and information storage industry.

Another breakthrough came in 1996 when Slonczewski [6] and Berger [7] introduced the pioneering concept of spin transfer predicting a new way of manipulating the magnetic state of the sample by employing spin polarized current (SPC). It was shown theoretically that such current exerts a torque on a thin magnetic film due to transfer of spin angular momentum influencing the magnetic configuration of a ferromagnet. This discovery, together with the great progress in the nanotechnology, set new era in the spintronic research. During the next years new fabrication techniques brought about nanosize devices where combined effects of electron charge and spin could be observed confirming theoretical prediction.

In general, spin transfer torque (STT) manifests itself in the current induced magnetic switching (CIMS) $[8,9]$ and the generation of microwave oscillations $[10,11]$. Considering that in its first form, field controlled, magnetoresistive random access memory (MRAM) required large external fields in order to invert the magnetization throughout the sample volume its practical design encountered certain disadvantages i.e. in order to produce sufficiently strong fields very large currents had to be applied. Hence, the CIMS effect brought about the novel idea of current-controlled MRAM. On the other hand, this solution not only decreases the power consumption but also reduces the read and write times making it a viable non-volatile alternative to DRAM and FLASH memory technologies. Along with its high efficiency, the ability of retaining data, even if power is lost, makes current-switched MRAM subject to thorough study these days but industrial ST MRAM is yet to be delivered. 
The second spin torque (ST) driven phenomenon is the steady state precession, which by means of GMR or TMR (tunneling magnetoresistance) converts magnetization changes into voltage variations. This provides the functional base for the design of $\mathrm{GHz}$ and sub-GHz range current-controlled oscillators that could be used in mobile wireless communications or novel logic and signal processing applications. The main advantage of such spin torque nanooscillator (STNO) is its small size and possibility of integration with the standard CMOS (Complementary Metal Oxide Semiconductor) transistor technology. The application tailored design and optimization are still a challenge to scientists and engineers since low output power ( $\mathrm{pW}$ and $\mathrm{nW}$ ) and broad linewidths have to be improved before a reliable and cheap STNO is on market. Lately, the increase of power was demonstrated by coupling multiple oscillators $[12,13]$ but mechanisms that lead to coupling are not very well understood yet. Therefore, proposing a better and more reliable solution is a challenge yet to be taken.

On the other hand, the struggle to reduce the linewidth is also ongoing $[14,15]$. As has been reported in [14] the synchronization of multiple modes oscillating in the common ferromagnetic mesa might also lead to narrower linewidths. The second ferromagnetic layer serves as the reference. The design of sample used in this case is called the point contact geometry (PC). As the name indicates the current is confined while it is injected to the top of the magnetic layer via nanosize etched hole in the insulating layer.

In parallel, the linewidth problem has been approached by investigating the devices with pillar geometry where the current spreads across the entire cross section of the nanosize pillar. The novel idea of allowing both ferromagnetic layers of a MTJ to evolve dynamically demonstrated that modes excited in each layer can be coupled to each other resulting in great improvement of the linewidth [15]. The major drawback of this solution is that the absence of the reference layer significantly reduces the output power. Both cited experiments were carried out in the current perpendicular to plane 
(CPP) geometry assuring large current densities required to observe STT effect. Second design aimed at different application is the current in-plane (CIP) which is, however, beyond the subject of this thesis.

After this introduction the reader should be aware of all the obstacles the spintronics community is struggling with. Almost one and a half decade after the discovery of STT, the state-of-the-art SPC driven devices are still only laboratory prototypes and not industrial reality. As for the memory applications, the possibilities brought about by CIMS have not been fully exploited and the ST MRAM is yet to be delivered. STNO are somewhere further on their way to the factory production line but still more understanding of physical processes behind is required. Therefore, this thesis pointing along these lines, is dedicated to the investigation of SPC driven magnetic oscillators.

\subsection{This work}

This section points out the general lines of the work presented within. Also for less familiar readers a broad introduction to the up to date achievements in spintronics and state-of-the-art experiments is provided.

In general, this work is dedicated to the numerical study of the current induced magnetization oscillations in spin valves. From the application point of view oscillators working at low, or even in absence of external field are of particular interest. Even though the existence of such oscillators has been predicted theoretically [16] and demonstrated experimentally [17, 18, 19, 14] there is a huge gap to be filled out with the numeric modeling providing the basic understanding of predicted and/or observed tendencies. Therefore, in the following an exhaustive systematic modeling is carried out revealing details about little known physical processes behind reported trends. The results of this study are compared to the experimental data yielding qualitative explanation of features governing magnetization dynamics. 
First, the quasi-uniform magnetization precession in pillar geometry with nonstandard ST profile is investigated by means of macrospin and micromagnetic simulations. Later, gyrating vortex mode in the PC geometry is studied. However, this geometry and this mode are both beyond macrospin approximation and, therefore, only full micromagnetic study provides the correct description of the observed dynamics. The common feature of two different oscillators studied within this work is the possibility, at least in principle, to work in absence of external field.

Since the work deals with SPC one should start with brief introduction to STT and models that have been developed for its calculation up to now. From the practical point of view, considering that STT originates from spin asymmetries of the two independent transport channels, its properties are related to device design and materials. Hence, STT and CPP-GMR phenomena are inseparably correlated [20] and depend on the same structural parameters. First, Valet and Fert [21] incorporated most of them into a model, considering two independent conducting electron channels which obey Boltzman diffusive transport equation. Further generalization of Valet-Fert model [16] provided an unified description of STT and CPP-GMR in diffusive transport limit. On the other hand, the torque modeled within Slonczewski framework [6] was based on ballistic assumptions and is to be called standard torque throughout this work.

For symmetric spin valves, with both ferromagnetic layers made of the same material, such as $\mathrm{Co} / \mathrm{Cu} / \mathrm{Co}$, the diffusive model predicts standard STT, which does not vary qualitatively from Slonczewski's results [6]. In this case, flowing electrons drive CIMS [22]. Moreover, the generation of microwave oscillations is possible only in presence of applied fields higher than the coercive field [11]. This STT-driven behavior has been recently observed in $\mathrm{Py} / \mathrm{Cu} / \mathrm{Py}$ (Py = Permalloy) experimentally [23, 24] and numerically [25]. Also an asymmetric structure $\mathrm{Co}(40 \mathrm{~nm}) / \mathrm{Cu}(10 \mathrm{~nm}) / \mathrm{Py}(3 \mathrm{~nm})$, where the free and the pinned layer are made of different material, might exhibit this 
behavior as a consequence of the layer thickness ratio supporting standard torque as reported in [26].

However, it has been shown that in an asymmetric pillar geometry, where layer thickness obeys certain conditions and bulk and interface asymmetry factor and spin diffusion length are different in each magnetic film, an anomalous, wavy-like, dependence of STT on the angle between magnetization vectors can be observed [16]. The STT vanishes and changes its sign in a certain noncollinear magnetic configuration because of the appearance of an inverse spin accumulation in the nonmagnetic spacer. Therefore, on the contrary to standard STT, which stabilizes one of the collinear magnetic configurations and destabilizes the opposite one, wavy torque stabilizes both of them for certain current direction and destabilizes both of them for the opposite direction. The latter is of particular interest as it leads to excitation of oscillation modes in absence of any external field. Asymmetric structures have been recently thoroughly investigated theoretically $[16,27,28]$ as well as experimentally at low and zero applied field [17, 18]. Nevertheless, the wavy torque-induced magnetization dynamics has been studied so far only in the macrospin approximation $[29,30]$ which does not describe correctly the dynamics at low applied magnetic field [18] and is, thus, not able to reproduce the experimental observations completely. Therefore, part of this work is dedicated to the calculation of the STT in the diffusive transport limit and comparison of the systematic numerical study to the experimental findings $[31,32]$.

Understanding magnetization dynamics requires development of mathematical models describing real processes observed in nature. The theory of micromagnetism, in which the magnetization is described as a space dependent continuous field [33], is the appropriate framework to describe magnetization on the nanoscale and will be used throughout this work. On the other hand, so-called macrospin model, in which the magnetization of each layer is treated as a single spin, is often used. Providing much simpler description of 
the system it might lead to general understanding of the observed features. Thus, before comparing results emerging from both models, one should realize that the concept of single domain magnetic particle in many situations is simply invalid. In principle, the exchange energy density of a closed $\boldsymbol{M}(\boldsymbol{r})$ configuration increases as the particle size decreases and this could justify macrospin approach below certain critical size of the system even though spin torque makes the estimation of this critical size difficult [34]. However, as reported in [35] the steady state precession of a thin square nanoelement exhibits complicated transition from quasimacrospin to chaotic behavior already at the size of $30 \mathrm{~nm}$, which invalidates single domain approximation for most of experimentally studied systems. Moreover, Berkov and Gorn [36] micromagnetically identified some artifacts of macrospin model in the ballistic transport limit. These artifacts might cause misleading interpretation of the origin of observed phenomena [11].

In this work the reader will find a comparison of the predictions of both micromagnetic and macrospin models for a particular problem i.e. current induced oscillations in an asymmetric spin valve in the diffusive transport limit. Certain differences will be pointed out.

The remaining part of this work focuses on the PC devices, which represent a class of experimental systems where macrospin approximation is never valid. In PC geometry only full micromagnetic analysis can be applied for the description of magnetization dynamics. Moreover, highly nonuniform modes like a magnetic vortex studied within this work are also beyond single spin approximation. Therefore, in Chapter 4 and thereafter numerical study will refer to the micromagnetic study only.

A challenging example of low frequency magnetic vortex mode in PC device is going to be investigated. First traces of low frequency oscillations have been reported in Ref. [37] but first convincing evidence of ST induced dynamics of magnetic vortex was provided in constrained pillar geometry [38] and later in the extended PC device [39]. 
Vortex mode has raised strong interest because of its unique characteristics. First of all, the oscillations can be sustained in absence of external field [19] at relatively low current densities. Moreover larger power outputs $(\mathrm{nW})$ are obtained together with narrower linewidths as compared to other modes. Numerous theoretical models [40, 39, 41] along with numerical and experimental studies [42] have characterized vortex mode. First, it has been shown that the out-of-plane component of the magnetization in the PL is essential for the vortex to be excited into steady motion $[39,43]$. Later it has been demonstrated that also a nonuniform in-plane magnetized polarizer [44] can give rise to current induced vortex dynamics. Still, in all of the studies so far the polarizer has been assumed to be static in time, which in many experiments was fulfilled by introducing exchange bias or SAF to the PL. Moreover, it was long believed that the vortex state is only stable in layers of certain thickness $[45,14]$. This work shows that this assumption requires verification when ST driven dynamics is considered.

Aiming at comparison with experimentally reported but poorly understood behavior, a systematic study of a vortex mode in a single PC device has been carried out leading to discovery of new features of this mode. In particular, it has been shown that in a system without exchange bias, the geometry itself assists ST in expulsion of vortex from below the PC area. Therefore, not only the STT effect as believed up to now, but also inhomogeneous magnetostatic fields, present in the sample as a effect of its geometry might play crucial role in the excitation of vortex mode. An excellent quantitative agreement between numerical and measured data has been found leading to a qualitative explanation of the observed trends.

Another interesting and so far little studied system is a spin valve with two active ferromagnetic layers $[46,15]$. It has been demonstrated in pillar geometry [15] that the coupling between the vortices nucleated one in each magnetic layer leads to very narrow linewidths. However, no study of such vortex pair in PC geometry has been performed so far and, therefore, further 
part of this work is dedicated to exploring this new and promising subject. The results of this numerical consideration might be of help to experimentalists designing PC based double vortex STNO.

Other milestone, as far as synchronization in PC devices is concerned, was reached by Ruotolo et al. [14] by proposing an array of four point contacts opened in a common ferromagnetic film by means of nano-indentation technique [47]. The unique property of this system, called hereafter hybrid, is the non flat profile of the thick layer. The power reported in this system was found to be field dependent exhibiting its highest value at $0 \mathrm{mT}$. Additionally, the sample behavior was shown to be history dependent, i.e. application of small in-plane fields in the nucleation process hindered the appearance of the single spectral peak. Although very convincing experimental evidence of synchronization was provided the understanding of the reported trends is still far from being complete. The explanation provided in [14] is rather confusing and definitely insufficient. Already the definition of the active layer and the initial state, determined in the last Chapter of this thesis, proved not to be compatible with [14]. Therefore, despite the technical difficulties this particular geometry introduces, the final part of this work is dedicated to study of such multiple contact system.

\subsection{Guide to this thesis}

This thesis presents computational study of different low field STNO. Therefore, some parts of this work might be of interest to the reader seeking theoretical bases of the STT effect (Chapter 2). Other parts are rather useful for an experimentalist searching for the explanations of observed trends (Chapter 3, 4, 6) and/or fabricating the spin valve with a design for particular applications (Chapter 5). Moreover, the numeric difficulties encountered when studying vortex mode in PC devices would be interesting for the reader rather familiar with the micromagnetic simulations (Chapter 4). Therefore, 
this guide is presented in order to define the particular interest of each section.

Chapter 2 derives the dynamic equation of magnetization motion starting with the quantum mechanical counterpart for a free electron. This dynamic equation is then supplemented with the diffusive ST term whose derivation based on model [16] is carried out. Numerical implementation of the ST into the dynamic equation required the development of an application in Mathematica [48] for the calculation of ST term, which has been carried out in collaboration with Pavel Baláž and Prof. Józef Barnaś from Adam Mickiewicz Univeristy, Poznan (Poland). Later in the chapter numerical modeling of magnetization dynamics and its technical aspects are presented for the macrospin and micromagnetic models together with the limitations involved.

Chapter 3 takes advantage of the ST derivation from previous chapter and applies it for the computational study of a STNO pillar at low field. Both mesoscopic models are employed for the modeling of magnetization dynamics driven by the wavy torque. The results are compared to the experimentally reported trends. Very good quantitative match is reported yielding qualitative explanation of observed features.

Chapter 4 is dedicated to the vortex based oscillator in a single PC geometry. A complicated system fabricated by means of nano-indentation technique is considered. The frequency dependence on both current and field observed in a real device were matched quantitatively with the computational results. Moreover, qualitative explanation of observed phenomena led to the discovery of novel way of tailoring vortex-based STNO. This part of work was carried out in collaboration with the experimental group CNRS/Thales from Palaiseau (France).

Chapter 5 presents the results of investigation of a double vortex mode. An interlayer-coupled oscillator in a single PC geometry is studied i.e. both ferromagnetic layers were allowed to evolve dynamically which led to nu- 
cleation of two vortices, one in each layer. This mode was first described qualitatively by indicating the differences in the frequency of the GMR signal and the radii of the vortex orbits in both ferromagnets arising from the location of ST active interfaces. In particular, limiting cases of ST acting only on the top and only on the bottom layers were studied. The quantitative analysis of this system was also carried out leading to predictions that will certainly be of interest for the future experimental study of double vortex STNO, in particular the design of the spin valve structure itself for this novel application.

Chapter 6 deals with the analysis of a multiple contact system. The complicated geometry of the valve and the complexity of the synchronization mechanism itself in such sample presented a great challenge to the possibilities of the state-of-the-art hardware and software resources. Despite these difficulties some preliminary study of phase locking was carried out.

Finally the conclusions are presented in Chapter 7 along with the future outlook on the research in the field of spintronic oscillators. 


\section{THEORETICAL AND COMPUTATIONAL TECHNIQUES}

This chapter serves as an introduction to the physical fundamentals of the magnetization dynamics and SPC associated phenomena. Their interpretation requires modeling on the level of the nanoscale. In the following such models are described, their validity range is specified and their limitations are pointed out. Moreover, some details with respect to the computational techniques are given.

\subsection{Magnetization dynamics}

The purpose of this section is to provide basic description of magnetization dynamics starting from the quantum mechanical counterpart for a free electron spin [49].

A precessional state is obtained as a solution of such problem. However, in reality precessional states observed in presence of conservative fields tend to adapt to a final remanent state. Therefore, to fit experimental observations and theoretical predictions the phenomenological damping had to be introduced. Finally, an interesting effect of sustaining oscillatory states by means of counterbalancing such damping is introduced. This phenomenon is called spin transfer effect and it is derived from transport theory. 


\subsubsection{Precession}

In the spirit of quantum description the temporal evolution spin operator mean value obeys Schrödinger equation

$$
i \hbar \frac{d}{d t}\langle\mathbf{S}\rangle(t)=\langle[\mathbf{S}, \mathbf{H}(t)]\rangle
$$

If the spin is under the sole influence of time dependent external field $(\mathbf{H}(t))$, the Hamiltonian equals Zeeman term (in vacuum)

$$
\mathbf{H}=-\frac{g \mu_{B}}{\hbar} \mathbf{S} \cdot \mathbf{B}, \quad \mathbf{B}=\mu_{0} \mathbf{H}
$$

where $\mu_{\mathrm{B}}$ is the Bohr magneton, $\mathrm{g}$ is the gyromagnetic splitting factor and $\mu_{0}$ the vacuum permeability. After applying usual commutation rules one obtains

$$
\frac{d}{d t}\langle\mathbf{S}\rangle(t)=\frac{g \mu_{B}}{\hbar}(\langle\mathbf{S}\rangle(t) \times \mathbf{B}(t))
$$

It is useful to relate the classical angular momentum of a moving mass to the dipolar moment of a current loop in an uniform magnetic field. The first one reads

$$
\mathbf{L}=m_{e}(\mathbf{r} \times \mathbf{v}),
$$

with $\mathrm{m}_{\mathrm{e}}$ being the electron mass. On the other hand the force exerted on current element (intensity i and length $\mathrm{d} \boldsymbol{l}$ ) by the field $\boldsymbol{B}$ equals

$$
d \mathbf{F}=i d \mathbf{l} \times \mathbf{B} .
$$

After integration the torque exerted by $\boldsymbol{B}$ on the current loop of area A reads

$$
\boldsymbol{\tau}=i \mathbf{A} \times \mathbf{B}, \quad \mathbf{A}=A \mathbf{n},
$$

$\mathbf{n}$ denotes unit vector perpendicular (normal) to loop surface. Analogous to the torque acting on a magnetic dipole due to $\boldsymbol{B}$, the current loop dipole is classically defined as

$$
\mathbf{m}=i \mathbf{A},
$$


where $\mathrm{i}$ - on the case of moving electron reads

$$
i=\frac{q_{e} v}{2 \pi r} .
$$

The charge velocity $v$ and the electron charge qe being negative, and current density being positive lead to the loop dipole moment antiparallel to the angular momentum

$$
\mathbf{m}=\frac{q_{e}}{2 m_{e}} \mathbf{L} .
$$

Similar geometric relation can be written for the spin

$$
\mathbf{m}=\gamma\langle\mathbf{S}\rangle
$$

where $\gamma$ is the gyromagnetic ratio

$$
\gamma=\frac{g q_{e}}{2 m_{e}}=\frac{g \mu_{B}}{\hbar}<0
$$

Now the dynamic Eq. 2.3 can be written in form

$$
\frac{d}{d t} \mathbf{M}(t)=\gamma[\mathbf{M}(t) \times \mathbf{B}(t)]
$$

Defining magnetization as the total dipole per volume $\left(\boldsymbol{M}=\frac{\sum \boldsymbol{m}}{\text { unit vol. }}\right)$ and $\gamma_{0}$ as

$$
\gamma_{0}=\mu_{0} \frac{g\left|\mu_{B}\right|}{\hbar}=-\mu_{0} \gamma
$$

one obtains final form

$$
\frac{d}{d t} \mathbf{M}(t)=-\gamma_{0}[\mathbf{M}(t) \times \mathbf{H}(t)]
$$

Multiplying it successively with $\boldsymbol{M}$ and $\boldsymbol{H}$ leads to

$$
\frac{d}{d t}[\mathbf{M}(t)]^{2}=0,
$$

and

$$
\frac{d}{d t}[\mathbf{M}(t) \cdot \mathbf{H}(t)]=0
$$


Equation 2.15 states that the modulus of the magnetization remains unchanged during the motion and Eq. 2.16 that the angle between the field and the magnetization is constant as function of time. Both equations together provide complete description of magnetization precessional motion around the field with frequency reading

$$
\left|\boldsymbol{\omega}_{0}\right|=\gamma_{0}|\mathbf{H}|
$$

\subsubsection{Damping}

A well known empiric observation is that every system tends to reach an equilibrium state when subject to static field i.e. magnetization precess around the field until it align with it. However, the such static state (i.e. sample magnetization aligned along the field direction) observed experimentally can not be reached as a solution of the precession equations derived in previous paragraph. Therefore, a damping term, making the magnetization align with the field after some finite time, has to be introduced. Standard, phenomenological way to include damping term is to replace the field $\boldsymbol{H}$ in Eq. 2.14 with an effective field including ohmic type dissipation

$$
\mathbf{H}_{e f f}=\mathbf{H}-\alpha \frac{1}{\gamma_{0} M_{s}} \frac{d \mathbf{M}}{d t},
$$

where $M_{\mathrm{s}}$ is the saturation magnetization of the material and $\alpha$ is the damping constant. By inserting 2.18 into 2.14 one obtains the equation known as Landau-Lifshitz equation of magnetization motion

$$
\frac{d}{d t} \mathbf{M}(t)=-\gamma_{0}[\mathbf{M}(t) \times \mathbf{H}(t)]+\frac{\alpha}{M_{s}}\left[\mathbf{M}(t) \times \frac{\mathbf{M}(t)}{d t}\right] .
$$

In order to obtain an explicit expression one has to multiply both sides of Eq. 2.19 by $\boldsymbol{M} \times$ and consider that $\mathbf{M} \times(\mathbf{M} \times \mathbf{M} / d t)=-M_{s}^{2} d \mathbf{M} / d t$. It results in the following dynamic equation, called also Landau-Lifshitz- 
Gilbert equation

$$
\frac{d}{d t} \mathbf{M}(t)=-\frac{\gamma_{0}}{1+\alpha^{2}}[\mathbf{M}(t) \times \mathbf{H}(t)]-\frac{\gamma_{0}}{1+\alpha^{2}} \frac{\alpha}{M_{s}}[\mathbf{M}(t) \times(\mathbf{M}(t) \times \mathbf{H}(t)] .
$$

\subsection{Spin transfer in the diffusive transport limit}

The electron transport model presented in this section is to be used later throughout Chapter 3 and, therefore, here it is explained from the fundamental point of view. In particular, the spin current and spin accumulation are defined and their role in the spin transfer effect is explained. The conditions for diffusive type of transport to be considered are specified and certain constraints of the model that simplify the calculations are presented.

\subsubsection{Spin current and spin accumulation}

In presence of SPC the magnetization is subject to one more torque - ST which is exerted on a ferromagnetic layer due to the absorption of the transverse spin component at the interface. The right side of the corresponding LLG equation is, therefore, supplemented with the new term $\tau$

$$
\frac{d}{d t} \mathbf{M}(t)=-\frac{\gamma_{0}}{1+\alpha^{2}}[\mathbf{M}(t) \times \mathbf{H}(t)]-\frac{\gamma_{0}}{1+\alpha^{2}} \frac{\alpha}{M_{s}}[\mathbf{M}(t) \times(\mathbf{M}(t) \times \mathbf{H}(t)]+\tau
$$

It is the appearance of this term that gives rise to the STT effect which has been lately thoroughly studied both theoretically $[6,7,16]$ and experimentally $[23,11]$. ST treated in the ballistic transport limit was first studied by Slonczewski [6] and Berger [7]. An alternative approach, which is based on theextension of the two-channel model [21], based on solving diffusion equation was provided by Barnas et al. [16].

A typical system where STT can be observed is a trilayer structure sandwiched between semi-infinite electrodes as the one presented in Fig. 2.1. 
The electrodes are typically made of gold or copper. The trilayer consists of the bottom ferromagnetic layer called the fixed (or pinned) layer (PL), the nonmagnetic spacer and ferromagnetic free layer (FL). Often uniform magnetization constant in time is assumed in the PL. Practically it is fabricated thicker than the FL taking the advantage of the fact that ST is only active at the interface. Alternatively, PL magnetization can be fixed by an exchange bias or a synthetic antiferromagnet (SAF) multilayer to fulfill the static assumption.

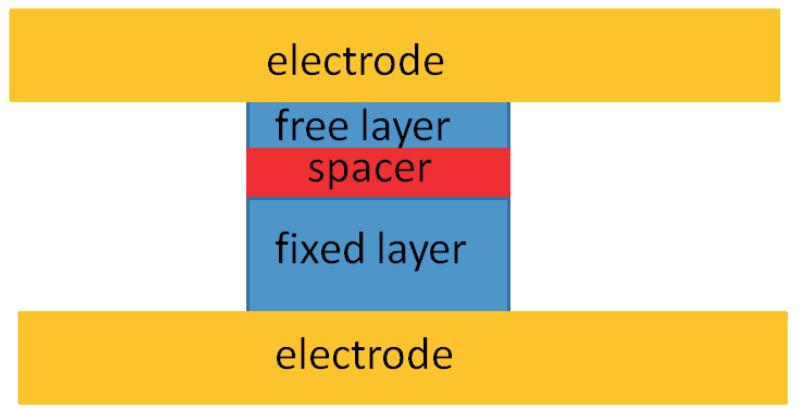

Fig. 2.1: A schematic view of a typical spin valve. The trilayer consists of two ferromagnetic films separated by a nonmagnetic spacer. The whole structure is sandwiched between two electrodes.

In multilayer systems spin dependent mobility, in terms of two-channel model [21], origins from the difference between the electronic bands and scattering cross-section for impurities of majority and minority spins at the Fermi energy. The difference between spin-up and spin-down electric currents defines spin current and the imbalance between the electrochemical potentials determines the spin accumulation which is a non-equilibrium phenomenon. In ferromagnetic films (F) transport properties are determined by free-like s-electrons and magnetic properties by narrow and spin split bands of quasi-localized 3d-electrons which are responsible for rather large spinflip scattering by spin-orbit exchange and at magnetic impurities. Spin-flip scattering destroys the spin accumulation but in CPP geometry it can be 
disregarded provided that the layer thickness is smaller than the spin diffusion length, $\mathrm{l}_{\mathrm{sf}}^{\mathrm{F}}$, which defines the magnetoelectronically active region when in contact with normal metal $(\mathrm{N})$. For the calculation of the spin currents and

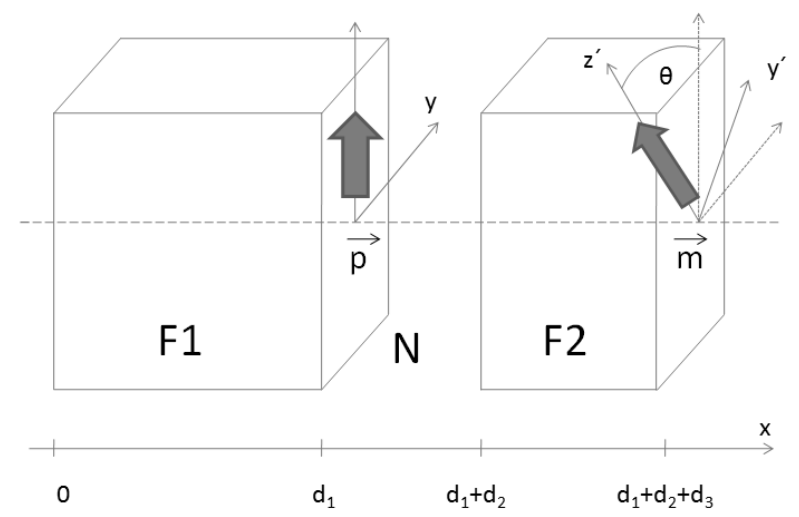

Fig. 2.2: The trilayer under study. F1 and F2 are ferromagnetic film, whereas $\mathrm{N}$ stays for nonmagnetic spacer. The structure is sandwiched between semi-infinite copper electrodes. Because of the noncollinear alignment of the magnetization in F1 and F2 each ferromagnetic layer has its own quantization axis along its magnetization vector direction.

spin accumulation a trilayer consisting of two ferromagnets (F1, F2) separated by a nonmagnetic spacer F1/N/F2 sandwiched between the electrodes has been considered (see Fig. 2.2). The electrodes are assumed to be semiinfinite. A SPC is injected into the nonmagnetic spacer and magnetizes it effectively over a decay length defined by spin diffusion length $\mathrm{l}_{\mathrm{sf}}^{\mathrm{N}}$. The spin relaxation in such nonmagnetic metal can be weak leading to large $\mathrm{l}_{\mathrm{s} f}^{\mathrm{N}}$. The spin accumulation is extended from the interface into the ferromagnet. In a noncollinear system, where the magnetization vectors of F1 and F2 are not parallel, the injected spin current is also noncollinear. As a result, the distribution function inside the film $\check{f}$ is a nondiagonal $2 \times 2$ matrix in the spin space and the spin accumulation can point in any arbitrary direction. Its transverse component cannot penetrate into the F2 and is, therefore, absorbed at the interface transferring angular momentum. This effect is known 
as the spin transfer torque.

In the diffusive transport limit the spatial variation of $\mathrm{f}$ is described by the diffusion equation. Additionally $\mathrm{f}$ is assumed uniform in the film plane and varying only along the axis normal to the film. The way to proceed with the calculations is as follows:

- $2 \times 2$ distribution matrix in spin space for all $\mathrm{F}$ and $\mathrm{N}$ layers in the system is specified

- spin and charge currents though interfaces as function of the distribution matrices in the adjacent film are defined

- matching boundary conditions are introduced

- spin conservation law is applied: difference between total in- and outgoing spin currents equals spin relaxation rate

- continuity of the charge current across all interfaces is observed

- a system of linear equation is solved and all unknown constants are determined by CPP-GMR parameters, and mixing conductance.

The mixing conductance at the interface $\mathrm{N} / \mathrm{F}$ can be interpreted as a measure of the angular momentum transfered from the spin accumulation in the normal metal to the ferromagnetic order parameter. Its imaginary part acts as an effective field. Brief interaction, as the spin current penetrates the ferromagnet (typically couple of Angstroms), makes the spin precess a finite angle around the exchange field. The angular momentum transferred this way affects the ferromagnet in the same way as the magnetic field parallel to the spin accumulation [50]. In presence of conventional scattering processes it governs the STT effect.

Model description is based on [16] and starts with diffusion equation for an arbitrary spin quantization axis [51]

$$
\check{D} \frac{\partial^{2} \check{f}}{\partial x^{2}}=\frac{1}{\tau_{s f}}\left[\check{f}-\check{1} \frac{\operatorname{Tr}\{\check{f}\}}{2}\right]
$$


where $\check{D}$ is the diffusion $2 \times 2$ matrix in the spin space, $\check{1}$ is the $2 \times 2$ unit matrix and $\tau_{s f}$ is the spin-flip relaxation time.

\section{Magnetic film}

In a ferromagnet strong exchange interactions are based on Coulomb interactions and the Pauli principle [50]. Therefore, the component of the distribution function corresponding to the spin orientation normal to local magnetization can be suppressed. If additionally one assumes that the quantization axis is parallel to the local spin polarization, the equations for electrochemical potentials of spin majority $\bar{u}_{\uparrow}$ and spin minority $\bar{u}_{\downarrow}$ electrons can be directly derived from Eq. 2.22 [16]

$$
\begin{aligned}
& \frac{\partial^{2}\left(\bar{u}_{\uparrow}-\bar{u}_{\downarrow}\right)}{\partial x^{2}}=\frac{1}{l_{s f}^{2}}\left(\bar{u}_{\uparrow}-\bar{u}_{\downarrow}\right), \\
& \frac{\partial^{2}\left(\bar{u}_{\uparrow}+\bar{u}_{\downarrow}\right)}{\partial x^{2}}=\eta \frac{\partial^{2}\left(\bar{u}_{\uparrow}-\bar{u}_{\downarrow}\right)}{\partial x^{2}},
\end{aligned}
$$

with spin diffusion length represented as

$$
\frac{1}{l_{s f}^{2}}=\frac{1}{2}\left(\frac{1}{l_{\uparrow}^{2}}+\frac{1}{l_{\downarrow}^{2}}\right),
$$

where $\mathrm{l}_{\uparrow}^{2}=\mathrm{D}_{\uparrow} \tau_{\text {sf }}$ and $l_{\downarrow}^{2}=D_{\downarrow} \tau_{s f}$, and $\eta$ defined as

$$
\eta=-\frac{D_{\uparrow}-D_{\downarrow}}{D_{\uparrow}+D_{\downarrow}} .
$$

Moreover, the electrochemical potential as a sum of an equilibrium contribution $\bar{u}_{0}$ and non-equilibrium part arising from the spin accumulation $\mathrm{g}$ reads

$$
\check{\bar{u}}=\bar{u}_{0} \check{1}+g \bar{\sigma}_{z},
$$

with

$$
\bar{u}_{0}=\frac{1}{2}\left(\bar{u}_{\uparrow}+\bar{u}_{\downarrow}\right)
$$


and

$$
g=\frac{1}{2}\left(\bar{u}_{\uparrow}-\bar{u}_{\downarrow}\right)
$$

Solving Eq. 2.23 and 2.24 provides explicit expressions for $\bar{u}_{0}$ and $\mathrm{g}$

$$
\begin{gathered}
\bar{u}_{0}=\eta\left[\operatorname{Aexp}\left(x / l_{s f}\right)+B \exp \left(-x / l_{s f}\right)\right]+C x+G, \\
g=\operatorname{Aexp}\left(x / l_{s f}\right)+B \exp \left(-x / l_{s f}\right),
\end{gathered}
$$

and the constants A, B, C are to be determined from boundary conditions at proper interfaces. To complete the description of the transport in the ferromagnetic film one has to determine charge and spin currents. For an arbitrary quantization axis the $2 \times 2 \check{\mathrm{j}}$-matrix in the spin space reads

$$
\check{j}=-\check{D} \frac{\partial \check{j}}{\partial x}=-\rho\left(E_{F}\right) \check{D} \frac{\partial \check{\bar{u}}}{\partial x}
$$

where $\rho\left(E_{F}\right)$ is the density of states at the Fermi level per spin. When the quantization axis is parallel to the local spin polarization, $\check{\mathrm{j}}$ might be written as

$$
\check{j}=\frac{1}{2}\left(j_{0} \check{1}+j_{z} \check{\sigma}_{z}\right)
$$

where $j_{0}=\left(j_{\uparrow}+j_{\downarrow}\right)$ is the total particle current and $j_{z}=\left(j_{\uparrow}-j_{\downarrow}\right)$ is the z-component of the spin current. Final expressions read

$$
\frac{1}{\rho\left(E_{F}\right)} j_{0}=-C\left(D_{\uparrow}+D_{\downarrow}\right)
$$

and

$$
\frac{1}{\rho\left(E_{F}\right)} j_{z}=-C\left(D_{\uparrow}-D_{\downarrow}\right)-\frac{2 \tilde{D}}{l_{s f}}\left[A \exp \left(x / l_{s f}\right)-B \exp \left(-x / l_{s f}\right)\right],
$$

where

$$
\tilde{D}=2 \frac{D_{\uparrow} D_{\downarrow}}{D_{\uparrow}-D_{\downarrow}} .
$$

The set of equations $2.30,2.31,2.34,2.35$ provides complete description of the diffusive transport in the ferromagnetic films. 


\section{Nonmagnetic film}

In the $1 \mathrm{D}$ model the spin accumulation becomes a vector in the nonmagnetic film and, thus,

$$
\check{\bar{u}}=\bar{u}_{0} \check{1}+\boldsymbol{g} \cdot \bar{\sigma},
$$

with

$$
\bar{u}_{0}=C x+G,
$$

and

$$
\boldsymbol{g}=\boldsymbol{A} \exp \left(x / l_{s f}\right)+\boldsymbol{B} \exp \left(-x / l_{s f}\right) .
$$

The solutions for spin and charge currents complete the description and read

$$
\begin{gathered}
\check{j}=\frac{1}{2}\left(j_{0} \check{1}+\boldsymbol{j} \check{\boldsymbol{\sigma}}\right) \\
\frac{1}{\rho\left(E_{F}\right)} j_{0}=-2 C D,
\end{gathered}
$$

and

$$
\frac{1}{\rho\left(E_{F}\right)} \boldsymbol{j}=\frac{2 D}{l_{s f}}\left[\boldsymbol{A} \exp \left(x / l_{s f}\right)-\boldsymbol{B} \exp \left(-x / l_{s f}\right)\right] .
$$

Note than all the constants $\boldsymbol{A}, \boldsymbol{B}, \mathrm{C}, \mathrm{G}$ are different in different layers.

\subsubsection{Boundary conditions}

The distribution function and the currents have to be matched at each interface. According to [51] such boundary conditions across normal metal ferromagnet interface in absence of spin-flip scattering at the interface (with the axis $\mathrm{z}$ along the local quantization axis in the ferromagnet) can be written as

$$
\begin{gathered}
e^{2} j_{0}=\left(G_{\uparrow}+G_{\downarrow}\right)\left(\bar{u}_{0}^{F}-\bar{u}_{0}^{N}\right)+\left(G_{\uparrow}-G_{\downarrow}\right)\left(\bar{g}_{0}^{F}-\bar{g}_{0}^{N}\right), \\
e^{2} j_{z}=\left(G_{\uparrow}-G_{\downarrow}\right)\left(\bar{u}_{0}^{F}-\bar{u}_{0}^{N}\right)+\left(G_{\uparrow}+G_{\downarrow}\right)\left(\bar{g}_{0}^{F}-\bar{g}_{0}^{N}\right), \\
e^{2} j_{x}=-2 \operatorname{Re}\left\{G_{\uparrow \downarrow}\right\} g_{x}^{N}+2 \operatorname{Im}\left\{G_{\uparrow \downarrow}\right\} g_{y}^{N}, \\
e^{2} j_{y}=-2 \operatorname{Re}\left\{G_{\uparrow \downarrow}\right\} g_{y}^{N}-2 \operatorname{Im}\left\{G_{\uparrow \downarrow}\right\} g_{x}^{N},
\end{gathered}
$$


where $\boldsymbol{g}^{N}\left(g^{F}\right)$ is the spin accumulation on the N (F) side of the interface, $G_{\uparrow}$ and $G_{\downarrow}$ are the interfacial conductances in the spin majority and spin minority channels and $G_{\uparrow \downarrow}$ is the spin mixing conductance of the interface.

This set of boundary conditions has to obeyed at all interfaces. One should also observe physical constraints for potential in the semi-infinite electrodes and continuity of the particle current across all interfaces. Moreover, distribution functions and spin currents in magnetic films are written in the coordinate system whose axis is along the local spin polarization. Therefore, in the nonmagnetic film these equations are written in the system whose $\mathrm{z}$-axis coincides with the local quantization axis of one of the adjacent ferromagnetic films. Each ferromagnet has different quantization axis and, hence, the distribution function and the spin current have to be transformed from one system to the other. All above mentioned equations hold but additionally spin current and spin accumulation in the nonmagnetic spacer shall be first transformed

$$
\begin{gathered}
g_{x}^{\prime}=g_{x} \sin \phi-g_{y} \cos \phi, \\
g_{y}^{\prime}=g_{x} \cos \phi \cos \theta+g_{y} \sin \phi \sin \theta-g_{z} \sin \theta, \\
g_{z}^{\prime}=g_{x} \cos \phi \sin \theta+g_{y} \sin \phi \sin \theta+g_{z} \cos \theta
\end{gathered}
$$

The spin accumulation (from $\mathrm{N}$ ) with polarization normal to the magnetization direction cannot penetrate the ferromagnet (F2). Therefore, the transverse component of the spin current at the interface is absorbed exerting torque on F2, which can be written as the difference of the normal components of the spin currents at the left and right interface of the nonmagnetic spacer

$$
\boldsymbol{\tau}=\frac{\hbar}{2}\left(\boldsymbol{j}_{\perp L}-\boldsymbol{j}_{\perp R}\right)
$$

\subsubsection{Spin torque}

This section presents thoroughly torque calculations and link them with the CPP-GMR experimental data. For the numeric implementation of the torque 
in the diffusive limit it is convenient to divide it into

$$
\tau_{\theta}=a I \mathbf{m} \times(\mathbf{m} \times \mathbf{p}),
$$

and

$$
\tau_{\phi}=b I \mathbf{m} \times \mathbf{p},
$$

where $\boldsymbol{m}$ and $\boldsymbol{p}$ are unit vectors along the magnetization of the F1 and F2 and $\mathrm{I}$ is the charge current. Alternatively one can write them in a scalar form

$$
\tau_{\theta}=a I \sin \theta
$$

and

$$
\tau_{\phi}=-b I \sin \theta
$$

On the other hand Eq. 2.50 yields

$$
\tau_{\theta}=-\left.\frac{\hbar}{2} j_{y}^{\prime}\right|_{x \rightarrow x_{0}}=-\left.\frac{\hbar}{2}\left(j_{x} \cos \phi \cos \theta+j_{y} \sin \phi \sin \theta\right)\right|_{x \rightarrow x_{0}},
$$

and

$$
\tau_{\phi}=\left.\frac{\hbar}{2} j_{x}^{\prime}\right|_{x \rightarrow x_{0}}=\left.\frac{\hbar}{2}\left(j_{x} \sin \phi-j_{y} \cos \theta\right)\right|_{x \rightarrow x_{0}},
$$

where $\mathrm{x}_{0}=\mathrm{d}_{1}+\mathrm{d}_{2}$ denotes the position of the active interface in the spin valve. Comparing Eq. 2.53 with Eq. 2.55 and Eq. 2.54 with Eq. 2.56 one obtains parameters $a$ and $b$ as functions of spin currents. Having solved the boundary condition for the spin currents and inserting the solution into Eq. 2.55 and Eq. 2.56 yields $a$ and $b$ as functions of sole parameter $\theta$. Numerically they are to be calculated as follows. A solver for system of linear equations provided in Mathematica [48] has been employed for development of a code aimed at the determination of all constant parameters entering boundary conditions.

At this point one should note the correlation between STT and CPPGMR experimental data as these serve as input data in the developed application. The GMR is related to the spin dependent electronic conduction in ferromagnetic metals and the model description presented in this chapter 
accounts for this data as follows. The asymmetry of spin majority and spin minority channels can be described by standard definitions [21]

$$
\rho_{\uparrow(\downarrow)}=2 \rho^{*}(1 \pm \beta),
$$

and

$$
R_{\uparrow(\downarrow)}=2 R^{*}(1 \mp \gamma),
$$

where $\rho_{\uparrow}, \rho_{\downarrow}$ are the bulk resistivities, $R_{\uparrow}, R_{\downarrow}$ interface resistances per unit square for spin majority and spin minority electrons respectively, $\beta$ and $\gamma$ are the bulk and interfacial asymmetry coefficients. For nonmagnetic layers Eq. 2.57 is used with $\beta=0$. The conductances $G_{\uparrow}, G_{\downarrow}$ are equal to $G_{\uparrow}=1 / R_{\uparrow}$ and $G_{\downarrow}=1 / R_{\downarrow}$. The bulk parameters entering the description i.e. mean free paths and diffusion constants are expressed in frames of a free electron model by using parameters defined in Eq. 2.57 and relevant Fermi energy, which is assumed to be equal in magnetic and nonmagnetic layer. The diffusion parameter can be then expressed as

$$
D_{\uparrow(\downarrow)}=\frac{1}{3} v_{F} \lambda_{\uparrow(\downarrow)},
$$

where $v_{F}=\sqrt{2 E_{F} / m_{e}}$ is the Fermi velocity of electrons and the mean free paths $\lambda_{\uparrow(\downarrow)}$ are:

$$
\lambda_{\uparrow(\downarrow)}=\frac{m_{e} v_{F}}{n e^{2} \rho_{\uparrow(\downarrow)}},
$$

with $m_{e}$ electron mass and $n=\left(1 / 6 \pi^{2}\right)\left(2 m_{e} E_{F} / \hbar^{2}\right)^{3 / 2}$ being the density of electrons per spin. Moreover $\rho\left(E_{F}\right)$ is given by $\rho\left(E_{F}\right)=\left(1 / 4 \pi^{2}\right)\left(2 m_{e} / \hbar^{2}\right)^{3 / 2} E_{F}^{1 / 2}$. Furthermore assumption of the free electron model leads to $\frac{\lambda_{\uparrow}}{\lambda_{\downarrow}}=\frac{1-\beta}{1+\beta}$ and $\eta$ is determined by simple relation

$$
\eta=-\beta
$$

For nonmagnetic layers the same definitions hold but $\lambda_{\uparrow(\downarrow)}$ and $D_{\uparrow(\downarrow)}$ are independent of the spin orientation. Spin diffusion lengths enter description as 
independent parameters and values shall be taken from GMR experiments. Two remaining parameters: real and imaginary part of the mixing conductance are to be derived from ab inito calculations [52]. Combining all above mentioned relations the torque has been calculated from Eq. 2.50 and eventually $a$ and $b$ (scaled with $\hbar /|e|$ ) have been fitted with a series of cosine functions

$$
\begin{aligned}
& a(\theta)=a_{0}+a_{1} \cos (\theta)+a_{2} \cos ^{2}(\theta)+a_{3} \cos ^{3}(\theta)+\ldots, \\
& b(\theta)=b_{0}+b_{1} \cos (\theta)+b_{2} \cos ^{2}(\theta)+b_{3} \cos ^{3}(\theta)+\ldots .
\end{aligned}
$$

One can compare angular variation of the ST for a symmetric (Fig. 2.3a) and an asymmetric (Fig. 2.3b) pillar structure. The term symmetric refers to a pillar where both ferromagnetic layers are made of the same material, whereas in the asymmetric one two different materials are used. The first one resembles the standard torque [6] and supports standard behavior i.e. in absence of external field current-induced switching can be observed whereas in the presence of applied fields higher than the coercive field, the generation of microwave oscillations is possible. The uniqueness of the ST from Fig. 2.3b manifests itself very clearly. It is the existence of certain critical angle $\theta$ at which the torque vanishes, that induces interesting dynamics at zero and low field. Above the threshold current both collinear states of the magnetization are unstable and the only solution of the LLG equation is the steady state precession or a noncollinear static magnetization state $[53,16]$. It is worth emphasizing that the out-of-plane (OP) torque is 2 orders of magnitude smaller than its in-plane (IP) component, which means that the latter does not markedly influence the magnetization dynamics.

\subsection{Mesoscale models}

Within this work two models to describe magnetization dynamics are employed. In the limiting assumption of a quasi-uniform magnetization within 


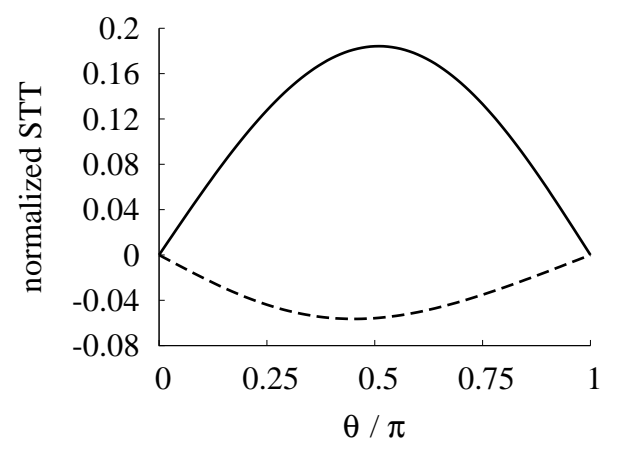

(a)

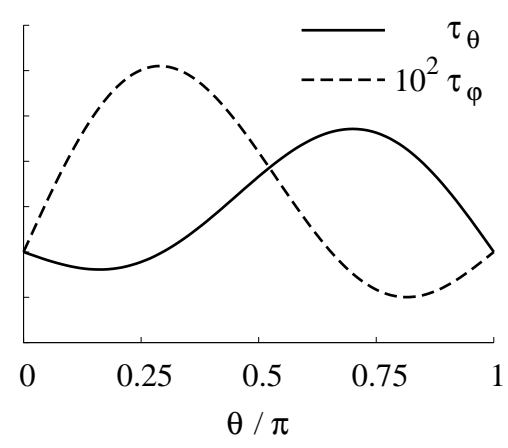

(b)

Fig. 2.3: Angular dependence of the spin transfer torque normalized to $\hbar j /|e|$ for (a) symmetric $\mathrm{Py}(8 \mathrm{~nm}) / \mathrm{Cu}(10 \mathrm{~nm}) / \mathrm{Py}(8 \mathrm{~nm})$ and (b) asymmetric $\mathrm{Co}(8 \mathrm{~nm}) / \mathrm{Cu}(10 \mathrm{~nm}) / \mathrm{Py}(8 \mathrm{~nm})$ pillar.

the particle the macrospin approach can be used. However, real systems may undergo complicated transitions, which are far beyond this simple approach. Therefore, thorough understanding of physics behind observed phenomena calls for more sophisticated model. Micromagnetic approach, assuming magnetization as a continuous function of position, provides a powerful tool for study of magnetization dynamics.

\subsubsection{Macrospin model}

Macrospin model, often referred to as single domain model, treats the sample as a single spin in the whole volume. The energy functional of such element can be written as

$$
\epsilon=\epsilon_{K}-\frac{1}{2} \mu_{0} M_{s}^{2} \mathbf{m} \cdot \mathbf{h}_{d}-\mu_{0} M_{s}^{2} \mathbf{m} \cdot \mathbf{h}_{\text {ext }},
$$

where $\hat{x}$ defines the easy axis, $\mathrm{K}$ refers to the anisotropy, $\mathbf{h}_{d}$ the demagnetizing field and $\mathbf{h}_{\text {ext }}$ the external field. The normalized magnetization vector $\boldsymbol{m}$ reads

$$
\mathbf{m}=\frac{\mathbf{M}}{M_{s}}
$$


The first term in 2.64 is the uniaxial anisotropy, the second term describes demagnetizing effects (self magnetostatic term) and the third term the Zeeman energy. The effective field reads

$$
\mathbf{h}_{e f f}=-\frac{1}{\mu M_{s}^{2}} \frac{\delta \epsilon}{\delta \mathbf{m}}=\mathbf{h}_{K}+\mathbf{h}_{d}+\mathbf{h}_{e x t}
$$

and is introduced into Eq. 2.20 as the field term. The obtained dynamic equation is the first order ordinary differential equation (ODE) for a single spin.

\subsubsection{Micromagnetic model}

Micromagnetics, treating the magnetization as continuum, provides the possibility of dealing with spatially nonuniform states. In general the density functional can be written as

$$
\epsilon=A(\nabla \mathbf{m})^{2}+\epsilon_{k}-\frac{1}{2} \mu_{0} M_{s}^{2}\left(\mathbf{m} \cdot \mathbf{h}_{d}\right)-\mu_{0} M_{s}^{2}\left(\mathbf{m} \cdot \mathbf{h}_{e x t}\right) .
$$

The exchange energy

First of all, the finite exchange energy is introduced. Hund's rules determines energy minimization by ordering electrons into nearly degenerate atomic levels. The first rule imposes spin maximization i.e. as many electrons with spin in one direction are gathered in partially filled atomic orbital before spins in other direction are added. The energy gain that motivates Hund's rule is that Pauli exclusion keeps the electrons with the same spin further apart on average, thereby lowering the Coulomb repulsion between them. This is called the exchange energy. The transition metal ferromagnets exhibit both: strong exchange splitting and strong hybridization. The exchange splitting stabilizes a spin-polarized ferromagnetic state by generating a self-consistent shift of the majority-electron band states to lower energy than the minorityelectron spin states. That way, the kinetic energy cost associated with polarization formation is compensated [54]. The micromagnetic exchange is 
the interaction that tends to keep all magnetization moments aligned in a common direction adding an energy cost when the magnetization rotates as function of position. From quantum point of view, the exchange hamiltonian reads

$$
W_{i, j}=-2 J \mathbf{S}_{i} \cdot \mathbf{S}_{j}
$$

where $\mathrm{J}$ is a constant and $\mathbf{S}_{i}$ is the angular momentum of the $\mathrm{i}-$ th spin. It describes just the first neighbor interaction since $\mathrm{J}$ decreases rapidly. If the angle between neighboring spins $\theta_{i j}$ is considered to be small it can be written

$$
\mathbf{S}_{i} \cdot \mathbf{S}_{j}=S^{2}\left(1-\frac{1}{2} \theta_{i j}^{2}\right)=S^{2}\left[1-\frac{1}{2}\left|\mathbf{m}_{j}-\mathbf{m}_{i}\right|^{2}\right],
$$

where $S=\left|\mathbf{S}_{i}\right|=\left|\mathbf{S}_{j}\right|$ and $\mathbf{m}_{i}$ is the unity magnetization vector (antiparallel to the angular momentum). If one assumes $\mathbf{m}=\alpha \mathbf{i}+\beta \mathbf{j}+\gamma \mathbf{k}$ the position can be approximated with a continuous function

$$
\alpha_{i}-\alpha_{j}=\mathbf{v}_{j} \cdot \Delta \alpha, \beta_{i}-\beta_{j}=\mathbf{v}_{j} \cdot \Delta \beta, \gamma_{i}-\gamma_{j}=\mathbf{v}_{j} \cdot \Delta \gamma,
$$

with $\mathbf{v}_{j}$ denoting position the the $\mathrm{j}$-spin with respect to i-spin. Subtracting the constant term, which has no influence on dynamics, and combining 2.69 with 2.70 one can write Eq. 2.68 in form

$$
W_{i j}=J S^{2}\left|\mathbf{m}_{j}-\mathbf{m}_{i}\right|^{2}=J S^{2}\left[\left(\mathbf{v}_{j} \Delta \alpha\right)^{2}+\left(\mathbf{v}_{j} \Delta \beta\right)^{2}+\left(\mathbf{v}_{j} \Delta \gamma\right)^{2}\right] .
$$

Finally, the exchange energy density per volume in a sample with $\mathrm{n}$ spins reads

$$
\epsilon_{e x}=\frac{1}{2} n J S^{2} \sum_{j}\left[\left(\mathbf{v}_{j} \Delta \alpha\right)^{2}+\left(\mathbf{v}_{j} \Delta \beta\right)^{2}+\left(\mathbf{v}_{j} \Delta \gamma\right)^{2}\right] .
$$

Note that only first neighbors are considered and factor $1 / 2$ is introduced to avoid double count of interaction in each spin pair. For a simple cubic lattice with the atomic length $a$ and where $\left|\mathbf{v}_{j}\right|=a$ and $n=a^{-3}$ the exchange energy density is reduced to

$$
\epsilon_{e x}=A\left[(\nabla \alpha)^{2}+(\nabla \beta)^{2}+(\nabla \gamma)^{2}\right]
$$


where $\mathrm{A}$ is called the exchange constant and reads

$$
A=\frac{J S^{2}}{a} \text {. }
$$

Equation 2.73 was derived for a simple cubic lattice but in general it holds also for different types of lattices. The exchange constant $A$ strongly depending on the temperature introduces temperature dependence into 2.73. In the continuous approximation one can write hamiltonian in form

$$
E_{\text {exch }}=\int_{V} \epsilon_{\text {ex }} d V=\int_{V} A(\nabla \mathbf{m})^{2} d V
$$

which leads directly to the expression for the exchange field

$$
\mathbf{h}_{\text {exch }}=\frac{2 A}{\mu_{0} M_{S}^{2}}(\nabla \mathbf{m})^{2} .
$$

The anisotropy energy

The magnetocrystalline anisotropy defines preferential magnetization orientation in a material and depends on the crystalline structure of the material. The associated energy represents the energy cost of magnetizing the sample in certain direction with respect to the favored direction. The energy functional for the anisotropy contribution is given for uniaxial and cubic materials as

$$
\epsilon_{K, u}=K_{1}(\mathbf{m} \cdot \mathbf{u})^{2}
$$

where $\mathbf{u}$ denotes the easy axis and

$$
\epsilon_{K, c}=K_{1}\left(m_{x}^{2} m_{y}^{2}+m_{y}^{2} m_{z}^{2}+m_{x}^{2} m_{z}^{2}\right)+K_{2}\left(m_{x}^{2} m_{y}^{2} m_{z}^{2}\right),
$$

respectively. The associated field expressions read

$$
\begin{gathered}
\mathbf{h}_{K, u}=\frac{2 K_{1}}{\mu_{0} M_{S}^{2}}\left(\mathbf{m} \cdot \mathbf{u}_{K}\right) \mathbf{u}_{K}, \\
\mathbf{h}_{K, c}=-\frac{2 D}{\mu_{0} M_{S}^{2}} \mathbf{m}
\end{gathered}
$$

where $\mathrm{D}$ is a diagonal matrix [55]. 
The self magnetostatic energy

The energy associated with the magnetostatic interaction in the sample is called self magnetostatic or demagnetizing energy. Even though the system is discrete one can apply continuous description of this interaction not forgetting about its discrete character

$$
\epsilon_{d}=-\frac{1}{2} \mu_{0} M_{s}^{2} \mathbf{m} \cdot \mathbf{h}_{d}
$$

with $\boldsymbol{h}_{d}$ being self magnetostatic field that reads

$$
\mathbf{h}_{d}(\mathbf{r})=\frac{1}{4 \pi M_{s}} \int_{V} \frac{\rho_{M}\left(\mathbf{r}^{\prime}\right) \mathbf{R}}{R^{3}} d V^{\prime}+\frac{1}{4 \pi M_{s}} \int_{S} \frac{\sigma_{M}\left(\mathbf{r}^{\prime}\right) \mathbf{R}}{R^{3}} d S^{\prime},
$$

where $\boldsymbol{R}=\boldsymbol{r}-\boldsymbol{r}^{\prime}$ and $R=|\boldsymbol{R}|=\left|\boldsymbol{r}-\boldsymbol{r}^{\prime}\right|$, V denotes volume, S surface. This field, analogous to the electric field created by the electric charges, is calculated by integrating over the volume and surface charges, which are defined as follows

$$
\begin{gathered}
\rho_{M}(\mathbf{r})=-\nabla \mathbf{M}(\mathbf{r}), \\
\sigma_{M}(\mathbf{r})=\mathbf{n} \cdot \mathbf{M}(\mathbf{r}),
\end{gathered}
$$

where $\mathbf{n}$ denotes unit vector normal to the surface S.

The energy density functional based solely on exchange and magnetostatic terms leads to definition of a micromagnetically important factor called the exchange length

$$
\lambda=\sqrt{\frac{2 A}{\mu_{0} M_{s}^{2}}} .
$$

This is material characteristic parameter (temperature dependent) and in ferromagnetic materials and their alloys rarely exceeds few nanometers imposing severe constraints on the mesh size in numerical simulations [55]. 


\section{External field}

The energy density associated with the external field in continuous approximation reads

$$
W_{e x t}=-\mu_{0} M_{s} \int_{V} \mathbf{h}_{e x t}(\mathbf{r}) \cdot \mathbf{m}(\mathbf{r}) d V,
$$

which leads to the field contribution

$$
\frac{\epsilon_{e x t}}{\mu_{0} M_{s}}=-\mathbf{m} \cdot \mathbf{h}_{e x t}
$$

\subsection{Computational techniques}

For the macrospin study presented in Chapter 3 the self magnetostatic contribution to the effective field in macrospin approximation is calculated based on the demagnetizing tensor coefficients given in [56]. For the time integration of Eq. 2.21, Heun solver was employed [57]. Stability studies were carried out in order to assign proper time integration step.

In the micromagnetic model magnetization and external field are assumed continuous and, therefore, 2.20 is an differential equation for which analytical solutions do not exist except for a very few idealized case. For problems of practical interest it needs to be solved numerically. In order to do so a spatial discretization of the sample volume and, thus, all expressions for energy and field terms derived in the continuous limit have to translated to be consistent with the discrete description. The discretization of the continuous magnetization vectorial field is done as follows $\boldsymbol{M}(i, j, k)=M_{s} \boldsymbol{m}(i, j, k)$, where $i: 1, \ldots, N_{x}, j: 1, \ldots, N_{y}, k: 1, \ldots, N_{z}$, with $N_{x}, N_{y}, N_{z}$ being the number of cells in each cartesian direction. This way the volume of each cell reads $\Delta V=\Delta x \Delta y \Delta z$ with modulus of $\mathrm{M}_{\mathrm{s}}$ being constant inside and the magnetization in each cell is assumed to be uniform. The magnetization is treated as a continuous function of the position in the material. Once the system is discretized a set of ODEs is obtained, which are solved using fourth order Runge-Kutta solver. 
Moreover the cell size has to be chosen smaller than the exchange length in order to resolve the spatial variations of magnetization correctly. Thus, the size of the computational cell has to observe these constraints.

\subsubsection{Exchange energy}

The discrete form of the exchange energy density reads

$$
\epsilon_{e x}(i, j, k)=A\left[\left(\nabla m_{x}(i, j, k)\right)^{2}+\left(\nabla m_{y}(i, j, k)\right)^{2}+\left(\nabla m_{z}(i, j, k)\right)^{2}\right]
$$

where following notation is used for each cartesian component $\alpha=x, y, z$,

$$
(\nabla m)^{2} \equiv\left(\frac{\partial m_{\alpha}}{\partial x}\right)^{2}+\left(\frac{\partial m_{\alpha}}{\partial y}\right)^{2}+\left(\frac{\partial m_{\alpha}}{\partial z}\right)^{2} .
$$

In the finite difference approximation the derivatives are substituted with the ratios of value increments in the nodes of the mesh or in the centers of each computational cell as follows

$$
(\nabla m)^{2} \approx\left(\frac{\Delta_{x} m_{\alpha}}{\Delta x}\right)^{2}+\left(\frac{\Delta_{y} m_{\alpha}}{\Delta y}\right)^{2}+\left(\frac{\Delta_{z} m_{\alpha}}{\Delta z}\right)^{2},
$$

where $\Delta x, \Delta y, \Delta z$ define the size, whereas $\Delta_{x}, \Delta_{y}, \Delta_{z}$ represent finite difference operators in each direction. In each point the magnetization reads $\boldsymbol{M}(i, j, k)=M_{s} \boldsymbol{m}(i, j, k)$ and, therefore, in the neighboring cell (in $+\mathrm{x}$ direction) the expression takes form $\boldsymbol{M}(i+1, j, k)=M_{s} \boldsymbol{m}(i+1, j, k)$. Thus, we can write the first term of 2.90 as

$$
\left(\frac{\Delta_{x} m_{\alpha}}{\Delta x}\right)^{2}=\left(\frac{m_{x}(i+1, j, k)-m_{x}(i, j, k)}{\Delta x}\right)^{2} .
$$

Considering that for each cell $|\boldsymbol{m}(i, j, k)|=1$ holds, Eq. 2.91 can be simplified

$$
\left(\frac{\Delta_{x} m_{\alpha}}{\Delta x}\right)^{2}=\frac{1}{(\Delta x)^{2}}\left[2-2 m_{x}(i, j, k) m_{x}(i+1, j, k)\right] .
$$


One derives analogous formulas for y and z-component and additionally considers the next neighbors situated in the negative directions, obtaining finally

$$
\begin{array}{r}
\epsilon_{e x}(i, j, k)=\frac{2 A}{(\Delta x)^{2}} \sum_{i^{\prime}, j^{\prime}, k^{\prime}}^{N N}\left[1-\mathbf{m}(i, j, k) \cdot \mathbf{m}\left(i^{\prime}, j^{\prime}, k^{\prime}\right)\right]= \\
N N \frac{2 A}{(\Delta x)^{2}}-\frac{2 A}{(\Delta x)^{2}} \mathbf{m}(i, j, k) \cdot \sum_{i^{\prime}, j^{\prime}, k^{\prime}}^{N N} \mathbf{m}\left(i^{\prime}, j^{\prime}, k^{\prime}\right) .
\end{array}
$$

The exchange energy in the numeric form is expressed as

$$
W_{e x}=\int_{V^{\prime}} \epsilon_{e x}\left(\mathbf{r}^{\prime}\right) d V^{\prime} \approx \sum_{(i, j, k)}^{N_{c}} \epsilon_{e x}(i, j, k) \Delta V,
$$

and the associated field term reads

$$
\begin{array}{r}
\mathbf{h}_{e x}(i, j, k)=\frac{2 A}{\mu_{0} M_{s}}\left[\frac{\mathbf{m}(i+1, j, k)+\mathbf{m}(i-1, j, k)}{(\Delta x)^{2}}+\right. \\
\left.\frac{\mathbf{m}(i, j+1, k)+\mathbf{m}(i, j-1, k)}{(\Delta y)^{2}}+\frac{\mathbf{m}(i, j, k+1)+\mathbf{m}(i, j, k-1)}{(\Delta z)^{2}}\right] .
\end{array}
$$

The approximation of the partial derivatives with the finite differences only holds provided that the angle between the magnetization vectors of the neighboring cells is small.

\subsubsection{Anisotropy energy}

The anisotropy, representing local interaction, can be easily translated into a form compatible with the micromagnetic assumptions. The numeric expressions for the uniaxial anisotropy energy, the energy density and the field simply read

$$
\begin{gathered}
W_{u}=\sum_{(i, j, k)}^{N_{c}} \epsilon_{u}(i, j, k) \Delta V, \\
\epsilon_{u}(i, j, k)=K\left[1-\left(\mathbf{m}(i, j, k) \cdot \mathbf{u}_{K}\right)^{2}\right], \\
\mathbf{h}_{u}(i, j, k)=\frac{2 K}{\mu_{0} M_{s}}\left(\mathbf{m}(i, j, k) \cdot \mathbf{u}_{K}\right) \mathbf{u}_{K},
\end{gathered}
$$

with $\boldsymbol{u}_{\boldsymbol{K}}$ denoting unit vector of the uniaxial anisotropy direction. 


\subsubsection{Self magnetostatic energy}

The conversion of the self magnetostatic term into a numerically compatible form, owing its nonlocal character, is more sophisticated. The contribution of the demagnetizing field $\boldsymbol{h}_{d}(\boldsymbol{r})$ in each cell depends on the magentization $\boldsymbol{m}\left(\boldsymbol{r}^{\prime}\right)$ in all other nodes and reads

$$
\mathbf{h}_{d}(\mathbf{r})=\sum_{\alpha} h_{d}^{\alpha} \mathbf{u}_{\alpha}
$$

where $\alpha:(x, y, z), h_{d}^{\alpha}$ denotes $\alpha$-component of the demagnetizing dipolar field and $\boldsymbol{u}_{\alpha}$ is the unit vector of each cartesian direction. Thus, one can write

$$
h_{d}^{\alpha}(i, j, k)=\sum_{\beta}^{(x, y, z)} \sum_{\left(i^{\prime}, j^{\prime}, k^{\prime}\right)}^{N_{c}} N_{\alpha \beta}\left(i-i^{\prime}, j-j^{\prime}, k-k^{\prime}\right) m_{\beta}\left(i^{\prime}, j^{\prime}, k^{\prime}\right),
$$

with factors $N_{\alpha \beta}\left(i-i^{\prime}, j-j^{\prime}, k-k^{\prime}\right)$ representing the components of the demagnetizing tensor. Since they depend only on the geometry of the system, they are calculated just once and used thereafter for the rest of the simulation.

Within this work the self magnetostatic field term is calculated for 3D discretization using the demagnetizing tensor expressions from Ref. [58] under assumption that the magnetization is constant within the volume of each cell. Numeric calculation of the demagnetizing field is very time consuming due to the summing over all elements of the mesh. Therefore, to reduce the computational time Fast Fourier Transform (FFT) is introduced.

In the cartesian space each component of the demagnetizing field is a convolution and thus in the phase space such $3 \mathrm{D}$ convolution can be translated in a simple scalar product

$$
\begin{aligned}
& \tilde{h_{d}^{\alpha}}\left(k_{x}, k_{y}, k_{z}\right) \equiv \mathcal{F}\left[h_{d}^{\alpha}(i, j, k)\right]=\sum_{\beta}^{(x, y, z)} \mathcal{F}\left[N_{\alpha \beta}(i, j, k)\right]\left[m_{\beta}(i, j, k)\right]= \\
& \sum_{\beta}^{(x, y, z)}\left[\tilde{N}_{\alpha \beta}\left(k_{x}, k_{y}, k_{z}\right)\right]\left[\tilde{m}_{\beta}\left(k_{x}, k_{y}, k_{z}\right)\right] .
\end{aligned}
$$


The calculation is carried out as follows. The demagnetizing coefficients of the tensor $N_{\alpha \beta}$ are evaluated and its Fourier transform is obtained. Also the Fourier Transform of the magnetization in each cell is determined. The final expression for the demagnetizing field in the cartesian space requires an inverse Fourier Transform to be applied. Clearly the FFT is limited to a translational invariant system and additionally zero-padding technique [57] is used in order to overcome this limitation. The energy density and the demagnetizing field in their numeric form read

$$
\begin{gathered}
\epsilon_{d}(i, j, k)=-\frac{1}{2} \mu_{0} M_{s}^{2} \sum_{\alpha} h_{d}^{\alpha}(i, j, k) m_{\alpha}(i, j, k), \\
h_{d}^{\alpha}(i, j, k) \equiv \mathcal{F}^{-1}\left[\tilde{h_{d}^{\alpha}}\left(k_{x}, k_{y}, k_{z}\right)\right]=\mathcal{F}^{-1}\left[\sum_{\beta}^{(x, y, z)} \mathcal{F}\left[N_{\alpha \beta}(i, j, k)\right] \mathcal{F}\left[m_{\beta}(i, j, k)\right]\right] .
\end{gathered}
$$

\subsubsection{External field energy}

Since the interaction with the external field is a local interaction, the discretization of the expression for the energy density is trivial

$$
\epsilon_{e x t}(\mathbf{r})=-\mu_{0} M_{s}^{2} \mathbf{m}(\mathbf{r}) \cdot \mathbf{h}_{\text {ext }}(\mathbf{r})
$$

where $\boldsymbol{h}_{\text {ext }}(\boldsymbol{r})$ is the normalized external field (in units of $\mathrm{M}_{\mathrm{s}}$ ). 


\section{SPIN TORQUE DRIVEN OSCILLATIONS IN AN ASYMMETRIC SPIN VALVE}

In this section a systematic study of current-induced oscillations in a nanopillar of composition $\mathrm{Co}(8 \mathrm{~nm}) / \mathrm{Cu}(10 \mathrm{~nm}) / \mathrm{Py}(8 \mathrm{~nm})$ and an elliptical crosssection $155 \times 100 \mathrm{~nm}^{2}$ is presented. The ST is obtained from the diffusive transport theory as derived in Chapter 2. The motivation for this study is following: this system has been recently studied experimentally $[17,18]$ and shows some interesting properties such as oscillations in absence of external field. Interestingly, reported behavior can not be explained within the standard Slonczewki ST model. Thus, implementation of the diffusive limit ST already in a simple macrospin model leads to some explanations of reported trends. Still, some aspects like nonlinear frequency blueshift with current and saturation regime are not reproduced within macrospin study. Therefore, both macrospin and full micromagnetic simulations are performed to characterize the dynamic response of the system.

\subsection{Specifications}

Quasi uniform magnetization precession in the FL leads to the change of the output GMR-signal, whose variations are translated into the output measured power. Therefore, the latter depends indirectly on the oscillation axis direction which defines the angle between the magnetization direction in the FL and the PL. Figure 3.1 shows a schematic representation of the system under study. In the discussion that follows the direction of the oscillation axis 
distinguishes between the out-of-plane precession (OPP) and the in-plane precession (IPP) in the free layer. In the first case, as shown in red, the symmetry axis is close to the OP direction whereas in the the latter somewhere in the xy-plane. Hence, the OPP is of particular interest as it provides higher output power as compared to the IPP. Additionally, depending on the orientation of the axis one specifies either $\mathrm{IPP}_{+}\left(\mathrm{OPP}_{+}\right)$or IPP $\left.\mathrm{IPPP}_{-}\right)$.

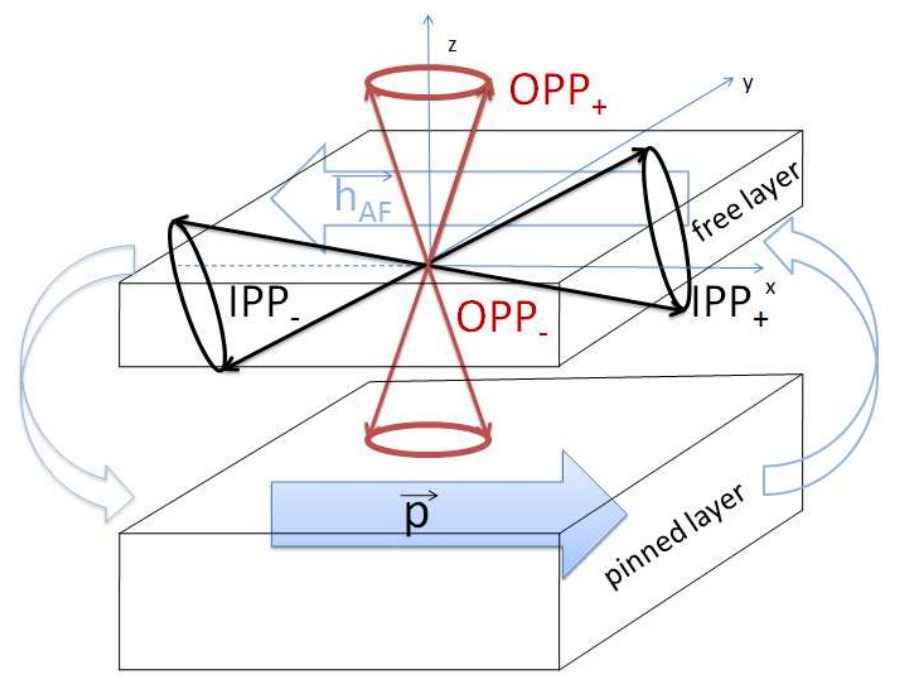

Fig. 3.1: The direction of the oscillation axis defines the out-of-plane (red) and the in-plane precession (black). Its orientation specifies furter positive and negative index of the mode.

In this section first the effect of deviations in the wavy torque shape on the dynamics is shown. Later, different oscillatory modes supported by combined influence of the wavy torque and the external field are identified. Finally, analysis of the dynamics supported in absence of external field is carried out. It is shown that experimentally observed trends, which are beyond macrospin description, have been successfully predicted within micromagnetic framework.

Two different geometries are investigated. First an extended structure, where due to large lateral size of the pinned layer, its magnetostatic contri- 
bution, called throughout this chapter interlayer coupling field (ICF), to the effective field acting on the free layer can be neglected. In this case, the main contribution to the effective field is the self magnetostatic field term. Second, an etched pillar has been considered, where the etched pinned layer gives rise to the ICF, significantly changing the effective field in the FL. The ICF is the magnetostatic field exerted in the FL originating from the existence of the ferromagnetic PL as shown in Fig. 3.1.

Only the dynamics of the FL is resolved assuming PL to stay uniformly magnetized in time. The final computational form of the dynamic Eq. 2.19 including torque and introducing dimensionless variables $\mathrm{m}=\mathrm{M} / \mathrm{M}_{\mathrm{s}}$ and $\mathrm{h}=\mathrm{H} / \mathrm{M}_{\mathrm{s}}$ reads

$$
\frac{1+\alpha^{2}}{\gamma_{0} M_{\mathrm{S}}} \frac{d \mathbf{m}}{d t}=-\mathbf{m} \times \mathbf{h}_{\mathrm{eff}}-\alpha \mathbf{m} \times\left(\mathbf{m} \times \mathbf{h}_{\mathrm{eff}}\right)+\frac{\boldsymbol{\tau}}{\mu_{0} M_{\mathrm{S}}^{2} L_{z}}
$$

where $\mathbf{m}$ is the magnetization vector of the $\mathrm{FL}, \mathbf{p}$ is the magnetization vector of the $\mathrm{PL}, \mathrm{h}_{\mathrm{eff}}$ is the effective field and $\mathrm{M}_{\mathrm{s}}$ is the saturation magnetization of the free layer and $\mathrm{L}_{\mathrm{z}}$ is its thickness. The last term in Eq. 3.1 corresponds to the prefactor and ST itself $(\boldsymbol{\tau})$. ST is calculated from Eq. 2.53 and Eq. 2.54, where a and b correspond to 2.62 and 2.63. Note, that in convention used here the oscillations are observed at negative current [16]. Therefore, further study is limited to negative currents, and for simplicity of notation the absolute value of current is be presented hereafter.

Following parameters have been used for the systematic study: the anisotropy constant $\mathrm{K}_{\mathrm{u}}=3.46 \cdot 10^{3} \mathrm{~J} / \mathrm{m}^{3}$, exchange constant $\mathrm{A}=1.3 \cdot 10^{-11} \mathrm{~J} / \mathrm{m}$, damping constant $\alpha=0.01, \mathrm{M}_{\mathrm{s}}=6.9 \cdot 10^{5} \mathrm{~A} / \mathrm{m}$, and $\mathrm{M}_{\text {sfixed }}=1.4 \cdot 10^{6} \mathrm{~A} / \mathrm{m}$. The computational area has been discretized into $5 \times 5 \times 4 \mathrm{~nm}^{3}$ cells.

All the important parameters for transport calculations are gathered in Tables 3.1 and 3.2 below. 
Tab. 3.1: Parameters for transport calculations.

\begin{tabular}{cccc}
\hline \hline & $\mathrm{Cu}$ & $\mathrm{Co}$ & $\mathrm{Py}$ \\
\hline bulk resistivity $(\mu \Omega \mathrm{cm})$ & 0.5 & 5.1 & 16 \\
asymmetry factor & 0.0 & 0.51 & 0.77 \\
spin diffusion length $(\mathrm{nm})$ & 1000 & 60 & 5.5 \\
\hline
\end{tabular}

Tab. 3.2: Interface parameters for transport calculations.

\begin{tabular}{ccc}
\hline \hline & $\mathrm{Co} / \mathrm{Cu}$ & $\mathrm{Py} / \mathrm{Cu}$ \\
\hline resistance $\left(f \Omega m^{2}\right)$ & 0.5 & 0.5 \\
asymmetry factor & 0.77 & 0.7 \\
real mixing conductance $\left(\left(f \Omega m^{2}\right)^{-1}\right)$ & 0.542 & 0.39 \\
imaginary mixing conductance $\left(\left(f \Omega m^{2}\right)^{-1}\right)$ & 0.016 & 0.012 \\
\hline
\end{tabular}

\subsection{Critical angle study}

In this section the effect of variation of the spin diffusion length $l_{\mathrm{sf}}$ on the resulting profile of the ST is investigated. Determination of the STT angular variation requires a number of parameters. Some of them can be extracted from CPP-GMR experimental data (spin diffusion length, bulk and interfacial resistivity, spin asymmetry), while the others can be provided by $a b$ initio calculations (real and imaginary parts of the mixing conductance). Thus, fabrication methods, external conditions, and defects of various types determine the characteristics of the resulting STT i.e. its amplitude and the critical angle. The latter defines the angle at which the torque vanishes.

As shown in Fig. 3.2a the amplitude of ST does not depend on the $1_{\mathrm{sf}}$, however, its decrease leads to the decrease of the critical angle. The inset specifies the largest, in black, and smallest, in green, $l_{\text {sf }}$ used for the calculations with red being intermediate value.

Once calculated, the torque profiles are employed for the study of ST 

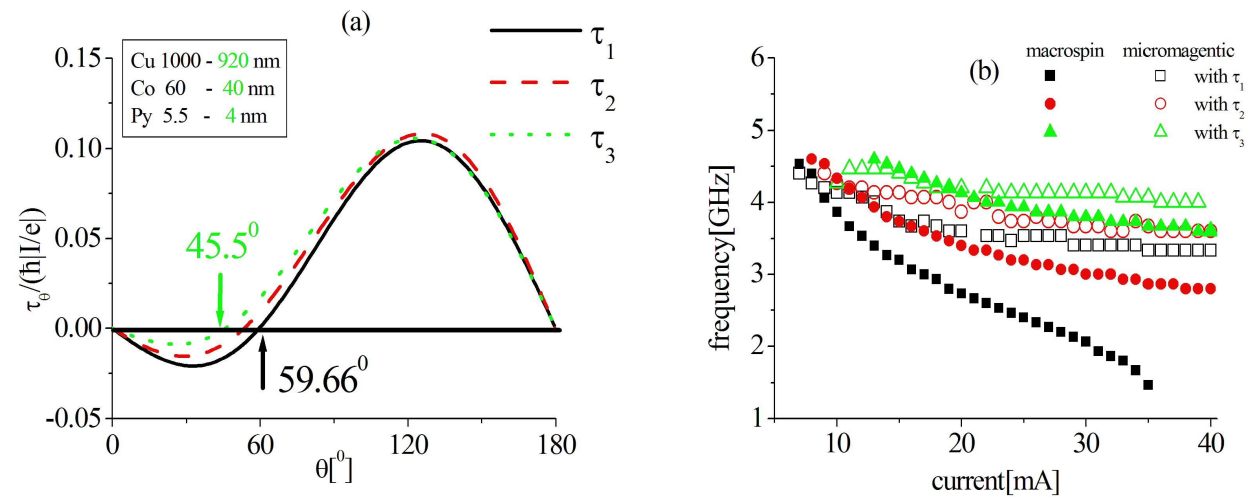

Fig. 3.2: (a) Angular variation of STT for different values of $1_{\mathrm{sf}}$. Solid black line corresponds to the first and dotted green to the third $l_{\text {sf }}$ from the inset while dashed red to the intermediate value (not given). (b) Frequency of microwave oscillations as a function of current for the situations corresponding to (a). The open symbols correspond to the results of micromagnetic study while the full ones to macrospin model.

driven oscillations supported in the pillar. The following convention is used throughout this paper: open symbols correspond to results of micromagnetic study while full ones to macrospin approximation. In this study initial parallel (P) magnetic configuration was assumed and the current was increased step-wise (with the step $\Delta I$ ) i.e. as the initial state for simulation at a given current $I+\Delta I$ the state the system arrived at in the preceding step of simulations is assumed. The analysis shows that generally a low angle IPP + mode is supported and the associated frequency redshift with current is faster in the macrospin than in the micromagnetic case. Note that in the latter the coherence is partly lost due to the appearance of the inhomogeneities in the magnetic configuration. This leads to less pronounced variation of averaged magnetization and finally to 'retarded' micromagnetic redshift.

Let us consider the situation corresponding to the first set of parameters. Interestingly, a cut-off current is then reported within the macrospin model, above which no oscillations are supported and a stable static spin-up state 
is observed [27]. However, for the $2^{\text {nd }}$ and $3^{\text {rd }}$ sets of parameters (smaller $l_{\text {sf }}$ ), the critical angle is decreased and the spin-up static state is no longer observed in the dynamic study, see Fig. 3.2b. Accordingly, the red-shifting frequency branch was observed up to largest studied current (I > $40 \mathrm{~mA}$, not shown).

The appearance of self sustained oscillations and of the static state is in agreement with the conclusion in Ref. [16] that both are macrospin solutions of the Landau-Lifshitz-Gilbert equation with the wavy torque included. The static state, however, has neither been observed in micromagnetic simulations nor reported experimentally. Clearly, its appearance is a feature of the macrospin model, which can be explained as follows. When the current increases, the amplitude of the IPP increases as well and, therefore, the effective angle between the magnetization orientation of the free and fixed layer increases. At some point the critical angle is reached and, as a result, the current-induced torque vanishes. Thus, the dynamics is determined mainly by the demagnetizing field (the main contribution to the effective field). The magnetization of the free layer becomes then aligned with the effective field and finally the static state is stabilized $(\mathrm{I}=35 \mathrm{~mA})$. No such state has emerged from micromagnetic study, where the presence of inhomogeneities favors less coherent dynamics and the critical angle can not be reached exactly. Additionally, one should note that at a lower critical angle (third set), the quantitative difference in the frequency response obtained in frames of both macrospin and micromagnetic models decreases. Obviously, as we deal with the IPP modes, the lower the critical angle, the more uniform the magnetization state of the free layer is when reaching this angle (more coherent dynamics) and, therefore, the more consistent are the predictions of both models.

The existence of the peculiar static state in macrospin model has its further consequences i.e. as the current is decreased the OPP mode is triggered, as shown in Fig. 3.3. It is convenient to locate each mode in the torque shape 
diagram as indicated in the inset, where $\mathrm{IPP}_{+} / \mathrm{OPP}$ corresponds to low/large angle range. This hysteretic behavior (also reported in Refs. [27, 28]) has not been observed in the micromagnetic study, where no cut-off current has been reported. Moreover, there is no experimental evidence for the appearance of the spin-up static state. On the contrary, the OPP mode was reported in Ref. [17].

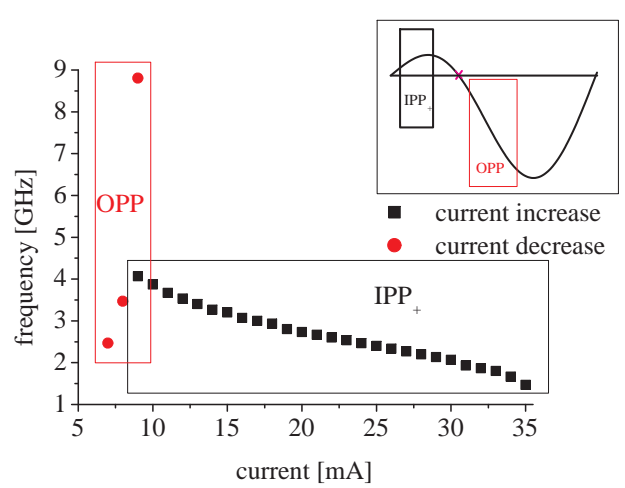

Fig. 3.3: The effect of current increase and decrease in absence of external magnetic field (macrospin simulations). Current increase leads to the appearance of the in-plane precession at the threshold of $9 \mathrm{~mA}$ and a 'cut-off' current of $35 \mathrm{~mA}$, at which a static stable state is reached (spin-up state: $\left.\left\langle m_{z}\right\rangle=1\right)$. Starting from this state and decreasing the current, the OPP is triggered in a small range of currents. In the inset the IPP and OPP are located schematically in the torque diagram.

It should be emphasized that the maximum frequency of the macrospin OPP mode is twice as large as that in the case of the IPP mode. This difference can be explained as follows. The precession axis is determined by the demagnetizing field, as it is the main contribution to the effective field. The demagnetization field reads $\mathbf{H}_{d}=-M_{s}\left(N_{x} m_{x}, N_{y} m_{y}, N_{z} m_{z}\right)$, and depends on the demagnetization factors $\mathrm{N}_{\mathrm{x}}, \mathrm{N}_{\mathrm{y}}, \mathrm{N}_{\mathrm{y}}$, and on the magnetization state. As a result, in the case of IPP mode, where $m_{x}>m_{y}, m_{z}$, the 
demagnetizing field points mainly along the $x$ direction, leading to rough estimation $\mathbf{H}_{d}=-M_{s}\left(N_{x} m_{x}, 0,0\right)$. On the contrary, for the OPP mode $\mathrm{m}_{\mathrm{z}}>>\mathrm{m}_{\mathrm{x}}, \mathrm{m}_{\mathrm{y}}$ and analogously $\mathbf{H}_{d}=-M_{s}\left(0,0, N_{z} m_{z}\right)$. The demagnetizing factors for the ellipse under study are $\mathrm{N}_{\mathrm{x}}=-5,69 \cdot 10^{-2}, \mathrm{~N}_{\mathrm{y}}=-8.9 \cdot 10^{-2}$, and $\mathrm{N}_{\mathrm{z}}=-0.85$. Since the frequency is proportional to the effective field, the macrospin frequency in the OPP regime reaches high values owing to the fact that $\mathrm{N}_{\mathrm{z}}>>\mathrm{N}_{\mathrm{x}}$. In a real pillar there are other contributions to the effective field, in particular the exchange field, which counterbalances the effect of the demagnetizing field and leads to lower frequencies of the OPP mode.

For future discussion, it is convenient to explain in detail the ST diagram presented in Fig. 3.4 where clear division between certain regions is done. Region I was shown to support IPP + mode until macrospin spin-up state was reached, marked as a circle. The OPP mode was associated with region II as the current was decreased and so far no dynamics was observed in region III.

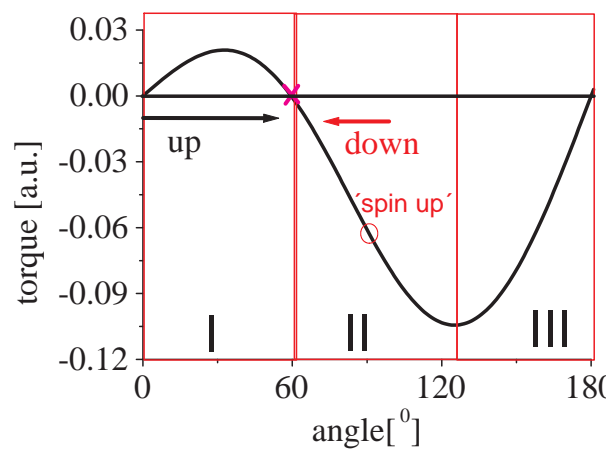

Fig. 3.4: The torque acting on the free layer normalized to $\hbar j /|e|$ for negative current density $(j<0)$. This way the IPP is associated to region I the OPP to region II. The arrows indicates direction of current change. The III region is to be investigated later. 


\subsection{Oscillations in presence of external field.}

As it turned out in the previous section, manipulation of the critical angle does not result in the appearance of physically justified mechanism for OPP. Therefore, a moderate external field ( up to $90 \mathrm{mT}$ ) is investigated as a possible cause for the triggering such oscillations. Fields ranging from 10 up to $90 \mathrm{mT}$ are applied at different angles $\alpha$ with respect to the ellipse plane (with the in-plane component along the easy axis) since from the practical point of view only low field regime is interesting. In the following, further division is adapted, ordering the effects observed at low (20 mT), intermediate (36 mT) and large fields $(90 \mathrm{mT})$.

\subsubsection{Field orientation $0^{\circ}<\alpha<90^{\circ}$}

The analysis starts with the field orientation between IP, $\alpha=0^{\circ}$, and OP, $\alpha=90^{\circ}$. For all studied fields in this range of orientations, i.e. for $10 \mathrm{mT}$, $20 \mathrm{mT}, 36 \mathrm{mT}$ and $90 \mathrm{mT}$, one obtains a qualitative agreement between the predictions of macrospin and micromagnetic models. The system supports small angle IPP + mode, which is sensitive to the strength of the IP field as this orientation of the external field coincides with the direction of the effective field, and one observes frequency redshift with current (Fig. 3.5). As for the dependence on magnetic field, one observes frequency blueshift with the IP field strength (not shown). On the other hand, the increase of the field applied in the OP direction does not influence the frequency of the observed mode (not shown), but again the macrospin cut-off current is reported. Thus, moderate field applied at an angle from the first octant of the coordinate system supports precession which is qualitatively similar to the mode observed in absence of external field, restricting the dynamics to the region corresponding to $\mathrm{IPP}_{+}$as denoted in the inset of Fig. 3.5. 


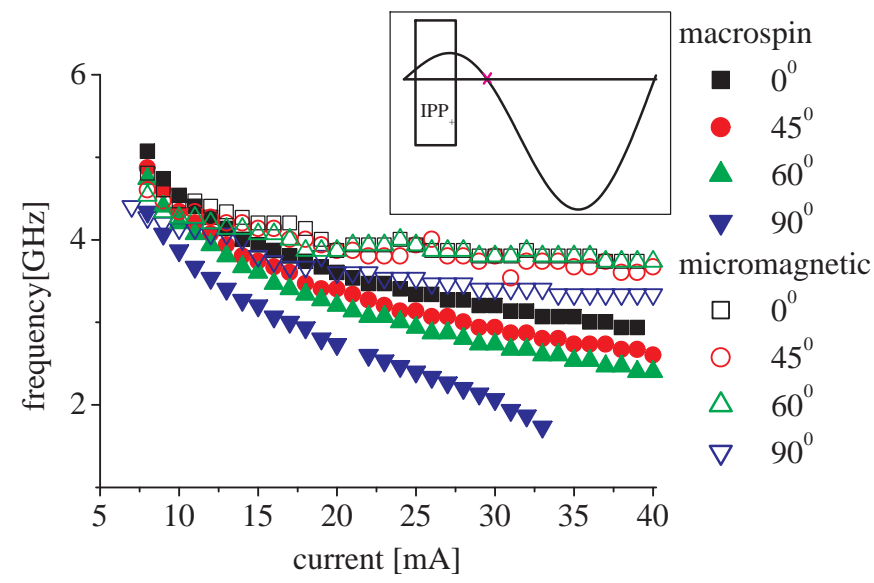

Fig. 3.5: Frequency response to current at $B_{\text {ext }}=10 \mathrm{mT}$ applied at different angles. The angle is measured from the ellipse plane, i.e. $0^{\circ}$ corresponds to inplane field and $90^{\circ}$ to out-of-plane field. The inset locates the oscillation mode in the torque shape diagram.

\subsubsection{Low fields; $\alpha>90^{\circ}$}

In the search for the OPP mode one should consider fields applied at larger angles $\left(\alpha>90^{\circ}\right)$ in order to favor the OP alignment of the effective field. In the following $120^{\circ}, 150^{\circ}$, and $180^{\circ}$ angles are investigated. For the smallest field studied $(10 \mathrm{mT})$, the dynamic response does not differ qualitatively from that already discussed at $0 \mathrm{mT}$. Hence, for larger fields $(20 \mathrm{mT})$ some qualitative changes can be observed. As shown in Fig. 3.6a for the field of $20 \mathrm{mT}$ applied at $120^{\circ}$ and $150^{\circ}$, the redshift tendency continues to be supported. However, in micromagnetic simulations chaotic oscillations are observed at large currents (above chaotic oscillations current threshold). The emerging frequency spectra become very noisy, the exact frequency peak can not be defined anymore and, therefore, the results are presented only below this threshold. At smaller angles, the threshold current for transition to chaotic regime is larger, i.e. at $\alpha=120^{\circ}$ the chaotic oscillations are 
found at $\mathrm{I}=28 \mathrm{~mA}$, while at $\alpha=150^{\circ}$ and $\alpha=180^{\circ}$ they appear already at $\mathrm{I}=16 \mathrm{~mA}$ (Fig. 3.6a). Analogously, macrospin approximation leads to the cut-off current larger at $\alpha=120^{\circ}(\mathrm{I}=16 \mathrm{~mA})$ than at $\alpha=150^{\circ}$ $(\mathrm{I}=11 \mathrm{~mA})$. The combined influence of the field and spin torque still supports only the $\mathrm{IPP}_{+}$mode, restricting the dynamics to relatively small current range. New situation arises in the macrospin approach when the field is applied at $\alpha=180^{\circ}$. At the threshold of $\mathrm{I}=10.8 \mathrm{~mA}$, a transition between a low angle $\mathrm{IPP}_{+}$and a large angle (inset Fig. 3.6) $\mathrm{OPP}_{+}$takes place (redshift and blueshift, respectively). However, this transition is not observed in the micromagnetic model, where the supported dynamics is restricted to the $\mathrm{IPP}_{+}$mode.

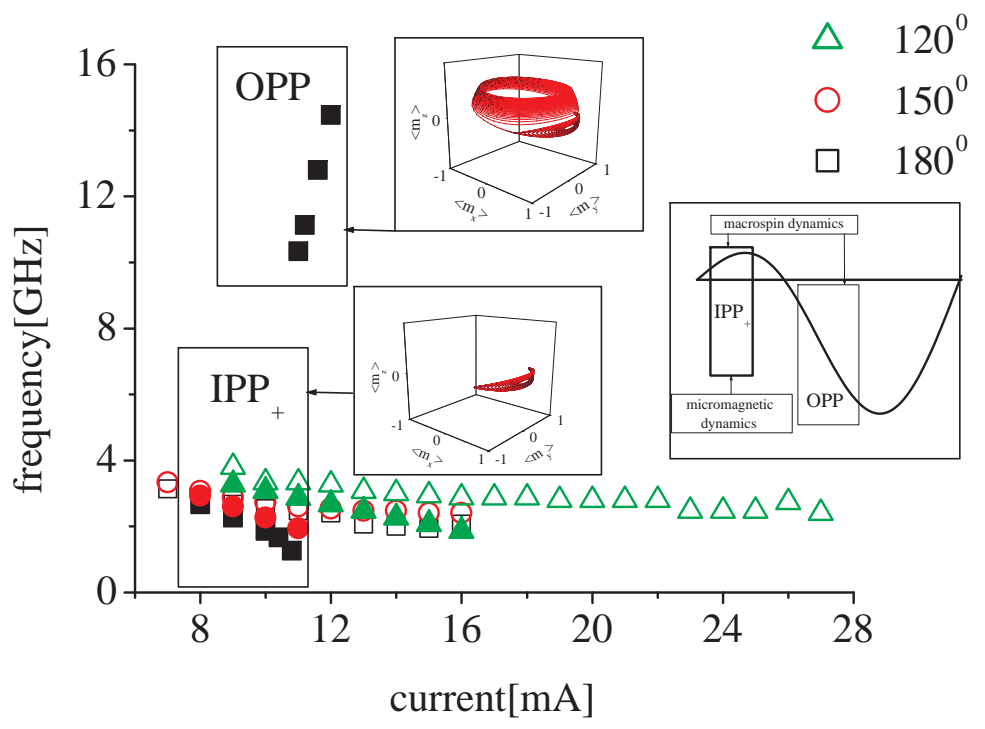

Fig. 3.6: Frequency as a function of current for different angles $\alpha>90^{\circ}$ and for the applied field of $20 \mathrm{mT}$. Insets: macrospin small angle orbit of IPP+ mode at $\mathrm{I}=10.8 \mathrm{~mA}$, large angle $\mathrm{OPP}$ orbit at $\mathrm{I}=11 \mathrm{~mA}$, and modes located in the torque diagram. 


\subsubsection{Intermediate fields; $\alpha>90^{\circ}$}

Increasing the field strength up to $36 \mathrm{mT}$ leads to new features (Fig. 3.7). The micromagnetic model again predicts frequency redshift with current and a chaotic oscillation regime. However, the field contribution qualitatively

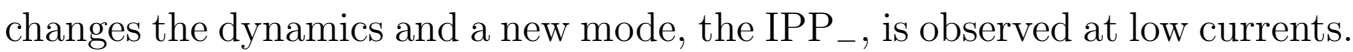
Clearly, the applied field favors the antiparallel (AP) configuration, shifting thus the system towards large angle IP dynamics.

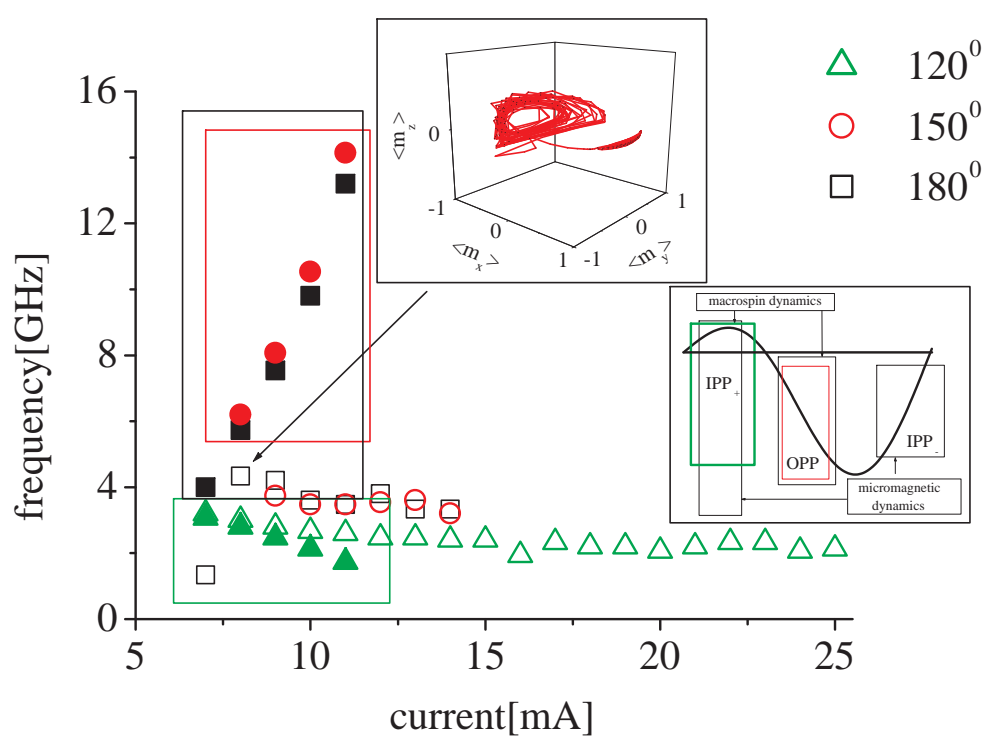

Fig. 3.7: Frequency as function of current for different angles $\alpha>90^{\circ}$, and for the applied field of $36 \mathrm{mT}$. Insets: micromagnetic change of the orbit at $180^{\circ}$ corresponding to the transition between the modes, and location of the excited modes in the torque shape diagram.

The previously observed IPP + mode can be found as the main mode at $120^{\circ}$, and its trace is also visible at the oscillation threshold current of $7 \mathrm{~mA}$ at $180^{\circ}$. At larger currents, however, it is followed by the IPP_ dynamics. 
As can be seen in the inset of Fig. 3.7, this transition between the modes is reflected in the change of the orbit. Note, that quantitatively the IPP - and $\mathrm{IPP}_{+}$are the same mode yielding similar frequency and, therefore, can be distinguished only by the visualization of the orbit.

In the macrospin approximation, on the other hand, the IPP + mode emerges at $120^{\circ}$, which is consistent with the above discussed micromagnetic case. Thus, the field applied at either $150^{\circ}$ or $180^{\circ}$ leads to the sole appearance of the $\mathrm{OPP}_{+}$mode. Unlike for smaller fields, here the OPP regime is not preceded by a small angle precession regime.

At this point it is worth considering a particular geometry issue. As presented in Fig. 3.8 the design of pinned layer can be twofold: an etched or an extended structure. In the latter the influence of the magnetostatic interlayer coupling field (ICF) can be then neglected. On the contrary, in the first case it should be added to the effective field. Note that $36 \mathrm{mT}$ applied at $180^{\circ}$ ia approximately the stray field from the PL (Fig. 3.1). In other words, the OPP is supported in etched pillars, where stray field plays important role, in absence of external field (within the macrospin frame). Nevertheless, this regime does not appear in the micromagnetic study. Thus, the micromagnetic and macrospin dynamics at field applied at the studied angles are qualitatively different, supporting modes of different kinds.

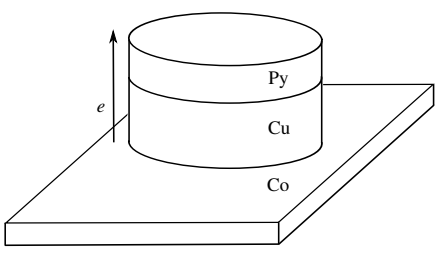

(a)

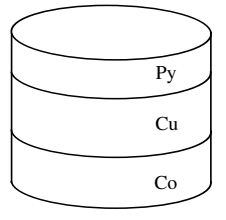

(b)

Fig. 3.8: Geometry of a pillar with (a) an extended and (b) etched fixed layer. The interlayer coupling field in (a) can be neglected. 
3.3.4 Large fields; $\alpha>90^{\circ}$.

So far, macrospin model resulted in the following: the low angle $\mathrm{IPP}_{+}$at $10 \mathrm{mT}$, the transition from low angle $\mathrm{IPP}_{+}$to large angle OPP at $20 \mathrm{mT}$, and the direct appearance of the OPP at $36 \mathrm{mT}$. On the other hand, micromagnetically the $\mathrm{IPP}_{+}$and the IPP $\mathrm{I}_{-}$were reported, and no OPP mode has been identified. Therefore, in this section even larger magnetic fields are studied. Increasing the field up to $90 \mathrm{mT}$ revealed some interesting behavior. Still, micromagnetically only the red-shifting $\mathrm{IPP}_{-}$is supported up to a certain current, at which a chaotic oscillation regime is found (not shown). On the contrary, some complicated transitions are observed in the macrospin model. First of all, the field of $90 \mathrm{mT}$ is strong enough to influence the effective field direction, so the low angle IPP + mode is not present anymore. Instead, different modes associated with different orbits, as shown in Fig. 3.9, are found. The field applied at $180^{\circ}$ results in a single transition at $\mathrm{I}=8.0 \mathrm{~mA}$. To get a deeper insight into the origin of this effect one shall investigate the averaged magnetization orbital evolution. Figures $3.9 \mathrm{~b}, \mathrm{c}, \mathrm{d}$, e present the orbit at $\mathrm{I}=3 \mathrm{~mA}, 6 \mathrm{~mA}, 8 \mathrm{~mA}$ and $8.4 \mathrm{~mA}$, respectively. At $\mathrm{I}=3 \mathrm{~mA}$, the $\mathrm{IPP}_{-}$ mode with associated clamshell orbit (Fig. 3.9b) is reported. As the $\left\langle m_{x}\right\rangle$ amplitude increases, the orbit narrows (Fig. 3.9c) to form the open clamshell orbit just before the transition at $\mathrm{I}=8.0 \mathrm{~mA}$ (Fig. 3.9d), which separates the regions of frequency redshift and blueshift with current. The transition leads to the appearance of the $\mathrm{OPP}_{-}$mode (with $\left\langle m_{z}\right\rangle$ oscillating in the negative range). This mode is characterized by the $\left\langle m_{z}\right\rangle$ amplitude decreasing with increasing current and large angle orbit, as presented in Fig. 3.9e. Clearly, this transition (at $90 \mathrm{mT}$ ) is different from the ones observed at smaller fields (20 $\mathrm{mT}$ and $36 \mathrm{mT}$ ), since at large field no small angle orbit $\left(\mathrm{IPP}_{+}\right)$is present at all. Moreover, the field of $90 \mathrm{mT}$ applied at $120^{\circ}$ or $150^{\circ}$ results in the appearance of two blue-shifting branches in the frequency response. For the case of $120^{\circ}$, two transitions at currents of $6.0 \mathrm{~mA}$ and $8.4 \mathrm{~mA}$ are present. 
As the angle is increased to $150^{\circ}$, the transition currents are shifted towards larger values of $7.8 \mathrm{~mA}$ and $8.8 \mathrm{~mA}$, respectively. The origin of the multiple transitions in both cases is the same and it shall be explained on the example of $150^{\circ}$. As previously, the first stage is the red-shifting IPP mode. As the current is increased, an open clamshell orbit forms. The first transition is found at $7.8 \mathrm{~mA}$, and up to $8.8 \mathrm{~mA}$ the $\mathrm{OPP}_{+}$mode is supported, which corresponds to the orbit depicted in Fig. 3.9f. The associated amplitude decreases as the current increases. However, at $8.8 \mathrm{~mA}$ an orbit equivalent to the one presented in Fig. 3.9e is formed by shifting $\left\langle m_{z}\right\rangle$ downwards and opening a new branch in the frequency response diagram $\left(\mathrm{OPP}_{-}\right)$. In other words, at the critical current of $8.8 \mathrm{~mA}$, the system dynamics depends on the initial demagnetizing field, which is driven directly by the magnetization state and indirectly by the direction of current sweeping. These transitions can be located in the torque diagram as indicated in the inset.

\subsection{Oscillations in absence of external field}

The conclusion of the previous paragraphs is that the experimentally observed OPP has not been confirmed numerically yet. Therefore, the model is now refined and several detailed issues are investigated.

First of all, attention is paid back to the exact geometry i.e. the etched and extended geometry of the pinned layer as in Fig. 3.8. Etched structure gives rise to some significant magnetostatic field. Therefore, in the following section the potential difference arising from this complementary field is to be studied.

Second, the initial state and its influence on the dynamic evolution of the oscillations is determined. So far only initial $\mathrm{P}$ state has been considered and thus now the study is extended to the antiparallel (AP) state.

Third, repeating discrepancy between the results of macrospin and mi- 

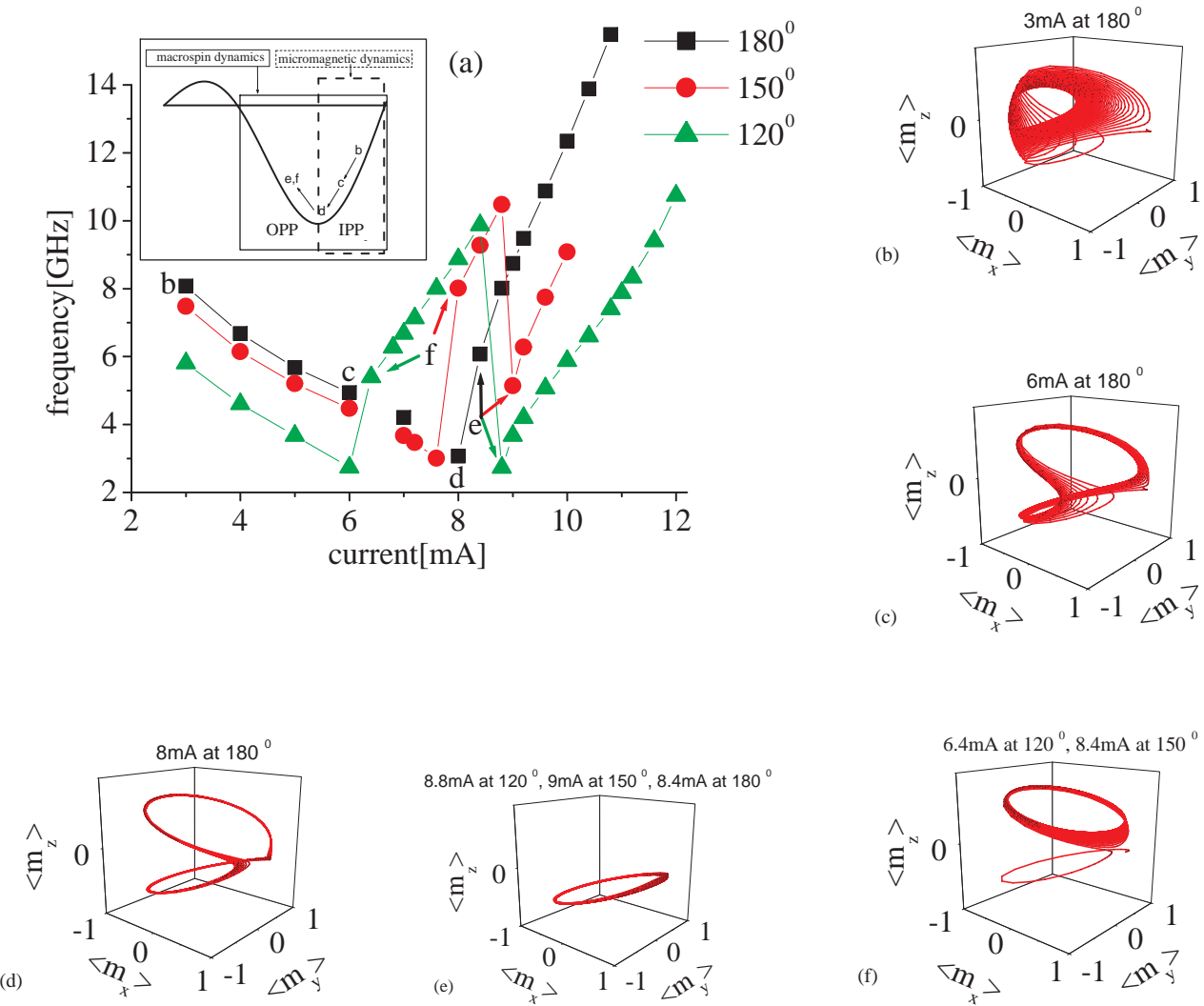

Fig. 3.9: Frequency as a function of current for different angles $\alpha>90^{\circ}$, and for applied field of $90 \mathrm{mT}$ (a). At $180^{\circ}$ a single IPP-OPP transition appears at $\mathrm{I}=8.0 \mathrm{~mA}$, which is associated with the orbit change from clamshell (b) at $3 \mathrm{~mA}$, narrowing (c) at $6 \mathrm{~mA}$, and (d) $8 \mathrm{~mA}$, and finally falling into the $\mathrm{OPP}_{-}$large angle orbit (e) at $8.4 \mathrm{~mA}$. For $120^{\circ}$ and $150^{\circ}$, the frequency exhibits two blue-shifting branches. The first transition is visible at $\mathrm{I}=6.4 \mathrm{~mA}$ and $\mathrm{I}=8.4 \mathrm{~mA}$ for $120^{\circ}$ and $150^{\circ}$, respectively, and is associated with the orbit change from clamshell as in (d) to large angle $\mathrm{OPP}_{+}$as in (f). The threshold for the second blue-shifting branch is $8.8 \mathrm{~mA}\left(120^{\circ}\right)$ and $9.0 \mathrm{~mA}\left(150^{\circ}\right)$. It represents an equivalent orbit analogous to (e), and its appearance is triggered by the initial magnetization state. The instead locates the modes and corresponding orbits. 
cromagnetic model lead to the reconsideration of some factors entering the latter.

\subsubsection{Extended geometry, AP initial state}

The study starts with a pillar structure having an extended fixed layer. Since the anisotropy of Py is small the dynamics is driven mainly the self magnetostatic field and ST. These two contributions are closely related. The first one, depending on the initial magnetization configuration, defines the initial angle between the magnetic moments of the layers. This, in turn, determines the initial torque sign and strength. In the following, two situations are analyzed - when the final state at a given simulation step is assumed for the initial state in the next current step, and when the AP initial state was assumed at each current step. Consider first the former situation. As a reminder, starting from $\mathrm{P}$ state revealed that the $\mathrm{IPP}_{+}$mode supported in region I (Fig. 3.4) has been found in both macrospin and micromagnetic model and the static state and OPP in the region II have been found only in the macrospin approximation. Thus, in order to investigate modes supported in region III, simulations assuming AP initial configuration are required.

As the current is increased, the micromagnetic simulations reveal a fast red-shifting branch in the range marked as 1 in Fig. 3.10 (open squares), corresponding to the $\mathrm{IPP}_{-}$mode (precession around the $-x$ axis). Fast amplitude increase with increasing current leads to switching towards the $\mathrm{P}$ state and damped oscillations stabilizing the $\mathrm{P}$ state are observed (range 2). However, as certain threshold current $(\mathrm{I}=7 \mathrm{~mA})$ is reached ( $\mathrm{P}$ is now the initial state), the STT destabilizes the P state counterbalancing damping and the second red-shifting branch, IPP ${ }_{+}$, is observed (range 3). This branch is, thus, equivalent to the corresponding one in Fig. 3.2b. Micromagnetically, the only difference between starting with initial P or AP state (open circles and open squares in Fig. 3.10, respectively) is the appearance of the first fast red-shifting IPP_ branch. 


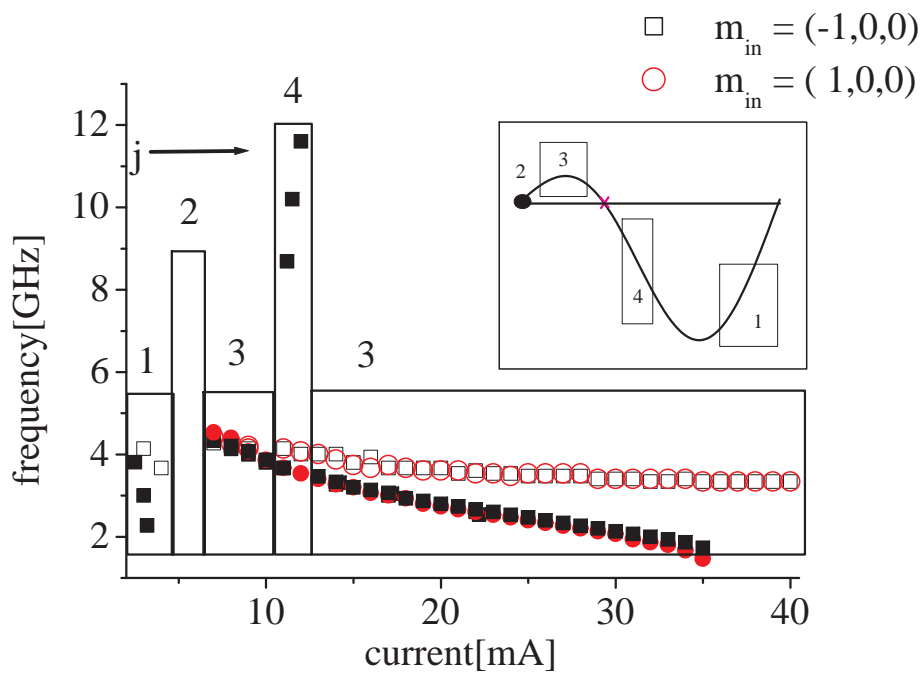

Fig. 3.10: The influence of the initial magnetization state on the dynamic behavior of the system and the corresponding spots in torque diagram (inset). Circles refer to P, whereas squares to AP initial state. At low currents, IPP _ mode (close to the AP direction) is found (marked as 1). As the current increases, the angle of the orbit increases as well, and $\left\langle m_{x}\right\rangle$ switches towards the P state. No self sustained oscillations are observed in the range 2. Further current increase leads to the onset of the second red-shifting branch, $\mathrm{IPP}_{+}$, marked as range 3 . Additionally at a certain threshold current, the combined effect of self magnetostatic field of the AP initial state and negative torque sign can trigger the OPP in the macrospin model, marked as 4 .

Similar situation appears in the macrospin simulations. Starting from the AP initial state and increasing current the IPP - mode (full squares in the region 1 ) is found and then the red-shifting mode $\mathrm{IPP}_{+}$observed before.

Consider now the case with the AP state assumed at each current step. As before, macrospin model yields both IPP - and IPP + modes, except for the region 4 (full squares), where now a new mode is visible. This is the OPP 
mode, equivalent to that observed when starting simulation from the static point, as in previous paragraphs. Thus, if the current is large enough and the simulations are initialized with the AP state, the system might be forced to move into the region II (Fig. 3.4), leading to the appearance of OPP marked as range 4 in Fig. 3.10 (full squares). Earlier in this section it has been shown that after crossing from region I through the critical angle into the region II, the static state was observed. Here, though, dynamics in the region II is forced by the initial configuration and therefore the OPP can be observed. In other words, in the single domain approximation region I supports IPP ${ }_{+}$, region II supports static state or OPP (depending on the preceding configuration), whereas in region III the IPP _ mode can be observed. This result is consistent with the one reported in [27]. However, no OPP mode was found in the micromagnetic simulations.

\subsubsection{Etched geometry}

In the etched geometry shown schematically in Fig. 3.8b, the ICF can no longer be neglected. It has been calculated micromagnetically, and (neglecting large OP edge values) was estimated to be an average IP field of $-36 \mathrm{mT}$. This is a significant contribution and, therefore, dynamics different from that obtained for extended structures can be expected.

The dynamic response reveals now some new interesting features. Simulations for increasing as well as decreasing current have been performed. As the initial state for a particular simulation step the state reached in the preceding step is assumed. As before, the starting configuration (the first simulation step) was either P or AP state.

\section{Initial $P$ state}

Consider first macrospin analysis for increasing current. The initial $\mathrm{P}$ state leads now to the steady OPP, which appears at $10.4 \mathrm{~mA}$ when the current 
is increased, as indicated in Fig. 3.11 (see the upper part of the figure, with the corresponding range for the observed mode marked with dotted lines). As the threshold current for the OPP is reached, the blue-shifting branch appears in the range 2. This OPP is not preceded by any IPP oscillations in the range 1 , because the additional contribution from ICF places the system directly in region II (as defined in Fig. 3.4), i.e. the ICF favors AP configuration whereas the STT destabilizes it. As the frequency of the OPP mode increases with increasing current, the corresponding amplitude decreases and the angle between the magnetic moments of both layers approaches the critical angle. When this angle is reached, the static state discussed in the preceding subsection is observed (range 3). Now, decreasing current with the system initially in the static point yields dynamic range marked with solid lines in Fig. 3.11 (bottom part). The OPP appears then in the corresponding range 1 at $\mathrm{I}=9.8 \mathrm{~mA}$, and the amplitude and $\theta$ increase as the current decreases. The torque minimum is then passed and the system moves to the region III, which results in the appearance of the IPP - mode in the range 2 , where the amplitude decreases (with decreasing current), which results effectively in the frequency redshift with current. The asymmetry in the macrospin frequency response to increasing and decreasing current is clearly a consequence of the torque asymmetry, the existence of a critical angle, and irreversibility of the transition from region I to region II.

The dynamics in the micromagnetic model is simpler. Due to the effect of ICF, the system directly switches to the region III, and only one red-shifting branch $\mathrm{IPP}_{-}$is observed. A part of this branch is observed for increasing current and the other part for decreasing current, as is clearly visible in Fig. 3.11. For decreasing current this mode is qualitatively similar to the macrospin mode observed in range 2 . 


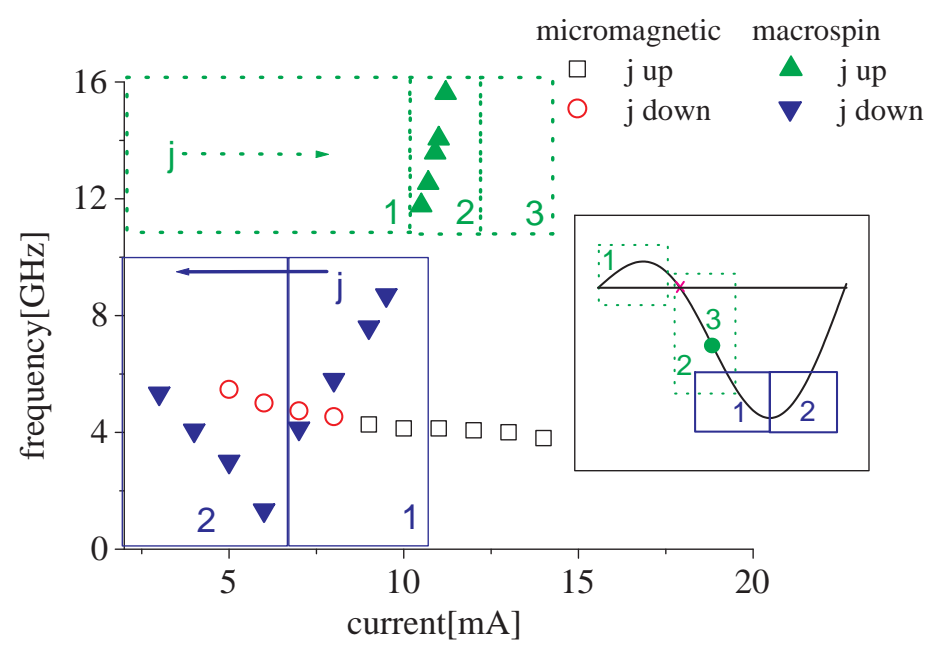

Fig. 3.11: The influence of current increase and decrease in the etched pillar geometry on the dynamic response of the system with the initial $\mathrm{P}$ state. The corresponding torque diagram is shown in the inset. Note asymmetry in the macrospin frequency response - OPP with current increase and transition from OPP to IPP _ with current decrease are observed. This hysteretic behavior originates from the asymmetric torque angular variation and the existence of the critical angle making dynamic transition between the region I and II of Fig. 3.4 prohibited.

\section{Initial AP state}

As discussed above, starting from the initial $\mathrm{P}$ state leads to a hysteretic dependence in the frequency response within the macrospin approximation. Consider now the situation with the initial AP state. When the current is increased (see the area bounded by the dotted line in Fig. 3.12), the system is directly placed in the region III, supporting red-shifting branch (IPP_) in the range 1 (inset Fig. 3.12). As the amplitude increases with increasing current, transition to range 2 , where the OPP mode is supported, is observed. In the torque diagram this is equivalent to the transition from region III over 
the torque minimum to the region II (Fig. 3.4). Since the critical angle is not crossed, this transition stays reversible and no hysteretic behavior in the frequency response is observed i.e. both: red-shifting and blue-shifting branch are observed for current being decreased and increased.

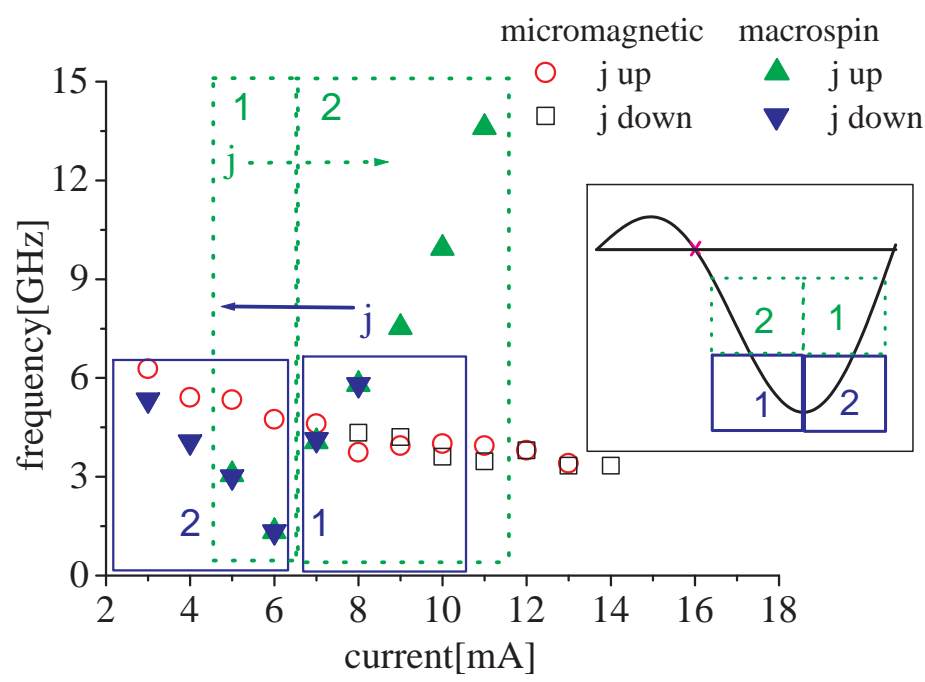

Fig. 3.12: The same as in Fig. 3.11, but for the AP initial state. Micromagnetically only the IPP_ mode in the region III is observed.

As before, no OPP was found in the micromagnetic simulations. For both increasing and decreasing current only the IPP - mode is observed. This indicates that the system supports stable oscillations only in region III.

\subsubsection{Influence of the exchange field}

An open question is why the OPP modes obtained in the macrospin model and also reported experimentally [18], have not been found in the micromagnetic simulations presented above. As it has been shown, to observe the OPP-associated blueshift, one has to force the system dynamics in the region II (Fig. 3.4). Moreover, it has been concluded that the appearance 
of the macrospin spin-up state was a consequence of the crossing over the critical angle. This has not been reached micromagnetically due to the inhomogeneous nature of the magnetization (finite exchange field) in the model. Therefore, one should expect that the appearance of OPP in micromagentic model is hindered by the finite exchange field, and that increase of the exchange constant should lead to the convergence of both models. Using the bulk exchange constant for thin films might cause a significant underestimation of real exchange fields in these structures. Larger values of exchange constants, as compared to the standard bulk ones, have been reported in Py dots [59] and thin films [60].

\section{The extended geometry}

In the extended geometry magnetization dynamics for increasing current and for the following values of the exchange constant: $0.75 A_{0}, 2.5 A_{0}, 3 A_{0}$ has been investigated. The frequency-current behavior with $\mathrm{A}=3 A_{0}$ is compared to the results of macrospin model in Fig. 3.13. Clearly, increasing the exchange constant changes the slope of micromagnetic frequency redshift towards macrospin results. Thus, one may conclude that the finite exchange energy, favoring inhomogeneous magnetization state, drives less coherent dynamics and therefore causes this slope difference.

\section{Etched geometry}

In this geometry (ICF included) so far micromagnetically induced dynamics in the region III and the associated frequency redshift, as well as the macrospin dynamics in the region II supporting the OPP mode with the associated blueshift have been reported. Still, change of the exchange constant could, at least in principle, shift micromagnetic dynamics to region II. The micromagnetic temporal evolution of the averaged magnetization at 


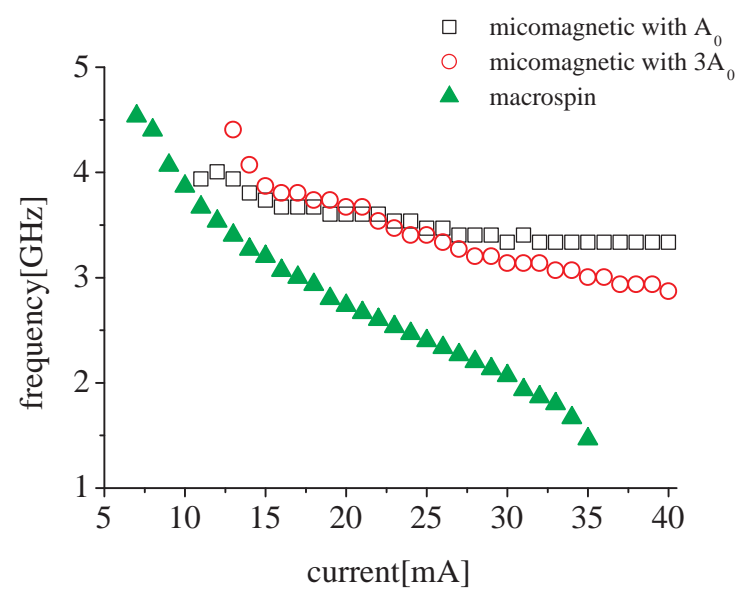

Fig. 3.13: The dynamic response of the system for two different values of the exchange constant, compared to the macrospin results. Micromagnetically faster redshift is observed for larger exchange constant, as it favors more homogeneous magnetization configuration i.e. more coherent dynamics.

$\mathrm{I}=13 \mathrm{~mA}$ for $\mathrm{A}=A_{0}$ and $\mathrm{A}=2.5 A_{0}$ results in different orbits, Fig. 3.14a and Fig. 3.14b, respectively. Clearly, in the first case the ICF places the system in region III forcing IPP _ dynamics. However, as the exchange field is increased, which favors uniform magnetization, an open clamshell orbit is formed (Fig. 3.14b) shifting the dynamics toward the border between regions III and II. One should note that the cross-over between the regions is impossible in this geometry as the ICF has a significant impact on the effective field hindering the appearance of OPP mode.

\subsubsection{Comparison to experimental data}

Up to now, no OPP (supported in the dynamic region II) has been predicted micromagnetically, even though such modes were reported experimentally [18] in extended structures. However, previous paragraphs have given some 


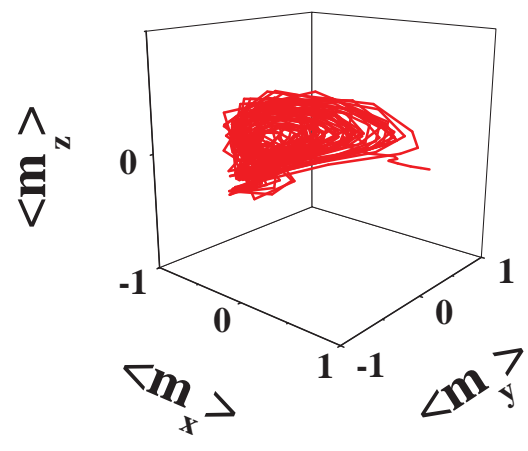

(a)

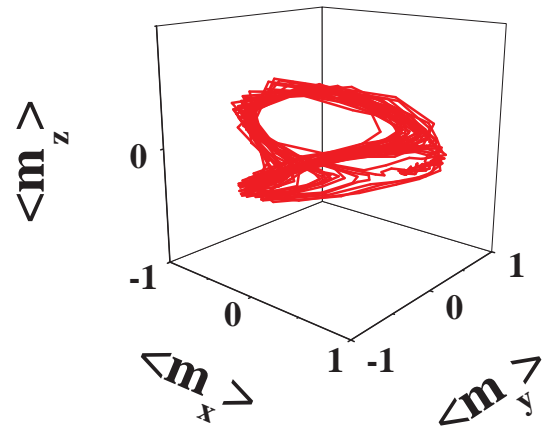

(b)

Fig. 3.14: Visualization of the exchange constant influence on the magnetization dynamics in the etched geometry at $\mathrm{I}=13 \mathrm{~mA}$. Magnetization orbit for (a) $\mathrm{A}=A_{0}$ typical for the region III, and (b) $\mathrm{A}=2.5 A_{0}$ approaching the border between regions III and II

important clues about the importance of the exchange field and therefore $\mathrm{A}=3 A_{0}$ is assumed for further study. Second, as the magnetization always stays inhomogeneous to some extend, the torque calculated locally (cell by cell) inherits this inhomogeneity and we shall scale the torque strength by a factor of 0.5 to counterbalance this effect. Third, micromagnetically the transition from dynamic region I to II was impossible. Thus, in order to observe the OPP, one has to force dynamics in the region II by forcing the transition from region III to II (i.e. imposing the AP initial state). As presented in Fig. 3.15, indeed under all above mentioned assumptions both models converge. Micromagnetic dynamics is forced first in the region III supporting $\mathrm{IPP}_{-}$, then switching towards $\mathrm{P}$ state takes place (range of current where no sustained oscillations are observed, as discussed before), and then IPP branch (region I) is triggered. At certain threshold, however, the dynamics in region II supporting blueshift can be obtained. The threshold of this OPP 
coincides with the experimental one from [18]. The frequency gap associated with the transition IPP-OPP in the macrospin approximation does not fit to the experimental observations, i.e. according to macrospin data at $12 \mathrm{~mA}$ the frequency reaches $11.6 \mathrm{GHz}$ and experimentally frequencies not higher than $4 \mathrm{GHz}$ were reported.

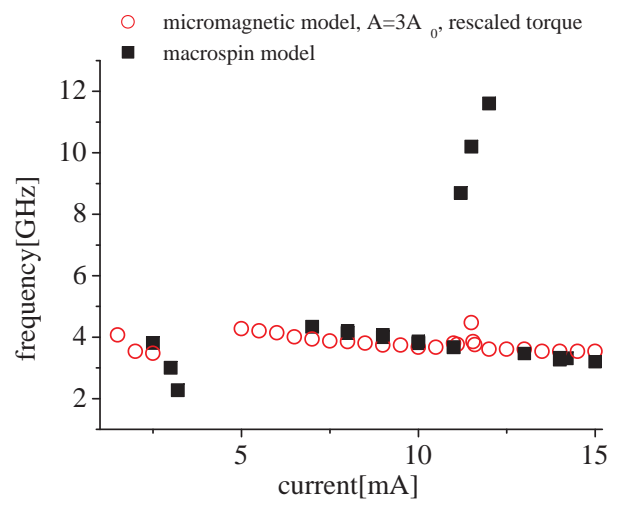

Fig. 3.15: Comparison of the frequency vs. current behavior at $0 \mathrm{mT}$ for the macrospin and micromagnetic models with $\mathrm{A}=3 A_{0}$. As described in the text, in the micromagnetic model the torque strength has been scaled by a factor of 0.5 in order to counterbalance the effect of its inhomogeneous nature. The threshold for OPP predicted here by both models coincides with the experimental one [18]. The magnitude of the frequency jump associated with the transition IPP-OPP in the macrospin approximation does not fit the experimental values and micromagnetic approach proves to be more accurate.

Micromagnetic approach proves to be more accurate, as no such gap is observed and the OPP mode frequency around $4 \mathrm{GHz}$ is in agreement with the measured one. The origin of this discrepancy is linked to the fact that the macrospin precession takes place around the self magnetostatic field axis [31] 
whereas in the real systems there are other contributions to the effective field, mainly the exchange field, which is beyond single domain approximation. Micromagnetic model not only incorporates this factor, but also treating the system as continuum accounts for the inhomogeneous character of the effective field counterbalancing above mentioned effect and thus, no frequency gap is observed in the IPP-OPP transition.

Summarizing, the micromagnetic dynamics in all three regions have been reported. Moreover, the redshift was identified as the IPP - and the $\mathrm{IPP}_{+}$ mode, whereas the blueshift as the OPP mode. Employing Micromagnetic Spectral Mapping Technique (MSMT) [61, 62, 63, 64] the spatial profile of these modes have been investigated. Monitoring the temporal evolution of the magnetization vector field allows, in the frames of MSMT, for determination of the spatial character of each peak in the spectral diagram. As presented in Fig. 3.16a, the main peak in IPP _ spectrum corresponds to the uniform mode. Additionally, a side peak representing another uniform mode is visible together with higher order mode exhibiting hybrid spatial character. The modal analysis of the $\mathrm{IPP}_{+}$is even simpler revealing high power uniform mode and a weak edge mode associated with a minor peak, as shown in Fig. 3.16b. In both cases, the magnetization changes uniformly across the sample, giving rise to one dominant peak.

The spectrum representing the OPP (Fig. 3.16c) is more complicated. One should consider that the magnetization precessing out-of-plane partly looses its coherence [34]. Therefore, even though the main and dominant mode remains uniform, it is preceded by a quasi uniform mode and followed by numerous edge and hybrid modes. In the picture of an evolving magnetization vector field, these modes simply correspond to the rotation being triggered in different parts of the sample. Moreover, at around $12.5 \mathrm{GHz}$ an additional peak is observed. The magnetization in the central region of the sample tends to oscillate uniformly, giving rise to this extra central mode. Even though most of the power is emitted by the predominant uniform mode, 
the appearance of these side modes indicates that additional inhomogeneities, like the Oersted field, could suppress the main mode favoring more complicated modal structures. Still, since the frequency of the predominant mode is hardly influenced by the Oersted field, and its impact on the mode spatial localization is beyond the interest of this section, this contribution was simply neglected.

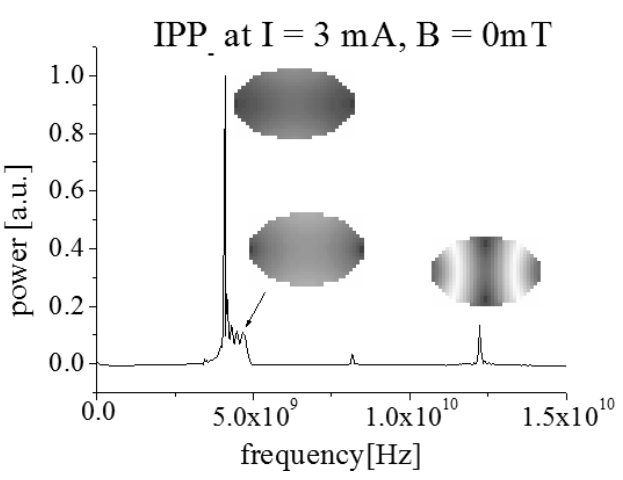

(a)

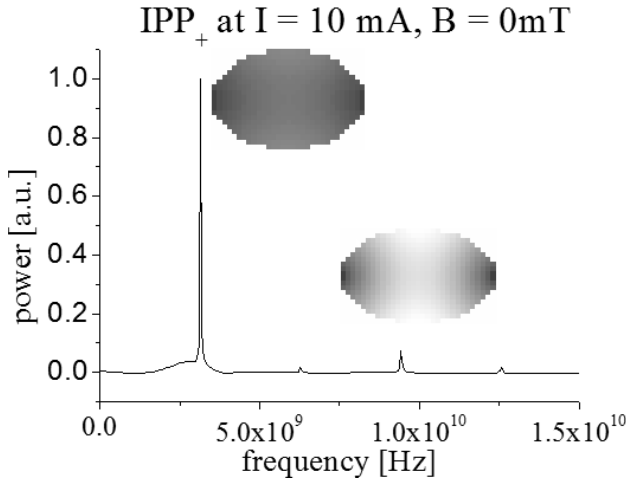

(b)

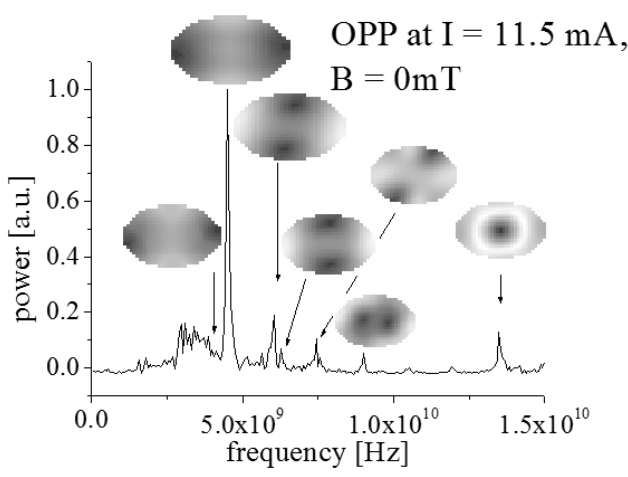

(c)

Fig. 3.16: The spectrum and power density plots for (a) $\mathrm{IPP}_{-}$, (b) $\mathrm{IPP}_{+}$and (c) OPP mode. The main excited mode in all cases is the uniform mode. The IPP modes spectra exhibit some minor higher order edge and hybrid modes, whereas the spectrum of the OPP mode reveals the existence of various additional modes (hybrid, edge and central modes). 
The fact that experimentally the OPP was reported by starting from $\mathrm{P}$ state (opposite to our results) means that transition between the region I and II prohibited micromagnetically is experimentally possible due to thermal activation. As performed simulations neglect the effect of temperature, the dynamic region II can be only reached by transition from region III.

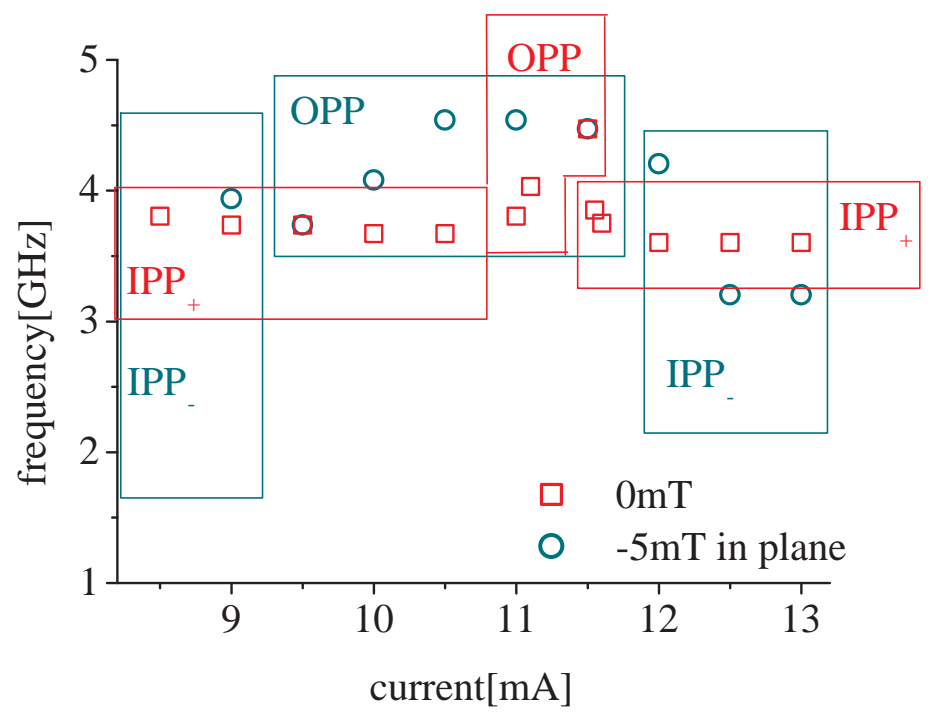

Fig. 3.17: Results of micromagnetic model at zero and low applied field. The dynamic response at $0 \mathrm{mT}$ and $-5 \mathrm{mT}$ applied in-plane. In absence of external field $\mathrm{IPP}_{+}$transition into OPP at $10.5 \mathrm{~mA}$ and back to IPP above $11.5 \mathrm{~mA}$ is observed. At $-5 \mathrm{mT}$ the transitions from IPP - to OPP and back to IPP - are observed at $9.5 \mathrm{~mA}$ and $12 \mathrm{~mA}$ respectively. Both, blueshift linear at $0 \mathrm{mT}$ and nonlinear at $-5 \mathrm{mT}$ predicted micromagnetically, are consistent with the experimental results [18]. Macrospin ceases to show the saturation regime observed experimentally at $-5 \mathrm{mT}$ but full micromagnetic study correctly predict this feature.

One should note that not all regimes (dynamics in all regions I, II, III) predicted by the simulations for the extended geometry were observed ex- 
perimentally. Low angle IPP does not provide enough output power to be measured via GMR effect. Therefore, in order to conduct meaningful comparison we have concentrated on the OPP regime (region II), which was both predicted numerically and observed experimentally. In the absence of external field a satisfactory qualitative agreement has been reached (Fig. 3.17, compare to Fig. 6a in Ref. [18]). Not only the threshold current $\left(I_{t h, s i m}=11 \mathrm{~mA}\right.$ compared to $\left.I_{t h, e x p}=10 \mathrm{~mA}\right)$, but also the agility (0.6 GHz/mA and $0.7 \mathrm{GHz} / \mathrm{mA}$ respectively) are in good agreement. The remaining quantitative difference in frequency values is a consequence of uncertain estimation of the factors entering micromagnetic model, like saturation magnetization and/or damping.

Moreover, the dynamics at $-5 \mathrm{mT}$ IP field reveals that OPP threshold current is smaller with respect to $0 \mathrm{mT}$ case, which is again consistent with the experimental results. Interestingly, both approximately linear at $0 \mathrm{mT}$ and nonlinear at $-5 \mathrm{mT}$ behavior of the frequency as a function of current, are well reproduced in frames of micromagnetic model. Note that this feature was not reported within single domain approximation. Furthermore, micromagnetic model predicts experimentally observed saturation at $-5 \mathrm{mT}$, i.e. in a certain current range the frequency stays relatively constant. In the model it is associated with the large angle orbit stabilization around the torque minimum, i.e. the system approaches the border of dynamic region II and III, and the torque shape becomes flat around its minimum. Current increase leads to transition between OPP (region II) and IPP_ (region III) and reappearance of the clamshell orbit. However, since neither was the IPP reported in the experiment prior to the appearance of OPP, as predicted micromagnetically, nor it could have been detected following OPP regime (because of low output power) so obviously the experimental cut off current refers to the threshold current for IPP reappearance in the model. Note that micromagnetically the main mode (supported over largest range of currents) in case of $0 \mathrm{mT}$ was the $\mathrm{IPP}_{+}$, whereas even low applied field of $-5 \mathrm{mT}$ 
IP forced the dynamics in region III and, therefore, the IPP _ was observed as the main mode. In other words direct dynamics in region II in absence of external field (and associated linear frequency vs. current slope) is favored by sufficiently high exchange field and additional external field forced the transition from region III to region II resulting in the appearance of the saturation regime. Clearly, the OPP in both cases is preceded by different dynamics.

\subsection{Conclusion}

This chapter was focussed on description of the wavy torque driven dynamics observed in pillar geometry. The existence of field-assisted OPP regime was confirmed only within the macrospin model [31]. Assuming that the fixed layer is etched giving rise to ICF, the OPP modes can be triggered in absence of any additional external field. Still, even moderate external fields (90 mT) did not support the OPP mode within the micromagnetic study. In turn, the IPP _ mode has been observed, indicating that the dynamics in the region of the torque diagram where the macrospin OPP mode was observed is not supported micromagnetically at all. This discrepancy leads to the conclusion that - from the point of view of micromagnetics - more important for the appearance of the OPP mode is the influence of finite exchange field and inhomogeneities of the demagnetizing field than the external field.

Hence, by setting proper initial state and the exchange constant favoring the appearance of the OPP, a good qualitative agreement was reached between the predictions of both models. Thus, only full micromagnetic model has predicted correctly dynamics reported experimentally i.e. quantitatively the frequency values as well as qualitative features like the nonlinear frequency blueshift with current and the appearance of saturation regime at low applied field [32]. 


\section{VORTEX OSCILLATIONS IN SINGLE POINT CONTACT GEOMETRY}

In this chapter the investigation of magnetization dynamics in point contact geometry is presented along with the explanation of computational difficulties involved. Particular case of a single magnetic vortex based Spin Transfer Nano Oscillator (STNO) is discussed. This study has been carried out in collaboration with the experimental group CNRS/Thales, Palaiseau, France [65]. The sample under study, owing to a novel fabrication technique, exhibits specific locally constrained geometry. Hereafter its impact on the overall behavior of the system is studied. Intriguing dependencies are discovered leading to the qualitative understanding of measured trends and quantitative match with numeric results.

\subsection{Introduction to vortex mode}

In most of the state- of-the-art spintronics experiments high fields were required to observe sustained output $[66,26]$. First spin torque oscillators were based on the excitation of the quasi-uniform precessional mode. Only very lately it has been demonstrated that spin torque can also drive a magnetic vortex into gyration. Model vortex configuration is characterized as following. Most of the sample stays magnetized IP and only in the central region the OP component is observed. The latter, called also the core forms as a compromise between the magnetostatic field - trying to keep the mag-

netization IP and the exchange field assuring continuous changes between 
neighboring spins. This way magnetic vortex is characterized by its chirality and polarity. The first one defines the IP direction of the magnetization curling and is either clockwise (cw) or counterclockwise (ccw). The second defines the OP core orientation. An example of such a structure with ccw chirality and positive polarity is presented in Fig. 4.1.

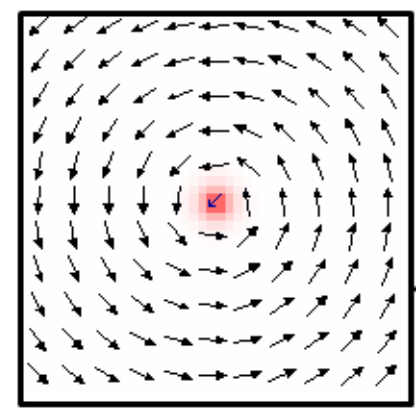

Fig. 4.1: An example of a magnetic vortex of ccw chirality and positive polarity. OP magnetization component in color scale.

Sustained oscillations of magnetic vortex have been observed both in pillar $[38,43]$ and PC geometry [37, 39]. Very recently it has also been demonstrated that such vortex based PC STNO might work in absence of external field [19]. Therefore, it became very interesting not only from fundamental point of view but also as a possible functional device. In particular, sub$\mathrm{MHz}$ linewidths and large GMR output signal are promising for the future applications.

For geometry reasons vortex oscillations a pillar and in a PC structure should be treated separately. In the first case, due to lateral confinement, a displaced vortex induces magnetic charges at the lateral surfaces. These result in the appearance of the dipolar stray field and, therefore, vortex energy becomes position dependent. Hence, the oscillations correspond to the gyration of the core around the centered equilibrium position. In PC device, however, lateral confinement, at least in principle (in absence of pinning centers etc.), does not exist. If discounting any possible impurities and defects 
in the sample the vortex energy is position independent. However, when current is passed the associated Oersted field creates an attractive potential defining the center of the sample as lowest energy position for the vortex core. Oscillations are observed when the dissipation term is counterbalanced by the ST resulting in vortex orbiting the contact.

Even though basic mechanism and the general features of this mode are well understood there are aspects yet to be clarified. In particular, the rigid vortex model was successfully applied for explanation of frequency blueshift with current and redshift with field, as reported experimentally in metallic exchange-biased PC spin valves [39]. This model, however, failed to predict and justify the existence of the threshold and cut-off currents. Moreover, reports of numerical studies of spin current induced dynamics of the vortex mode are rather seldom in the literature. This chapter adds some important insight along these lines.

\subsection{Point contact geometry, computational issues}

The reason why PC devices are poorly studied numerically is twofold. First of all, the simulations of a laterally extended system as such encounter many methodological difficulties since only a finite structure can be treated computationally. If already attempted, the simulations of large structures are very time taking and, therefore, only deterministic study is carried out in most of the cases i.e. thermal fields are neglected for sake of simplicity. Even though it is well known that modes, in particular vortex mode, might be also temperature assisted [43] in the following this contribution is neglected. Second, if there is energy carried toward the boundary of the computational area, as it is the case of the spin waves, pure numeric reflection artifact appears and influences the final frequency response. In real systems the energy is dissipated within the sample before it reaches the boundary of the micron size device. However, in the simulations, where the computational area is 
limited to hundreds of nanometers and no defects are accounted for the spin wave reflects and interferes with the incoming wave. This problem has been addressed in Ref. [67] by introducing Absorbing Boundary Conditions (ABS) i.e. space dependent damping assuring that energy dissipates before the wave reaches computational boundary. This approach shall be presented shortly below.

\subsubsection{Linear mode}

Investigating propagating mode one aims at understanding the free layer dynamics driven by the combined effect of the applied OP field and the STT.

Simulation of an actual lateral size of the system $\sim 10-20 \mu \mathrm{m}$ would lead to prohibitively long computational times but on the other hand simulation of a confined system $\sim 1 \mu \mathrm{m}$, that is still much larger than the PC radius, introduces the problem of proper boundary conditions and wave reflection. In order to minimize this reflection a space-dependent dissipation has been introduced. The idea is to define spatial radial increase of damping parameter, so that close to the $\mathrm{PC}$ the damping parameter is given its physical value and far from center its value is artificially increased. The abrupt and the smooth dissipation profile have been considered together with their impact on the computed frequency.

The computational area was discretized into $4 \times 4 \times 5 \mathrm{~nm}^{3}$ cells. Following parameters: the exchange constant $\mathrm{A}=1.3 \cdot 10^{-11} \mathrm{~J} / \mathrm{m}$, damping $\alpha=0.015$, saturation magnetization $\mathrm{M}_{\mathrm{s}}=500 \cdot 10^{3} \mathrm{~A} / \mathrm{m}$ and no crystalline anisotropy for free layer and saturation magnetization $\mathrm{P}_{\mathrm{s}}=1.5 \cdot 10^{6} \mathrm{~A} / \mathrm{m}$, cubic anisotropy constant $\mathrm{K}_{\mathrm{ani}}=5.6 \cdot 10^{4} \mathrm{~J} / \mathrm{m}^{3}$ for the fixed layer were used. A uniform current distribution was considered.

Consider metallic multilayer with the $\mathrm{ST}$ valve consisting of $\mathrm{CoFe} / \mathrm{Cu} / \mathrm{Py}$ as presented in Fig. 4.2. The thick layer (called polarizer hereafter) is assumed to be static in time and only the dynamics of the active thin Py 
layer (called free layer hereafter) is resolved. The field applied along the OP direction tilts the magnetization in the polarizer $30^{\circ} \mathrm{OP}$. First the initial configuration (without ST) in the active layer is found by allowing the system relax to the equilibrium position. The magnetostatic coupling from the fixed layer is calculated but only its OP component is accounted for in the dynamic study. The Oersted field and thermal field are neglected. ST is employed in from proposed in Ref. [6].

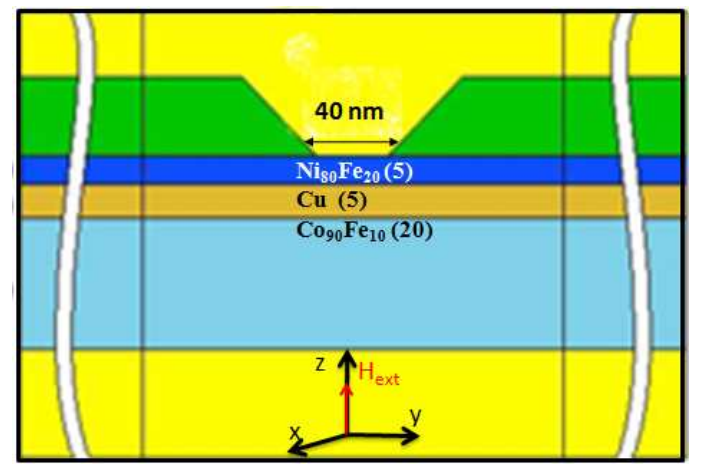

Fig. 4.2: Geometry of a metallic point contact for the study of spin waves.

Following is observed. The spin wave that is excited below the PC area propagates across the sample and reflects form the boundaries. These reflections are visible in inset (b) Fig. 4.4 where the incoming and reflected waves interact resulting in a complicated magnetization pattern. In order to minimize these reflections the model proposed by Berkov and Gorn [67] is employed. The model assumes that inside the PC the dissipation is given its physical value but far from it is varied in a smooth way to avoid reflections. An example of such profile of the dissipation parameter is presented in black in inset (a) Fig. 4.4. Different profiles of spatially varying dissipation have been investigated systematically in order to define its optimal shape. It is worth emphasizing that the distance where the dissipation value starts 
varying should be chosen carefully, if this point is fixed either too close to the boundary or too close to the PC the computed frequency is be affected. Introduction of such dissipation profile in the computational area equals the implementation of the ABC. The results of this approach are shown in inset (c) Fig. 4.4. The spin waves nucleated under the PC propagate across the sample dissipating energy on the way so that they barely reach boundary and no reflections are observed.

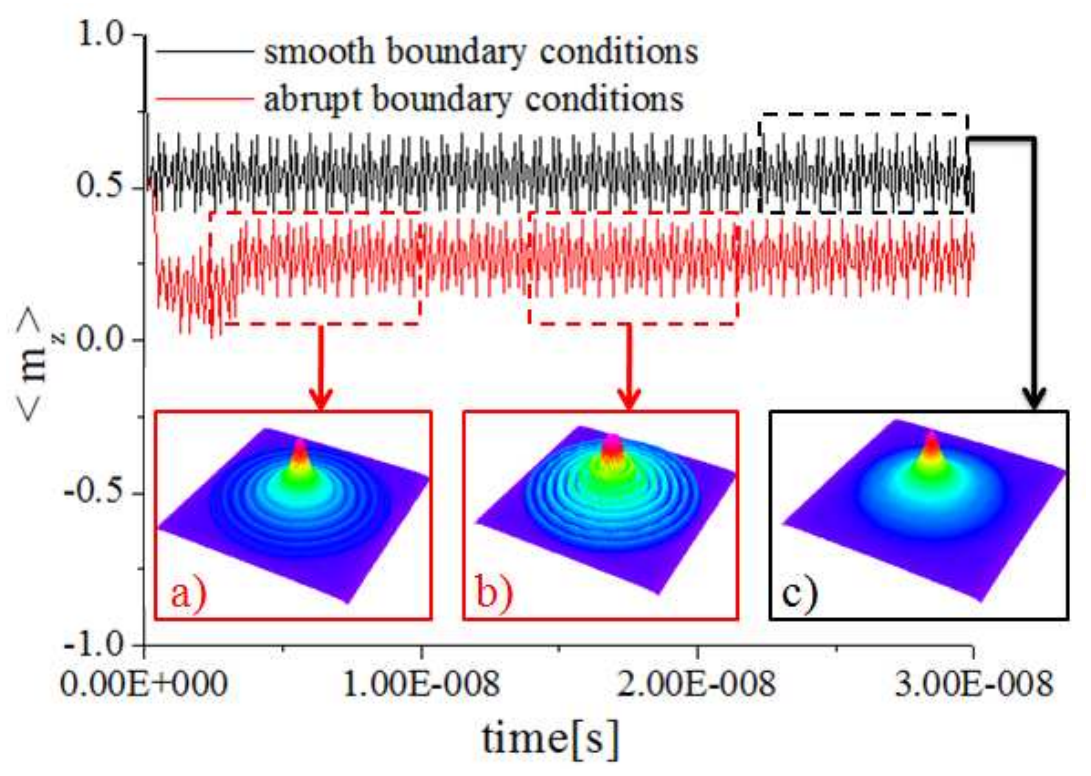

Fig. 4.3: Temporal evolution of the average $\mathrm{m}_{\mathrm{z}}$ component below the PC. Insets represent power spectra as extracted from marked time windows when abrupt (a,b) and smooth (c) ABC considered.

The abrupt change of dissipation profile, as proposed in Ref. [68], has also been investigated. In this approach inside certain circular area the dissipation is assigned its physical value and outside it abruptly changes by two orders of magnitude, as presented with red line in inset (a) Fig. 4.4. It turns 
out that the abrupt dissipation profile ceases to work properly, as predicted in Ref. [67], since any abrupt change is the material parameters (damping in this case) leads to wave reflection. Monitoring the time evolution of the averaged magnetization $\mathrm{z}-$-component below the $\mathrm{PC}$ and comparing it for the case of smooth and abrupt $\mathrm{ABC}$ one observes some differences. As presented in Fig. 4.3, if abrupt $\mathrm{ABC}$ are employed at some point in time the first spin wave reaches the boundary and reflects from it which can be seen as trajectory change in the time evolution of the averaged $\mathrm{z}$-component of the magnetization. Whereas, if the smooth $\mathrm{ABC}$ are implemented the magnetization reaches its equilibrium steady precession state without change of trajectory.

In order to visualize what is happening in the sample one should have a look at power density plots. Employing abrupt ABC and averaging the power over some early stage time window (inset (a)) Fig. 4.3) one observes the interference pattern building up. The magnitude of this interference grows as the power is averaged over later time window (inset (b) Fig. 4.3). On the contrary, no interference is reported when smooth $\mathrm{ABC}$ are employed (inset (c) Fig. 4.3).

To complete the comparison between this two approaches frequency spectra are computed to compare the linewidths. As presented in Fig. 4.4 the linewidth emerging from simulations where abrupt boundary conditions were employed is around three times larger than in case of simulations with smooth ABC implemented (180 MHz and $65 \mathrm{MHz}$, respectively). It is worth reminding at this point that all simulations were performed neglecting the effect of thermal fluctuations so obviously the broadening of the linewidth can only result from the wave reflections and with it associated interferences. 


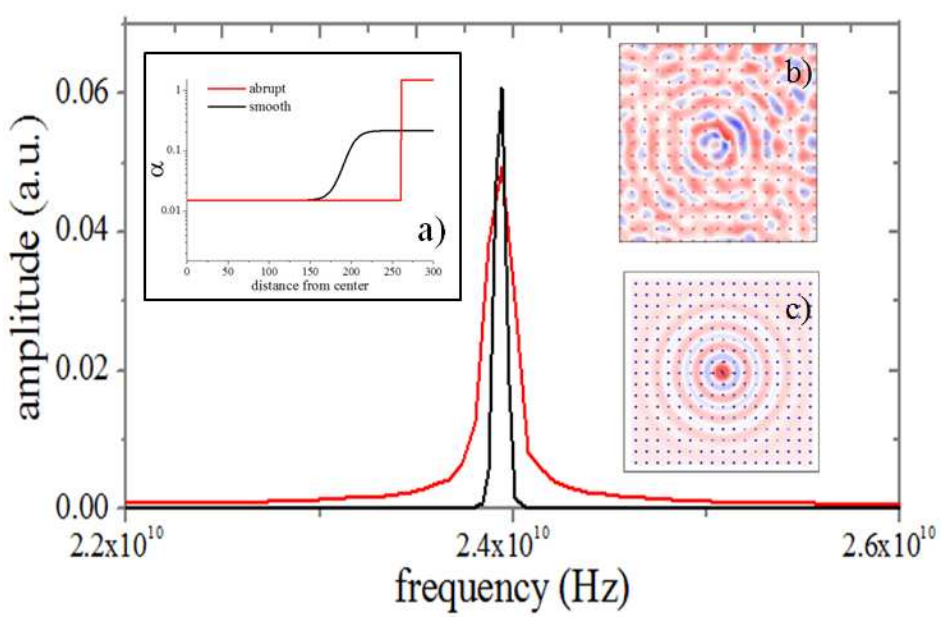

Fig. 4.4: Frequency as extracted from simulations with abrupt (red) and smooth (black) ABC with the profile as presented in the inset. Abrupt ABC lead to broadening of the linewidth.

\subsubsection{Vortex mode}

In case of magnetic vortex in PC geometry no energy is carried toward the boundaries so, at least in principle, no $\mathrm{ABC}$ implementation is required. Still, there are other methodological problems to be addressed. In pillar geometry it is the lateral confinement that gives rise to lateral side charges and the appearance of the dipolar stray field. It creates space-dependent potential and in presence of SPC vortex gyration can be observed. Such lateral confinement leads to the appearance of volume and surface charges that determine the vortex precession frequency as has been demonstrated in Refs. [40, 69, 70, 71] for the case magnetic dots in absence of currents.

In a real PC device, considering micron size of the sample, the absence of above mentioned confinement leads to the negligible effect of the lateral charges. However, in a numerically modeled vortex PC system due to computational size and/or time limitations the existence of side charges is unavoidable, i.e. due to finite meshing some additional magnetic charges at the 
sides of the computational area are always present. Moreover, as soon as the vortex moves away from its equilibrium position the profile of the magnetostatic energy created by the charges becomes vortex position dependent (and, therefore, also time dependent).

The reader should be aware that this subject is rather complex and has never been studied systematically, there exist no relevant literature. The correct way to proceed requires the estimation of the magnetostatic charges created by the moving vortex and their neutralization by adding opposite charges. This task is highly complicated and has not been attempted so far. In the following different approach is employed. A systematic study of vortex gyration frequency on the size of the computational area is carried out in order to estimate the size above which the effect of side magnetic charges seems to be negligible.

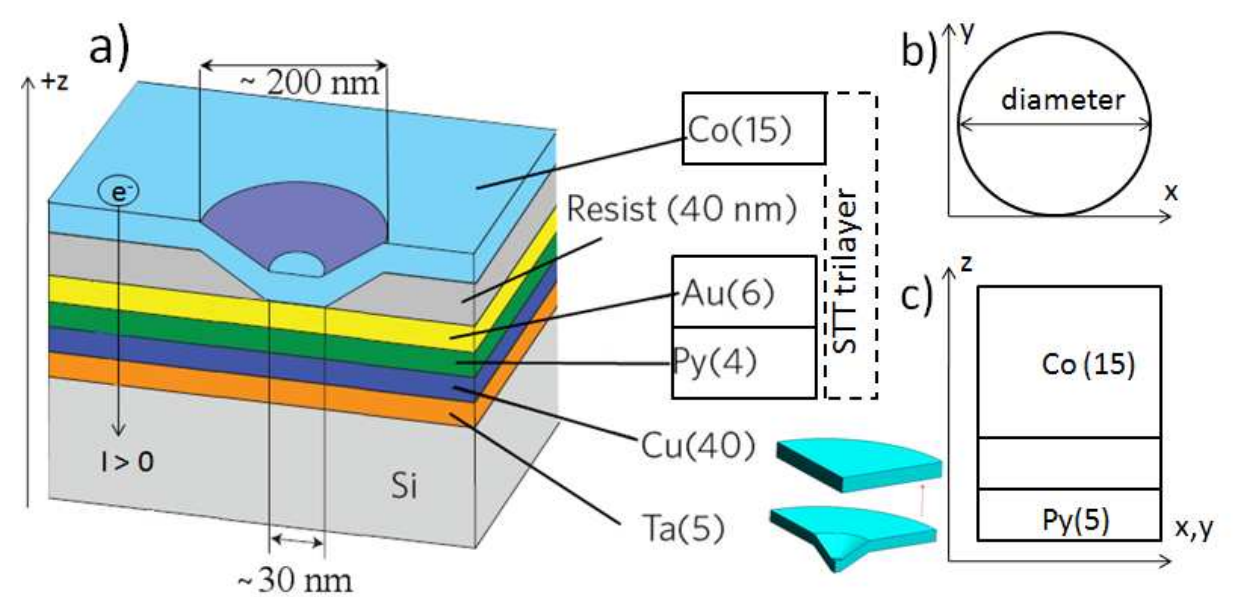

Fig. 4.5: Experimental spin valve structure with highlighted STT trilayer (a). Consider non-flat profile of the Co layer. In-plane circular cross-section of model flat trilayer for computational issues study (b) and its side view with schematically shown the transformation of the real V-shape Co layer into flat model (c). Diameter refers to the computational area. 
The experimental spin valve structure, which serves as a model structure, is presented in Fig. 4.5a where the STT trilayer is highlighted. Technical details about the fabrication process and other sample characteristics are to be found in the following sections. For the study of computational issues concerning vortex gyration mode the structure is simplified i.e. only the main features of the geometry are incorporated into the model. Thus, a circular cross-section, as shown in Fig. 4.5b, and the side view, as in Fig. 4.5c, are assumed. Experimentally, the oscillations were observed at positive current defined by the electrons flowing from Co to Py layer. The definition of the active layer and the polarizer in this structure is a separate task and shall be treated in the later sections. Focusing at computational issues first the magnetization in Co layer is allowed to evolve in time and Py layer serves as the reference in-plane static polarizer. Then the frequency response at different diameters of the computational area is studied. Second, Py is assumed the active free layer and Co the fixed layer and analogous study is carried out. Only the dynamics of the active layer is resolved in both cases assuming a centered vortex for the initial magnetization configuration.

Results are presented in Fig. 4.6. Considering dynamics in Co layer (Fig. 4.6a) one observes that as the diameter of the computational area is increased the frequency decreases. Surprisingly, this behavior seem not to be linear with current indicating complicated origin of the tendency as such. Moreover, the frequency decreases very fast up to the diameter of $1500 \mathrm{~nm}$ and is almost constant above this size. Similar behavior is reported when Py is set the active layer in the system (Fig. 4.6b).

The problem itself is complex and, therefore, based on the above result a diameter of $2500 \mathrm{~nm}$ is assumed for the study presented in the next paragraph. As demonstrated, independent of the definition of the free layer the effect of lateral charges on the frequency of the oscillation vortex is negligible at this computational size. 
As long as ST is neglected and the mesh cell is shorter that the exchange length one should approximate the dynamic evolution in a correct way. However, the introduction of STT involves some further complications. Considering the interfacial character of STT [72], severe constraints on the mesh size in the $\mathrm{z}$-direction should be observed. This introduces methodological problem, particularly when considering thick layers leading to prohibitively long computational times. On the other hand, the layer thickness was the historically first argument to disregard the dynamics [6] within it. Though, in the experimental design considered the ST effect is expected to be stronger in the $5 \mathrm{~nm}$ thick Py layer than in $15 \mathrm{~nm}$ thick Co layer favoring the STT-driven dynamics in Py and leading to lower overestimation of the real frequency. Hence, in absence of exchange-bias the Co layer can not be, at least in principle, defined fixed in time a priori.
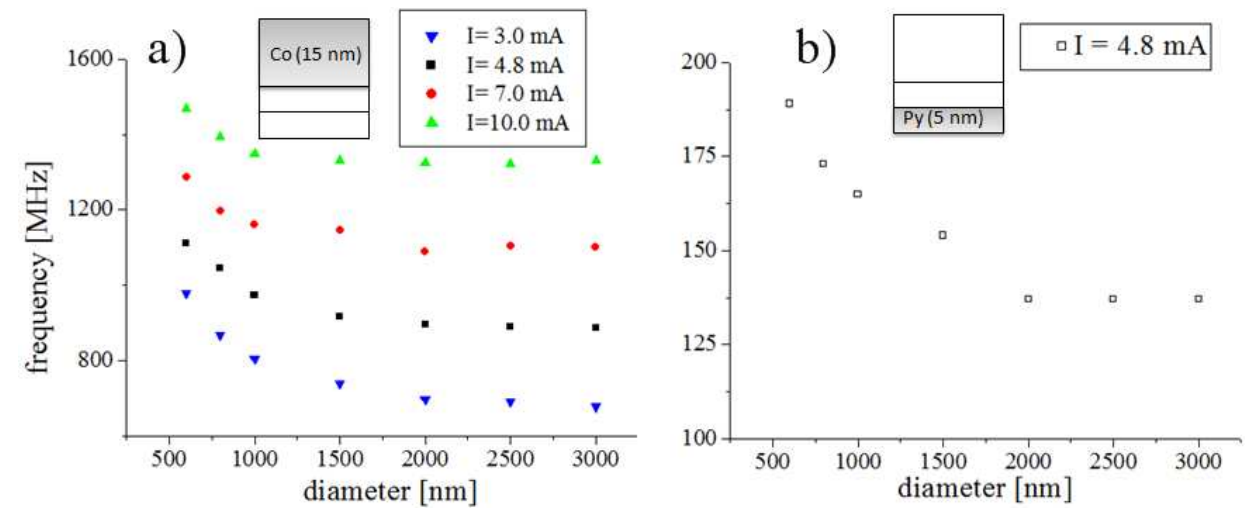

Fig. 4.6: Frequency dependence on the size of the computational area at different currents when only dynamics in Co (a) or (b) Py layer is resolved as specified in the insets.

Therefore, in the following different meshing in the $\mathrm{z}$-direction is applied for the $15 \mathrm{~nm}$ thick Co layer resulting in one, two and three computational 
sublayers, respectively. The frequency of the gyrating vortex in each case is presented in Fig. 4.7 indicating that finer meshing leads to lower frequency. It is attributed to the fact that when only one mesh layer is allowed in $\mathrm{z}^{-}$ direction the internal vortex structure can not change and can be treated within rigid limit. With finer mesh, the core is subject to deformation (inset Fig. 4.7) which minimizes the internal energy and, therefore, leads to lower frequency.

To conclude, one should remember that the frequency obtained numerically is always an overestimation with respect to the one measured in the real device in particular when treating with thick layers. Above studied magnetostatic and exchange energy contributions have been demonstrated to play important role in the vortex core deformation profile. The question of the implementation of the superficial character of the ST effect stays open.

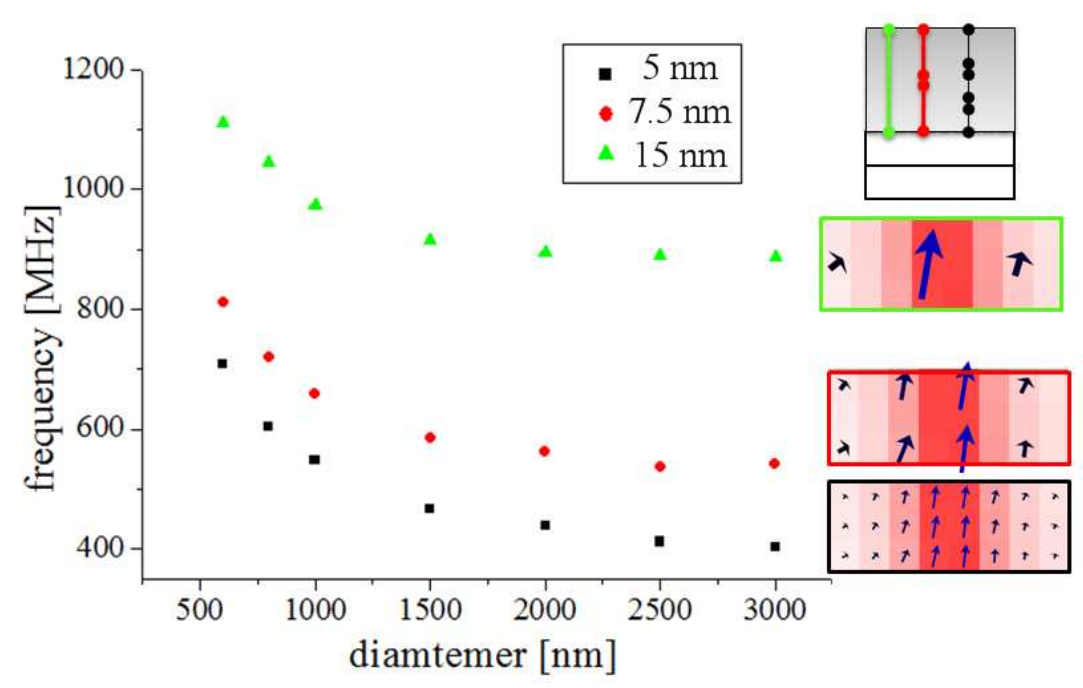

Fig. 4.7: Frequency dependence on the mesh in the $\mathrm{z}$-direction in the Co layer. $5 \mathrm{~nm}, 7.5 \mathrm{~nm}$ and $15 \mathrm{~nm}$ are considered corresponding to 1, 2 or 3 mesh layers as schematically shown in the inset. 


\subsection{Initial configuration in hybrid geometry}

Above discussed computational issues revealed some interesting features of the vortex mode. However, flat profiles of the layers were assumed, which is significant simplification of the real sample. Hence, after having highlighted the methodological problems in general now the attention is to be focussed on the geometry induced features in the device as in Fig. 4.5a. The constriction in contact vicinity Co layer shall have influence on the current and with it associated Oersted field distribution. Note that current and ST effect on one side and attractive potential created by the Oersted field on the other side are the crucial factors determining the dynamics of the vortex mode. Therefore, calculation of their distributions is essential to further compare numerical result with the experimentally reported trends.

\subsubsection{Sample characteristics and simulation parameters}

The sample under consideration is a sputtered multilayer of $5 \mathrm{~nm}$ Ta / $40 \mathrm{~nm} \mathrm{Cu} / 4 \mathrm{~nm} \mathrm{Py} \mathrm{/} 6 \mathrm{~nm} \mathrm{Au} \mathrm{/} 15 \mathrm{~nm}$ Co / $100 \mathrm{~nm} \mathrm{Au}$. The contact of $20 \mathrm{~nm}$ nominal diameter was opened by conductive tip atomic microscope nano-indentation and plasma etch [47]. As a result a locally constrained (in vicinity of the contact) Co layer is obtained. Further details of the fabrication methods are to be found in Ref. [14]. The structure is presented in Fig. 4.5a, where $\mathrm{Py} / \mathrm{Au} / \mathrm{Co}$ represents the relevant STT trilayer. In the experimental data, presented in the next paragraphs, the oscillations were measured for the electrons flowing from Co to Py layer, corresponding to positive current in the convention adapted. Before applying the current and acquisition of each spectrum a large reset field saturates the sample.

Micromagnetic simulations were performed with public code OOMMF [73]. Following parameters were used: saturation magnetization and exchange constant for Co and Py $1.4 \cdot 10^{6} \mathrm{~A} / \mathrm{m}, 3.0 \cdot 10^{-11} \mathrm{~J} / \mathrm{m}$ and $0.6 \cdot 10^{6} \mathrm{~A} / \mathrm{m}$, $1.3 \cdot 10^{-11} \mathrm{~J} / \mathrm{m}$, respectively. For the purpose of static study (without $\mathrm{ST}$ ) 
the computational region of $2500 \times 2500 \times 65 \mathrm{~nm}^{3}$ discretized in $5 \times 5 \times 2.5 \mathrm{~nm}^{3}$ cells is considered. The current-driven dynamics is resolved only in the Py layer represented computationally as $2500 \times 2500 \times 4 \mathrm{~nm}^{3}$ region of circular cross-section and discretized into $5 \times 5 \times 4 \mathrm{~nm}^{3}$ cells. Following values were used for the damping constant and polarizing factor $\alpha=0.009, P=0.1$, respectively. In the simulations the contact radius is assumed to be $15 \mathrm{~nm}$. Thermal fluctuations have been neglected.

\subsubsection{Geometry induced aspects}

Considering sample geometry, even in absence of circularly symmetric current associated Oersted field, such symmetry is imposed by the cone-like constricted PC in Co layer. Simulation show that already the sole effect of the demagnetizing field, in this locally confined structure, favors vortex formation. Previous works [74] demonstrate that pure magnetostatic considerations of a coupled system of constrained disks yield the result of two vortices of different chirality nucleated one in each layer. On the other hand, presence of the cone-like PC profile in the Co layer from geometric point of view introduces lateral constrictions. These, by means of magnetostatic interactions, are indirectly translated also into the Py layer.

Indeed, static simulations (without current) verify that the nucleation of two vortices of opposite chirality, as presented in Fig. 4.8, defines the sample minimum energy configuration. Several simulations have been carried out changing the shape (circle, square) and the size of the computational area and the same configuration was reached in all cases.

Assume now that the SPC is applied while Py is the active layer and Co layer is the static in time polarizer. Thus, the magnetization temporal evolution is to be resolved only in Py layer whose initial configuration is represented in Fig. 4.8b. On the other hand Co layer, as in Fig. 4.8c, is taken as the polarizing profile for the electrons. Hence, when positive current is applied the configuration in Py layer is expected to be destabilized from the 
incoming polarized electrons, which is in agreement with the numeric results (not shown). One observes vortex gyration but the reported frequency is almost an order of magnitude larger than the frequency observed experimentally.

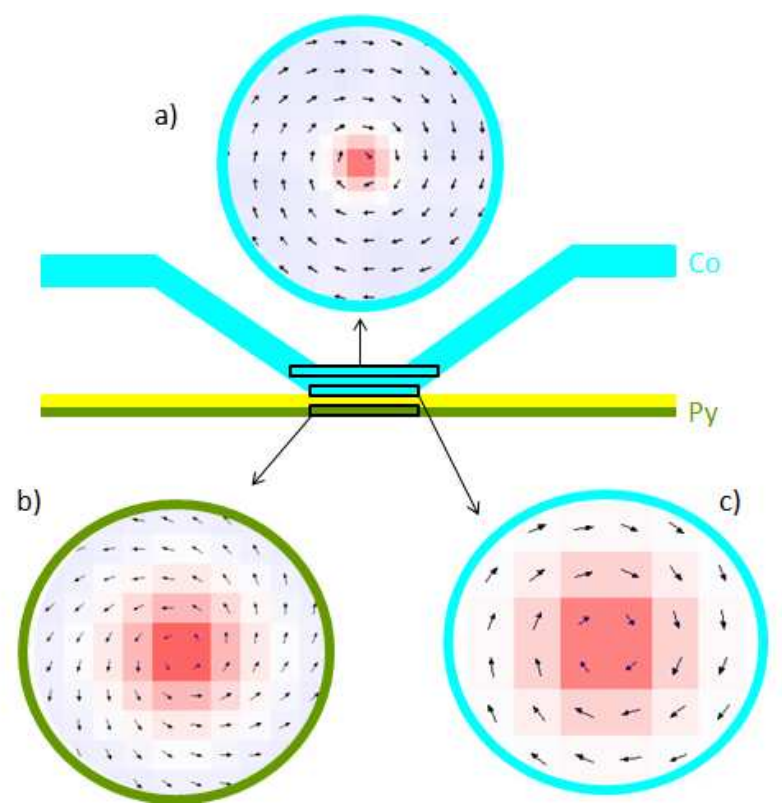

Fig. 4.8: The configuration in Co layer on the top of the PC (a), and at the PC (c), and in Py layer (b) in absence of current.

Moreover reported trends, exclude the possibility of the existence of two vortices in the system. The MR signal indicates quasi-uniform parallel configuration of both layers. Therefore, the vortex is believed not to be nucleated prior to the current application i.e. nucleation process is driven by the current associated Oersted field which is present first when current is passed. Hence, the exact current and Oersted field distributions are to be calculated and used hereafter in order to investigate their role in the nucleation processes and resulting lowest energy magnetic configuration in the sample. 


\subsubsection{Current and Oersted field calculations}

The calculation of the current and associated Oersted field distributions have been performed with the finite element commercial software MagNet [75]. The entire structure including the electrodes is accounted for. Resistivity values of the materials involved that have been used for this calculations are presented in the table below.

$$
\begin{aligned}
& \text { Tab. 4.1: Resistivity of materials, }\left[10^{-8} \Omega \mathrm{m}\right] \text {. } \\
& \begin{array}{cccc}
\hline \hline \mathrm{Au} & \mathrm{Cu} & \mathrm{Py} & \mathrm{Co} \\
\hline 2.5 & 1.7 & 7.0 & 7.0
\end{array}
\end{aligned}
$$

Consider first the spatial distribution of current, Fig. 4.9, when $1 \mathrm{~mA}$ is passed between the lateral top electrode and side surface bottom electrode. The presence of the inclination in Co layer profile forces the current to flow in the plane of the inclined constriction. Thus, through the nanocontact the current flows mostly perpendicular to the plane but in the inclined region in Co layer significant IP currents are present. The density plot at the Co cross-section, inset (a) of Fig. 4.9, reveals that significantly larger current density is found at the edges rather than in the central PC region. On the other hand, the distribution becomes more uniform below the contact in Py layer, inset (b) of Fig. 4.9.

From the current distribution one can calculate the exact profile of the current associated Oersted field. The main graph in Fig. 4.10 presents this profile across chosen cross-sections of the sample together with the analytical profile (at the current of $1 \mathrm{~mA}$ ). These cross sections are marked in the inset where the strength of the field is presented in color. The analytical solution shows its maximum at the edge of the point contact $\left(\mathrm{R}_{\mathrm{PC}}\right)$ and then it decays with the distance (r) as $1 / \mathrm{r}$. As compared to the calculated profiles inside the sample one observes that realistic maximum of the Oersted field is smaller than the analytical prediction and it is shifted outside the PC indicating 
current spreading close to the contact area.

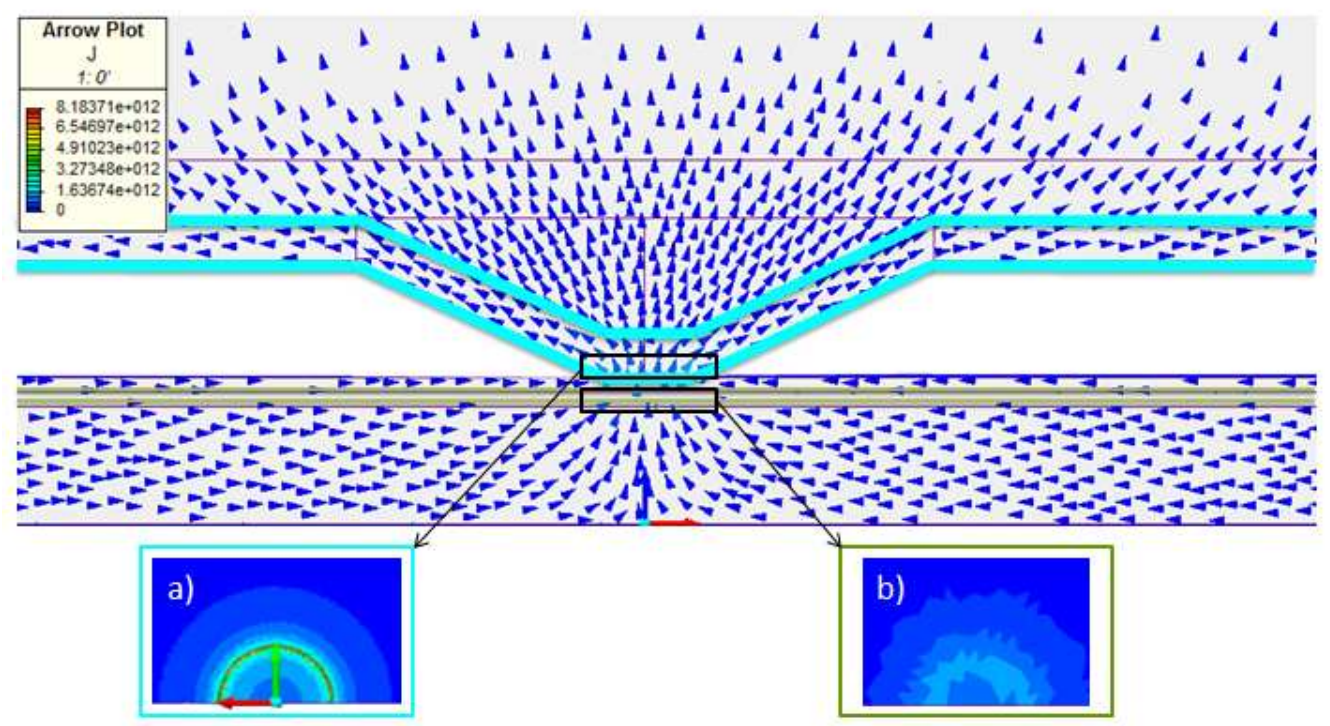

Fig. 4.9: Arrow plot of current distribution throughout the sample at $1 \mathrm{~mA}$. The density plots in vicinity of $\mathrm{PC}$ in Co and Py layer cross-sections, inset (a) and (b), respectively.

Additionally, one should consider that on the top of the Co layer which corresponds to its flat part (blue inverted triangles) the effect of the Oersted field is much weaker than at the contact itself (red circles). Already in the top part of the PC (green triangles) the maximum Oersted field strength is approximately half the value directly at the contact. These are the consequences of the nonuniform current distribution across the sample. In particular, the presence of above mentioned IP currents decreases the maximum of the final profile owing the cancellation effect from opposite IP components in the constriction. 


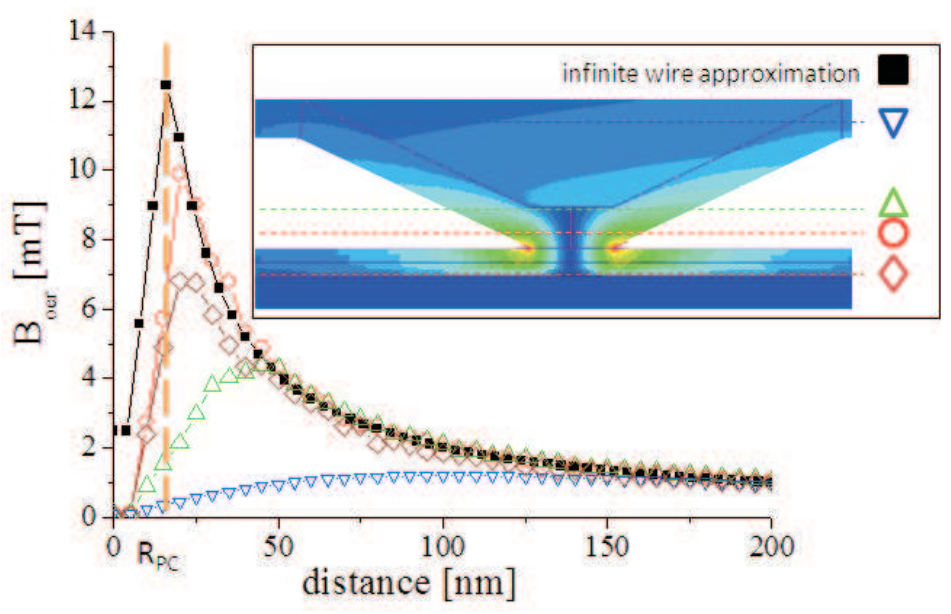

Fig. 4.10: The Oersted field in the sample as calculated with MagNet software [75]. Main figure: profile in the Py layer (purple diamonds), bottom of the Co layer (red circles), top of the contact area (green up triangles) and the flat part of the Co layer (blue down triangles). The analytical profile as calculated from the infinite conductive wire approximation is also shown for comparison (full black squares). Inset presents in color scale the strength of the field additionally marking the positions corresponding to the profiles from the graph. The radius of the point contact $R_{P C}$ is marked with dashed line in the main graph.

\subsubsection{Initial state in presence of currents}

The above results are now accounted for in the determination of the the equilibrium state in absence of ST. Thus, in Py layer, in presence of positive current of $10 \mathrm{~mA}$ a counterclockwise (ccw) vortex is formed following the chirality of the associated Oersted field. Second vortex is formed in Co layer, though of different chirality. Such situation may arise because of the magnetostatic reasons indicating that at the current considered for the nucleation $(10 \mathrm{~mA})$ the demagnetizing field effect is stronger than the Oersted field effect. Consider that qualitatively similar result (two vortices of opposite chirality) was observed when no current was applied, i.e. in absence of 
Oersted field.

The appearance of clockwise (cw) curled vortex in Co is then followed by its expulsion from the PC because in presence of ccw Oersted field the center of the PC is no longer lowest energy position for the cw vortex. The final state is represented by a nonuniform IP magnetization configuration with significant OP components (arising from geometry) inside the PC in Co layer which, when current is passed, serves as a polarizer for the electrons, Fig. 4.11a and c. On the other hand, a ccw vortex configuration is found in Py as presented in Fig. 4.11b.

In the real device there are different possible scenarios with respect to the final state of cw vortex in Co layer. It could either be pinned or annihilated on a defect in the flat part of the layer above the resist. Considering that the Oersted field strength responsible for winding the magnetization is significantly reduced there and that at the edges of a real device the magnetization is mostly IP these predictions seems highly probable but, due to size limitations of the simulated area, can not be verified numerically. However, such scenario is compatible with the experimental indication that there exist only one vortex in the vicinity of the $\mathrm{PC}$ since as soon as the vortex in Co layer moves to the upper flat part of the layer it does not affect the dynamic behavior of the system. If both vortices were present inside the PC the GMR signal would indicate it. Note also that the nonuniform configuration in the Co layer in the PC could be obtained in a different way i.e. not necessarily by the expulsion of cw vortex but as a result of other complicated dynamic processes. Thus, the dynamic evolution predicted by this simulation, which did not include the effect of ST, does not have to coincide with the processes taking place in the real system when the current is applied. Certainly, the mechanisms for vortex nucleation in nanocontact devices are complex [76] and their study is beyond the scope of this work. However, the simulations clearly indicate that a vortex with ccw chirality is more easily nucleated in the Py than in the Co layer. Considering that the experimental results that 
will be shown later are compatible with the existence of only one vortex in the system, Py layer is considered active with its initial configuration represented by a vortex of ccw chirality. On the other hand, the Co layer, which acts as polarizer, remains in the configuration discussed above (Fig. 4.11, insets $(\mathrm{a}, \mathrm{c}))$. Even though the processes taking place in the Co layer could be different, the final magnetization configuration is very likely to be similar to the one found in the simulation, since the OP profile is determined by the geometry, i.e. the constriction in the vicinity of the PC, whereas the curling in the IP component is determined by the Oersted field.

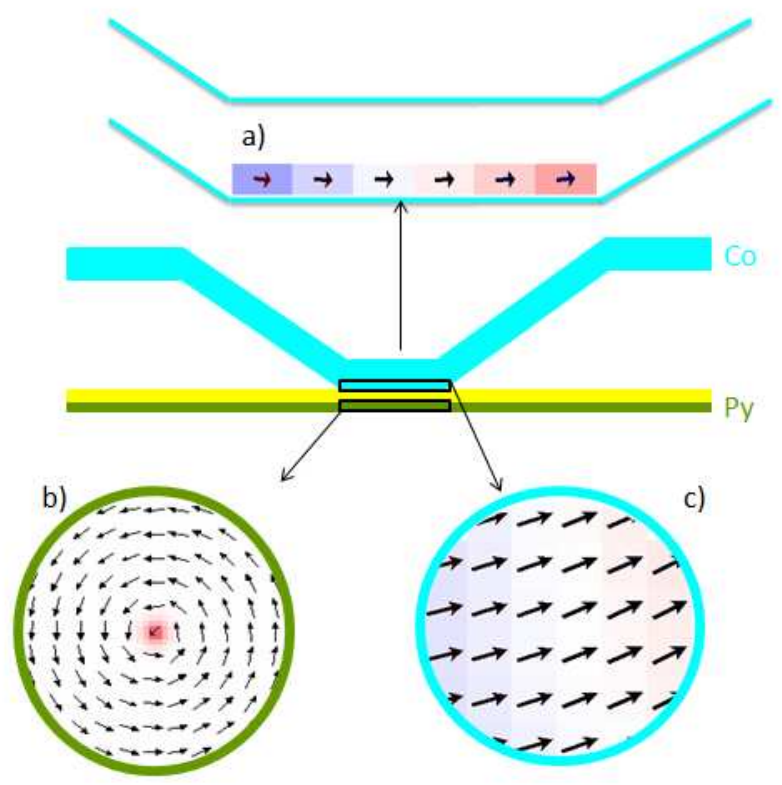

Fig. 4.11: The magnetization configuration: side view of PC in Co layer (a), at the cross-section of Py (b) and Co layer (c) as extracted from simulations accounting for realistic current and Oersted field distribution. 


\subsection{Current dependence}

In order to proceed with the systematic numerical study of the system dynamics, main findings are briefly summarized up to this point. Experimentally the MR oscillations are found only for the positive current direction which corresponds to electrons flowing from the Co to Py layer (i) and the output microwave power excludes the possibility of the coexistence of two vortices, one vortex in each layer, (ii). On the other hand the static simulations indicate the existence of ccw vortex and nonuniform configuration inside the PC in the Py and Co layers, respectively, (iii). Moreover, the large thickness of the Co layer should make it immune to the ST effect from the reflected electrons. For this reasons numerical simulations are to be carried out assuming that the Co layer serves as a static in time polarizer and the Py layer is the dynamically active ferromagnet, i.e. only the dynamics of the latter has to be resolved.

As presented in Fig. 4.12, following results have been obtained under above mentioned assumptions. First, the frequency found numerically (in the $\mathrm{GHz}$ range) is much higher (a) than the experimentally observed one (b) and (c). Second, the higher spectral harmonics, reported in the measurements, are absent in the results from the simulations - due to the nearly circular vortex orbit shape. The first observation shall be complemented with the fact that the simulated orbit of the gyrating vortex in $\mathrm{Py}$ is found to be inside area under the PC, thus resulting is the negligibly small GMR signal. This again contradicts the experiment.

These disagreements lead to the conclusion that some important factors are missing in the model. Reconsider the thick Co layer. Up to now its role was limited to defining polarization for the ST introduction. Thus, $15 \mathrm{~nm}$ thick layer made of ferromagnetic material is a source of significant magnetostatic field that could, at least in principle, influence the dynamics of the thin Py layer. This contribution has been calculated micromagnetically 
and is presented in the cross-section corresponding to Py layer in Fig. 4.13b. The arrows represent the IP projection of the field, whereas the perpendicular component is in color scale. As can be observed, the IP component of the field is predominantly along the direction opposite to the magnetization in Co, whereas the OP component changes from negative (blue) to positive (red) as we move from right to left in the region close to the $\mathrm{PC}$.
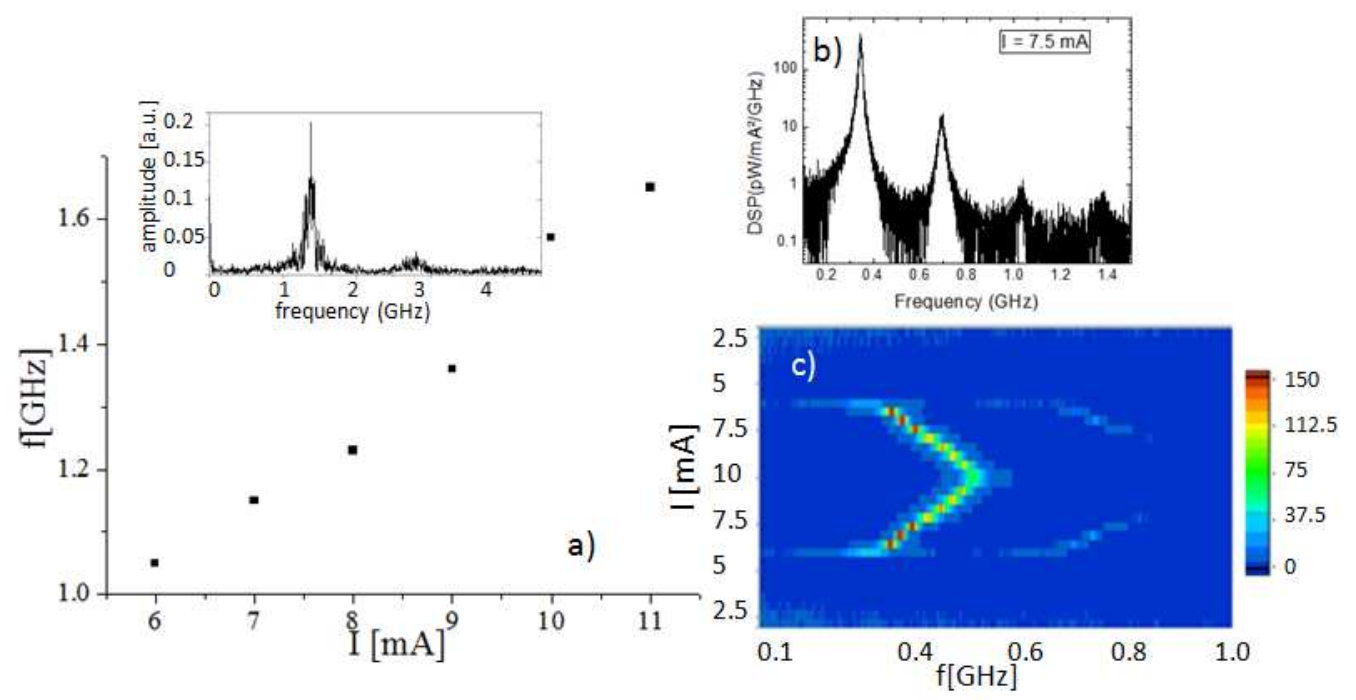

Fig. 4.12: Frequency blueshift with current (a) as observed assuming initial state from Fig. 4.11. Only the dynamics of vortex in Py is resolved and Co layer is assumed to be static in time polarizer. An example frequency spectrum in the inset. Experimental spectrum at $7.5 \mathrm{~mA}$ (b) and in color map in whole reported current range (c).

In Fig. 4.14a the sum of Oersted and stray fields in the Py layer is shown for the applied current of $\mathrm{I}=7.5 \mathrm{~mA}$ by means of an arrow plot and a blue-white-red color scale for its module. As can be observed, the stray field 
breaks the rotational symmetry of the Oersted field. Therefore, the positiondependent potential for the vortex is modified loosing its rotational symmetry with respect to the $\mathrm{z}$-axis. In particular, the minimum energy position for the vortex is no longer the center of the nanocontact but it is displaced outside to the position highlighted as red dot close to the nanocontact in Fig. 4.14b.

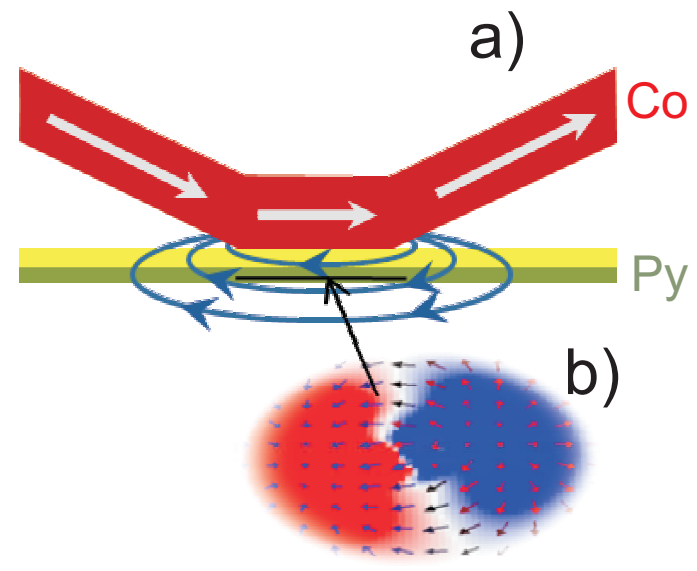

Fig. 4.13: Schematic representation, (a), and the arrow plot of the stray field created by the Co layer on the Py layer, (b). The color scale in (b) corresponds to the perpendicular component of the stray field, where red, blue and white mean $\mathrm{z}>0, \mathrm{z}<0$ and $\mathrm{z}=0$, respectively.

When the dynamic simulation is carried out including the ST, the vortex, initially positioned exactly at the $\mathrm{PC}$, is expelled from it until it reaches a stationary orbit with the frequency of $\mathrm{f}=380 \mathrm{MHz}$. This orbit, plotted in Fig. 4.14b, is neither circular nor symmetric with respect to the nanocontact position. As a result, the spectrum of the voltage signal is very rich in higher harmonics, Fig. 4.14c. The color map of the OP component of the stray field at the level of the Py layer is presented in inset (b) of Fig. 4.13. The profile is highly nonuniform due to the cone-shape constricted geometry of the Co 
layer in the vicinity of PC. The vortex core shift - due to the stray field in this case - is compatible with the experiments [77], where the vortex response to the applied field was studied. This feature can be explained as follows: the presence of a nonuniform stray field changes the energy landscape of the Py layer magnetization and, therefore, the lowest energy position of the vortex does not coincide with the sample center anymore. This means, that not only the ST but also the stray field contributes to the expulsion of the vortex from the area below PC. Finally, when current is applied, it induces vortex motion around its equilibrium position as discussed above.
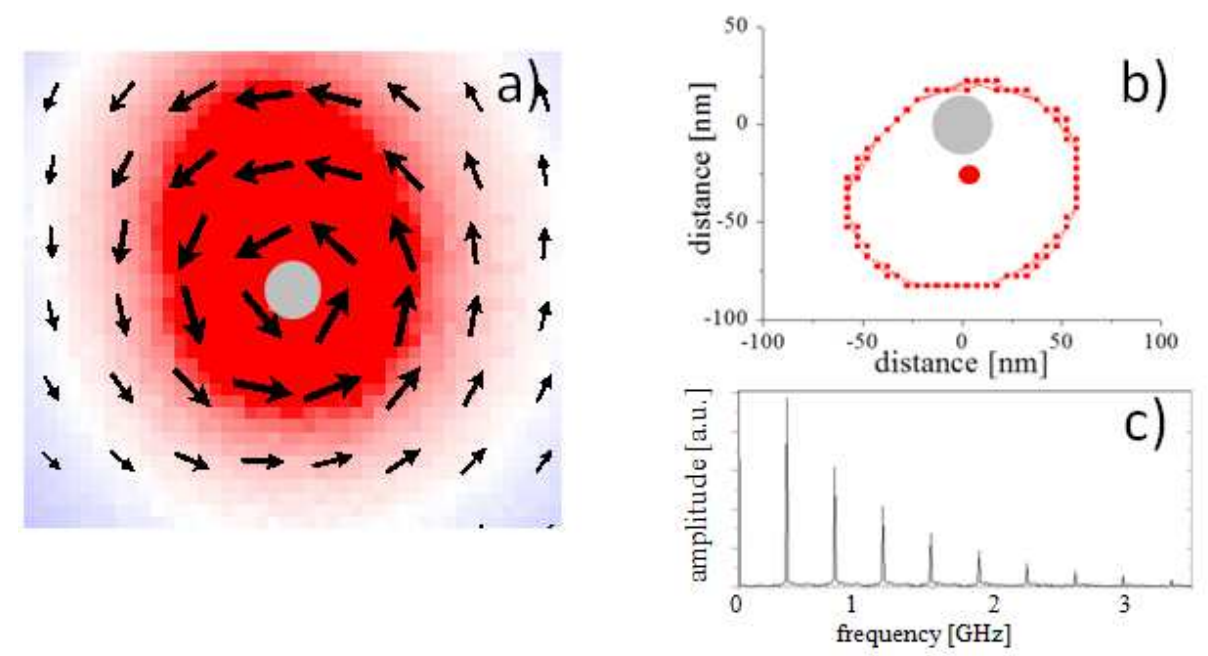

Fig. 4.14: Arrow plot of the sum of the Oersted $(\mathrm{I}=7.5 \mathrm{~mA})$ stray fields in the vicinity of the nanocontact (highlighted in grey) in the Py layer, (a). The strength of the total field is represented with a blue-white-red color scale. Computed trajectory of the vortex core, (b). The nanocontact is highlighted in grey, whereas the red dot indicates the equilibrium position (in absence of ST) for the vortex. Frequency spectrum of the voltage signal, (c). 
Systematic simulations were carried out for different values of the applied current and similar behavior to the one described above was found in all cases. The frequency of the main peak in the spectrum of the voltage signal is plotted as a function of the current and compared to experimental data in Fig. 4.15. The experimental data were measured by sweeping down the current. As can be observed, an excellent quantitative agreement between the simulations (open red circles) and the experiment (open black squares) is found, showing an approximately linear frequency increase with the slope of $\frac{\mathrm{df}}{\mathrm{dI}}=57 \mathrm{MHz} / \mathrm{mA}$. Therefore, one concludes that zero-field vortex oscillations are sustained due to the combined action of the ST and the stray field, both of which are determined by the magnetization configuration in the Co layer. The crucial role played by the stray field in this process shall be emphasized at this point. Recall, that when this term was not taken into account in the simulations, the vortex was found to move inside the PC, leading to a low amplitude output voltage signal at much higher frequency.

Moreover, as indicated by the simulations, the amplitude of the output GMR signal increases with current close to the threshold and then remains constant up to the critical current (full red circles), Fig. 4.15. This is again in qualitative agreement with the experimental findings (not shown).

Furthermore, the displacement of the vortex with respect to the sample center is found to be current dependent. At low currents (lower frequency) the vortex is displaced further away than at larger currents as indicated in Fig. 4.16a. Compare at $5 \mathrm{~mA}$ and $10 \mathrm{~mA}$. Though, below the threshold current associated attractive potential created by the Oersted field is not strong enough to counterbalance the ST which, therefore, expels the vortex far away from the contact. In the simulations, at very low currents due to confinement in the computational size, the displacement of the vortex reaches critical maximum which is associated with the bean shaped orbit as represented at $1 \mathrm{~mA}$ in black in Fig. 4.16a. This is traced back to the profile of the stray field, which changes the energy landscape across the sample. 
Thus, at certain current different lowest energy valley is defined for vortex oscillations to be sustained on a closed orbit. Hence, within one period the vortex gets to be displaced very far from the PC and then it passes through it. This feature can be also seen by considering the output signal. In case of vortex gyrating entirely outside the PC one obtains full signal as presented in green for $5 \mathrm{~mA}$ in Fig. 4.16b. On the other hand, vortex passing below the PC at some stage of the orbit reflects in the decrease of the signal as presented in black for $1 \mathrm{~mA}$. Note, that the numerical threshold current of $1 \mathrm{~mA}$, obtained when decreasing the current, is surprisingly low as compared to the experimental value of $6 \mathrm{~mA}$. This can be explained as follows. Considering that at low currents the vortex is expelled very far away from the PC area (Fig. 4.16a) one can argue that possible sample defects pin and immobilize the vortex hindering output signal.

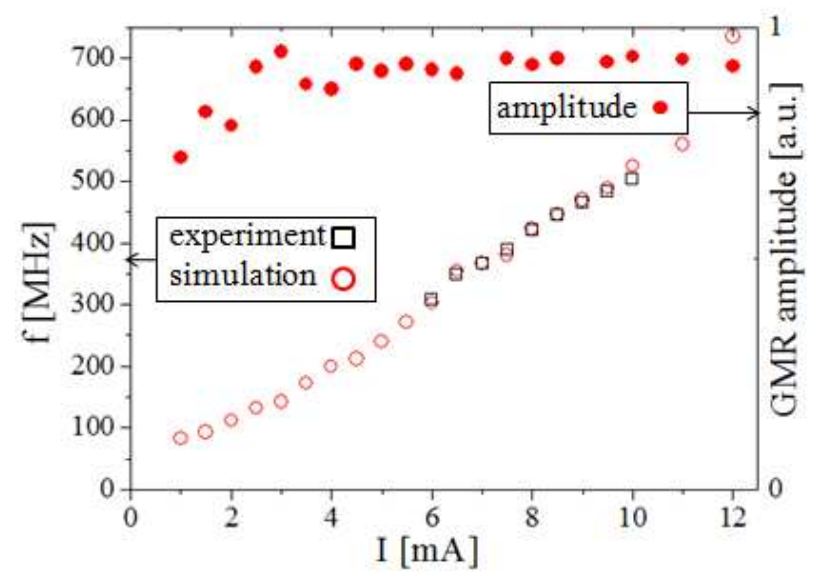

Fig. 4.15: Left axis: frequency of the main peak as a function of the applied current. The results obtained from micromagnetic simulations, red open circles, are compared to experimental data, black open squares, which have been obtained sweeping down the current. Right axis: the amplitude of the output GMR signal as extracted from simulations, red full circles. 

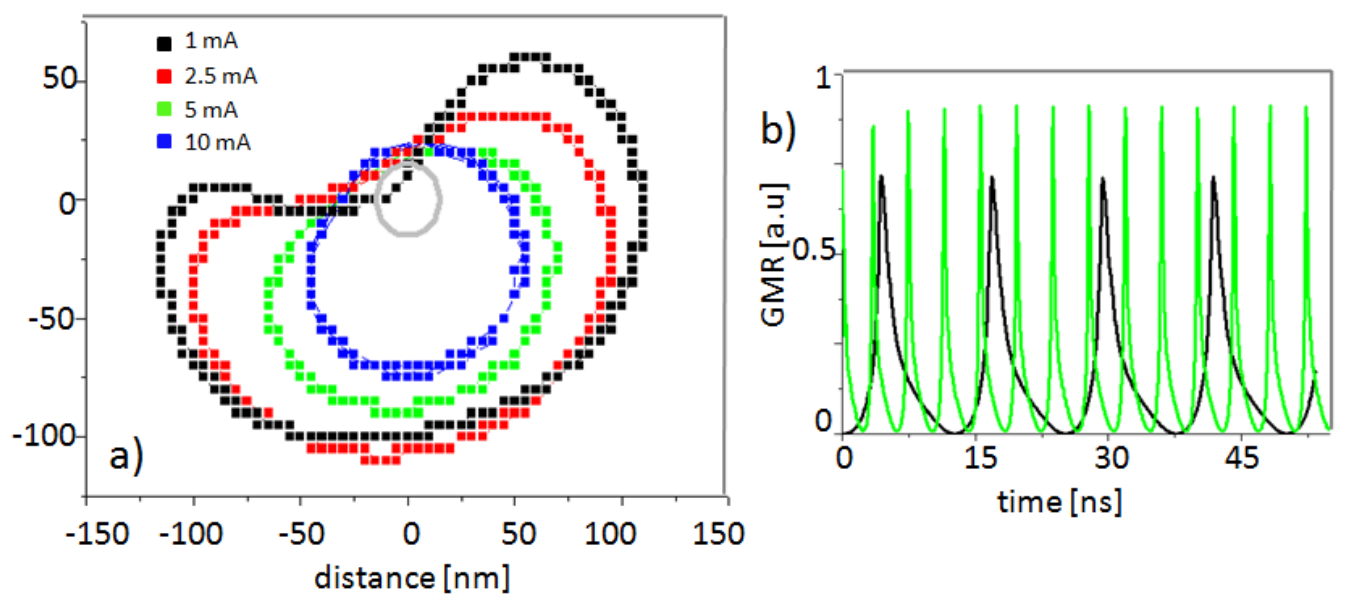

Fig. 4.16: Displacement of the vortex at different currents, (a). At low currents vortex displacement is large and the bean shaped orbit is observed as a result of the energy landscape modified by the inhomogeneous stray field. GMR signal as extracted at $1 \mathrm{~mA}$ and $5 \mathrm{~mA}$, (b), indicating vortex orbiting entirely outside PC in green and partly crossing PC in black. The same color legend for both subfigures.

The critical current, on the other hand, corresponds to the following situation. With the current increase the velocity of the ST driven vortex increases. Thus, at some point the critical velocity for vortex core reversal is reached and the vortex decays to PC. At the same time the GMR output amplitude drops. Indeed in the simulations above the current of $12 \mathrm{~mA}$ the vortex is pushed back toward the PC where it undergoes periodic polarity changes. This is followed by the nucleation of spin waves which requires the employment of proper $\mathrm{ABC}$, upward jump of the frequency (not shown) and the decrease of the output power. At $20 \mathrm{~mA}$ the reported frequency reaches $5.789 \mathrm{GHz}$ and considering that spin wave generation originating from the 
periodic vortex core polarity switching is not the subject of this study the results are presented only up to $12 \mathrm{~mA}$.

Above explanation is compatible with the experimental finding that the output power first increases with current to finally remain constant at larger currents (trend reported when decreasing the current). This effect, as indicated by the simulations has to do with the current dependent vortex displacement. Hence, it provides qualitative justification for the existence of the threshold and the critical currents, not accounted for in the rigid vortex model [39].

\subsection{Field dependence}

In this section the sample is first considered in saturation in the negative z-direction. The configuration change in Co layer, as well as the associated stray field response to the increasing external field is to be determined. These crucial contributions are updated for each value of the applied field are then accounted for in the study of vortex dynamics in Py layer.

Thus, the initial state is obtained as described in the previous paragraphs with the difference that the negative applied saturating field favors now negative polarities of vortices. Vortex nucleated in the Py layer preserves its negative polarity throughout the studied field range. It is expelled from the nanocontact as a combined effect of the ST and the stray field and its movement leads to well defined oscillations. An excellent quantitative agreement is found between the experimental data and numerical results. As shown in Fig. 4.17, the frequency first decays to reach the minimum and then increases with the external field. In order to qualitatively explain this nonmonotonic frequency dependence on the field value we have investigated the evolution of the magnetization in the polarizer and with its associated stray field.

Side views of the magnetization state in the nanocontact in Co layer are presented in insets of Fig. 4.17 for the external fields of $-50 \mathrm{mT},-17 \mathrm{mT}$ 
and $27 \mathrm{mT}$. It can be clearly seen that even at significant negative fields the configuration is highly nonuniform with large positive and negative OP components.

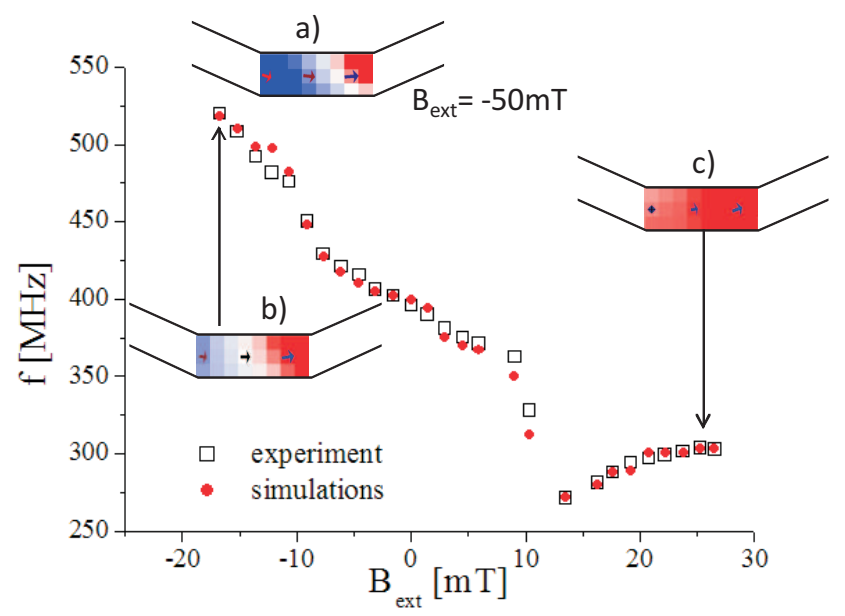

Fig. 4.17: Dependence of the vortex mode frequency on the strength of the applied OP field, after saturation at large negative field. The magnetization configuration in the nanocontact in the polarizing Co layer evolves with the applied field. The insets present in color scale the OP magnetization component at fields of $-50 \mathrm{mT},-17 \mathrm{mT}$ and $27 \mathrm{mT}$. The negative OP component tends to be suppressed as the field is increased.

The frequency first decays as the applied field is increased. Thus, the above discussed OP components, arising from the the cone-shaped constriction in Co layer, tend to be suppressed as the field is increased. Numerically the field term in the LLG equation accounts for the nonuniform stray field but it is useful to make an average of its cartesian components and monitor its overall average magnitude. As presented in Fig. 4.19 in the range discussed (marked I) the average $\mathrm{y}$-component (red full circles) increases while the absolute value of the average $\mathrm{x}$-component (open black squares) 
decreases. The latter is approximately three times larger and, therefore, the decrease of the overall magnitude of the average stray field (blue diamonds) is observed as a consequence of the decrease of its dominating $\mathrm{x}$-component. This decrease in the field term in turn is reflected in the frequency decrease with the external field.

After crossing $0 \mathrm{mT}$ both the $\mathrm{OP}$ magnetization component of the polarizer as well as the average OP stray field component become antiparallel to the vortex core polarity. As presented in Fig. 4.19 (range II) the magnitude of the average stray field is observed constant owing the change of the sign of its $\mathrm{y}$-component whose contribution to the overall average SF is increasing. In the following full model will be referred to as the simulations where both, the stray field as well as ST, are accounted for in their nonuniform form. Thus, simulations have shown, that if in this case the inhomogeneous profile of the stray field is neglected, then no steady-state oscillations are supported at all. In this particular test simulation the average stray field was estimated (as from Fig. 4.19) and implemented as an uniform contribution to the field term in the LLG equation. No oscillations are observed in that case, which indicates that the damping is no longer counterbalanced by the ST. This, in turn leads, to the conclusion that the nonuniform character of the stray field plays crucial role in sustaining the microwave output at low external field. Hence, if the same averaging procedure is done with respect to the ST term qualitatively the same behavior is observed as in the case accounting for all the inhomogeneities (full model). Moreover, even if a perfectly IP polarization of the electrons is assumed (along $+\mathrm{x}$-direction, i.e. neglecting any possible OP component) but the stray field is kept in its original form qualitatively and quantitatively the same frequency is obtained as arising from the full model. Hence, the ST from the nonuniform polarizer configuration is proven to be a secondary effect compared to the nonuniform stray field.

Consider, that the inhomogeneous stray field comes from the locally constrained cone-shaped Co layer, which results in the appearance of magne- 
tostatic charges on the side surface of the cone. These charges are simply not present in a standard flat geometry and, therefore, the dynamics observed in the hybrid sample is largely determined by the nontrivial sample geometry. Hence, the experimentally reported frequency decrease with field corresponds to the off-centered vortex oscillations driven by a combined effect of the damping accounting for the external field and a nonuniform stray field term counterbalanced by a nonuniform ST.

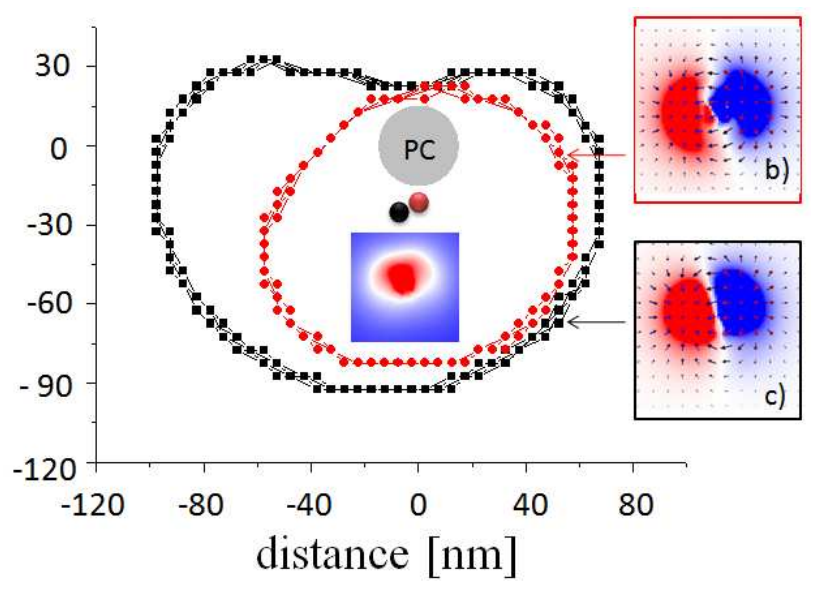

Fig. 4.18: Vortex orbit at $0 \mathrm{mT}$ (red circles) in at $19.19 \mathrm{mT}$ (black squares) together with the z-component of the stray field, displayed in the insets (b) $0 \mathrm{mT}$ and (c) $19.19 \mathrm{mT}$. Stray field magnitude at $19.19 \mathrm{mT}$ in the middle inset. Nanocontact area is marked in grey. The red and black dots near the PC area indicate the vortex positions if no ST is present at $0 \mathrm{mT}$ and $19.19 \mathrm{mT}$.

Above $10 \mathrm{mT}$ both the polarizer magnetization configuration and the magnitude of the stray field close to nanocontact (middle inset of Fig. 4.18) become more uniform. In particular, the negative OP magnetization component in the polarizer becomes suppressed. Moreover, the maximum displacement of the equilibrium position of the vortex (black dot in Fig. 4.18) 
is observed, resulting in the lowest oscillation frequency. The mode is also affected qualitatively. An elongated orbit whose symmetry coincides with the symmetry of the stray field magnitude, Fig. 4.18, is observed since the weakening of the negative magnetization OP component in the polarizer allows the negative polarity vortex to move to the left of the PC.

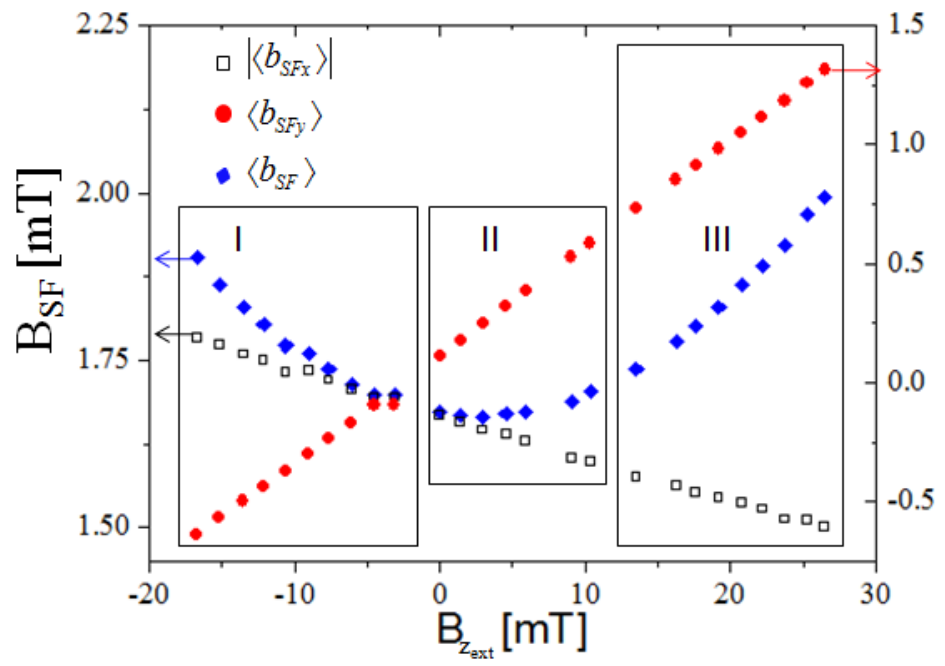

Fig. 4.19: Evolution of the IP components of the average stray field with the applied OP field. The y-component (red full circles) increases contrary to the absolute value of the $\mathrm{x}$-component (open black squares). In the range marked I the decrease of the overall magnitude of the stray field (blue diamonds) is driven by the decrease of the dominating $\mathrm{x}$ component. In range II constant average of the stray field is observed owing the change of the sign of the $\mathrm{y}$-component and its increasing contribution to the overall average SF. In range III the increase of the overall average SF with the external field is observed as a consequence of the increase of its both IP components.

Above this critical field $(10 \mathrm{mT})$ quantitatively similar behavior is reported even if the inhomogeneous character of the stray field is not accounted 
for, since the IP component of the stray field starts to dominate its overall magnitude. This IP stray field component increases as the field is increased resulting the increase of the overall average stray field magnitude and the frequency nonlinear blueshift with field in this range.

Reversing the polarity of the vortex, changes the magnetostatic field within Py layer. As presented in Fig. 4.20, the equilibrium position in absence of ST is shifted as the polarity is flipped. Compare red central dot for positive and blue central dot for negative polarity vortex. The corresponding orbits are both off-centered but shifted in opposite directions resulting in different frequencies (437 MHz and $394 \mathrm{MHz}$, respectively). It has been recently demonstrated that the existence of two stable gyrotropic modes of vortex core having different polarity result in different rotation frequencies [78]. Comparing Fig. 4.20 to the color map the OP component of the stray field, Fig. 4.18b, one concludes that the orbit of vortex with negative polarity is shifted to the right from the PC, where the stray field $\mathrm{OP}$ component is also negative. On the other hand, in case of vortex with positive polarity the associated orbit is shifted leftwards, where positive OP component of the stray field is present. Clearly the origin of this polarity dependent shift can only be magnetostatic. Note, that the STT affects only the magnetization inside the PC irrespective of the core polarity of the vortex gyrating outside the PC. Hence, from the magnetostatic point the better the alignment of the external field with the core polarity the lower vortex displacement and in what follows with it associated energy cost [77]. That is the reason why the trajectory of a vortex with negative core polarity is more confined on the side where the positive, and more elongated where the negative, OP stray field is present. 


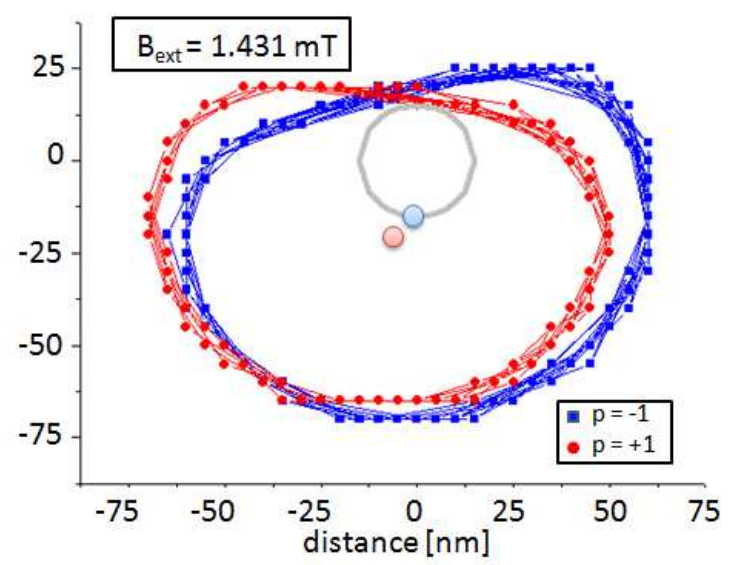

Fig. 4.20: Orbit at $1.431 \mathrm{mT}$ positive OP field compared for a vortex of a positive (red) and negative (blue) polarity. The corresponding frequencies are: $437 \mathrm{MHz}$ and $394 \mathrm{MHz}$, and the equilibrium position (without ST) are marked with red and blue dot respectively.

\subsection{Conclusion}

Within this chapter a broad numerical study of the ST induced magnetization dynamics in the trilayer system $\mathrm{Py} / \mathrm{Cu} / \mathrm{Co}$ in the $\mathrm{PC}$ geometry with the nontrivial locally constricted profile of the polarizer (thick Co layer) has been considered. The results [65] lead to the conclusion that the ST-induced dynamics in such a contact is governed by the formation and rotation of the magnetization vortex in a flat (thin) layer. Calculations of the realistic current distribution in this contact and incorporation of the corresponding Oersted field allowed for the determination of the lowest energy configuration in the sample, defining the Py to be the active layer. This result clearly indicates that in ST-based oscillators the vortex nucleation process depends on the exact distribution of the current and associated Oersted field, rather than on the layer thickness.

Further systematic numerical study of the frequency dependence on the current strength provided not only the qualitative explanation of the experi- 
mental observations, but also resulted in an excellent quantitative agreement between simulated and measured data. In particular, the Co layer stray field acting on the Py layer has been demonstrated to strongly influence the magnetization dynamics, deforming the vortex orbit which resulted in the appearance of significant higher harmonics in the oscillation power spectra. Hence, vortex expulsion from the area below the PC was shown to be the combined effect of the ST and the stray field. Additionally, the dependence of the vortex displacement on the current has been predicted, thus explaining the existence of the threshold and the critical currents for the steady-state oscillation regime.

Finally, numerically obtained system response to external field is also in an excellent agreement with the measured data. It has been shown that at low external fields, the highly inhomogeneous stray field plays the crucial role in the excitation of vortex mode. Taking into account that this stray field comes from the locally constrained geometry of the Co layer, the geometry-driven vortex mode is believed to have been observed. This result demonstrates a novel way of tailoring vortex STNOs by manipulating the geometry of the pinned layer. 


\section{INTERLAYER COUPLED VORTICES IN SINGLE POINT CONTACT}

This chapter is dedicated to the study of a flat spin valve structure in point contact geometry with no exchange bias. The sample investigated in the previous section is reconsidered, thus, now with the flat profile of both layers. Hence, in this case the Oersted field shows to favor nucleation of two vortices, one in each ferromagnetic layer, of the same chirality. For the systematic study the thickness of Co layer is reduced and a trilayer consisting of $\mathrm{Co}(5) / \mathrm{Cu}(5) / \mathrm{Py}(5)$ is investigated. Limiting cases of ST active on each one of the layers separately and simultaneously are studied indicating the possibility to manipulate the frequency by proper design of the structure (favoring or blocking ST effect on one of the layers). Additionally, a specific trilayer is considered, $\mathrm{F}_{1}(5) / \mathrm{Cu}(5) / \mathrm{F}_{2}(5)$, where both ferromagnetic layers are made of the same material.

\subsection{Introduction to double vortex oscillator.}

As far as vortex based STNO, it has been shown in a vortex based STNO that the OP component of the magnetization in the pinned layer is essential for the vortex to be excited into steady motion [39, 43]. Very recently it has been predicted that also a nonuniform IP magnetized polarizer [41] can excite current driven vortex dynamics. Still, in many of the studies so far the polarizer has been assumed to be static in time. To fulfill this assumption in the historically first STNO the spin valve was fabricated with the pinned layer 
made ferromagnetic material characterized by large saturation magnetization and/or much thicker than the free layer. Significant thickness assures that the STT, active only at the interface [72], influences only the magnetization in the tight vicinity of the interface and, therefore, prevents dynamical evolution of the magnetization across the whole thick layer. Moreover, the use of ferromagnets of large saturation magnetization in the design of pinned layers further decreases the effect of ST exerted on it as it is inversly proportional to the saturation magnetization of the material.

Additionally, layer designed for the reference (pinned layer) might be fixed by introducing exchange bias or synthetic antiferromagnet (SAF) pining. The latter is the state-of-the-art solution for pillar structures and provides up to $200 \mathrm{mT}$ of biasing field. However, SAF design encounters number of technical difficulties to be successfully applied in the PC geometry. Therefore, most of the PC devices investigated experimentally up to now were fabricated with the IrMn layer giving up to $50 \mathrm{mT}$ of pinning bias.

Since the model [6] requires the polarizer to be static the concept of magnetization dynamical evolution in both ferromagnetic layers has not been explored for a long time. The idea of two active layers became interesting first when vortex based STNO started attracting lots of attention. Only very recently the possibility of both magnetic layers supporting vortex configuration in a pillar geometry has been investigated [46]. Moreover, it has been demonstrated that synchronization of two oscillators in such systems leads to great improvement in terms of reported linewidth [15]. No study of such STNO pair in PC geometry has been performed so far. Therefore, this chapter is dedicated to modeling of a trilayer PC structure where both ferromagnetic layers are found to be in a vortex state.

\subsubsection{Geometry and convention}

The system under study is a trilayer $\mathrm{F}_{1} / \mathrm{Cu} / \mathrm{F}_{2}$ in a $\mathrm{PC}$ geometry with the contact radius of $20 \mathrm{~nm}$. None of the layers is exchange biased, so that 
both layers can, at least in principle, evolve dynamically. In the convention adapted hereafter electron flow from bottom to top defines positive current direction and both current directions are investigated. First, $\mathrm{F}_{1}=\mathrm{Co}$ and $\mathrm{F}_{2}=\mathrm{Py}$ are chosen for the study presented below.

In general, vortex nucleation can be favored in a ferromagnetic layer whose thickness exceeds the exchange length and provided that the aspect ratio (thickness/lateral size) is kept small [74]. The latter is always fulfilled in a PC design as it is a laterally extended geometry. However, simulations of such structures are limited to certain computational sizes and are, in general, very time consuming. Therefore, lateral size of $1 \mu \mathrm{m} \times 1 \mu \mathrm{m}$ is chosen for further study with the $5 \times 5 \times 5 \mathrm{~nm}^{3}$ mesh and single mesh layer of Py as shown in Fig. 5.1 is considered. To check the prediction of thickness constrained discarding of the ST induced dynamics in thick layers, first $\mathrm{t}=15 \mathrm{~nm}$ Co layer is assumed and some general qualitative study is carried out. Since STT is a purely superficial effect [72] in $15 \mathrm{~nm}$ of Co it is active only in the vicinity of the $\mathrm{Cu} / \mathrm{Co}$ interface. From the computational point of view it imposes severe constraints on the mesh in $\mathrm{z}$-direction. Dealing with these issues, however, is far beyond the subject of this chapter. Therefore, an alternative approximation is proposed i.e. numerically the torque is only implemented to the top of the Co layer which is represented as a shade in Fig. 5.1. This approach should, at least partly, offset the uncertainty in the frequency estimation.

Note that in terms of quantitative study full micromagnetic modeling of this structure leads to prohibitively long computational times. Hence, for further systematic study the thickness of Co layer is decreased to $t=5 \mathrm{~nm}$ yielding results in reasonable computational time frame.

Theoretical description of STT in structures with two different ferromagnetic materials has not been delivered yet. Neither the case of two active ferromagnetic layers has been treated theoretically in the ballistic transport limit. Therefore, in the first assumption, Slonczewski - like torque term [6] 
from the existing model, accounting for a fixed polarizer, is considered in equal from for both layers. Detailed description of the numerical model is to be found in Ref. [46]. In the following the limiting assumptions of torque acting separately either on Py or Co and finally on both layers simultaneously are investigated.

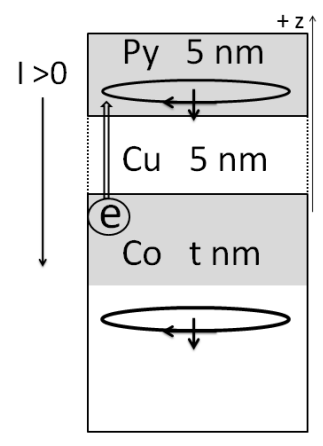

Fig. 5.1: The geometry of studied system. In the convention adapted the electron flow from Co to Py defines positive current direction. Shaded areas define torque sensitive part of the layer. As the initial state for dynamical study two vortices of the same chirality and polarity are assumed.

\subsubsection{Simulation parameters and methods}

Saturation magnetization, exchange constant and damping constant were set to $0.64 \cdot 10^{6} \mathrm{~A} / \mathrm{m}, 1.3 \cdot 10^{-11} \mathrm{~J} / \mathrm{m}$ and 0.01 for Py and $1.4 \cdot 10^{6} \mathrm{~A} / \mathrm{m}, 3.0 \cdot 10^{-11} \mathrm{~J} / \mathrm{m}$ and 0.01 for Co, respectively. Sixth order Runge-Kutta solver was employed for time integration. ST (if applies) is exerted only on one mesh layer.

For this structure no current and associated Oersted field distribution calculations are performed. The reason is twofold. First of all both layers are assumed flat indicating that no complex current distribution is to be expected. Second, no experimental report on such structures is available. Without experimental specification of the exact fabrication process 
and material parameters uniform current distribution and infinite wire approximation for the calculation of the Oersted field seem to be good first approach to the problem that has never been studied. Thus, considering arguments presented in [79], that the infinite conductive wire approximation always highly overestimates the realistic distribution, a correction factor of 0.4 is introduced to the strength of the Oersted field.

\subsubsection{Nucleation and torques}

We start with $\mathrm{Co}(15 \mathrm{~nm}) / \mathrm{Cu}(5 \mathrm{~nm}) / \mathrm{Py}(5 \mathrm{~nm})$. The sample is initially saturated in negative $\mathrm{z}$-direction (sign convention as in Fig. 5.1) and then the system is allowed to evolve to lowest energy state in absence of external field, at the current of $10 \mathrm{~mA}$ and with ST active in both layers. As a result two vortices of the same chirality and the same polarity are nucleated as schematically shown in Fig. 5.1. Nucleation process at negative current evolves similarly yielding, however, opposite chirality of vortices.

The STT, depending on the current direction, in a spin valve where both ferromagnetic layers are in vortex state, acts either stabilizing or destabilizing on each of the configurations [16]. Let us first concentrate only on the IP polarization acquired by the electrons corresponding to positive current. In this case electrons proceed from cw curled vortex in Co layer where they polarize and impinge on Py, Fig. 5.2a. In general, there is some remaining net torque since the cores are not identical but in practice cw vortex in Py is stabilized. On the other hand, electrons reflected from $\mathrm{Cu} / \mathrm{Py}$ interface polarize ccw and act destabilizing back on Co cw vortex, Fig. 5.2b. In this case dynamics can be expected. In order to extract ST contribution to the dynamics in each layer both limiting cases are investigated separately, i.e. ST active only on Py layer and only on Co layer. Finally, the combined ST effect on both layers is studied in order to determine the dominating torque.

When inversing the current direction, analogous to reasoning as in Fig. 5.2, the torque acts stabilizing on Co layer, destabilizing on Py and their compe- 
tition is observed when both enabled.

\subsection{Positive current}

Assume the ST is active only on the Py layer (Fig. 5.2a). As explained above in this case, for the positive current direction, the initial cw vortex in Py layer is subject to the stabilizing ST effect and no dynamics is expected what is confirmed by the results of numerical study.

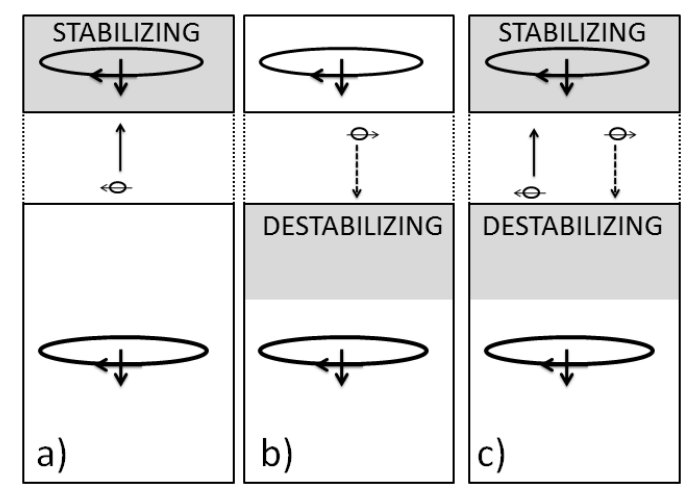

Fig. 5.2: Positive current. Shaded areas indicate ST sensitive regions in case of ST acting on Py (a), Co (b) and simultaneously on Py and Co (c). The transmitted / reflected electrons result in stabilizing / destabilizing effect, respectively.

Now, if only Co layer is subject to ST (Fig. 5.2b) its initial configuration is being destabilized and dynamics, at least in principle, can be expected. Again simulations confirm this prediction yielding picture of complicated dynamical evolution at the test current of $5 \mathrm{~mA}$. Surprisingly, not only the vortex in Co but also the one in Py are expelled from the PC even though no ST effect is active in the latter. 
As shown in Fig. 5.3a less mobile Co vortex moves at the edge of the PC whereas the Py vortex is expelled far from it. The direction of the gyration is $\mathrm{cw}$ in both cases owing the fact that vortex polarity and the sense of its rotation are bonded by right hand rule. Since there is no ST exerted on Py layer the motion of the vortex within must be sustained by the energy flow supported by the temporal evolution of the magnetic configuration in Co layer. The only non-local interaction accounted for in Py layer is the magnetostatic field. Hence, the energy flow essential to maintain gyration of vortex in Py layer is provided by the magnetostatic interactions with ST driven vortex in Co layer. This is a completely novel effect in vortexbased STNO and as such to author's best knowledge has never been studied. Therefore, its implications and consequences are going to be investigated in detail in further sections of this chapter.

Follow the temporal evolution of the average magnetization $\mathrm{x}$-component $\left(\mathrm{m}_{\mathrm{x}}\right)$ inside the $\mathrm{PC}$, as presented in Fig. 5.4a. In the initial time window, Fig. 5.4b, the motion of Py vortex corresponds to a low frequency gyration yielding simple sinusoidal evolution of $\mathrm{m}_{\mathrm{x}}$ (red). On the other hand, in Co layer (black), the $m_{x}$ seem to be a superposition of a low and a high frequency gyration. Thus, the evolving configuration in Py layer affects the dynamics reported in Co layer, while vortex behavior seems to superpose its own high range eigenfrequency over the low frequency present in adjacent layer.

In the final time window, Fig. 5.4c, it becomes clear that the oscillators do not gyrate independently and two clear eigenfrequencies can be distinguished. Still, in absence of STT on Py layer, the vortex within remains displaced further from the PC center than the ST driven vortex in Co layer. Thus, this higher mobility effect is attributed to the different thickness and saturation magnetization of each layer, i.e. thinner and lower $\mathrm{M}_{\mathrm{s}}$ Py layer allows for larger vortex displacement. 

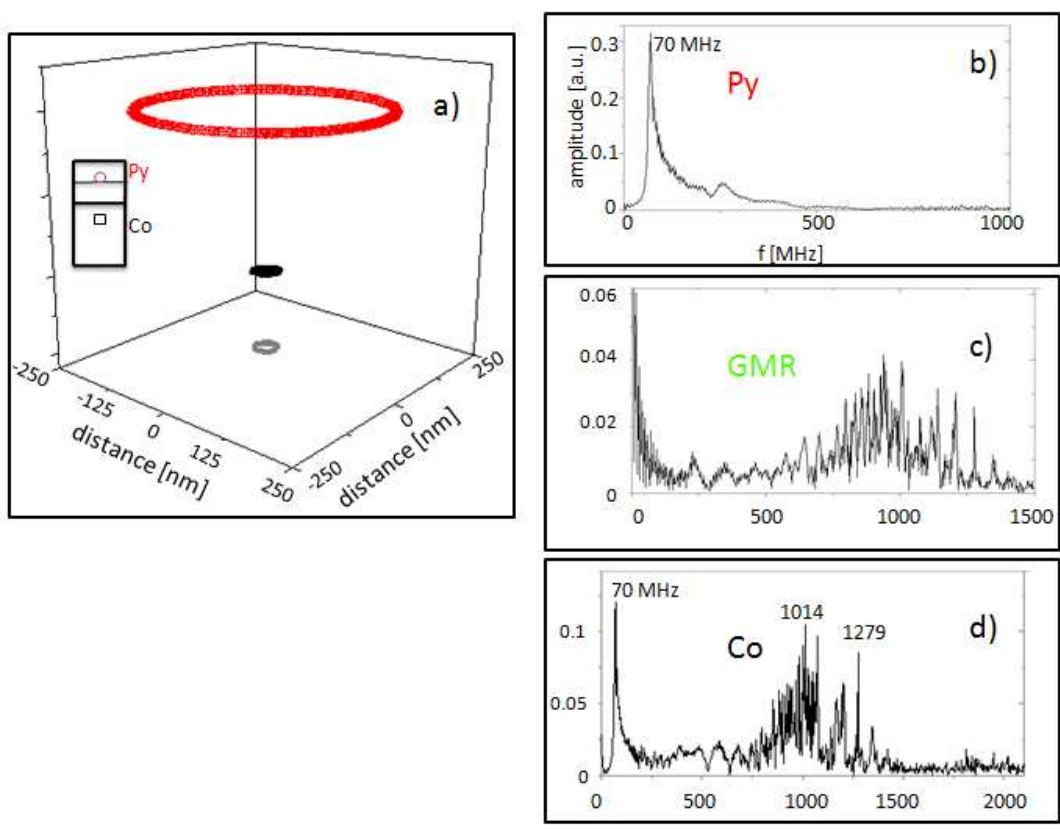

Fig. 5.3: Positive current of $5 \mathrm{~mA}$, ST acting only on Co layer. Orbits of the vortices in Py and Co layers, (a). Frequency spectra as extracted from temporal evolution of the average $\mathrm{m}_{\mathrm{x}}$ component inside the $\mathrm{PC}$, (b) and (d), in Py and Co layer, respectively. Frequency spectrum as extracted from calculated GMR signal, (c).

While the low frequency is found in both spectra, Fig $5.3 \mathrm{~b}$ and $\mathrm{d}$, the high frequency is only present in data set corresponding to Co layer. The resulting GMR spectrum, however, can not be represented easily as the combination of the two above mentioned, Fig. 5.3c.

Interestingly, if one considers ST effect simultaneously on both layers as shown in Fig. 5.2c no dynamics is reported in the studied current range. On one side, the stabilizing effect on the magnetic configuration in Py layer 'pins' its vortex in the sample central position. On the other hand, even though the 


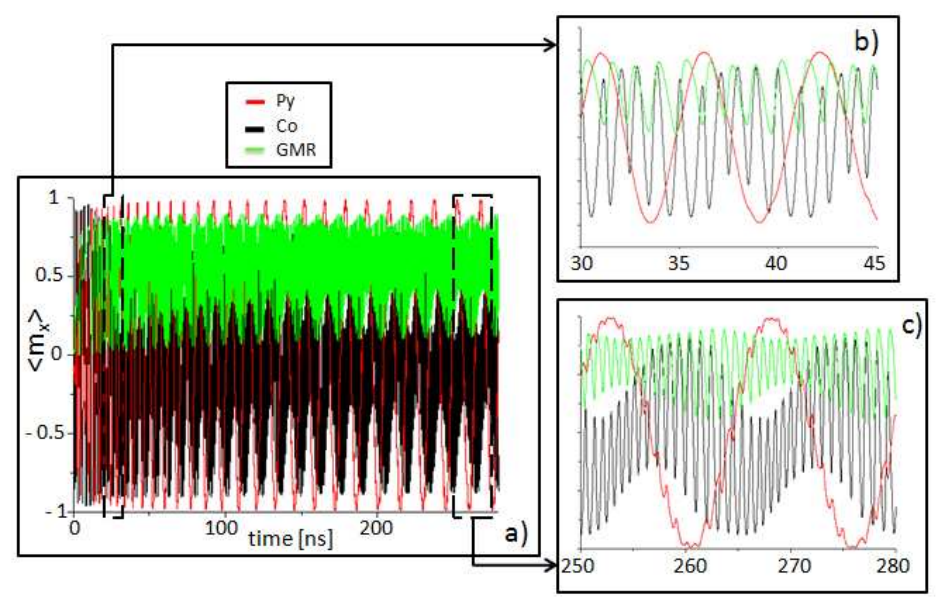

Fig. 5.4: The temporal evolution of the average $\mathrm{m}_{\mathrm{x}}$ component inside the $\mathrm{PC}$ in Py (red), Co (black) and associated GMR signal (green) at the current of $5 \mathrm{~mA}$, (a). Zooms at the initial phase of simulation, (b), and in the final time window, (c).

destabilizing ST effect on Co layer is present, no dynamics is observed. This observation can be explained as follows. Provided that vortex core size in each layer is similar the reflected electrons are polarized almost antiparallel to the configuration in Co, i.e. the angle between the considered magnetization and the orientation represented by the polarizer is close to $180^{\circ}$ where ST amplitude is close to zero. Therefore, its impact is negligible. If any trigger could destabilize this initial angle than the dynamics should be observed.

Note that the dynamics is neither observed assuming ST acting only on Py layer as corresponding to Fig. 5.2a nor when both layers simultaneously are subject to ST as in Fig. 5.2c. This qualitative similarity leads to the conclusion that ST effect on a ferromagnet of lower saturation magnetization is predominant and is activated at lower threshold current. This is going to be confirmed in the following sections. 


\subsection{Negative current}

For this current direction qualitatively similar results are observed when ST active is active on: only Py layer (ST-Py) and on both layers simultaneously (ST-BL) and, therefore, these cases shall be discussed together. Some output is also observed when assuming ST active solely on Co layer.

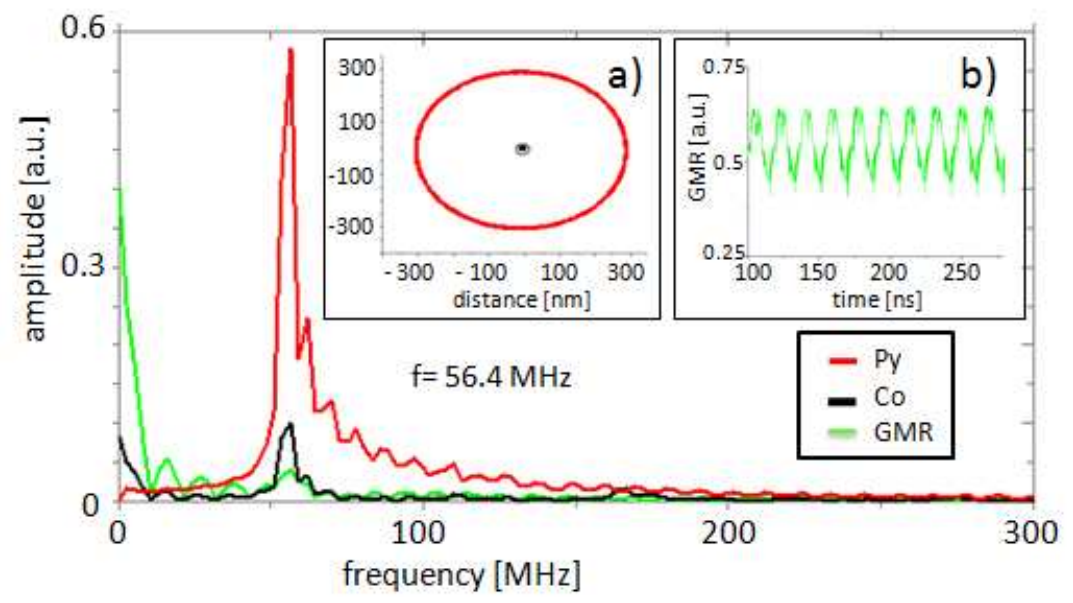

Fig. 5.5: Negative current, ST active only on Py layer. Main figure: frequency spectra as extracted from the temporal evolution of magnetization $\mathrm{x}^{-}$ component inside the PC in Py (red), Co (black) layer and from GMR signal (green) at the current of $-5 \mathrm{~mA}$. Vortex orbit in Py and Co layer in inset (a), PC area marked grey. Temporal evolution of the normalized GMR signal, inset (b). The same color legend for all subfigures.

The orbit of vortex in Py layer indicates that it is expelled far from the $\mathrm{PC}$ as presented in red in insets (a) of Fig. 5.5 and Fig. 5.6 corresponding to ST-Py and ST-BL. In both cases vortex cw gyration outside PC leads to well defined low frequency. On the contrary, much lower amplitude oscillations are observed in Co layer (black) indicating minor cw movements 
of the vortex inside the PC (grey). Therefore, even though vortex in Py layer oscillates well outside the PC area, the movement of the vortex in the adjacent Co layer inside the PC lowers the output GMR signal. Consider main graphs of Fig. 5.5 and Fig. 5.6. The amplitudes of Py (red), Co (black) and GMR (green) frequency spectra show that both vortices move with the same frequency but the resulting output signal is rather low because of the presence of the vortex inside the PC in Co layer. ST acting solely on Py layer leads to the frequency of $56.4 \mathrm{MHz}$, whereas if additionally $\mathrm{ST}$ also on Co is considered the frequency reads $76.7 \mathrm{MHz}$. This difference is the consequence of the change in the energy balance in Co layer caused by the activation of the ST.

Furthermore, the appearance of one sole frequency in the GMR spectra indicates that the oscillators remain synchronized and in ST-BL case the $\mathrm{ST}$ is not effective enough to support second eigenfrequency. This can be understood, since Co layer characterized by high saturation magnetization might not respond to ST effect at current as low as the test $-5 \mathrm{~mA}$. So the frequency corresponds to the vortex moving in Py layer where the displacement throughout the trajectory around the PC reaches $310 \mathrm{~nm}$ ST-Py and $175 \mathrm{~nm}$ ST-BL, respectively. Thus, the displacement is defined by the competition between the ST and the dissipation. The first expels the vortex. Additionally the magnetostatic interactions favor alignment of both cores in $\mathrm{z}$-axis but the strength of this interaction decays with distance. Hence, most probably large core displacement is ST related. One should not discard the possibility that the boundaries of the computational area can be influencing the frequency but this issue is beyond the interest this chapter.

Finally, the impact of ST influencing only the top of Co layer is investigated. For this current direction one expects stabilizing effect of electrons polarized in Py and transmitted into Co. However, considering that the initial vortices in both layers are not identical, in particular there is a difference 


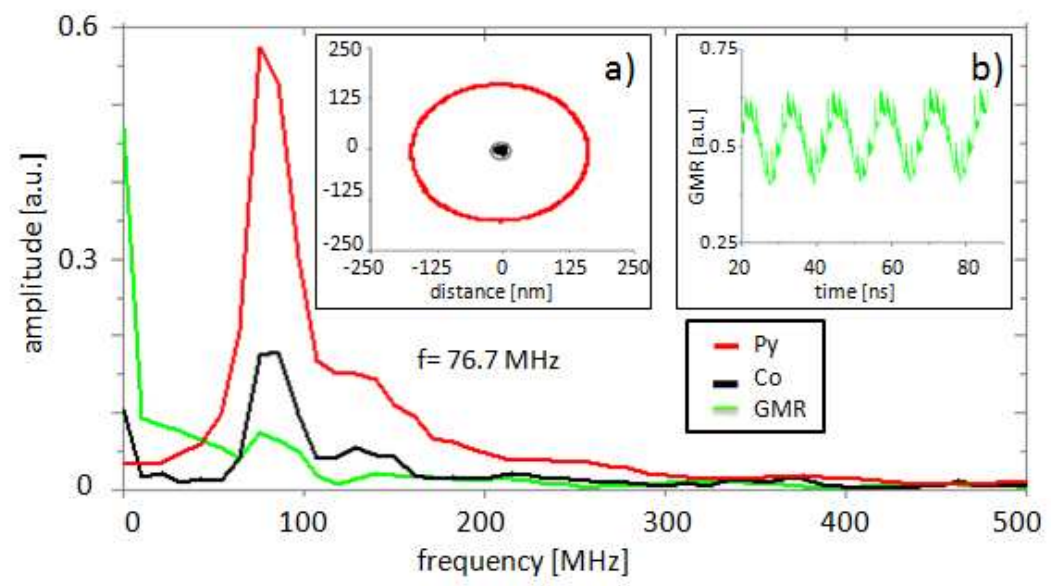

Fig. 5.6: Negative current, ST active both layers. Main figure: frequency spectra as extracted from the temporal evolution of magnetization $\mathrm{x}$-component inside the PC in Py (red), Co (black) layer and from GMR signal (green) at the current of $-5 \mathrm{~mA}$. Vortex orbit in Py and Co layer in inset (a), PC area marked grey. Temporal evolution of the normalized GMR signal inset (b). The same color legend for all subfigures.

in the core volume in each material, there is some net torque remaining. Its presence it then reflected in system dynamic response. As presented in Fig. 5.7a vortex in Co is driven into very low amplitude cw oscillations. This breaking of symmetry magnetostatically triggers vortex in Py to move away from the PC and, owing negative core polarity, oscillate in cw direction. Since no ST is accounted for in the Py layer it is the change in the overall magnetostatic field caused by the magnetization dynamics in Co layer that couples the vortex in Py to oscillate with the same frequency $(570 \mathrm{MHz})$. Thus, low amplitude vortex oscillations in Co layer, i.e. its presence inside the PC, induce rather low output GMR signal as indicated in Fig. 5.7b. 


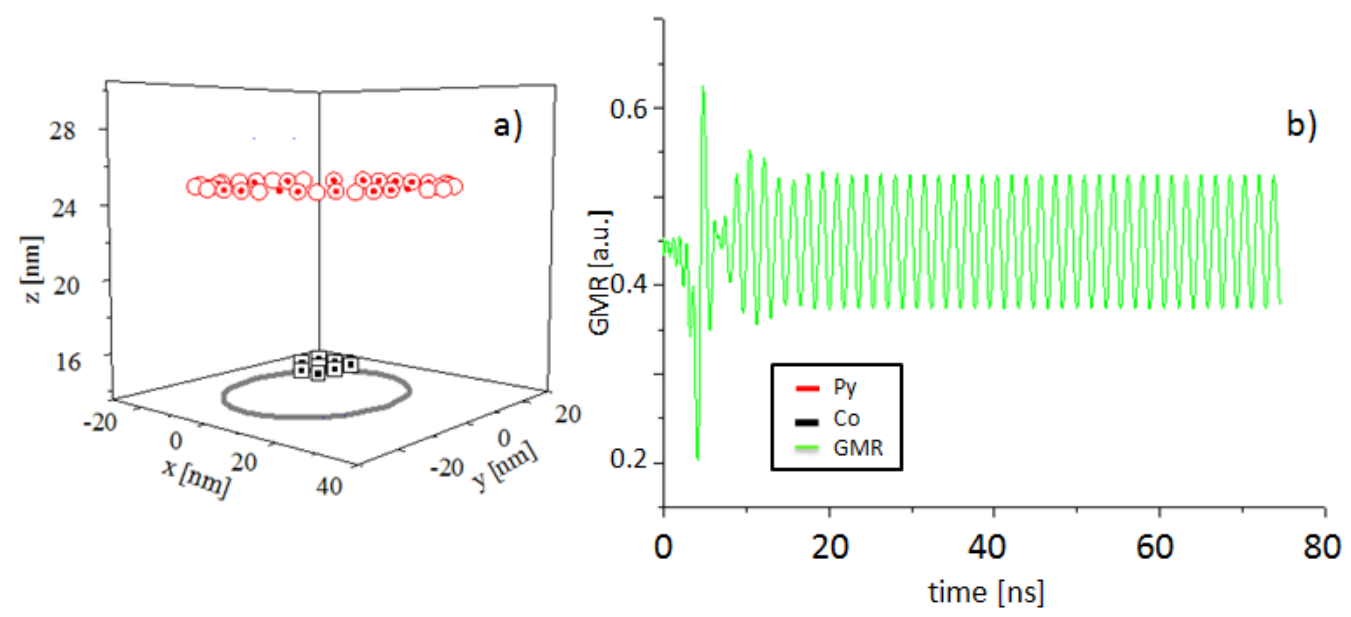

Fig. 5.7: Negative current of $-5 \mathrm{~mA}$, ST active on Co layer. Vortex orbit in Py layer (red) and Co layer (black), with PC marked grey, (a). The normalized GMR signal, (b).

\subsection{Systematic study, $\mathrm{Co}(5 \mathrm{~nm}) / \mathrm{Cu}(5 \mathrm{~nm}) / \mathrm{Py}(5 \mathrm{~nm})$}

Previous section dealt with the system where Co layer thickness was relatively large and, therefore, only a qualitative study could be carried out in the reasonable time frame. To perform systematic numerical study, in the following, the Co layer thickness is decreased and a $\mathrm{Co}(5 \mathrm{~nm}) / \mathrm{Cu}(5 \mathrm{~nm}) / \mathrm{Py}(5 \mathrm{~nm})$ trilayer is considered. However, as the results of qualitative study indicate the vortex in Py layer is extremely mobile and its displacement is significant. This might result in the appearance of the surface charges on the lateral sides of the computational area and, in turn, as discussed before it might have great impact on the frequency. Magnetostatic contribution from the boundary effect can not be corrected. However, to decrease the influence of artificial surface charges on the vortex dynamics, we have used a steeper attractive Oersted potential resulting in the vortex frequency increase and displacement confinement. For this purpose the Oersted field is calculated based on the infinite wire approximation but without the correction factor, 
on the contrary to the approach adapted in previous section. This procedure is advantageous from the practical point of view having minor consequences on the qualitative behavior of the system.

\subsubsection{Positive current}

For the electron flow from Co to Py layer the dynamics is observed as in previous section only in case of ST activated on Co layer. The remaining cases (ST-Py; ST-BL) yield no dynamic response which has been qualitatively explained before.

An interesting behavior is observed when ST is considered active only on Co layer. Both vortices, already at the threshold current of $0.5 \mathrm{~mA}$, are expelled away from the contact area. However, vortex in Co layer shows to be more mobile, i.e. its displacement is larger than the corresponding one in Py layer. An example of the orbit at $5 \mathrm{~mA}$ is presented in Fig. $5.8 \mathrm{~b}$, where black open squares correspond to Co vortex and red open circles to Py vortex trajectory. In the low current regime $(\mathrm{I}<9 \mathrm{~mA})$ each vortex moves $\mathrm{cw}$ with its own eigenfrequency $\left(\mathrm{f}_{\mathrm{Co}}\right.$ and $\mathrm{f}_{\mathrm{Py}}$ ) that exhibits blueshift with current, as presented in Fig. 5.8a. The temporal evolution of the average magnetization $\mathrm{x}$-component inside $\mathrm{PC}$ in $\mathrm{Co}$ (black) and $\mathrm{Py}$ (red) layer and the GMR signal amplitude (green) are presented in Fig. 5.9b together with the example spectra at $\mathrm{I}=4 \mathrm{~mA}$, Fig. 5.9a. The appearance of two distinct frequencies comes from the individual energy balance in Py and Co layer, owing to different material parameters and the presence of ST only in the latter. Also, what is going to be proven later, it is essential that both vortices remain outside the $\mathrm{PC}$ for two eigenfrequencies to be observed.

The direction of gyration of both vortices is cw and, therefore, the resulting GMR signal represents the difference of these eigenfrequencies $f_{\mathrm{Co}}-f_{\mathrm{Py}}$. The amplitude of the GMR signal decreases with current because both orbits 


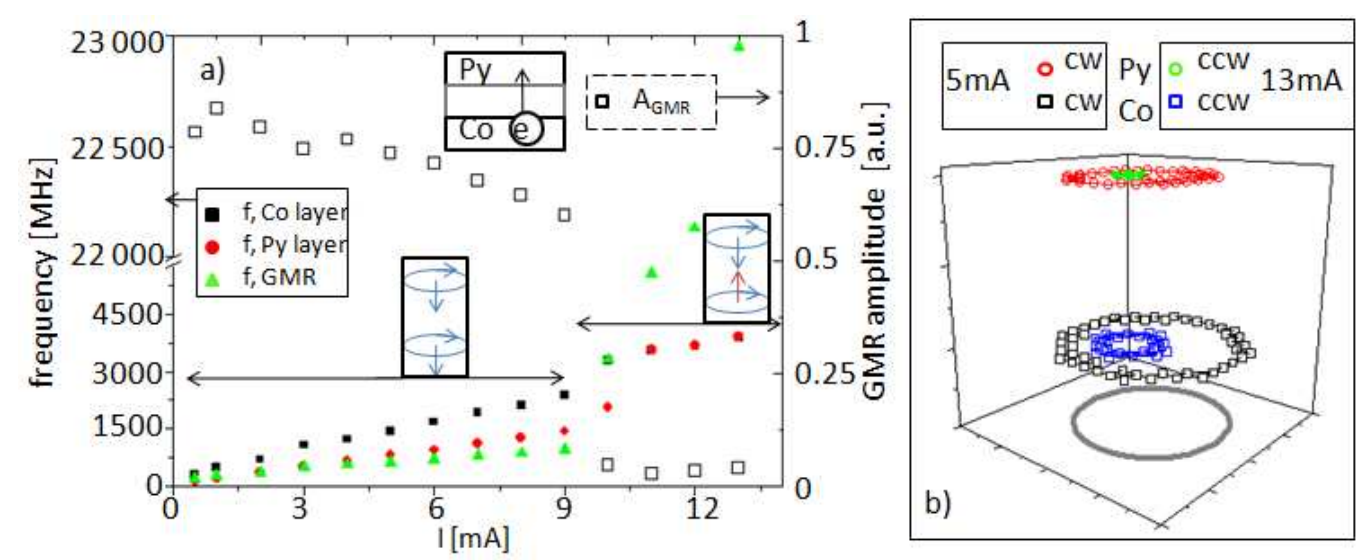

Fig. 5.8: Positive current, ST active only on Co layer. Frequency as function of current for the readout inside the $\mathrm{PC}$ in Co layer $\mathrm{f}_{\mathrm{Co}}$ (black), Py layer $\mathrm{f}_{\mathrm{Py}}$ (red) and extracted GMR signal $\mathrm{f}_{\mathrm{GMR}}$ (green). The latter exhibits frequency that corresponds to $\mathrm{f}_{\mathrm{Co}}-\mathrm{f}_{\mathrm{Py}}$ up to the critical current of $10 \mathrm{~mA}$ where Co vortex core changes the polarity. Right vertical axis: the amplitude of the GMR signal as function of current with the jump indicating qualitative change in the behavior of vortices, (a). Orbits of vortices gyrating in Py layer and Co layer at $5 \mathrm{~mA}$ both $\mathrm{cw}$ in agreement with right hand rule (red open circle, black open square, respectively) and $13 \mathrm{~mA}$ both ccw because positively charged vortex core in Co layer forces magnetostatically ccw movement of negatively charged Py vortex core (green open circle, blue open square, respectively), PC area is marked grey (b).

shrink as the current is increased. Since there is no ST acting on Py layer, the energy sustaining oscillations comes from the temporal evolution of the magnetostatic field generated by the moving vortex core in the Co layer. The last one, owing ST effect, increases its velocity, thus, providing steeper potential well for the oscillator in Py layer, which is why its displacement decreases with current.

At $10 \mathrm{~mA}$ a qualitative change in the behavior is observed. The resulting GMR signal is no longer the difference of two frequencies but it reflects 
solely the Co eigenfrequency and Py vortex decays to oscillate within PC area. This change is the consequence of vortex core switching in Co layer. Thus, vortex in Co layer reaches the critical velocity and as the core switches the direction of gyration is changed, i.e. it orbits the center outside the PC area in a ccw manner. At the same time vortex in Py, which preserves its negative polarity, decays to oscillate with minor amplitude very close to the $\mathrm{PC}$ center. Interestingly, it first continues to gyrate in cw direction but its movement becomes complicated to, finally, after some tens of nanoseconds begin to move ccw, which is against the right hand rule. In general, one would expect $\mathrm{cw}$ gyration of vortex to be preserved as the core remains polarized in the negative direction. Hence, it is the magnetostatic interaction between opposite charged cores of vortices in Py (negative) and Co layer (positive after core switch) that forces the first one to move ccw, following the direction of the ST driven motion of the second. This results in the downward jump in the associated GMR amplitude Fig. 5.8a.

Finally, in the high current regime, I $>10 \mathrm{~mA}$, GMR frequency completely decouples from the eigenfrequencies observed before but the oscillators remain synchronized to a common frequency. The GMR frequency spectrum becomes rich in higher harmonics in extremely high range (above $22 \mathrm{GHz}$ ) and it can not be related easily to the eigenfrequency of the synchronized oscillators. Meanwhile, the displacement of vortices in Co and Py layers decreases as indicated in Fig. 5.8b (13 mA) and both oscillate in ccw direction inside the PC.

Above this critical current, I $>13 \mathrm{~mA}$, multiple high frequency peaks appear in the GMR spectra, while the amplitude of the signal becomes negligible. The Co vortex undergoes successive core polarity switching below PC area. Each switching event is followed by the change of the direction of the low amplitude gyration and the emission of spin waves. On the other hand, vortex in Py layer preserves its negative polarity and oscillates $\mathrm{cw}$ within the $\mathrm{PC}$ area in a complicated manner accompanied by the spin wave nucleation. 
Thus, this movement can not be easily represented as a superposition of well defined low/high frequency components. Therefore, because it is beyond the interest of this work, no further study is performed for currents above $16 \mathrm{~mA}$.
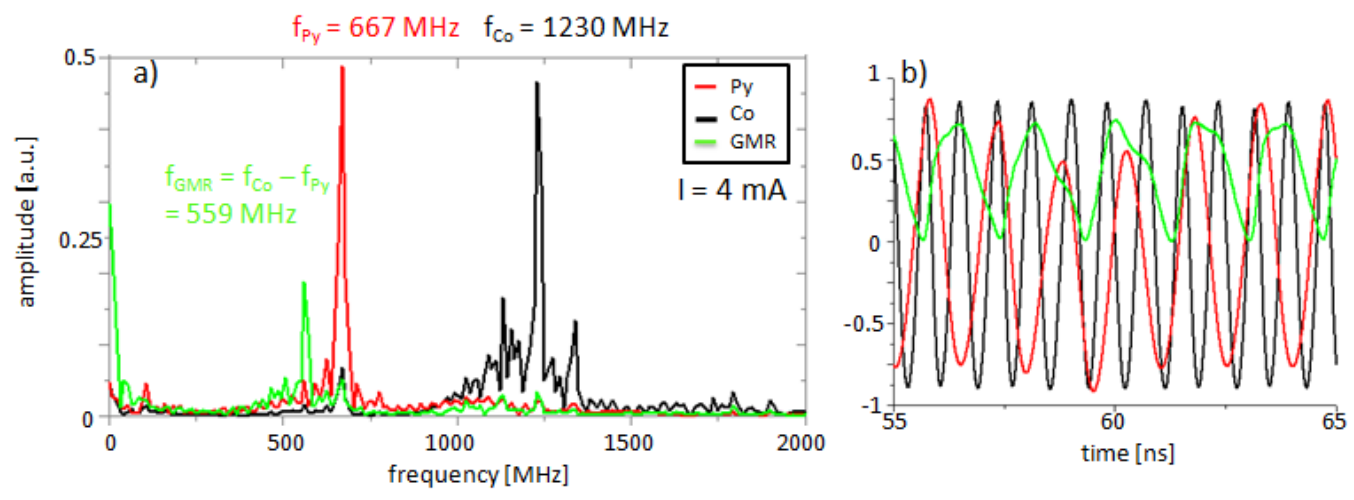

Fig. 5.9: Torque active only on Co layer, I $=4 \mathrm{~mA}$. Frequency spectra for the readout inside the PC in Co layer (black), Py layer (red) and resulting GMR (green), (a). Temporal evolution of average magnetization $\mathrm{x}$-component and GMR signal that served for calculation of the spectra presented, (b). The same color legend for both subfigures.

Note that even though ST is active only on the ferromagnet of higher saturation magnetization both vortices move with separate frequencies as long as the cores are of common polarity. Once the core of Co vortex has switched only one frequency was reported while the vortices decayed to move below the PC. Moreover, the coupling of the oscillator of lower saturation magnetization Py layer is strong enough to force its ccw movement despite the negative core polarity.

\subsubsection{Negative current}

At the negative current, the oscillations are observed as previously in cases of ST-Py or ST-BL. However, on the contrary to results of qualitative study 
with thicker Co layer, no output is reported when activating ST only on Co layer (ST-Co). Some very minor movements of vortices inside the PC are observed at low currents $(-3 \mathrm{~mA})$ but the displacement does not exceed few nanometers and it decreases with current. Moreover, the output GMR signal is negligible throughout studied range (down to $-10 \mathrm{~mA}$ ) and, thus, ST-Co is considered as yielding no dynamic output.

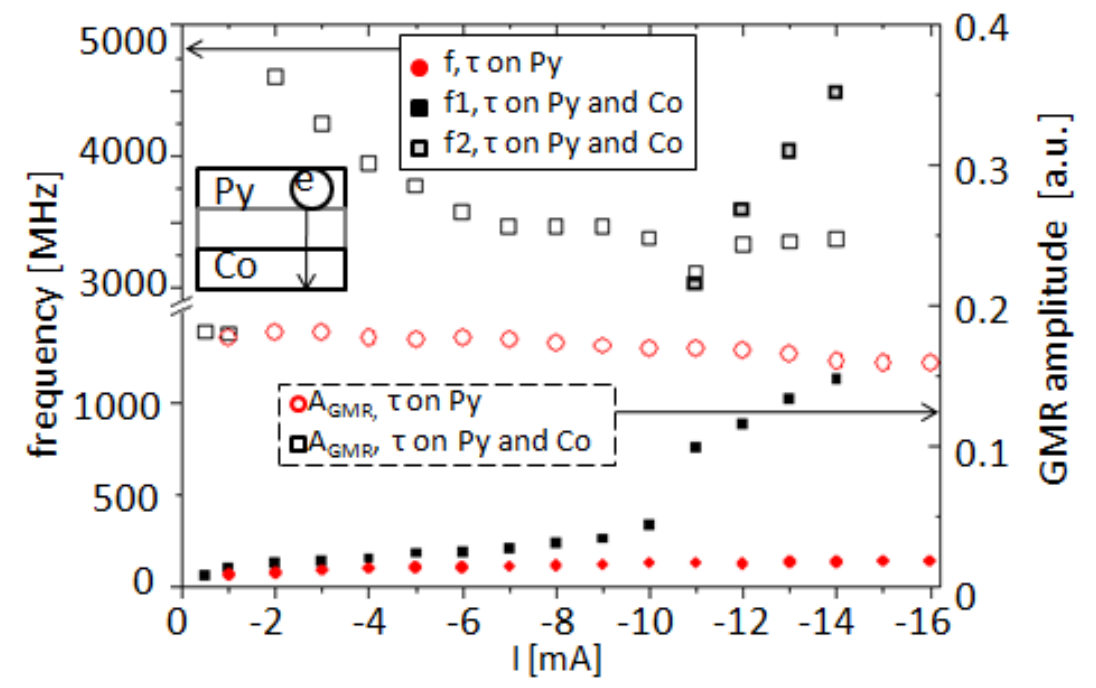

Fig. 5.10: Negative current. ST active on Py (full red circles) and simultaneously on Py and Co (full black squares, black squares filled grey). Left axis: frequency as function of current. Right axis: GMR signal amplitude as function of current (open symbols). If torque is activated only on Py layer the frequency increases and the amplitude of GMR signal decreases with current. If torque is activated on both layers more complicated behavior is observed. For details see text.

On the other hand very rich dynamics is observed in the remaining cases. In general, more mobile Py vortex is shifted to a well defined orbit outside 
the PC whereas Co vortex remains inside it. Interestingly, its low amplitude movement is synchronized with the eigenfrequency of Py vortex and the oscillators remain locked throughout the whole studied current range. On the contrary to results from previous section (positive current, ST-Co), no trace of two separate eigenfrequencies is found. Thus, in previously discussed in the current range where two frequencies were reported (and the GMR was shown to be their difference), both vortices moved in common direction and both were expelled from the PC area. As soon as one of the vortices (in Py layer) decayed to the inside of the PC (at $10 \mathrm{~mA}$ ) only one eigenfrequency was reported despite the common gyration direction (which inversed for both vortices).

Thus, in the case studied in this section (negative current, ST-Py) the appearance of only one frequency is to be expected if Co vortex remains inside the PC and two frequencies if Co vortex is expelled from the PC. Note that, two frequencies were reported when assuming ST effect active on a ferromagnet of high saturation magnetization (Co, positive current) and, as will be shown below, only one is found when ST active layer is of low saturation magnetization (Py, negative current). The reason is simple. It is easier to destabilize and expel a vortex in a material characterized by low saturation magnetization where demagnetizing effects are weaker. Additionally, certain magnetostatic core-core interactions are present. Hence, if Co vortex moves driven by the ST, by means of magnetostatic interaction it favors movement of vortex in Py layer and the latter responds, see Fig. 5.8b at $5 \mathrm{~mA}$. On the other hand, when Py is driven into motion by ST effect, it is displaced much further from the PC center, Fig. 5.11b. Thus, the core-core interaction, which scales with the distance, is then not sufficient to force the movement of vortex in Co layer where stronger demagnetizing effect are present and, therefore, the vortex in the latter remains inside the PC. This, in turn, leads to the situation where only one frequency can be reported. 
Even though, the displacement of Co vortex is larger in ST-BL than in $\mathrm{ST}-\mathrm{Py}$, which is attributed to the additional ST term in the energy balance in Co layer in the first, the Co vortex remains inside the PC in ST-BL. Thus, in this case, as will be shown, the magnetostatic interaction is not strong enough to expel the Co vortex outside the PC but its impact on the overall dynamics manifests itself in the introduction of important higher harmonics.

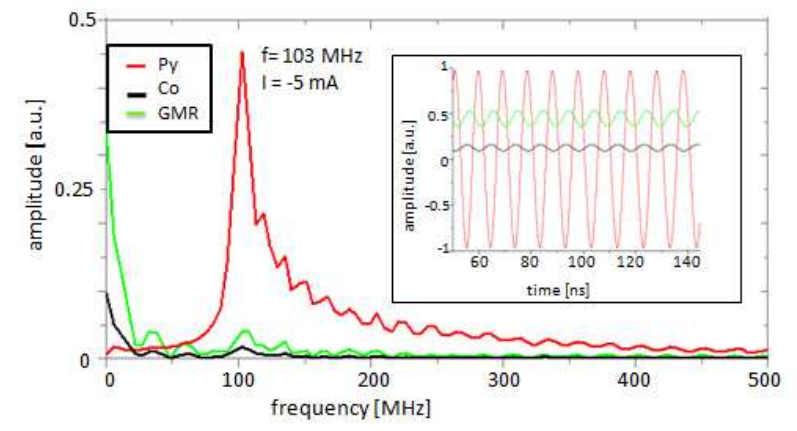

(a)

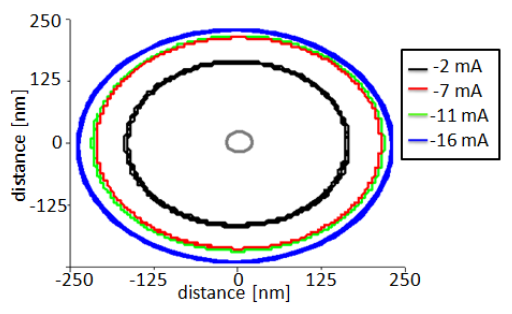

(b)

Fig. 5.11: Negative current, ST on Py layer. Frequency spectra for readouts at the PC in Co layer (black), Py layer (red) and resulting GMR (green), all exhibiting the same frequency of $103 \mathrm{MHz}$ at $\mathrm{I}=-5 \mathrm{~mA}$. Temporal evolution of average magnetization $\mathrm{x}$-component and GMR signal that served for calculation of the spectra presented in the inset, (a). The orbit of the vortex gyrating $\mathrm{cw}$ in Py layer at different currents. As the (absolute value of) current is increased $(|I| \uparrow)$ the corresponding displacement of the vortex increses, (b).

In ST-Py case one observes frequency nonlinear blueshift with absolute current value as indicated with full red circles in Fig. 5.10. The oscillations are observed at relatively low threshold of $-1 \mathrm{~mA}$ and continue until the critical current of $-16 \mathrm{~mA}$. Moreover, the highest output signal is present at the threshold current and later it decreases as the current is decreased. Very minor cw movements of vortex inside PC in Co layer yield little amplitude change in the temporal evolution of the corresponding overall average 


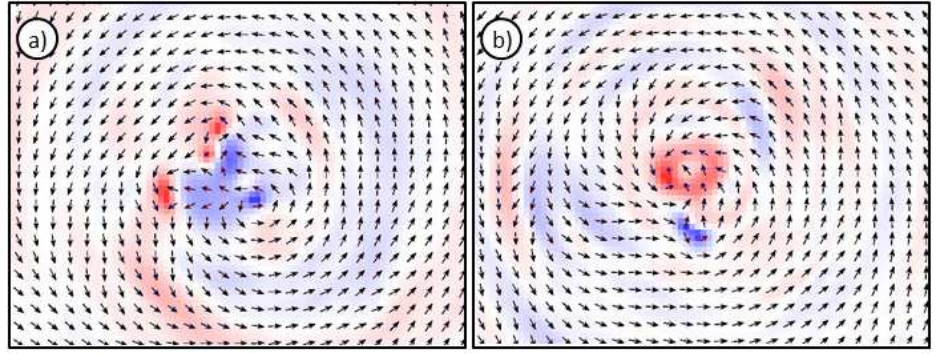

Fig. 5.12: Snapshots of the magnetization evolution in Py layer at different time instants. In color the $\mathrm{z}^{-}$-component. Vortex core polarity charges periodically, vortex-antivortex creation and expulsion and the spin wave radiation is reported.

magnetization as indicated in (black) inset in Fig. 5.11a. On the other hand, vortex in Py layer is expelled far from the $\mathrm{PC}$ which is reflected in the maximal change of the average magnetization $\mathrm{x}-$ component in the $\mathrm{PC}$ as indicated in (red) inset in Fig. 5.11a. This displacement shows to be function of current, so that the vortex orbit expands as the absolute current value is increased, Fig. 5.11b. Note, that the simulations are carried out for a finite structure, where the existence of computational boundaries introduces magnetic charges once vortex is destabilized from the central position.

The dependence of th displacement on current confirms that in finite structures ST influences the amplitude but not the frequency of the oscillations which is compatible with Ref. [41]. Moreover, since no ST is activated on Co layer the mechanism driving its cw movement is the magnetostatic coupling to the $\mathrm{cw}$ moving Py vortex. However, the movement of the first one remains minor because the magnetostatic interaction with far displaced vortex core in Py is not strong enough to expel the vortex core in Co layer where strong demagnetizing effects favor vortex central position. Therefore, up to the critical current only one frequency is reported. 


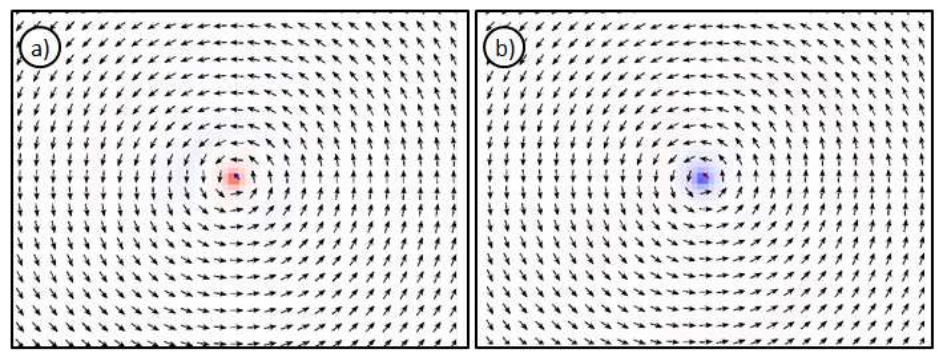

Fig. 5.13: Snapshots of the magnetization evolution in Co layer at different time instants. In color the $\mathrm{z}$-component. Vortex core polarity charges periodically but no vortex-antivortex creation is reported.

After crossing the critical point $(-16 \mathrm{~mA})$ vortex in Py layer, under ST influence, reaches the critical velocity and decays to the central position. It remains there undergoing ST-induced irregular switching of polarities which is followed by the nucleation and outward propagation of the spin waves and creation and expulsion of vortex-antivortex ( $\mathrm{V}-\mathrm{AV})$ pairs as presented in snapshots in Fig. 5.12. This influences the state of vortex in Co layer whose centrally situated core also undergoes periodic switching of the polarity but without $\mathrm{V}-\mathrm{AV}$ nucleation, Fig. 5.13. Thus, the demagnetizing effects in Co layer prevent its vortex from evolving into complicated dynamics. The processes of $\mathrm{V}-\mathrm{AV}$ nucleation and spin wave radiation are highly complex and are followed by the decrease of the output GMR signal and broadening of its spectrum. The subject of this chapter is the characterization of oscillations of two well defined vortices and, therefore, no further study is carried out below current of $-18 \mathrm{~mA}$.

On the other hand, in ST-BL the dynamics becomes more complicated due to the presence of two competitive torques. In general, frequency blueshift with absolute current is observed as presented in Fig. 5.10 with black full squares. However, two distinct regimes are observed. Down to the critical 
current of $-11 \mathrm{~mA}$ a slow, nonlinear frequency blueshift and the associated GMR signal amplitude decrease with current (black open squares, Fig. 5.10) are reported. Moreover only one eigenfrequency is present i.e. one maximum is found in the data extracted from PC in Py, Co layers and the associated GMR data as shown in Fig. 5.14a. Even though the oscillators remain synchronized to one frequency down to the critical current the presence of two distinct torques manifests itself clearly already below $-2 \mathrm{~mA}$. In order to explain it in detail reconsider Fig. 5.10 with respect to GMR signal amplitude evolution with current as presented in Fig. 5.16. The differences arising from the addition of the ST on Co layer are to be extracted by comparing $\mathrm{ST}-\mathrm{BL}$ to ST-Py.

At, and close to, the threshold current similar qualitative and quantitative result is observed. Note that at negative current, as long as in Py layer the vortex remains in the $\mathrm{PC}$ center the $\mathrm{ST}$ acting on Co is stabilizing its vortex in the initial central position. Above that, recalling that ST magnitude is inversly proportional to the saturation magnetization of the layer, the ST is almost three times stronger in Py as compared to Co layer. Moreover, from the experiments we know that there exists a threshold current for the STdriven vortex dynamics. This threshold is larger in Co layer where stronger demagnetizing effects are present. Therefore, even though ST is present in both layers, Co layer responds first at higher currents. Thus, the only effectively acting torque is the one on Py layer $\left(\tau_{\mathrm{Py}} \gg \tau_{\mathrm{Co}}\right)$ and results resembling ST-Py at $|\mathrm{I}|<2 \mathrm{~mA}$ are obtained.

As the absolute current is increased the displacement of vortex in Co layer becomes larger in ST-BL than in ST-Py as the first one accounts for the ST contribution in Co layer additionally supporting vortex destabilization in Co layer (once vortex in Py is destabilized). Still, ST-Py and ST-BL yield picture of very low amplitude Co vortex oscillations within the PC. Thus, for currents $2 \mathrm{~mA} \leq|\mathrm{I}| \leq 6 \mathrm{~mA}$ torque acting on Py remains dominating $\tau_{\mathrm{Py}}>\tau_{\mathrm{Co}}$ and one reports qualitatively similar result: GMR amplitude 


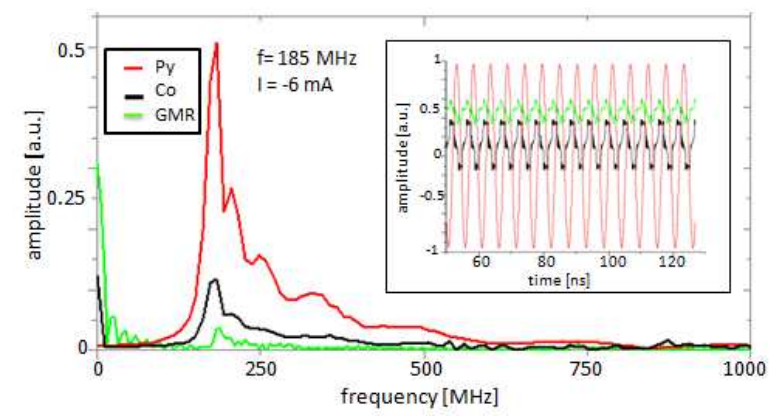

(a)

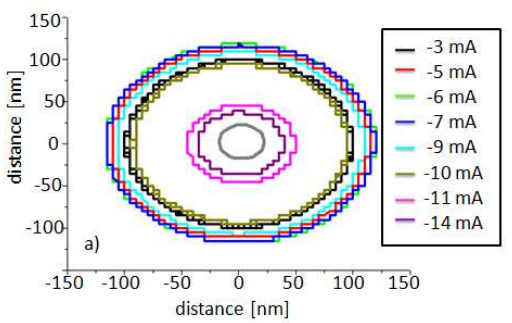

(b)

Fig. 5.14: Negative current, ST on both layers. Frequency spectra for PC readout in Co layer (black), Py layer (red) and resulting GMR (green), all marking the same frequency of $185 \mathrm{MHz}$ at $\mathrm{I}=-6 \mathrm{~mA}$. Temporal evolution of average magnetization $\mathrm{x}$-component and GMR signal that served for calculation of the spectra presented in the inset, (a). The orbit of the vortex gyrating $\mathrm{cw}$ in Py layer for different currents. As the (absolute value of) current is increased $(|I| \uparrow)$ the corresponding displacement of the vortex first increases, $\mathrm{I}>-6 \mathrm{~mA}$, then stabilizes, $-6 \mathrm{~mA}>\mathrm{I}>-10 \mathrm{~mA}$, and finally decreases, $\mathrm{I}<-10 \mathrm{~mA}$, (b).

decreases with absolute current, Fig. 5.16, and Py vortex orbit expands, Fig. 5.14b (compare at $-3 \mathrm{~mA}$ and $-5 \mathrm{~mA}$ ). The remaining quantitative difference in frequencies and corresponding GMR amplitude arises from the larger displacement of Co vortex and its minor but detectable movement in ST-BL.

At larger currents $7 \mathrm{~mA} \leq|\mathrm{I}| \leq 10 \mathrm{~mA}$ the ST threshold in Co layer is reached i.e. the effective activation of ST-driven dynamics in this layer is achieved. This favors alignment of Co vortex core along the axis of Py vortex - an effect which is additionally strengthened by the magnetostatic core-core interaction. As a result Py vortex orbit is (in a short current range) almost 


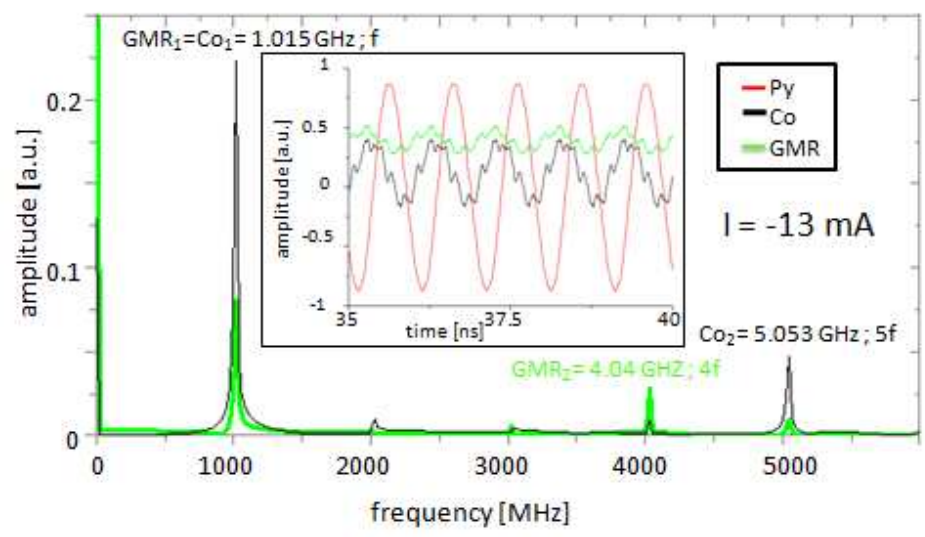

Fig. 5.15: The frequency spectrum at $-13 \mathrm{~mA}$ revealing the existence of two distinct peaks in Co (black) and GMR (green) spectra. Inset presents the temporal evolution of the average magnetization $\mathrm{x}$-component inside the PC in Py (red), Co (black) layers and the corresponding GMR signal (green).

independent of current, Fig. 5.14b (compare $-7 \mathrm{~mA},-9 \mathrm{~mA}$ ) which, in turn, stabilizes the GMR signal amplitude, Fig. 5.16.

Recall that at negative current no dynamics was observed in ST-Co case because electrons polarized in Py acted stabilizing on Co layer configuration. On the other hand, considering ST-BL, the competition between the destabilizing (on low $\mathrm{M}_{\mathrm{s}}$ ferromagnet) and stabilizing (on high $\mathrm{M}_{\mathrm{s}}$ ferromagnet) torque is observed providing the initial trigger for the dynamics to be observed by destabilizing the first at low currents. Hence, the activation of ST-driven vortex dynamics in Co layer is possible first at higher current since ST response threshold in high $\mathrm{M}_{\mathrm{s}}$ Co layer is higher than in a low $\mathrm{M}_{\mathrm{s}}$ Py layer. Thus, in this range the dynamics is driven by the superposition of both torques effectively active $\left(\tau_{\mathrm{Py}} \approx \tau_{\mathrm{Co}}\right)$ and the magnetosatic interaction between the cores. The velocity of vortices increases which translates into steeper profiles of potential wells arising from the time derivative of magnetization configuration in each layer. Therefore, the expansion of Py vortex 
orbit is hindered and the GMR amplitude reaches minimum.

At the critical current of $-11 \mathrm{~mA}$ an upward jump in frequency is reported followed by a fast linear blueshift with current. An associated downward jump in the GMR amplitude, Fig. 5.16, and the appearance of the second peak in the GMR spectrum, Fig. 5.15, are identified. Moreover, Py vortex orbit starts shrinking, compare $-11 \mathrm{~mA}$ and $-14 \mathrm{~mA}$ in Fig. 5.14b. This qualitative change is traced back to the appearance of the second peak $\left(G_{2 M R}\right)$ in the output signal spectra corresponding to the four times the base frequency, 4f, as presented in Fig. 5.15. This is the consequence of compromise between the ST activation on Co layer and magnetostatic interactions, i.e. vortex in Co layer moves as a superposition of two distinct frequencies: low base frequency, f, of the cw movement and high frequency, $5 \mathrm{f}$, of the $\mathrm{cw}$ movement imposed by the magnetostatic interaction between the cores. Thus, combined with the cw low frequency movement of Py vortex one obtains read out frequency of $\mathrm{GMR}_{2}=5 \mathrm{f}-\mathrm{f}=4 \mathrm{f}$.

Both frequencies (f, 4f) are observed down to the critical current of $-14 \mathrm{~mA}$ below which the vortices reach the critical velocity and the successive polarity switching is observed. In Co layer the vortex tends to switch polarity and remains in the new state oscillating outside $\mathrm{PC}$ in a nanosecond time windows (before next core switching event). The polarity of the vortex defines the direction of vortex gyration according to right hand rule so that core switching is followed by the change of gyration direction. In Py layer the vortex decays to central position and undergoes fast core switching followed by the spin wave radiation. Study of this current range is beyond interest of this work.

\subsubsection{Results summary}

Note that the strong influence of the Oersted field imposes the same chirality of the vortices but the core polarity could, at least in principle, depend 
on a complicated dynamical nucleation process. Therefore, situation where vortices exhibit opposite core polarities could also be a possible initial state. Thus, not only the ST term but also the magnetodipolar interaction term (magnetostatic contribution from neighboring layer) in the considered trilayer are modified which should clearly have impact on the induced dynamics. Hence, it is interesting to carry out qualitative and quantitative study in order to define the range in which the frequency can be tuned by simple flip of the core polarity.

In the following the ST contribution shall be divided into two parts which are hereafter referred to as the in-plane and out-of-plane ST. Recall, that as introduced in Section 5.1.3 and up to now the discussion was carried out only with respect to the IP component. Hence, in the following both are to be used for argumentation. The first one (IP ST) corresponds to the acquired IP polarization of electrons defined by the vortex chirality and the second, analogous, to the vortex polarity (OP ST) in the polarizing layer.

Thus, to get a different view of the results already presented, which will allow easier comparison to the new results, it is convenient to summarize them in form of a schematic chart as presented in Fig. 5.17. Observations gathered up to now are presented in column I, where two vortices of the same chirality and polarity were considered. All studied cases are separated by defining the ST active layer, marked grey (top, bottom, both), and the corresponding ferromagnets F1 and F2 (either Co or Py). This summary provides a clear overview of the cases where dynamic response was observed.

First a brief summary of the comparison between observations emerging from I and II is to be carried out and later the new findings are presented in detail. The subfigures (a)-(h) of Fig. 5.17 schematically show the investigated cases, marking with full corner triangle cases yielding dynamic output. In general, at the test current of $5 \mathrm{~mA}$ the dynamics in II is observed only when setting F1 to be Py and F2 to be Co layer, independent of the definition of the ST active layer (top, bottom, both layers), while in I the oscillations are 
reported only in some particular (difficult to group out) cases.

Lower saturation magnetization ferromagnet as active layer. Consider first Py layer to be ST active (target layer) as is the case in (a), (b), (g), (h). Note that dynamics, observed in (a) and (b), is induced by the reflected electrons which polarize with opposite chirality with respect to state in the target layer. Therefore, in both (a) and (b) the same destabilizing IP ST is present. Similarly, the same IP ST term (from transmitted electrons) is observed in $(\mathrm{g})$ and $(\mathrm{h})$ where the dynamics is hindered by its stabilizing effect. Moreover, in (h) no dynamics is observed even though the transmitted electrons carry the OP momentum opposite to the vortex polarity in the target layer acting, therefore, destabilizing. Presence (a) and absence (b) of this OP ST lead to some further differences in the dynamical response which shall be discussed in detail later.

Higher saturation magnetization ferromagnet as active layer. On the other hand, assuming Co to be active layer and comparing (c), (d), (e) and (f) one concludes that dynamics is observed only in presence on the OP ST and independent of the IP ST as in (c) (IP ST present) and (f) (IP ST absent). In (e) the electrons polarize exactly parallel to the state in the target and, therefore, no dynamics is observed, while in the remaining (d) even the presence of the IP ST does not induce any oscillations (at the test current). Thus, in order to extract more information (c) and (f) are to be compared systematically. If both layers are set active I (i), (k) and II (j), (l) yield similar results and the exact comparison is to be carried out in the following.

Above observations lead to the conclusion that in case low $\mathrm{M}_{\mathrm{s}}$ ferromagnets (Py) being active layer the existence of the destabilizing IP ST is enough for the oscillations to be sustained, while higher $\mathrm{M}_{\mathrm{s}}$ ferromagnet (Co) responds dynamically in presence of the OP ST. Note that in addition to the ST effect the magnetostatic interaction plays important role if the distance between the cores is small. Thus, in case of Py as active layer, the additional 
magnetostatic core-core interaction changes quantitatively response of the system, (a) and (b). On the other hand, in case of Co as active layer, that is less sensitive to ST because of higher $\mathrm{M}_{\mathrm{s}}$, but where strong demagnetizing effects are present, the additional magnetostatic contribution might lead to disappearance of dynamic response, compare (c) and (d).

\subsubsection{Two vortices of different polarity}

In the following a detailed qualitative and quantitative discussion of the results highlighted above is presented. This study, depending on the definition of the ST active layer: Py, Co, both, provides a full comparison between the result emerging when employing different initial states as presented in Fig. 5.17. Hence, considering Py to be active layer (a) and (b) are to be compared, analogous, (c) and (f) when setting Co to be the free layer. Finally, both layers are allowed to evolve dynamically upon the ST effect and results arising from I and II are compared, (i) and (j).

Note that when assuming vortices of different polarities (column II in Fig. 5.17) the dynamics is observed independent of the definition of ST layer in the sample but as long as Py is set the top ferromagnetic layer, (b), (f), (j). Interestingly, the comparison of frequency evolution with (absolute) current in these three cases, as presented in Fig. 5.18a, points out common quantitative results, i.e. the frequency values are similar. On the other hand, the amplitude of the GMR signal, Fig. 5.18b, indicates the existence of some qualitative differences between the observed oscillations. In general, at high currents the vortex in Py layer reaches critical velocity and switches its polarity yielding back the situation of two vortices of equal chirality and polarity. The following sections proceed with the quantitative study. 
ST active on Py layer

ST-Py is studied first and comparison between cases yielding dynamic output, (a) and (c) in Fig. 5.17, is carried out. Note that results of (a) were presented in Figs. 5.10 and 5.11, and are now to be compared to the results of (c) in Fig. 5.19. The current applied in both cases corresponds to electrons flowing flow Py to Co layer. Following similarities are observed:

- frequency blueshift with current

- GMR amplitude decrease with current

- extremely low vortex mobility in Co layer

- existence of one sole eigenfrequency in the GMR signal

- no higher harmonics in the GMR spectra,

and the differences as comparing (c) to (a)

- steeper slope of the frequency blueshift with current

- much higher frequency values reaching an order of magnitude quantitative difference at the critical current

- significantly smaller vortex displacement in Py layer (30 nm as compared to $250 \mathrm{~nm}$ )

- current independent orbit of the vortex in Py layer (as compared to orbit expansion with current).

The reason for the appearance of the above mentioned differences is twofold. The change of the vortex core polarity in the polarizing Co layer not only affects the profile of the ST but also the magnetostatic field in the 
trilayer. Two vortices act as two magnetic dipoles and, therefore, the movement of one tend to force the movement of the other. The character of this interaction is defined by the relative orientation of the cores.

Note that even though the ST term is directly proportional to the applied current, the relative displacement of the vortex core in Py layer is found to be current independent, Fig. 5.19b. At the same time vortex core in Co layer undergoes ccw circular motion of minor amplitude $(5 \mathrm{~nm}$ away from the PC center). Thus, small distance between the cores in neighboring layers implies that core-core magnetostatic interaction could be of importance in defining quantitatively the gyration frequency. Moreover, qualitatively the minor ccw motion of vortex in Co layer arises from the coupling to the ccw movement of vortex in Py layer. Otherwise it would move cw owing its negative polarity.

At the current of $-14 \mathrm{~mA}$ the vortex in Py layer reaches critical velocity and its polarity is switched. This transition is followed by the reappearance of the mode observed in Fig. 5.10, i.e. vortices of the same polarity with all the consequences: expansion of the orbit into the one as in Fig. 5.11b, the corresponding jump in the GMR signal amplitude, critical current corresponding to the periodic switching of the core and spin wave emission as thoroughly discussed before.

Recently developed model [80] demonstrates that the current independent vortex orbit is a result of the balance between the dissipative Oersted field term and ST. These two terms represented by surface integrals and calculated numerically show to counterbalance each other just outside the PC when considering vortices of opposite polarity, which is in agreement with the micromagnetic simulations, Fig. 5.19b. For qualitative explanation of this effect see Fig. 5.20. The vortices are represented in black, while the direction of polarized electrons in red. Note that the IP ST acts destabilizing on the vortex while the OP ST favors central position of the core if the vortices are of opposite polarity. Thus, the resulting core displacement is a compromise between the two ST terms and the dissipative Oersted field effect. On the 
contrary, in the case of the same polarity the vortex is expelled far from the contact as both IP ST and OP ST destabilize its central position.

It is worth mentioning that minor vortex movements inside the $\mathrm{PC}$ in Co layer neither qualitatively nor quantitatively influence the dynamics in the adjacent Py layer i.e. no change in the outputs is observed even if the configuration in Co layer is assumed fixed in time. Thus, an interesting feature is observed if the magnetostatic field created by Co layer is neglected. In this case only the dynamics in Py layer is resolved while the configuration in Co serves only for the determination of the ST profile.

Consider the case of the same vortex polarity shown in left hand side of Fig. 5.21. Py layer study and trilayer study yield quantitatively the same results. This indicates that if the cores are far apart then the ST is the main factor determining the frequency and the displacement of the oscillating vortex. The second was to be expected as the strength of core-core magnetostatic interactions is inversly proportional to the distance between them so this term is negligible at significant separation.

On the other hand, if vortices remain close to each other (see the right hand side of Fig. 5.21), the core-core interactions might influence the frequency of the vortex oscillating in the free layer. Still, in absence of pining centers, defects and physical boundaries the orbit of the gyrating vortex is solely defined by the counterbalance between the ST and dissipation associated with the Oersted field.

Following conclusions can be drawn from the results presented above. ST and the dissipative Oersted field term are the factors determining the displacement of the vortex core in the free layer [80]. On the other hand the magnetostatic core-core interactions scale inversly proportional to this 
displacement so the frequency of the oscillating vortex is influenced quantitatively only if the intercore distance is small.

\section{ST active on Co layer}

Setting Co to be the ST active layer leads to observation of dynamic output in cases marked as (c) and (f) in Fig. 5.17. Note that according to the adapted current sign convention (positive current $=$ electron flow from $\mathrm{Co}$ to $\mathrm{Py}$ ) (c) represents positive current direction while (f) the negative one. Hence, assuming higher $\mathrm{M}_{\mathrm{s}}$ ferromagnetic layer to be subject to ST leads to sustained output for both current directions, depending on the relative polarity of the vortices. At positive current the dynamics is found for the same core polarization while at the negative in the opposite case as shown in Fig. 5.22.

For sake of simplicity the following explanation of behavior at negative current is carried out referring to its absolute value, Fig. 5.25. The oscillations start at the threshold of $0.5 \mathrm{~mA}$. Up to $6 \mathrm{~mA}$ the orbit of the vortex in Co layer is very confined, while Py vortex oscillates close to the PC. Both movements are ccw and the orbits are current independent in this range (compare $-2 \mathrm{~mA}$ and $-3 \mathrm{~mA}$ in Fig. 5.23b). Thus, ccw direction of Co vortex gyration is against the right hand rule and is a consequence of magnetostatic coupling to ccw movement of Py vortex. Only one eingenfrequency is visible, Fig. 5.23a, and the corresponding GMR amplitude increases with the absolute current. 


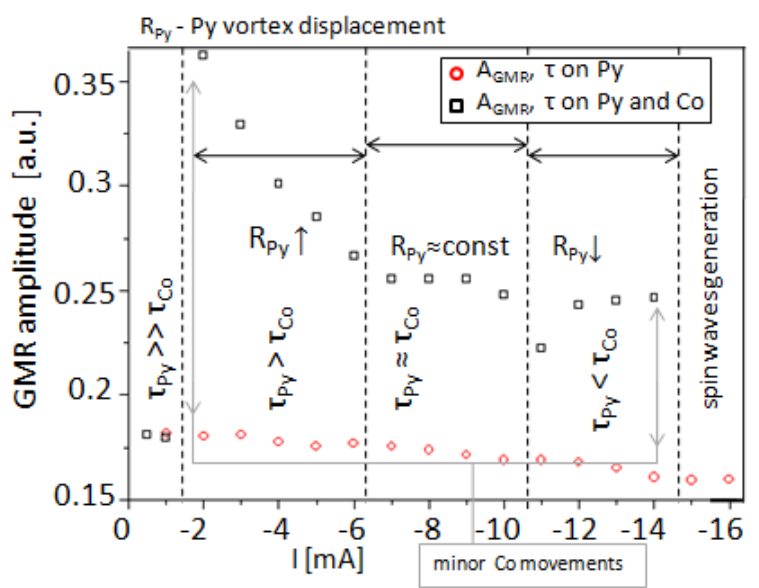

Fig. 5.16: Five qualitatively different current ranges where the initially effectively active ST on Py layer is gradually complemented with the ST effect on Co layer activating itself at higher current. Close to the threshold only ST on Py is effectively active, $\tau_{\mathrm{Py}} \gg \tau_{\mathrm{Co}}$, and it keeps dominating the dynamics at low currents leading to expansion of Py vortex orbit, $\tau_{\text {Py }}>\tau_{\text {Co }}$. The effective activation of ST on Co layer stabilizes the Py vortex on a fixed orbit resulting in the stabilization of the GMR amplitude $\tau_{\mathrm{Py}} \approx \tau_{\mathrm{Co}}$. Finally, interplay between the torque and magnetostatic interactions leads to shrinking of Py vortex orbit and the associated jump in the GMR amplitude, $\tau_{\text {Py }}<\tau_{\text {Co }}$. Below $-14 \mathrm{~mA}$ Py vortex reaches critical velocity for core reversal and both vortices undergo periodic polarity switching. The quantitative difference in the resulting GMR amplitude between ST-Py and ST-BL in the range between $-2 \mathrm{~mA}$ and $-14 \mathrm{~mA}$ arises from larger displacement of Co vortex in ST-BL. 

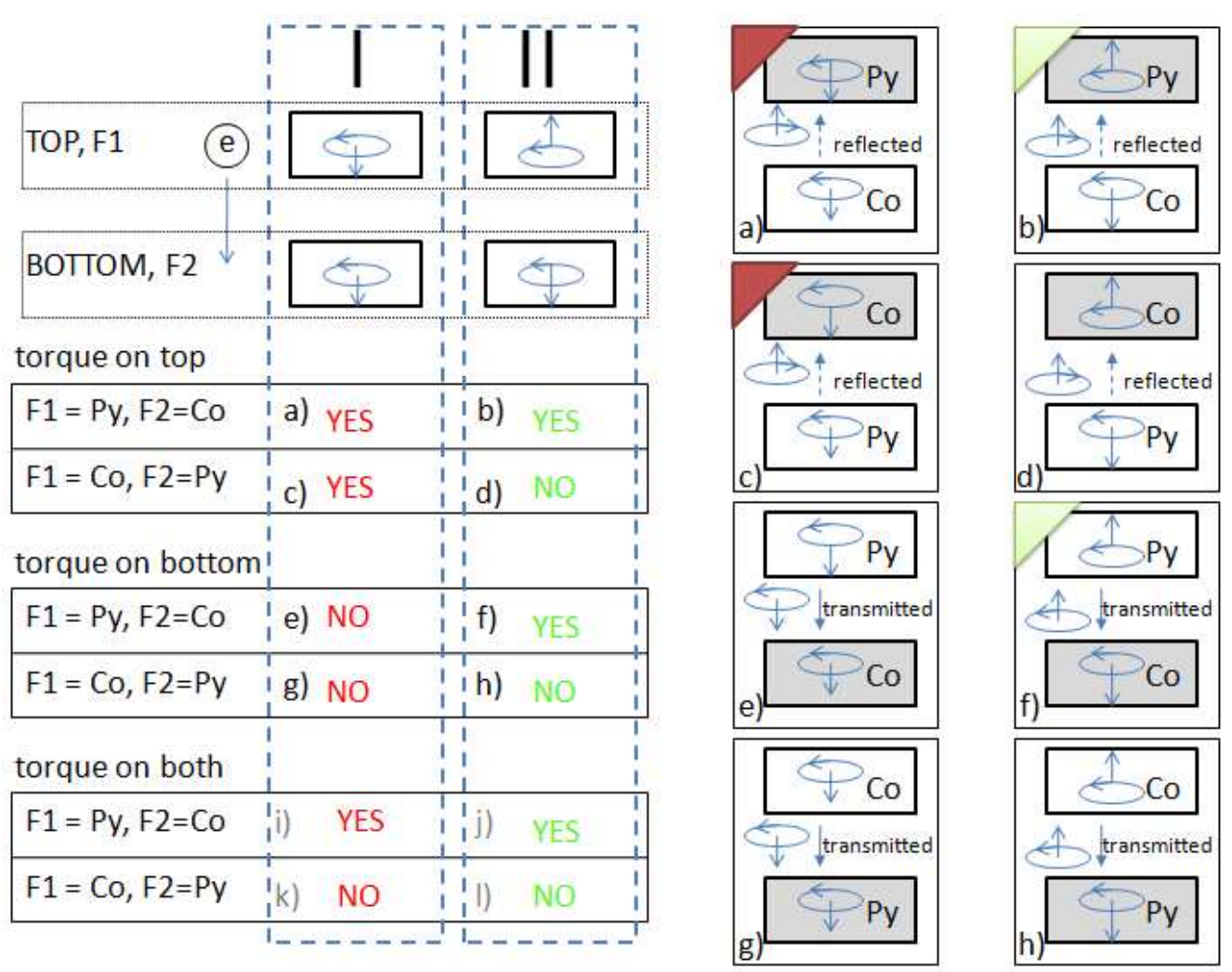

Fig. 5.17: Left hand side, scheme of the initial states corresponding to both vortices of the same chirality and polarity in I and opposite polarity in II. I: the results of previously discussed study ordered by activating the ST on each and both layers and alternating the material of the top and bottom layer (either Py or Co). Right hand side, schemes of the trilayer corresponding to cases (a) - (h). The initial states in the ferromagnetic layers and the state of the polarized electrons are presented. Depending on the current direction and the definition of the ST active layer the electrons are referred to as reflected or transmitted. Full corner triangles mark cases yielding dynamic output. 

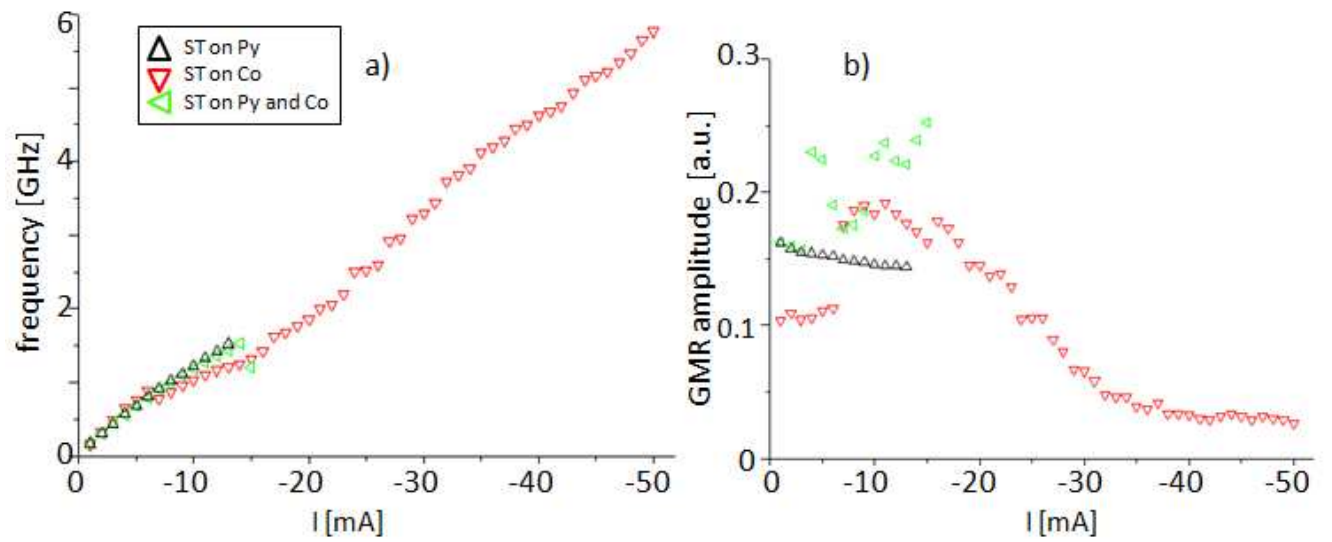

Fig. 5.18: Frequency evolution with current defining Py (black triangles), Co (red inverted triangles) and both layers (green left triangles) to be ST active, (a). The resulting frequency blueshift with absolute current yields quantitatively similar values in all cases. At high currents the polarity of Py vortex is switched. The amplitude of the GMR signal corresponding to the cases from (a), indicates qualitative differences in the oscillations observed, (b). 


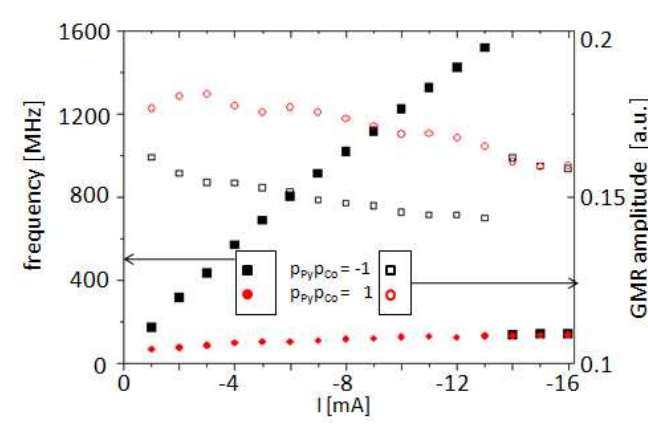

(a)

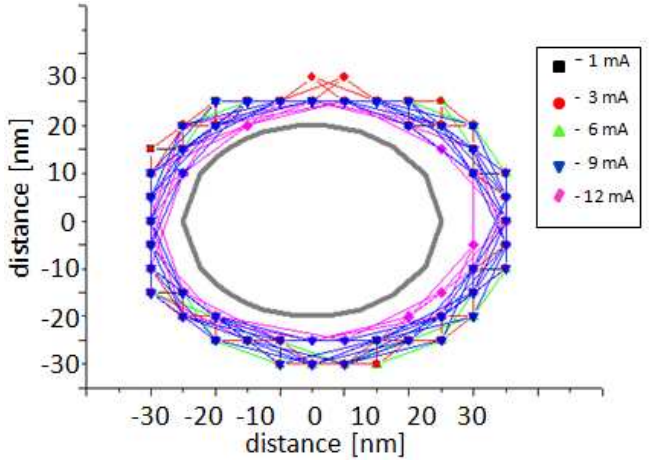

(b)

Fig. 5.19: Comparison of results of systematic study corresponding to Fig. 5.17a and Fig. 5.17b, Py set the ST active layer. Left axis: frequency evolution with current arising when considering vortices of common polarity, full red circles and of opposite polarity, full black squares; right axis corresponding GMR signal amplitude with open symbols, (a). Below $-13 \mathrm{~mA}$ the polarity of the vortex in the top Py layer switches. The current independent trajectory of the vortex moving close to the PC (grey) in Py layer, (b). Vortex in Co layer undergoes some minor oscillations in the PC center (not shown).

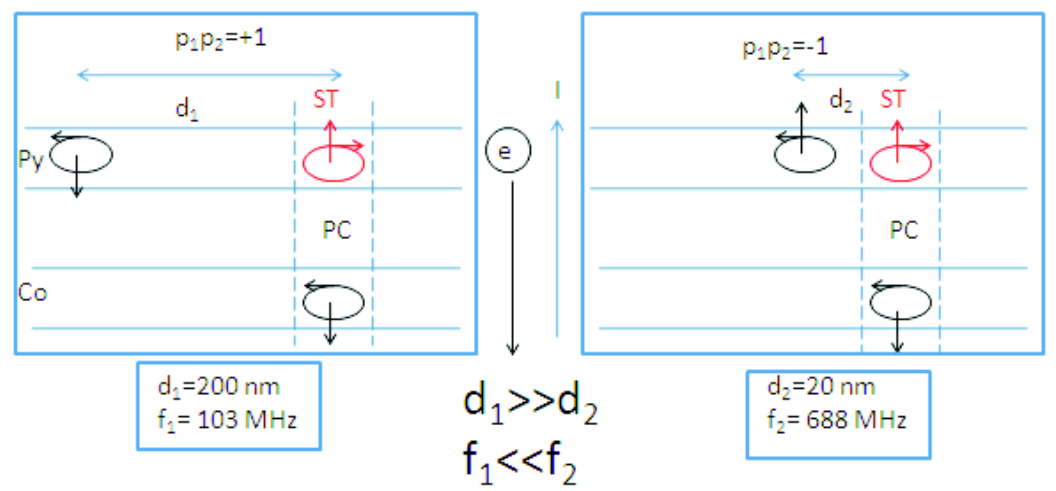

Fig. 5.20: Scheme of studied cases together with some quantitative results for comparison. Vortices are represented in black and the profile of the ST in red. 


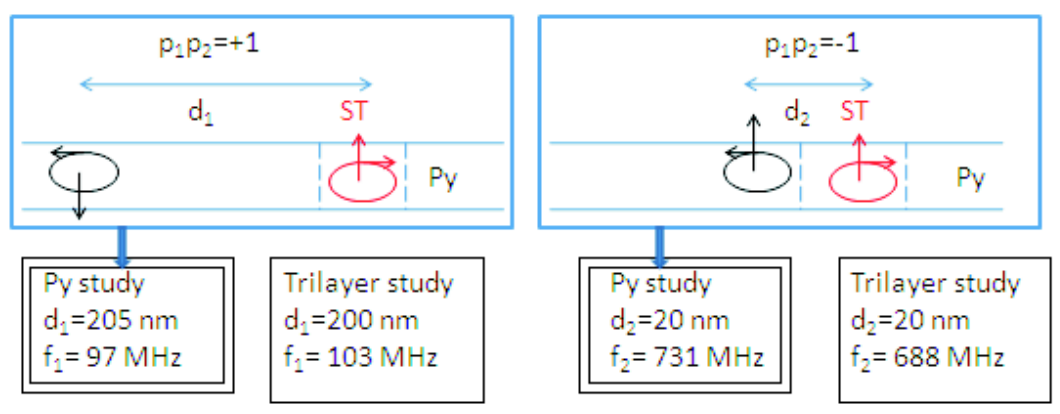

Fig. 5.21: Schematic representation of the results when only Py layer is simulated. Comparison to the trilayer study in numbers in the tables.

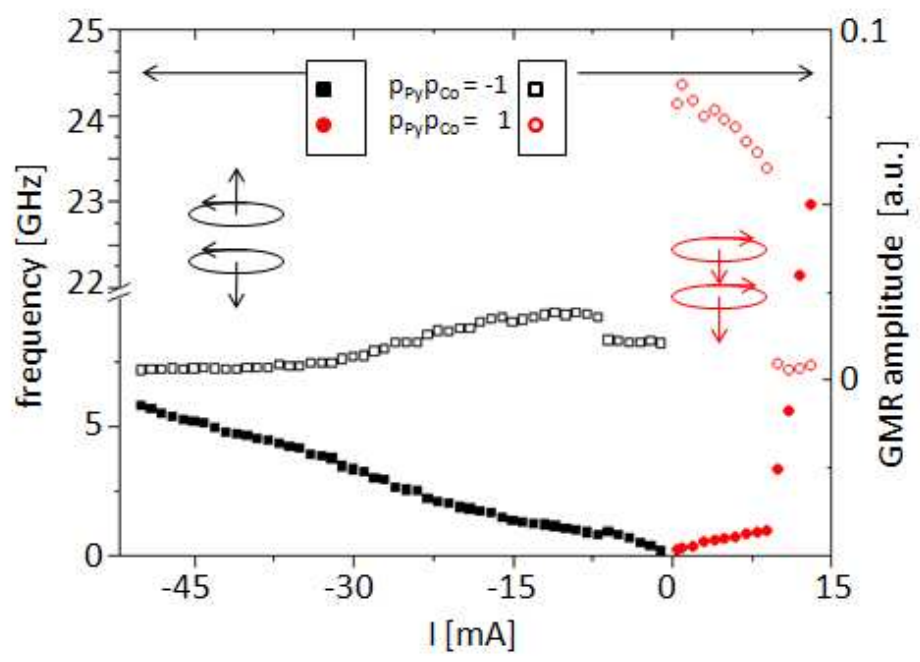

Fig. 5.22: ST-Co. At positive current the dynamics is found for a common core polarization while at the negative in the opposite case. 


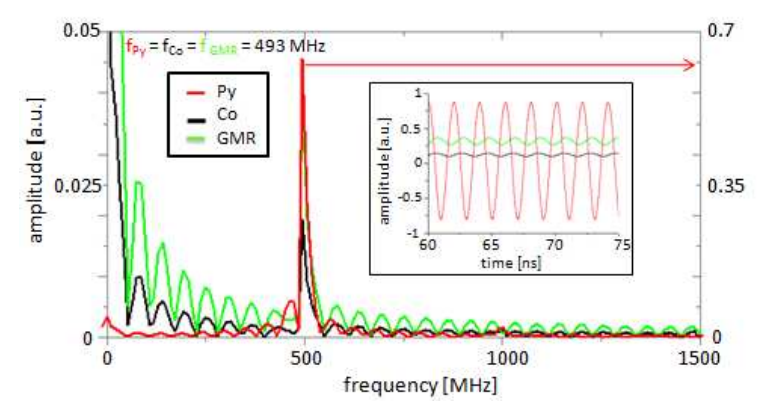

(a)

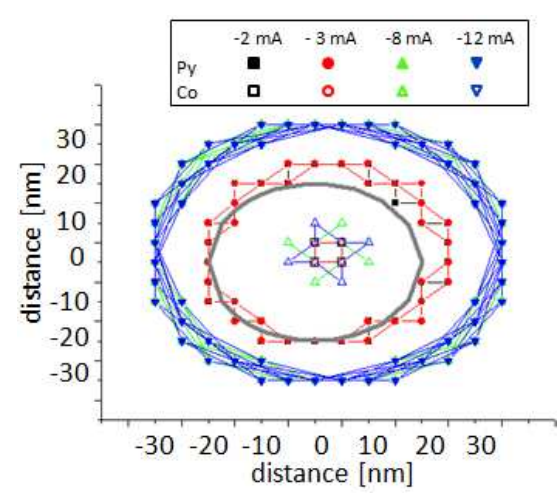

(b)

Fig. 5.23: Spectra extracted at the current of $-3 \mathrm{~mA}$ in $\mathrm{Py}$ (red, right vertical axis), Co (black, left vertical axis) layer and the resulting GMR signal (green, left vertical axis). The temporal evolution of the average magnetization $\mathrm{x}$-component in the $\mathrm{PC}$ and the associated GMR that served for the calculation of the spectra in the inset with the same color legend, (a). One eigenfrequency is observed in the system. The displacement of the vortices in Py and Co layer at different currents, (b).

At the absolute current of $7 \mathrm{~mA}$ the appearance of additional frequency is found. This is the consequence of reaching the threshold for ST-driven dynamics in Co layer. Thus, the existence of two frequencies in the GMR spectrum is associated to the complicated evolution of vortex position in Co layer which yields strong higher harmonics. Moreover, vortex orbit in Py expands (compare $-2 \mathrm{~mA}$ and $-8 \mathrm{~mA}$ in Fig. 5.23b) and an upward jump in the associated GMR amplitude is observed.

At the absolute current of $8 \mathrm{~mA}$ one more higher harmonic becomes visible. The two additional side peaks in the frequency spectrum are visible as 

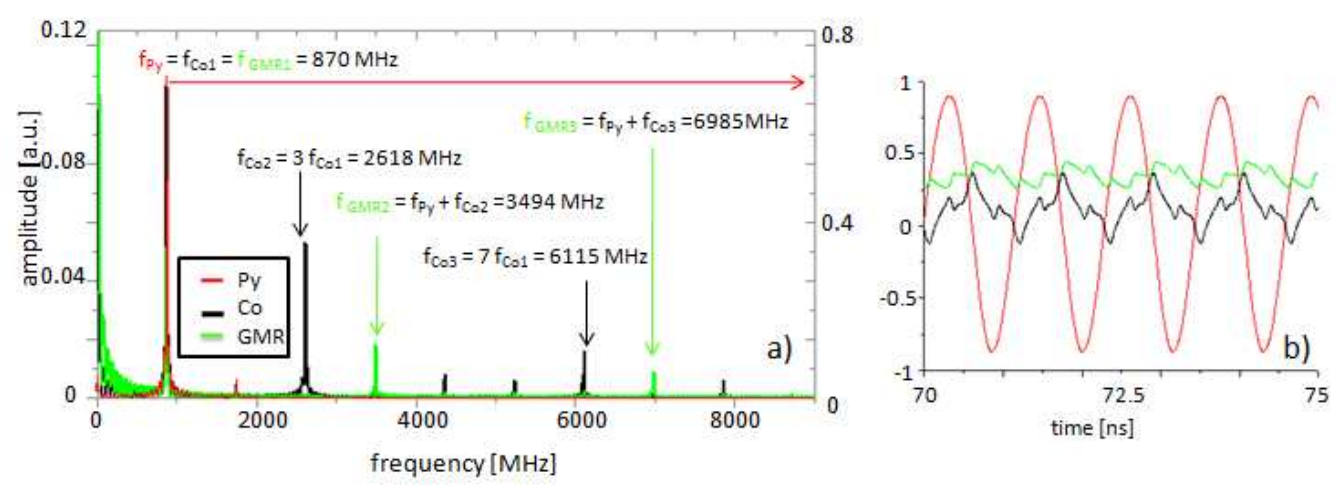

Fig. 5.24: Spectra extracted at the current of $-8 \mathrm{~mA}$ in $\mathrm{Py}$ (red, right vertical axis), Co (black, left vertical axis) layer and the resulting GMR signal (green, left vertical axis), (a). Visible higher harmonics in the GMR spectrum come from the appearance of higher harmonics in the oscillations in Co layer. The temporal evolution of the average magnetization $\mathrm{x}$-component below the PC and the associated GMR that served for the calculation of the spectra, (b). The same color legend for both subfigures.

presented in Fig. 5.24. Co vortex trajectory is represented as a superposition of two different movements, first one being ccw and yielding the base frequency. Thus, magnetostatic interaction with the ccw moving core in $\mathrm{Py}$ layer (positive polarity) drives Co vortex, against right hand rule, in the ccw direction. Second, the cw eigenoscillations yield additional higher harmonics. While three (3f) and seven times (7f) the base frequency are observed in spectrum representing the Co layer, the fourth and eighth harmonics are visible in the GMR spectrum. Hence, the $3 \mathrm{f}$ and $7 \mathrm{f} \mathrm{cw}$ movements of Co vortex combine with the ccw $\mathrm{f}$ movement of vortex in Py layer and results in $3 \mathrm{f}+\mathrm{f}=4 \mathrm{f}$ and $7 \mathrm{f}+\mathrm{f}=8 \mathrm{f}$ in the corresponding GMR spectrum. Recall that for a vortex pair gyrating in common direction the output GMR represents the difference of the eigenfrequencies (Fig. 5.8). 
The vortex in Py layer gyrates ccw on a well defined orbit that is independent of current (compare $-8 \mathrm{~mA}$ and $-12 \mathrm{~mA}$, Fig. 5.23b). The amplitude of the GMR signal decreases with the absolute current value as a result the current dependent displacement of the vortex core in Co layer. This complex superposition of frequencies and the existence of well defined oscillations in Py layer, where no ST is present, indicate the significance of the magnetostatic contribution to the synchronization of the vortices, i.e. in absence of magnetostatic coupling to the ccw moving vortex in Py, the vortex in Co layer would undergo simple $\mathrm{cw}$ movements owing its negative polarity. Moreover, the sum of frequencies appears only as a side peak in the read out GMR spectra because Co vortex remains inside the PC. Both vortices moving in opposite directions outside the PC would yield the frequency sum as the main output.

At the absolute current of $16 \mathrm{~mA}$ the $7 \mathrm{f}$ mode in Co layer is suppressed which results in change of vortex orbit in Py and the appearance of an upward jump in the associated GMR amplitude, Fig. 5.25. On the contrary to previously reported feature: Py vortex orbit remained current independent within ranges $(0 \mathrm{~mA},-6 \mathrm{~mA})$ and $(-7 \mathrm{~mA},-15 \mathrm{~mA})$ with a single jump defining two fixed orbits, above $16 \mathrm{~mA}$ Py vortex orbit shrinks. This can be understood as follows. At larger currents the effective destabilizing effect induced by ST on Co layer becomes dominant over the magnetostatic interactions. Recall Fig. 5.17f where the transmitted electrons are polarized with the same chirality as the vortex state in the target Co layer. Thus, the destabilizing effect, once Py vortex approaches the PC area, comes solely from the combination of the positive OP momentum carried by the polarized electrons impinging at the negative polarity target vortex. This favors core switching but not the lateral displacement of the vortex in the target. However, before the core can be switched vortex must reach certain critical velocity. Hence, at high currents the increase of vortex velocity translates into steeper slope of the time derivative of the overall magnetization state in 
Co layer. This effect is the only driving force for the vortex in Py layer as it provides the energy to sustain the oscillations. Thus, the current dependent increase of Co vortex velocity introduces a current dependence into the Py vortex displacement.

At the critical (absolute) current of $51 \mathrm{~mA}$ the vortex in Co layer changes the polarity. The configuration of two vortices of common polarity and chirality is then stabilized and no dynamics is observed above this current.

Inversing the direction of current leads to behavior discussed in Section 5.4.1.

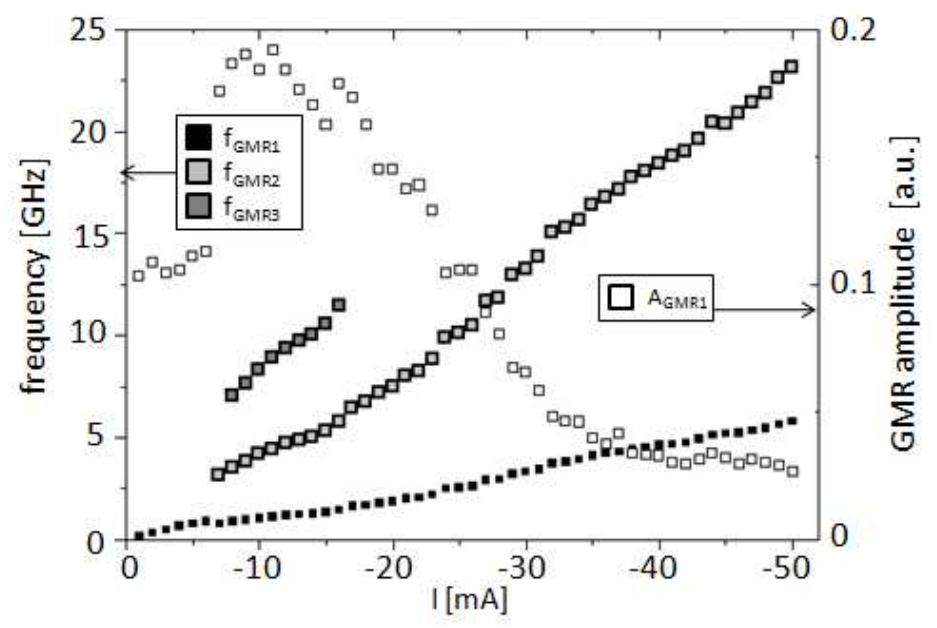

Fig. 5.25: ST active on Co layer. Output frequency evolution with current, together with the higher harmonics and the resulting amplitude of the GMR signal.

ST active on Py and Co layers

Activating ST effect on both layers leads to following observations. At the threshold current Py vortex is expelled from the the PC and it oscillates on 


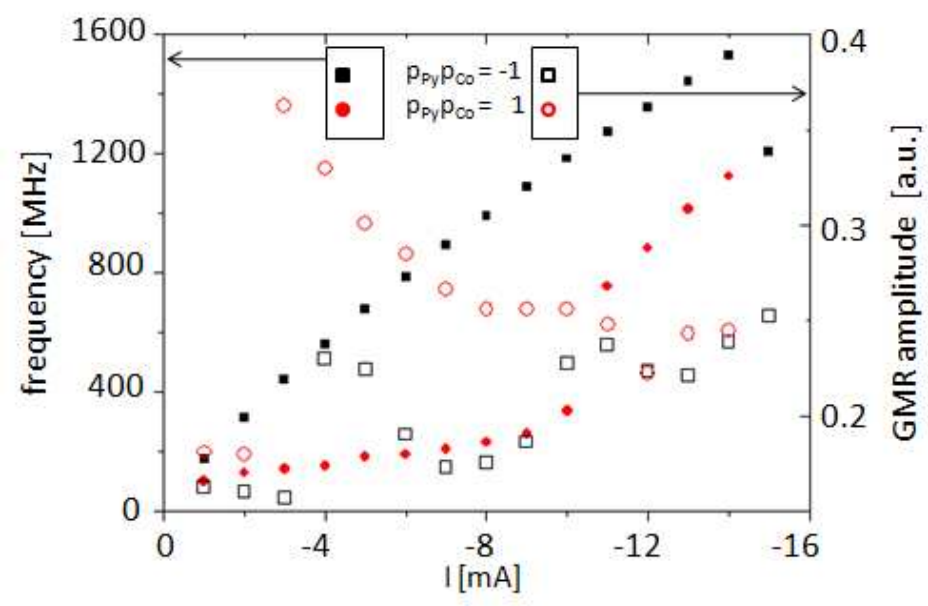

Fig. 5.26: Left axis: frequency as function of current when both $\mathrm{Py}$ and Co are subject to ST effect, both vortices of the same - red full circles and opposite - black full squares polarity, only the base frequency is shown. Right axis: the associated GMR amplitude with open symbols.

the same orbit as presented in Fig. 5.19b with the ccw direction of gyration. The resulting frequency is in the same order of magnitude but the associated GMR amplitude is similar to the case of the same vortex polarity only in the low current range, Fig. 5.26. The differences in the behavior become visible as the absolute current is increased. Frequency blueshift with (absolute) current is much faster for the case of opposite vortex polarities.

Below the absolute current of $4 \mathrm{~mA}$ one single peak (full black squares, Fig. 5.27b) in the frequency spectra is observed corresponding to the eigenfrequency of coupled vortices whose oscillation amplitude stays constant (full black circles, Fig. 5.27a). The current of $-4 \mathrm{~mA}$ seems to be the threshold for the ST-driven dynamics in Co layer because the vortex within starts oscillating inside the PC area. No expansion of the vortex displacement is observed. Still, as explained earlier, ST dependent velocity of vortex in Co 
layer increases steepening the potential well sustaining vortex oscillations in Py layer thus influencing the displacement of the core in this layer.

A complicated superposition of two frequencies in Co layer is observed: ccw movement results in low frequency movement (f), while cw movement results in the appearance of $3 \mathrm{f}$ mode. Taking into account that the direction of the high frequency $(\mathrm{cw})$ movement of the vortex in Co layer is opposite to the direction of vortex gyration in Py layer (ccw) the $3 \mathrm{f}$ peak appearing in Co spectrum translates into the sum of both frequencies: $\mathrm{f}+3 \mathrm{f}=4 \mathrm{f}$ in the GMR spectrum $\left(\mathrm{GMR}_{2}\right)$, see red circles in Fig. 5.27b. The appearance of this second frequency results in the decrease of the peak amplitude of the base mode and the upward jump of the associated GMR signal followed by its decrease as the absolute current is increased, see Fig. 5.27a.

At $-7 \mathrm{~mA}$ the spectrum extracted from the data corresponding to $\mathrm{PC}$ in Co layer becomes even more complicated and additional peak at $2 \mathrm{f}$ is found. This peak leads, as discussed previously, to the appearance of the $3 \mathrm{f}$ peak in the GMR spectrum $\left(\mathrm{GMR}_{3}\right.$, full green squares Fig. 5.27b). The appearance of second harmonic in Co spectrum is followed by the increase of the GMR amplitude down to the current of $-10 \mathrm{~mA}$. At this current an additional side peak is observed in the GMR spectra $\left(\mathrm{GMR}_{4}\right)$, marked by full blue squares in Fig. 5.27b, corresponding to the $2 \mathrm{f}$ mode i.e. high frequency cw Co vortex core movement is superposed with the low frequency Py vortex core ccw movement resulting in the frequency $3 \mathrm{f}-\mathrm{f}=2 \mathrm{f}$. After the appearance of this mode the GMR amplitude stays constant down to the critical current of $-15 \mathrm{~mA}$ where the Py vortex velocity exceeds the critical value and changes the polarity. Thus, the reappearance of the mode as reported for a system of two vortices of common chirality and polarity is observed, Fig. 5.26. 

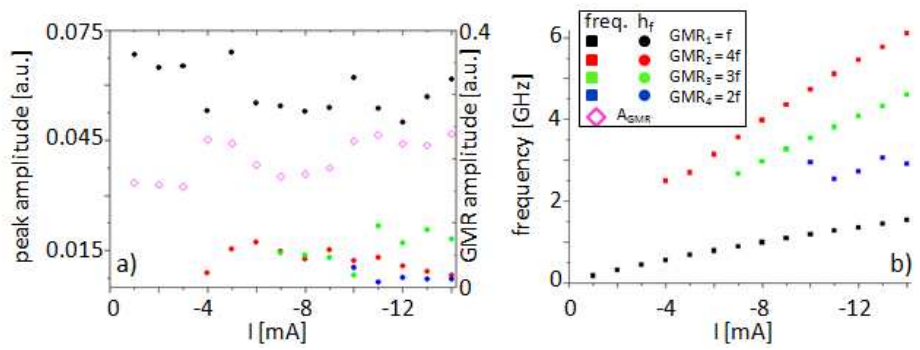

Fig. 5.27: Left axis: frequency as function of current when both Py and Co are subject to ST effect, both vortices of the same - red full circles and opposite - black full squares polarity. Right axis: the associated GMR amplitude with open symbols.

\subsection{Two equal ferromagnets}

Note that considering two vortices of opposite polarity leads (II in Fig. 5.17) to the decrease of the output GMR amplitude as compared to the case of common polarity ( $\mathrm{I}$ in Fig. 5.17) if only the low $\mathrm{M}_{\mathrm{s}}$ ferromagnetic layer is subject to ST, Figs. 5.19a. This quantitative change in terms of the GMR amplitude is rather small compared to the increase of the quantitative frequency response. Thus, manipulating the relative polarity of vortices in a sample where the low $\mathrm{M}_{\mathrm{s}}$ ferromagnetic layer is $\mathrm{ST}$ active tunes frequency an order of magnitude leaving almost unchanged the output amplitude of the signal.

When activating ST solely on the high $\mathrm{M}_{\mathrm{s}}$ ferromagnet (Co) the change of relative polarity brings about the need of changing current direction in order to sustain the output, Fig. 5.22. Larger output signal amplitude is observed in I as compared to II but very similar quantitative result is reported in terms of frequency (in the current range where both vortices preserve their initial polarities). Thus, the choice of polarities rather than influencing the 
frequency defines the output signal amplitude (quantitatively).

The above observations can be easily understood recalling that low $M_{s}$ ferromagnet is more susceptible to ST and that the relative displacement between the cores depends via ST effect on the polarities of the cores.

Hence, if both layers are subject to ST, Fig. 5.26, quite significant increase in terms of frequency and decrease in terms of signal amplitude (comparing II to I) are reported but without the need of changing current direction to preserve the dynamics. Thus, the qualitative behavior is largely defined by the ST acting on a susceptible low $\mathrm{M}_{\mathrm{s}}$ ferromagnet and the magnetostatic interactions.

In the following, symmetric spin valves are investigated in order to determine the importance of the material on the system response in terms of frequency and amplitude of output signal. Term symmetric corresponds to trilayers where both ferromagnetic layers are made of the same material, $\mathrm{Py}(5 \mathrm{~nm}) / \mathrm{Cu}(5 \mathrm{~nm}) / \mathrm{Py}(5 \mathrm{~nm})$ and $\mathrm{Co}(5 \mathrm{~nm}) / \mathrm{Cu}(5 \mathrm{~nm}) / \mathrm{Co}(5 \mathrm{~nm})$.

\subsubsection{Common polarity}

Recall cases yielding dynamic output assuming vortices of common chirality and polarity as presented in Fig. 5.17. Here, the study is carried out for the trilayer, where the ferromagnetic layers are made of the same material and compared to previously obtained results. Figure 5.28 summarizes the main findings which are to be discussed in detail in the following. Note that the electrons are assumed to flow from top to bottom layer defining the chirality of both vortices.

Sustained oscillations are reported in cases denoted as $\left(\mathrm{a}_{1}\right)$ and $\left(\mathrm{c}_{1}\right)$ which analogous to (a) and (c) represent ST activated only on the top layer. Thus, in $\left(\mathrm{a}_{1}\right)$ and $\left(\mathrm{c}_{1}\right)$ the polarizer is made of the same material as the target layer (Py and Co corresponding to trilayers $\mathrm{Py} / \mathrm{Cu} / \mathrm{Py}$ and $\mathrm{Co} / \mathrm{Cu} / \mathrm{Py}$, respectively). 


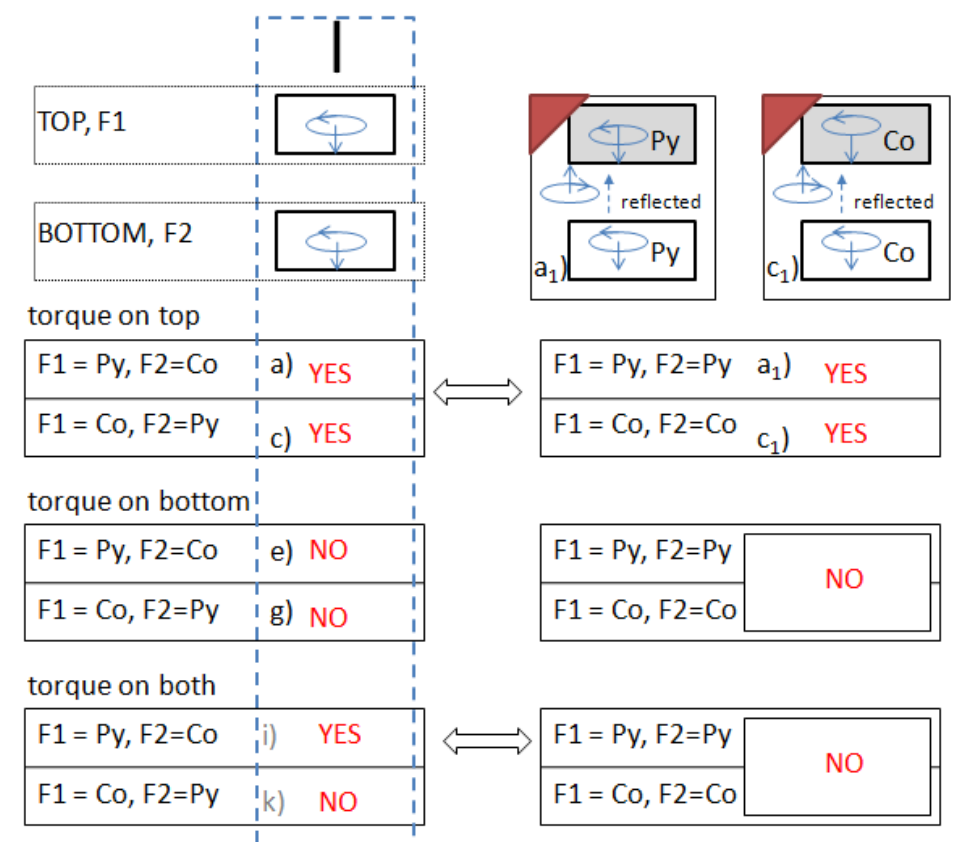

Fig. 5.28: Vortices of the same chirality and polarity are considered. The results obtained for the asymmetric trilayer are summarized in the left panel (as $\mathrm{I}$ in Fig. 5.17). In case of symmetric valves $\mathrm{Py} / \mathrm{Cu} / \mathrm{Py}$ and $\mathrm{Co} / \mathrm{Cu} / \mathrm{Co}$ dynamics is observed in $\left(\mathrm{a}_{1}\right)$ and $\left(\mathrm{c}_{1}\right)$, whose results are to be compared to (a) and (c), respectively.

$$
\operatorname{Py}(5 \mathrm{~nm}) / \mathrm{Cu}(5 \mathrm{~nm}) / \operatorname{Py}(5 \mathrm{~nm})
$$

Comparing $\left(a_{1}\right)$ to $(a)$ one concludes that the qualitative behavior remains similar, thus, quantitatively some differences are observed, Fig. 5.29. Following qualitative similarities are observed:

- frequency blueshift with current

- vortex orbit expansion (in top layer) with current, Fig. $5.29 \mathrm{c}$

- vortex oscillations within PC in the bottom layer

- existence of the cut-off current and core polarity switching above 
From the quantitative point of view, except for the similar GMR amplitude, the response is rather different. Comparing $\left(\mathrm{a}_{1}\right)$ to $(\mathrm{a})$ :

- larger vortex displacement in top layer, Fig. 5.29b

- lower cut-off current

- lower frequency values

- almost constant frequency throughout the whole current range in $\left(\mathrm{a}_{1}\right)$ are observed. The first can be understood as follows. Recall that the only driving force for the oscillations to be observed in the bottom layer where no ST in present is the coupling field with the vortex moving in the top layer. Thus, part of the energy that is pumped via ST effect into top layer is converted into the vortex gyration within and small part is coupled back to the bottom layer where minor oscillations are observed. Even though core-core magnetostatic interaction is stronger, the energy cost of sustaining oscillations in the bottom layer is larger, in (a) where high $\mathrm{M}_{\mathrm{s}}$ ferromagnetic material is considered than in the case of low $\mathrm{M}_{\mathrm{s}}$ ferromagnet, $\left(\mathrm{a}_{1}\right)$. Thus, in the latter more energy is directly translated into sustaining oscillations in the top layer and, therefore, vortex displacement is larger and cut-off current is lower.

The reason for reporting lower frequency values as comparing $\left(\mathrm{a}_{1}\right)$ to $(\mathrm{a})$ is trivial. The change of the material the polarizer is made of changes the magnetostatic coupling field, which is weaker in case of lower $\mathrm{M}_{\mathrm{s}} \mathrm{Py}$ polarizer, $\left(\mathrm{a}_{1}\right)$, and, thus, the overall energy balance in the top layer is changed leading to lower output frequency.

Explaining the last observation from the list pointed out above is more complex. In general, frequency of vortex oscillations shows to be strongly dependent on current. In $\left(a_{1}\right)$, however, the frequency stays almost constant over the whole current range, where well defined oscillations are observed. Nevertheless, in (a) the observed frequency blueshift with current is also 
rather slow. It can be explained as follows. Once the cores are very far apart any further change of the cores distance is not that crucial in terms of changing internal fields within the structure, which are responsible for frequency determination. Thus, considering that the vortex core displacement in the top layer in $\left(\mathrm{a}_{1}\right)$, Fig. 5.29b, is very large the (almost) current independent frequency is to be expected.

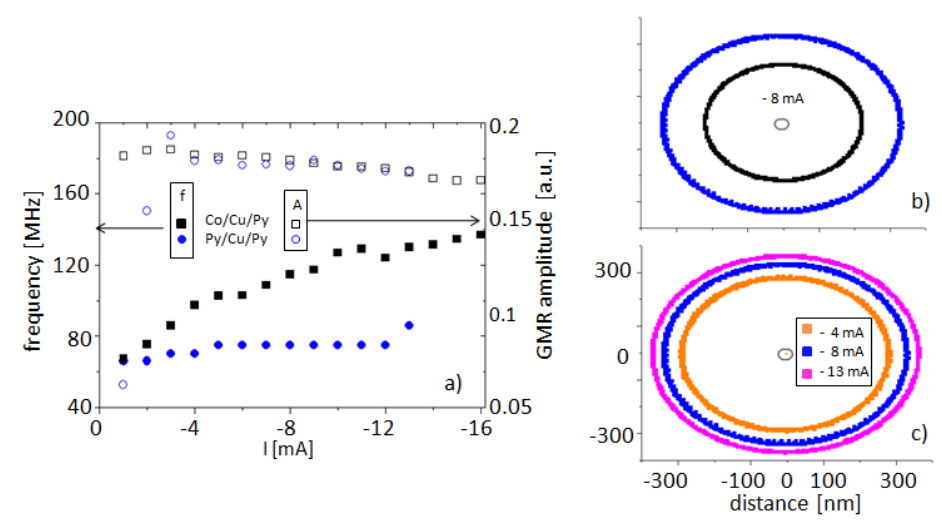

Fig. 5.29: Top Py layer is the active layer, while $\mathrm{Py}$ or Co are considered for the polarizer: $\mathrm{Co} / \mathrm{Cu} / \mathrm{Py}$ and $\mathrm{Py} / \mathrm{Cu} / \mathrm{Py}$, respectively. Thus, in the color legend black corresponds to results obtained in the first, while blue in the latter. Left axis, full symbols: frequency as function of current; right axis, open symbols: the corresponding amplitude of the GMR signal, (a). Comparison of the vortex orbit in the top Py layer at the current of $-8 \mathrm{~mA}$, (b). $\mathrm{Py} / \mathrm{Cu} / \mathrm{Py}$, vortex orbit expansion with current, (c).

Note, that close to the threshold current the amplitude of the GMR amplitude increases rapidly when assuming the polarizing layer to be made of $\mathrm{Py}(\mathrm{Py} / \mathrm{Cu} / \mathrm{Py})$, while it remains almost constant when Co polarizer is considered $(\mathrm{Co} / \mathrm{Cu} / \mathrm{Py})$, open symbols, Fig. 5.29a. This difference in the GMR amplitude at the between the currents of $-1 \mathrm{~mA}$ and $-2 \mathrm{~mA}$ arises from 
the rearrangement of the relative displacement of the vortices. Thus, at the threshold of $-1 \mathrm{~mA}$ both are expelled from the PC but surprisingly vortex in the bottom Py layer, even though no ST effect is activated there, is expelled further than the corresponding vortex in the top layer, that is under the influence of ST, Fig. 5.30b. Hence, having plotted the average $\mathrm{x}$-component of the magnetization inside the PC in both layers one concludes that vortices oscillate out-of-phase, Fig. 5.30a, which leads to the destruction of the output signal amplitude. Already at $-2 \mathrm{~mA}$ the ST drives the vortex in the top layer further away from the PC. At the same time the other vortex decays to oscillate inside the PC, Fig. 5.30c and d, explaining the observed initial jump in the GMR amplitude.

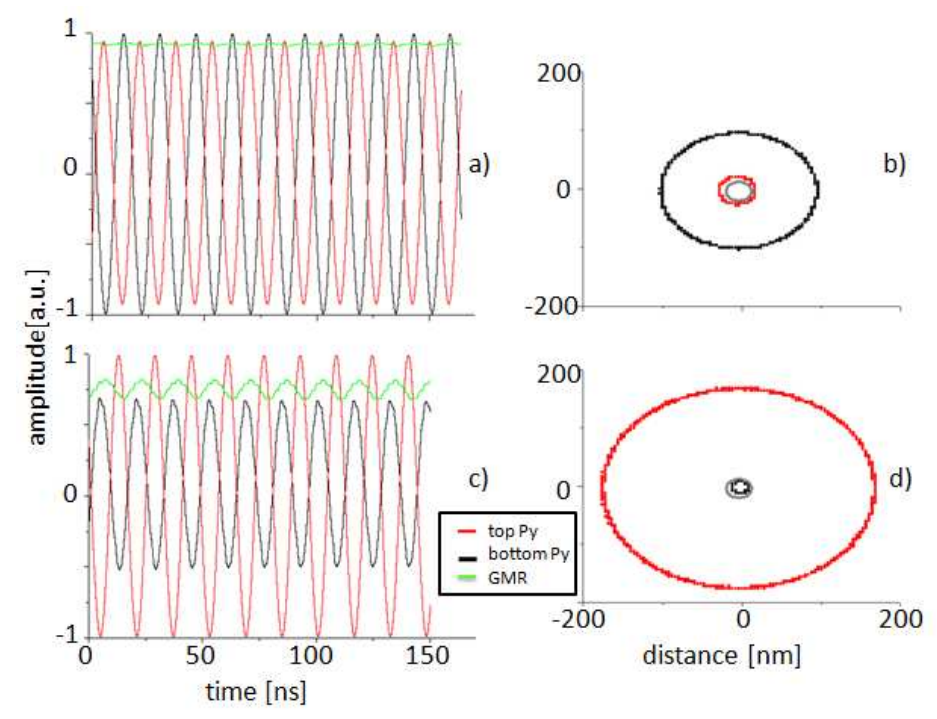

Fig. 5.30: $\mathrm{Py} / \mathrm{Cu} / \mathrm{Py}$, the average magnetization $\mathrm{x}$-component extracted from the configuration in the PC in bottom (black) and top (red) layers together with the resulting GMR amplitude (green) at $-1 \mathrm{~mA}$ and $-2 \mathrm{~mA}$, (a) and (c), respectively. The corresponding orbits of vortices moving in the top and bottom layers, (b) and (d). 
At the cut-off current of $-14 \mathrm{~mA}$ the vortex in the top layer preserves its negative polarity and continues its cw gyration very far from the PC. In the middle of the sample the magnetization OP component undergoes periodic switching which is followed by the spin wave radiation. On the other hand, vortex in the bottom layer remains very close to the PC exhibiting systematic core polarity switching which is accompanied by the change of the gyration direction. This additionally triggers spin waves generation that expand outwards. These highly complex processes in both layers give rise to irregular output signal and the decrease of its amplitude.

The situation becomes even more complicated at $-15 \mathrm{~mA}$. In the bottom layer, where no ST is present, magnetostatically triggered periodic core polarity switching is observed, which is followed by the spin wave generation. In the top layer, however, the temporal evolution is more complex. At first the vortex gyrates in a ccw direction (owing its negative polarity) far from the $\mathrm{PC}$ which is why it is not influenced by the spin waves nucleated in the PC. Thus, the local distortion of the magnetic configuration owing the spin wave propagation soon affects the vortex evolution. First a V-AV pair (both of negative polarity) is created inside the PC. Numerous complicated dynamic processes are observed: antivortex switches the polarity and the pair is being expelled from the PC. Soon the vortex separates from the antivortex and is destroyed by the spin wave radiation while the antivortex continues moving on a complex trajectory. Thus, the initial vortex changes the polarity and when it gets closer to the antivortex an additional $\mathrm{V}-\mathrm{AV}$ pair of positive polarity is created. This $\mathrm{V}-\mathrm{AV}$ quadrupole is soon annihilated and a single negative polarity vortex is created in the PC. It it expelled from the PC and starts to gyrate close to the $\mathrm{PC}$ area accompanied by the spin wave radiation. Subsequent core polarity switching is observed followed by the change of the gyration direction. Finally, the vortex decays to the PC and remains there undergoing complicated dynamics including spin wave nucleation and creation and expulsion of the antivortices. These processes are highly inter- 
esting but lead to chaotic readout signal and are far beyond the interest of this work.

$$
\mathrm{Co}(5 \mathrm{~nm}) / \mathrm{Cu}(5 \mathrm{~nm}) / \mathrm{Co}(5 \mathrm{~nm})
$$

Consider now a trilayer, where both ferromagnetic layers are made of Co. At the test current the dynamics is observed only if the top layer is subject to ST. Therefore, a comparison between $(c)$ and $\left(c_{1}\right)$ of Fig. 5.28 can be carried out systematically. In general, similar frequencies are observed, Fig. 5.31, and the vortex orbit in top Co layer in both cases shrinks as the current is increased.

On the other hand, numerous differences can be pointed out. First of all the mobility of vortex in the bottom layer is larger in (c) than in $\left(c_{1}\right)$. Hence, the ST effect is active only on the top layer and only part of the energy is transferred back magnetostatically to the bottom layer. This one in (c) is made of $\mathrm{Py}$ and in $\left(c_{1}\right)$ of Co which justifies the observation of larger displacement of vortex in the first. Thus, in $\left(c_{1}\right)$ the vortex in the bottom layer remains inside the $\mathrm{PC}$ throughout the studied current range. This, in turn, leads to lower output amplitude of the GMR signal and the existence of one sole frequency in the reported spectra. Both oscillators yield the same frequency which corresponds to the final eigenfrequency reported in the system. In (c) each layer supported different frequency mode and the GMR readout was the difference between the eigenfrequencies of each oscillator.

Moreover, the GMR amplitude in both cases decreases with current but the nonlinear and discontinuous behavior in (c) is rather more complex as compared to the monotonic tendency observed in $\left(c_{1}\right)$. On the other hand, the corresponding frequency exhibits nonlinear blueshift with current. Purely ST-driven motion of magnetic vortex shall, in principle, be characterized by a linear frequency increase with current owing the proportionality between the ST and the applied current value. Thus, the above mentioned nonlinear be- 
havior must arise from the magnetostatic interactions with the bottom layer. These interactions are stronger in case of using ferromagnetic material characterized by high $M_{s}$. Therefore, in (c) where Py is assumed for the material of the polarizer the magnetostatic contribution does not affect the linearity of the frequency blueshift with current. On the contrary, in $\left(c_{1}\right)$, where the polarizer is made of higher $\mathrm{M}_{\mathrm{s}}$ Co the presence of the magnetostatic contribution is manifested by the appearance of the nonlinear frequency blueshift with current.

This trend is observed up to the critical current of $20 \mathrm{~mA}$, above which vortex in the top layer decays to oscillate close to the $\mathrm{PC}$ reaching the critical velocity for core reversal. As a result change of the gyration direction and the generation of spin waves are observed. Thus, this mode is not stable and successive switching of polarity is observed. Notably, each such event is followed by the vortex expulsion from the PC. Thus, the velocity of oscillating vortex increases again and the subsequent polarity flip is reported. On the other hand, vortex in the bottom layer remains inside the PC preserving its polarity and oscillating with minor amplitude. However, the complicated processes in the top layer lead to the irregular and low output signal. Therefore, the study is not continued for larger currents.

\section{Static cases}

In case of activating the ST on bottom layer no oscillations are observed, because independent of the layer material the resulting ST is acting stabilizing on the configuration in the bottom layer. While some remaining net torque could be expected in case of two different ferromagnets due to core size differences in each material resulting in some initial dynamic effect, no such behavior is foreseen when assuming equal ferromagnets. Thus, no dynamics is observed, analogous to (e) and (g) from Fig. 5.17. 


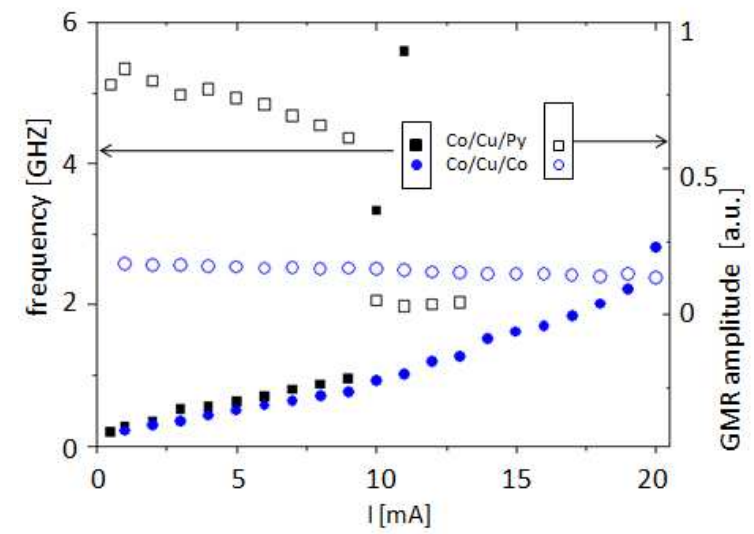

(a)

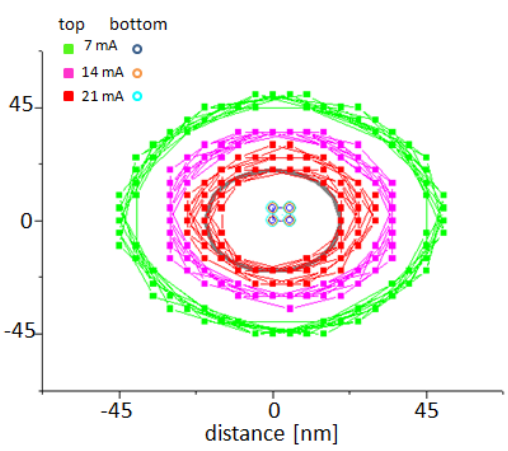

(b)

Fig. 5.31: Top Co layer is the active layer, while $\mathrm{Py}$ or Co are considered for the polarizer: $\mathrm{Co} / \mathrm{Cu} / \mathrm{Py}$ and $\mathrm{Co} / \mathrm{Cu} / \mathrm{Co}$, respectively. Thus, in the color legend black corresponds to results obtained in the first, while blue to the latter. Left axis, full symbols: frequency as function of current; right axis, open symbols: the corresponding amplitude of the GMR signal, (a). The orbit dependence on current of vortices in top and bottom layers, (b).

Similarly, when activating the ST on both layers simultaneously, owing the identity of the initial configuration in both, no initial ST is present in the system and as a consequence no dynamics output is reported.

\subsubsection{Opposite polarity}

Recall the dynamic cases when assuming vortices of opposite polarity in an asymmetric trilayer (II in Fig. 5.17). Considering symmetric structure the output analogous to (b), (f) and ( $\mathrm{j}$ ) is reported but only when Py is chosen the ferromagnetic material. In $\mathrm{Co} / \mathrm{Cu} / \mathrm{Co}$ trilayer the initial state of vortices of common chirality but opposite polarity is not stable (at the test current). Thus, in the dynamic study one of the vortices changes the polarity which leads to the results as described above where common polarity case 
was considered. This leads to the conclusion that in a trilayer where high $\mathrm{M}_{\mathrm{s}}$ Co ferromagnet is employed the minimization of magnetostatic energy favoring the common polarity dominates the ST effect (at the test current) and, therefore, core switching event is observed already at low current. On the other hand, using ST susceptible $\mathrm{Py}$ in a $\mathrm{Py} / \mathrm{Cu} / \mathrm{Py}$ yields dynamic output as presented schematically in Fig. 5.32. The results of systematic study are discussed in detail for each case in the following paragraphs.

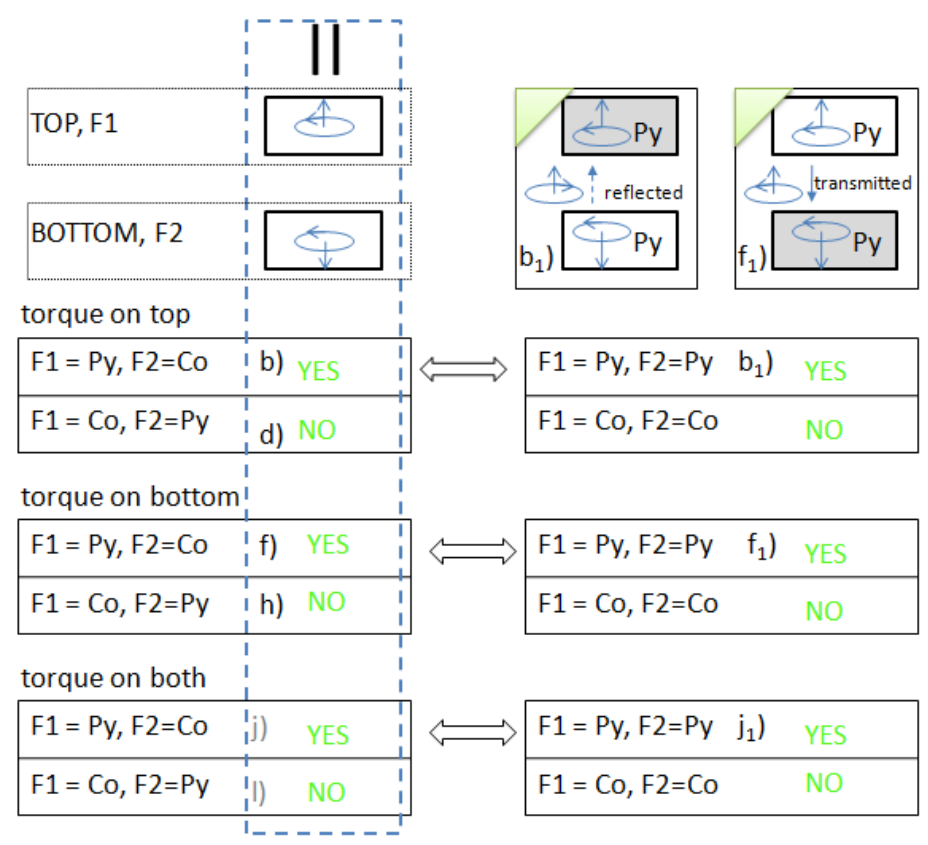

Fig. 5.32: Vortices of the same chirality and opposite polarity are considered. The results obtained for the asymmetric trilayer are summarized in the left panel (as II in Fig. 5.17). Assuming symmetric valves the dynamics is reported only if $\mathrm{Py}$ is chosen as the material for the ferromagnetic parts of the trilayer. Sustained oscillations arising from $\left(b_{1}\right)$ and $\left(f_{1}\right),\left(j_{1}\right)$ are systematically studied and compared to (b), (f) and (j), respectively. 


\section{ST on top layer}

Consider first the ST effect active on the top layer. If both layers are made of Py, $\left(b_{1}\right)$, dynamics response analogous to (b) is observed, Fig. 5.32. Similar frequencies and GMR amplitudes are reported throughout the studied current range, Fig. 5.33a. Thus, slightly larger frequency as comparing $\left(\mathrm{b}_{1}\right)$ to (b) is further associated with a more confined orbit, Fig. 5.33b. Notably, along with favoring positive polarity in the top Py layer (OP ST), the overall ST effect increases the vortex velocity, which finally leads to the switch of vortex polarity below the critical current of $-8 \mathrm{~mA}$, so that trends presented before in Fig. 5.29 (vortices of common polarity) are continued.

The reason for observing slightly higher frequency values as comparing $\left(b_{1}\right)$ to $(b)$ is analogous to the discussion carried out when comparing $\left(a_{1}\right)$ to (a). Just that now the OP component of the ST and the core-core interaction change the sign. Thus, considering Py polarizer, $\left(b_{1}\right)$ the overall energy balance in the top layer is changed leading to slightly lower vortex displacement and higher output frequency, Fig. 5.33. Still, qualitatively current independent orbit is reported. The explanation of this observation has been delivered in previous paragraphs.

\section{ST on bottom layer}

Recall that as shown in Fig. 5.32 (initial vortices of opposite polarity) if only the bottom layer was subject to ST the dynamic response was reported assuming polarizer made of Py (top) and the ST active Co layer (bottom), which corresponded to subfigure (f). In the following both layers are assumed to be made of Py and the studied case is denoted as $\left(\mathrm{f}_{1}\right)$. Thus, there are quite a few differences in the dynamic response as comparing results arising from $\left(\mathrm{f}_{1}\right)$ and $(\mathrm{f})$. 


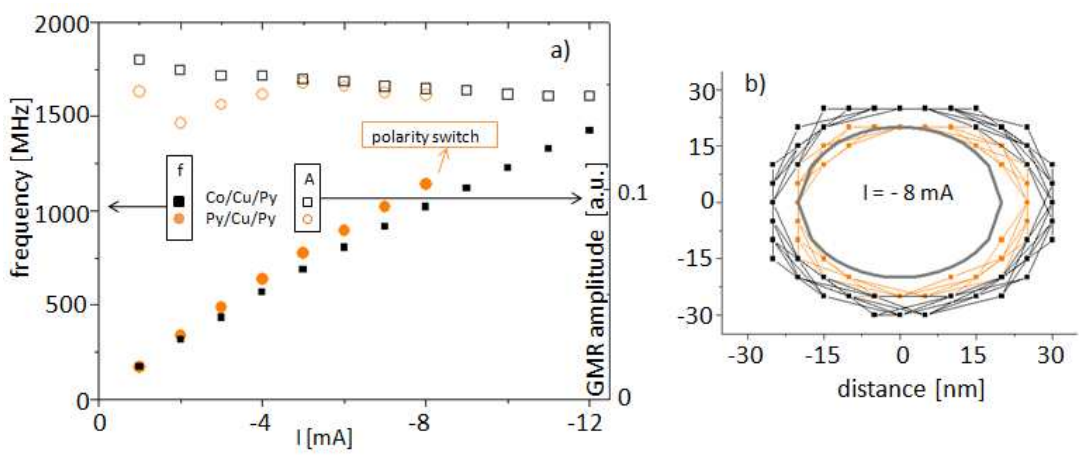

Fig. 5.33: ST active on the top Py layer. Frequency and GMR amplitude dependence on current, compared for the trilayers $\mathrm{Cu} / \mathrm{Co} / \mathrm{Py}$ in black and $\mathrm{Py} / \mathrm{Co} / \mathrm{Py}$ in orange, (a). Comparison of the vortex orbit in the top Py layer at the current of $-8 \mathrm{~mA}$, (b). The same color legend for both subfigures.

First the comparison in terms of frequency and the output signal amplitude shall be carried out, Fig. 5.34. Following quantitative differences are observed as compared $\left(\mathrm{f}_{1}\right)$ to $(\mathrm{f})$ :

- slightly lower frequency

- four times larger threshold for the appearance of higher harmonics

- lower critical current (twice)

Hence, in both cases frequency blueshift with current is observed. As has been explained earlier the nonlinear character of the frequency increase with current in $\left(\mathrm{f}_{1}\right)$ is the consequence of the fact that the ST driven dynamics is additionally assisted by the magnetostatic interactions between the cores. Moreover, a lower critical current is reported because critical velocity for the core reversal in the symmetric valve where Py layer is subject to ST is reached at lower currents as compared to ST active Co layer in (f). As a result of the polarity switch of the vortex in the bottom layer the overall configuration in represented by two vortices of equal chirality and polarity. The transferred 
electrons polarize in the top layer exactly parallel to the state in the target bottom layer so that no effective ST is present at the interface. Therefore, the oscillations are damped. Note, that qualitatively the same behavior at critical current is observed in $\left(\mathrm{f}_{1}\right)$ and $(\mathrm{f})$.

The reason for reporting higher frequency values is straightforward. Recall Fig. 5.33a. The frequency in the symmetric $\mathrm{Py} / \mathrm{Cu} / \mathrm{Py}$ trilayer was slightly higher than in case of the asymmetric structure when assuming ST active on the top layer. The explanation was based on the statement that in case of Py polarizer, $\left(b_{1}\right)$ Fig. 5.32 the overall energy balance in the top layer was changed because of the use of low $\mathrm{M}_{\mathrm{s}}$ ferromagnetic material, Fig. 5.33. Here, however the frequency as compared $\left(f_{1}\right)$ to $(f)$ is slightly higher. This can be justified as follows. In $\left(b_{1}\right)$ Fig. 5.32 the ST acting on the top layer arises from the reflected electrons. Thus, the IP ST destabilizes the vortex in the top layer from the aligned position but the OP ST stabilizes the core in the central position. On the other hand in $\left(f_{1}\right)$ the ST exerted on the bottom layer arises from the transmitted electrons. Hence, only the OP ST is acting destabilizing while the IP ST favors aligned position. Thus, the corresponding displacement of the vortices as compared $\left(f_{1}\right)$ to $(f)$ is smaller and the frequency higher. This presence of the OP ST is also the cause of lower critical current for core reversal observed in $\left(\mathrm{f}_{1}\right)$.

Having considered above explanations the reason why the threshold for the appearance of higher harmonics at higher currents in $\left(f_{1}\right)$ becomes clear. In presence of ST on one layer, the dynamics in the adjacent layer and in what follows the appearance of higher harmonics arise from the magnetostatic interactions between the ST driven and free vortex. There interactions depend on the material parameters. Thus, the core-core interaction in a vortex pair of opposite polarity in $\mathrm{Co} / \mathrm{Cu} / \mathrm{Py}$ trilayer is stronger than in $\mathrm{Py} / \mathrm{Cu} / \mathrm{Py}$ case which is why the first support higher harmonics at lower currents. 


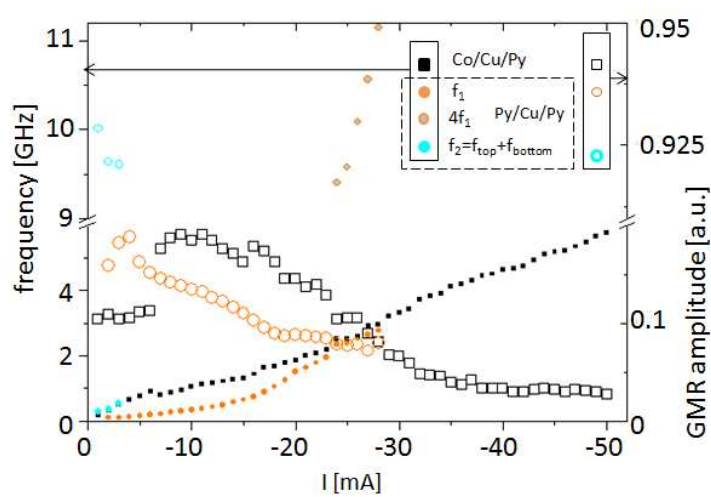

(a)

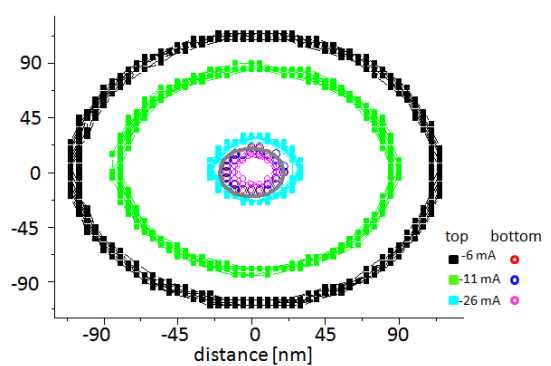

(b)

Fig. 5.34: ST active on the bottom Py layer. Frequency and GMR amplitude dependence on current, compared for the trilayers $\mathrm{Cu} / \mathrm{Co} / \mathrm{Py}$ in black and $\mathrm{Py} / \mathrm{Co} / \mathrm{Py}$ in orange, (a). The orbit of the vortices in both layers at different currents, (b).

Consider the oscillations close to the threshold current, Fig. 5.35a. At the current of $-1 \mathrm{~mA}$ only one frequency is present in the GMR spectrum and it corresponds to the sum of frequencies supported in each ferromagnetic layer, Fig. 5.35b. This is the result of both vortices oscillating in opposite directions outside the PC each with their own eigenfrequencies, Fig. 5.35c, which leads to large output amplitude. Thus, down to $-3 \mathrm{~mA}$ the orbit of the vortex in the top layer expands with current, while in the bottom layer vortex remains close to the PC edge (not shown). However, already at $-2 \mathrm{~mA}$ two distinct modes are observed, Fig. 5.36. In addition to previously present high frequency $\mathrm{GMR}_{2}$ a distinct low frequency oscillations are observed in certain time windows, $\mathrm{GMR}_{1}$. The appearance of this frequency is the result of the magnetostatic coupling between the cores, which forces common gyration direction of both vortices. Moreover, the vortex in bottom layer decays to PC which below $-3 \mathrm{~mA}$ is followed by the disappearance of the high frequency 


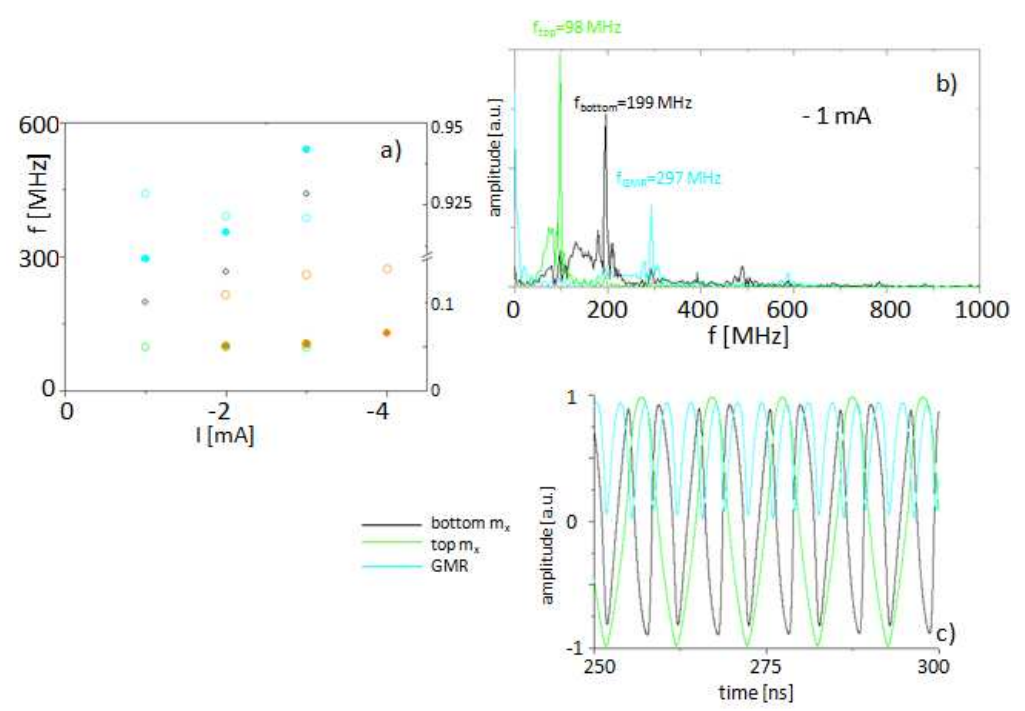

Fig. 5.35: Frequency and the GMR amplitude as in Fig. 5.37 but only at low currents, (a). Frequency spectra obtained at $-1 \mathrm{~mA}$ for the data readout inside the PC in top (green), bottom (black) layers; the resulting GMR (light blue) corresponds to the sum of these frequencies, (b). Temporal evolution of average magnetization $\mathrm{x}$-component inside the $\mathrm{PC}$ in top and bottom layers and the associated GMR, (c).

mode. The associated orbit of vortex in the top layer starts decreasing with current, Fig. 5.33b. This phenomenon has been previously elaborated on.

The appearance of the high frequency mode, its coexistence with the low frequency mode and its disappearance at currents very close to threshold is a very interesting and complex effect.

\section{ST on both layers}

Consider now the case of both layers set subject to the ST and compare dynamic output reported in the symmetric $\mathrm{Py} / \mathrm{Cu} / \mathrm{Py},\left(\mathrm{j}_{1}\right)$, to the asymmetric 


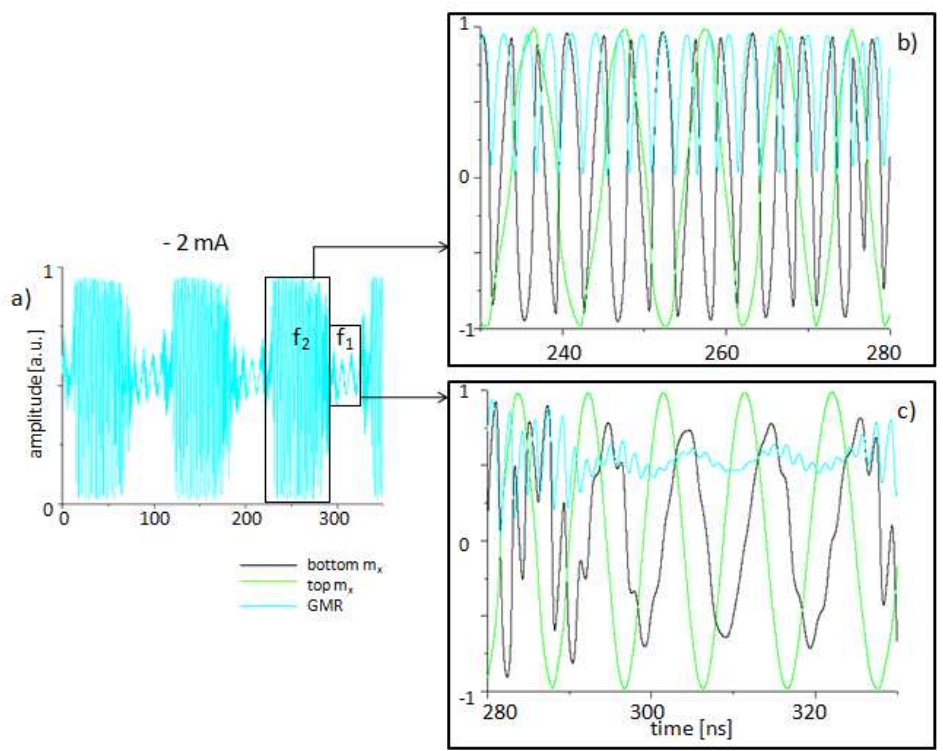

Fig. 5.36: Temporal evolution of the GMR signal, (a) together with the evolution of the average magnetization $\mathrm{x}$-component inside the $\mathrm{PC}$ in top and bottom layers in different time windows corresponding to high and low frequency mode, (b) and (c), respectively.

valve $\mathrm{Cu} / \mathrm{Co} / \mathrm{Py},(\mathrm{j})$, Fig. 5.32. Note that different stabilizing and destabilizing effects are present in both layers. The polarity of the vortex in the top layer is stabilized via OP ST from the reflected electrons, while the central position of the vortex is destabilized by the IP ST. In the bottom layer, in turn, the polarity is being destabilized and central position is favored because of the IP ST. Still, quantitatively similar (slightly higher) single frequency and the GMR amplitude (slightly lower) are observed, Fig. 5.37a. The explanation of higher frequency values has been provided in previous subsection when discussing the comparison between $\left(b_{1}\right)$ to $(b)$. On the other hand, slightly lower output signal amplitude comes from higher mobility of the vortex in bottom layer, Fig. $5.37 \mathrm{~b}$, as compared $\left(\mathrm{j}_{1}\right)$ to $(\mathrm{j})$ because of replacing higher $\mathrm{M}_{\mathrm{s}}$ Co layer by lower $\mathrm{M}_{\mathrm{s}}$ Py layer. In $\left(\mathrm{j}_{1}\right)$ the relative separation of 
the vortices is smaller and, thus, the angles between the neighboring cells in top and bottom layer defining the amplitude of the output signal are lower.

Despite these quantitative similarities, still, a couple of qualitative differences shall be pointed out. First of all no higher harmonics are observed in the spectra as it was the case in $(\mathrm{j})$. Thus, the appearance of these side maxima modified the GMR amplitude so that highly non monotonic dependence with current was observed, open black squares Fig. 5.37a. In turn, $\left(\mathrm{j}_{1}\right)$ yields almost linear increase of the GMR amplitude with absolute current, open orange circles Fig. 5.37a. The linearity of this dependence arises from presence of one single frequency in the readout spectra throughout the whole current range. This in turn has to do with the fact that the threshold current for ST-driven dynamics depends on the material. Thus when both ferromagnetic layers are equal as in $\mathrm{Py} / \mathrm{Cu} / \mathrm{Py}$ valve only one threshold value is present. On the contrary, in $\mathrm{Co} / \mathrm{Cu} / \mathrm{Py}$ two different threshold, for Co and $\mathrm{Py}$, respectively, are to be found.

On the other hand, the increase of the GMR amplitude is traced back to the increase of the displacement of vortices in both layers, Fig. 5.37c, whereas it is essential that vortex in top layer remains in the vicinity of the PC. Interestingly, below the current of $-9 \mathrm{~mA}\left(\mathrm{j}_{1}\right)$ yields irregular behavior. On the contrary, (j) first led to the appearance of the critical current $(-15 \mathrm{~mA})$ at which the polarity switching in the top vortex was observed followed by some oscillations to finally support irregular core switching and associated spin wave radiation below that current. In other terms, recalling Fig. 5.17 below the critical current the polarity switch transformed (j) into (i). Similarly, one would expect such transformation between $\left(\mathrm{j}_{1}\right)$, Fig. 5.32 and analogous Fig. 5.28. Thus, no dynamics was observed in $\mathrm{Py} / \mathrm{Cu} / \mathrm{Py}$ when vortices of common polarities were considered, Fig. 5.28. Therefore, below the critical current of $-9 \mathrm{~mA}\left(\mathrm{j}_{1}\right)$ yields directly the irregular dynamics in both layers.

In the top layer vortex remains close to the $\mathrm{PC}$ and undergoes subsequent switching of the core polarity which is followed by the change of the gyration 
direction and spin wave radiation. In the bottom layer the vortex is found inside the $\mathrm{PC}$ oscillating with minor amplitude and triggering spin waves nucleation when changing the core polarity. As the current is further decreased $(-11 \mathrm{~mA})$ the vortex in the top layer preserves its negative polarity and is expelled far from the PC. Since the distance to the sample center is significant the vortex trajectory is not affected by the spin waves generated in the PC. No change in the behavior of vortex in the bottom layer is observed. Thus, considering to complexity of dynamics reported the results are only presented down to the critical current of $-9 \mathrm{~mA}$ where regular oscillations are still supported and no further study is carried out below $-11 \mathrm{~mA}$. Apart from that, the reason of observing lower critical current comparing $\mathrm{Py} / \mathrm{Cu} / \mathrm{Py}(-9 \mathrm{~mA})$ to $\mathrm{Co} / \mathrm{Cu} / \mathrm{Py}(-15 \mathrm{~mA})$ has been already thoroughly elaborated on in above subsections.

\subsection{Conclusions}

This vast study of interlayer coupled vortex pair dynamics brings about dozens of conclusions and confirms some features theoretically expected. For example it has been shown that the threshold current for vortex ST-driven dynamics in Py layer is lower than in Co layer and so does the critical current for the core polarity reversal. Vortex velocity increases with current as the consequence of ST linear increase with current. Thus, once the critical velocity is reached the core polarity switches.

In general, vortex polarity defines its gyration direction according to right hand rule. Thus, if common polarity of vortices is assumed in an asymmetric trilayer $\left(\mathrm{F}_{1} \neq \mathrm{F}_{2}\right)$ each vortex might move with its own eigenfrequency $\left(\mathrm{f}_{1}, \mathrm{f}_{2}\right)$ and the resulting output signal will represent the difference between these frequencies, $\mathrm{f}_{2}-\mathrm{f}_{1}$. Analogous, considering opposite polarity (opposite gyration direction) the resulting frequency shall be the sum of the eigenfrequencies. 


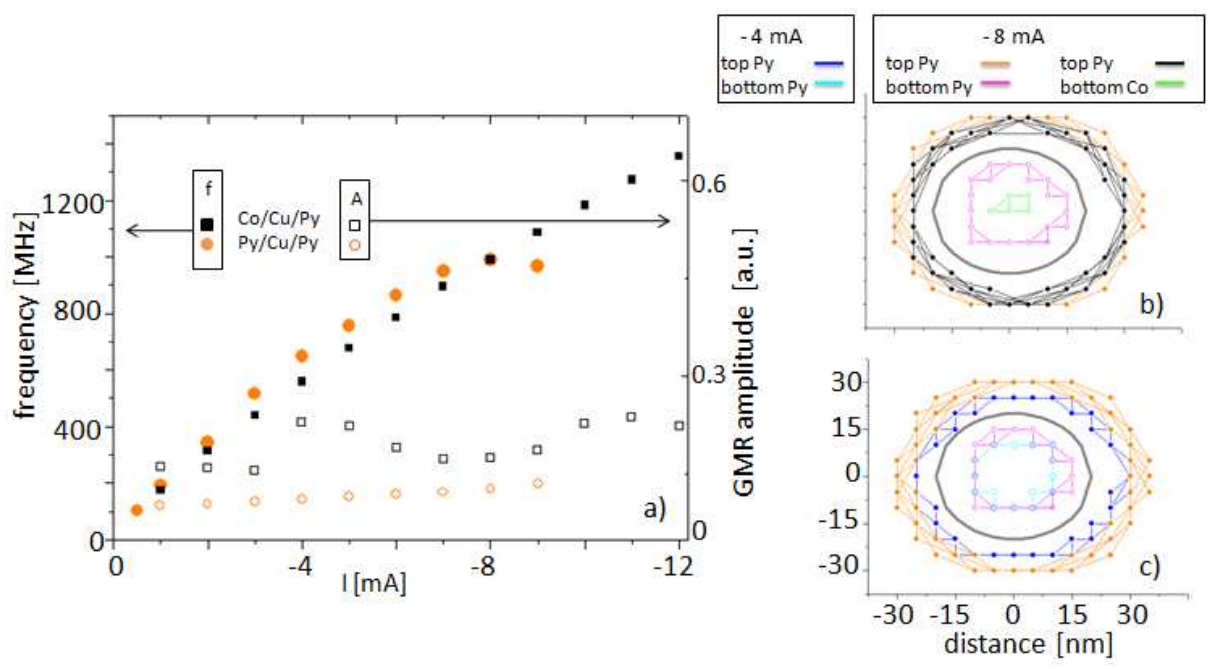

Fig. 5.37: ST active on both ferromagnetic layers. Frequency and GMR amplitude dependence on current, compared for the trilayers $\mathrm{Co} / \mathrm{Cu} / \mathrm{Py}$ in black and $\mathrm{Py} / \mathrm{Cu} / \mathrm{Py}$ in orange, (a). Comparison of vortex trajectory in top and bottom layers at the current of $-8 \mathrm{~mA}$ in case of symmetric and asymmetric structures, (b). Expansion of the orbits of both vortices with current in $\mathrm{Py} / \mathrm{Cu} / \mathrm{Py}$ structure, (c).

Furthermore, in case of opposite polarities one might observe vortex motion inside PC being superposition of the ccw and cw high and low frequency movements. The low frequency corresponds to the movement in the direction against right hand rule but magnetostatically coupled to the movement of the other vortex outside the PC. The high frequency movement, in turn, corresponds to a higher harmonic. Thus, the resulting output signal reflects the base frequency and its sum with the higher harmonics is observed.

The appearance of higher harmonics changes the output signal amplitude. Highest GMR amplitude has been observed in case of both vortices gyrating in phase outside the $\mathrm{PC}$.

Two vortices magnetostatically influence each other. This interaction scales with the distance and, thus, the frequency supported in the system is 
only affected by these interactions if the cores are close enough. Both polarity combinations: common and opposite are stable within some current range and lead to dynamic output except for a symmetric trilayer, where two high $\mathrm{M}_{\mathrm{s}}$ ferromagnetic layers are assumed. In the first case (common polarities) vortex orbit shows strong dependence on current, while in the second no such feature is observed. This is compatible with the latest model [80].

Interestingly, magnetostatic interactions might lead to vortex motion even in absence of ST. Provided that ST is active in one of the layers and vortex is triggered into motion, the time dependent changes of configuration give rise to energy flow into the layer where no ST is present. Thus, sustained oscillations of the vortex within might be observed. This energy feeding the vortex in the neighboring layer might even lead to the change of its polarity because of the steepening of the potential well with the increasing velocity of the ST driven vortex.

Hence, finally one concludes that the optimal situation i.e. highest output signal could be observed if in an asymmetric trilayer ST effect could be activated only on the high $\mathrm{M}_{\mathrm{s}}$ and blocked on the lower $\mathrm{M}_{\mathrm{s}}$ ferromagnetic layer. The electrons shall flow from the first to the second layer, respectively, and polarize by reflection while two vortices of common polarity are set as the initial state. This way, in high $\mathrm{M}_{\mathrm{s}}$ ferromagnet vortex is expelled from the $\mathrm{PC}$ via $\mathrm{ST}$ effect and the other vortex is easily driven into motion by the processes as described above. Blocking and enabling ST effect would have to be manipulated on the fabrication level when defining the interfaces. 


\section{COUPLED VORTICES DYNAMICS}

This chapter recompiles some preliminary work done considering a particular device, in which a $2 \times 2$ pseudo-matrix of PCs [14] has been fabricated and its response to SPC has been investigated. Lately it has been demonstrated experimentally that in such a structure it is possible to synchronize multiple STNOs. Similarly to the device studied in one of previous chapters, here the PCs were opened using the AFM nano-indentation technique [47], resulting in a non-flat profile for the upper ferromagnetic layer. Although very convincing evidence of phase-locking was provided in Ref. [14], the impact of this geometry on the dynamic properties of the system was not studied numerically. Hence, this chapter proceeds with the preliminary computational description of the system.

\subsection{Experimental findings}

In the sample, as presented in Fig. 6.1a, the distance between the contacts reads $500 \mathrm{~nm}$. However, arrays of different spacing (up to $10 \mu \mathrm{m}$ ) were also studied. Interestingly, in the samples with larger spacings few tens of mT external positive OP field had to be applied in order to sustain the output. Only the device with the interdistance of $500 \mathrm{~nm}$ exhibited behavior as presented in Fig. 6.1b, i.e. in absence of applied field a broad power spectrum was observed with four closely spaced peaks which merged into two and finally one peak as the current was increased. The reduction of the peak height in the reported spectra was accompanied by the increase of the output power. Therefore, this observation has been interpreted as a 
synchronization of multiple STNOs.

The authors argued that the magnetic configuration, allowing for the above observations, is composed of four vortices, nucleated at the contacts, four antivortices and an additional vortex in the middle of the sample. According to [14] such state can be nucleated and stabilized at the current of $40 \mathrm{~mA}$. Note, that all contacts are connected to common top and bottom electrodes. Therefore, the array can be seen as a parallel connected matrix of resistors, i.e. single contact can not be controlled independent. Authors further elaborated that lowest energy state in such a configuration requires that the neighboring V-AV exhibit opposite polarity. This, configuration was called the antiferromagnetic chessboard. Thus, its nucleation was only obtained in absence of any IP field. Already in presence of a field as low as $-1.2 \mathrm{mT}$ during the nucleation process led to destruction of the synchronization feature. Moreover, the latter was most efficient in terms of output power in absence of external field, so that any additional applied OP field caused a decrease in the output power peak, Fig. 6.1c. Before the acquisition of each spectrum a large $\mathrm{OP}$ reset field of $0.7 \mathrm{~T}$ was applied.

None of the ferromagnetic layers is exchange biased in the system and the dynamics was only observed for the current direction corresponding to electron flow from Co to Py layer. Thus, authors argued that $4 \mathrm{~nm}$ thick Py layer was the passive polarizing layer. In what follows the electrons reflected from $\mathrm{Au} / \mathrm{Py}$ interface impinging back on the $\mathrm{Au} / \mathrm{Co}$ interface exerted ST on the array of vortices and antivortices nucleated in $15 \mathrm{~nm}$ thick Co layer.

\subsection{Current and Oersted field distribution}

In order to define the active layer in the system first the exact distribution of current and the associated Oersted field must be known. For this calculation the commercial finite element software [75] has been employed and the whole 

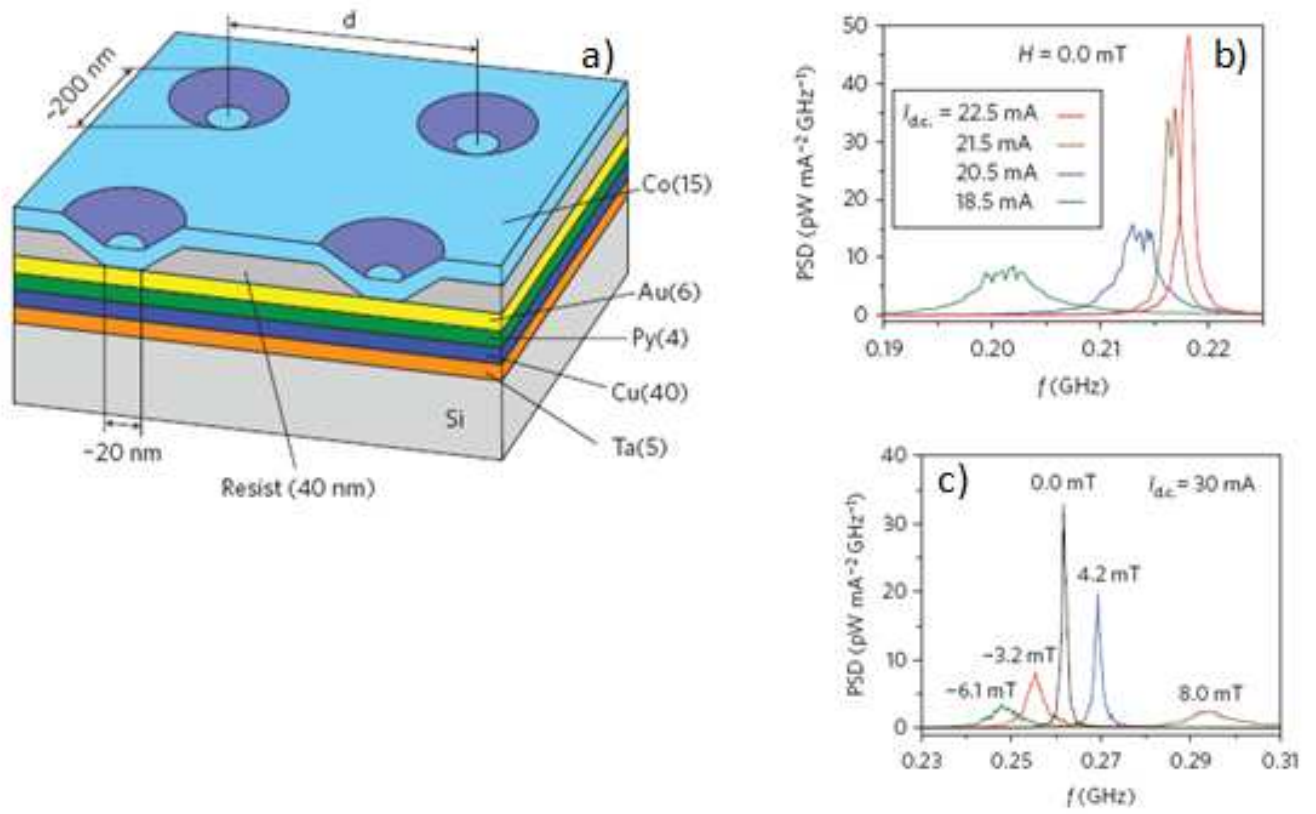

Fig. 6.1: The geometry of $2 \times 2$ pseudo-matrix of PCs, (a). Measured output power at different currents and in absence of external field, (b). The main peak amplitude at different applied OP fields [14], (c).

spin valve including the electrodes has been accounted for. Owing the $25^{\circ}$ inclination in the vicinity of all contacts, and based on the results from Chapter 4, one expects that highly nonuniform current distribution is going to be found across the sample. Indeed, the calculations reveal rather complicated profile of the current density in the structure, Fig. 6.2. Similarly to results obtained in Chapter 4 current distribution in Py layer is pretty uniform but in Co layer much more current flows at the edge of the contact rather than inside it. 


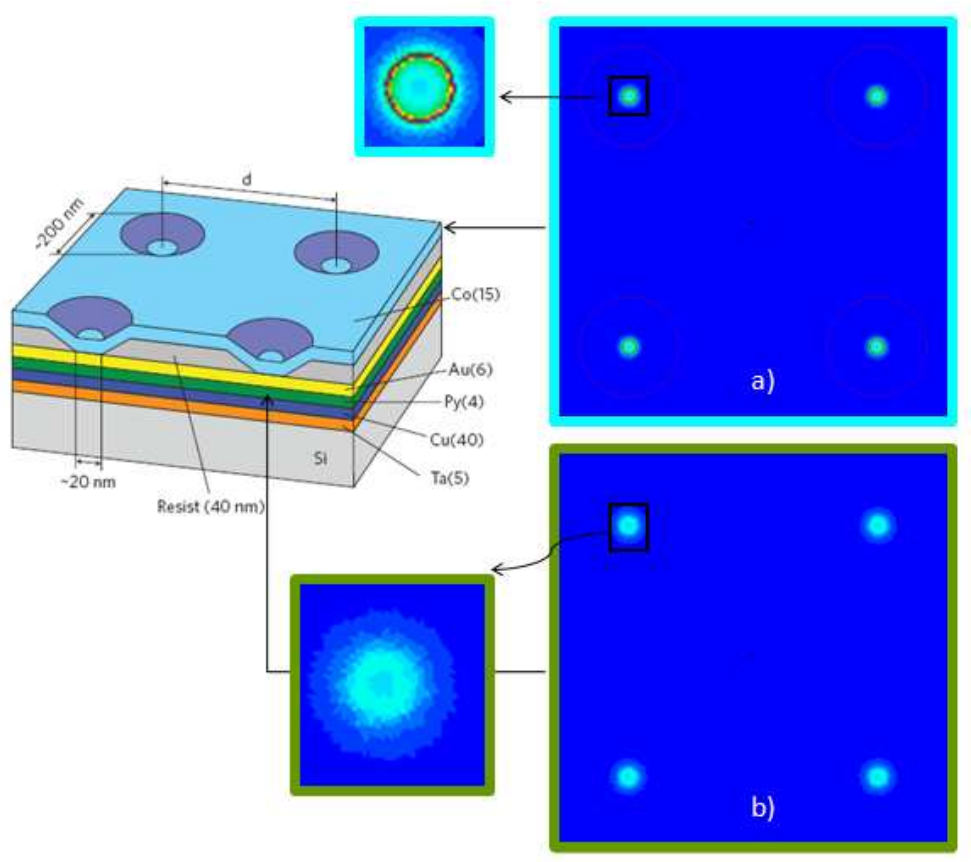

Fig. 6.2: Current distribution, as calculated with MagNet software [75], in the cross-section corresponding to Co (a) and Py (b) layer together with zoom close to one of the contacts.

The presence of an array of contacts complicates even more the distribution of the associated Oersted field. This field as a vectorial sum of the contributions from all four contacts breaks the circular symmetry. Again, analogous to results presented in Chapter 4 the maximum value of the Oersted field in Co layer is much larger than the corresponding maximum in Py layer, Fig. 6.3. Moreover, the above mentioned breaking of symmetry imposes that outside the square defined by the contacts the Oersted field is stronger than inside it. This asymmetric potential, in turn, shall influence the dynamics of the vortices. 


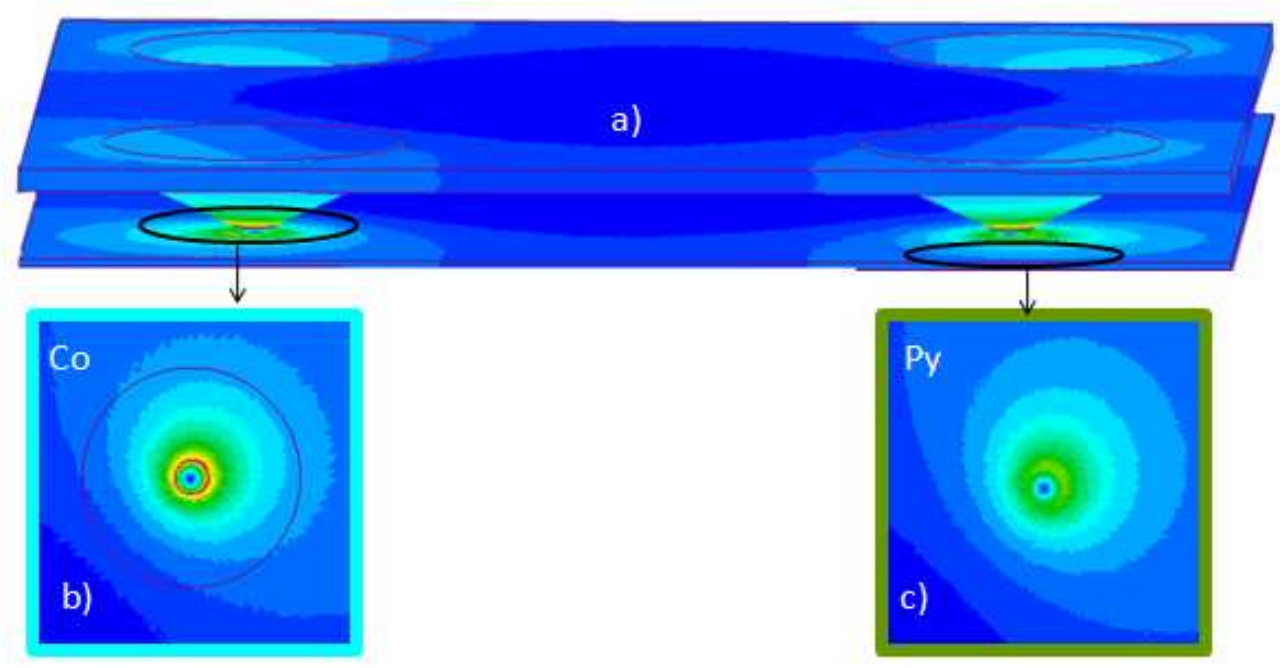

Fig. 6.3: Current associated Oersted field, as calculated with MagNet [75] software. Density plot as in tilted side view of the STT tilayer (a), in the crosssection corresponding to Co (b) and Py (c).

\subsection{Remarks}

The next step would be to introduce the result of the above presented calculations into the full micromagnetic study. In analogy to Chapter 4, first the dynamical part of the valve shall be identified. Hence, the free and the reference layer should be defined. This task, considering the complexity of the geometry and the size of the computational area required for the calculations are far beyond the time frame of this work. Thus, the investigation of coupling mechanism in arrays of PCs remains reserved for the future study. 


\section{SUMMARY AND OUTLOOK}

The work presented here was focussed on the computational analysis of dynamics supported in STT devices. The discovery of spin transfer itself is rather recent $[6,7]$. Thus, even though it has been one of the most intensively studied subjects in solid state magnetism over the last decade the understanding of numerous mechanism is still far from being complete. One of feasible future applications of STT requires the development of a reliable STNO. Particularly interesting are the oscillators working in absence of external field. An example of such device was investigated within this work where in presence of a nonstandard ST profile the oscillations were sustained even without any applied field. Remarkably, the design of the structure, i.e. materials, thickness etc. has a great impact on electron transport properties. Within this work, a numerical solution based on the theoretical model [16] was developed for the determination of the STT angular dependence. This allowed for prediction of interesting behavior in nonstandard, asymmetric trilayers. Results of systematic micromagnetic study yielded an excellent quantitative agreement with the experimental findings [17]. Interestingly, this work demonstrated that the dynamics which was believed to be driven only by the STT in reality was also based on the presence of the magnetostatic contribution from the pinned layer.

Later, a vortex mode has been investigated. Again the main motivation behind the study was providing the explanation for the experimentally observed features. Once more an excellent match between the numeric and experimental results was obtained, thus revealing a novel way of tailoring vor- 
tex based STNO. It has been demonstrated that the dynamics of a magnetic vortex in the free layer might be determined by the design of the pinned layer geometry. This discovery added important insights into, still poorly known, vortex dynamics in PC devices.

Hence, the main obstacle on the way to successful application of vortex based STNO is the output power and linewidth. An interesting solution to the problem of linewidth is the synchronization of two vortices nucleated one in each ferromagnetic layer in a standard spin valve with no exchange bias. The computational study of linewidths in point contact devices would be very demanding and particularly time taking but the determination of qualitative of dynamics in such systems is perfectly within possibilities of state-of-the art computations. Thus, within this work a vast study of interlayer coupled vortex based STNO has been performed. The definition of the ST active layer in the sample and choice of core polarities has been demonstrated to have great impact on the behavior of the system. In particular, optimal conditions for the improvement of output signal amplitude have been defined. These results will be highly valuable for the future desgin of vortex based interlayer coupled STNO.

The examples studied within this work required application of micromagnetic modeling technique. In particular, point contact geometry and vortex mode can only be investigated numerically using micromagnetic framework since single spin model does not allow for implementation of spatially nonuniform states. Considering all the methodological issues involved and long computational times systematic micromagnetic studies of point contact devices are rather rare in the literature even though often, considering sample geometry, the system description is beyond the approximations of theoretical models. Thus, only computational study can reveal the nature of experimental findings. 


\section{BIBLIOGRAPHY}

[1] IBM RD 50-1; Spintronics; A retrospective and perspective. http://www.research.ibm.com/journal/rd/501/wolf.html.

[2] Stuart Wolf. Physics Profile: "Stu Wolf: True D! Hollywood Story".

[3] P.M. Tedrow; R. Meservey. Phys. Rev. Lett., 26, 192, 1971.

[4] M. Julliere. Phys. Lett. A, 54, 225-226, 1975.

[5] M. Johnson; R.H. Silsbee. Phys. Rev. Lett., 55, 1790, 1985.

[6] J.C. Slonczewski. J. Magn. Magn. Mater., 159, 1996.

[7] L. Berger. Phys. Rev. B, 54, 9353, 1996.

[8] M. AlHajDarwish; H. Kurt; S. Urazhdin. Phys. Rev. Lett., 93, 157203, 2004.

[9] M. Tsoi; J.Z. Sun; M.J. Rooks. Phys. Rev. B., 69, 100406, 2004.

[10] A.N. Slavin; V.S. Tiberkevich. Phys. Rev. B, 72, 094428, 2005.

[11] S.I. Kiselev; J.C. Sankey; I.N. Krivorotov; N.C. Emley; R.J. Schoelekopf; R.A. Buhrman. Nature, 425, 2003.

[12] S. Kaka; M.R. Pufall; W.H. Rippard; T.J.Silva; S.E. Rusek; J.A. Katine. Nature, 437, 389, 2005.

[13] F.B. Mancoff; N.D. Rizzo; B.N. Engel; S. Tehrani. Nature, 437, 393, 2005. 
[14] A. Ruotolo; V. Cros; B. Georges; A. Dussaux; J. Grollier; C. Deranlot; R. Guillemet; K. Bouzehouane; S. Fusil; A. Fert. Nat. Nanotech., 4, 528 $-532,2009$.

[15] N. Locatelli; V.V. Naletov; J. Grollier; G. de Loubens; V. Cros; C. Deranlot; C. Ulusse; G. Faini; O. Klein; A. Fert. cond-mat.mtrl-sci, arXiv:1005.0290v2, 2010.

[16] J. Barnaś; A. Fert; M. Gmitra; I. Weymann; V. Dugaev. Phys. Rev. B, 72, 024426, 2005.

[17] O. Boulle; V. Cros; J. Grollier; L.G. Pereira; C. Deranlot; F. Petroff; G. Faini; J. Barnaś; A. Fert. Nature Phys., 3, 2007.

[18] O. Boulle; V. Cros; J. Grollier; L.G. Pereira; C. Deranlot; F. Petroff; G. Faini; J. Barnaś; A. Fert. Phys. Rev. B, 77, 174403, 2008.

[19] T. Devolder; J.-V. Kim; P. Crozat; C. Chappert; M. Manfrini; M. van Kampen; W. van Roy; L. Lagae; G. Hrack; T. Schrefl. App. Phys. Lett., 95, 012507, 2009.

[20] M. Gmitra; J. Barnaś. Phys. Rev. B, 79, 012403, 2009.

[21] T. Valet; A. Fert. Phys. Rev. B, 48, 10, 1993.

[22] J.A. Katine; F.J. Albert; R.A. Buhrman; E.B. Myers; D.C. Ralph. Phys. Rev. Lett., 84, 3149, 2000.

[23] I.N. Krivorotov; N.C. Emley; J.C. Sankey; S.I. Kiselev; D.C. Ralph; R.A. Buhrman. Science, 307, 2005.

[24] I.N. Krivorotov; N.C. Emley; R.A. Buhrman; D.C. Ralph. Phys. Rev. $B, 77,054440,2008$.

[25] I.N. Krivorotov; D.V. Berkov; N.L. Gorn; N.C. Emley; J.C. Sankey; S.I. Kiselev; D.C. Ralph; R.A. Buhrman. Phys. Rev. B, 76, 024418, 2007. 
[26] S.I. Kiselev; J.C. Sankey; I.N. Krivorotov; N.C. Emley; M. Rinkoski; C. Perez; R.A. Buhrman; D.C. Ralph. Phys. Rev. Lett., 93, 3, 2004.

[27] M. Gmitra und J. Barnaś. App. Phys. Lett., 89, 223121, 2006.

[28] M. Gmitra und J. Barnaś. Phys. Rev. Lett., 99, 097205, 2007.

[29] P. Baláž; M. Gmitra; J. Barnaś. Acta Phys. Pol., 115, 2008.

[30] P. Baláž; M. Gmitra; J. Barnaś. Phys. Rev. B, 79, 144301, 2009.

[31] E. Jaromirska; P. Baláž; L. Lopez-Diaz; J. Barnaś. J. App. Phys., 106, 113909, 2009.

[32] E. Jaromirska; P. Baláž; L. Lopez-Diaz; J. Barnaś. Phys. Rev. B, 81, 014408, 2010.

[33] W.F. Brown. Micromagnetism. Wiley, New York, 1963.

[34] D.V. Berkov; J. Miltat. J. Magn. Magn. Mat., 320, 7, 2008.

[35] D.V. Berkov; N.L. Gorn. Phys. Rev. B, 71, 052403, 2005.

[36] D.V. Berkov; N.L. Gorn. Phys. Rev. B, 72, 094401, 2005.

[37] M.R. Pufall; W.H. Rippard; M.L. Schneider; S.E Russek. Phys. Rev. B, 75, 140404(R), 2007.

[38] V.S. Pribiag; I.N. Krivorotov; G.D. Fuchs; P.M. Braganca; O. Ozatay; J.S. Sankey; D.C. Ralph; R.A. Buhrman. Nat. Phys., 3, 498-503, 2007.

[39] Q. Mistral; M. van Kampen; G. Hrkac; Joo-Von Kim; T. Devolder; P. Crozat; C. Chappert; L. Lagae; T. Schrefl. Phys. Rev. Lett., 100, 257201, 2008.

[40] K.Yu. Guslienko; B.A. Ivanov; V. Novosad; Y. Otani; H. Shima; K. Fukamichi. J. App. Phys., 91, 10, 2002. 
[41] A.V. Khvalkovskiy; J. Grollier; A. Dussaux; K. A. Zvezdin; V. Cros. Phys. Rev. B, 80, 0140401(R), 2009.

[42] V.S. Pribiag; G. Finocchio; B.J. Williams; D.C. Ralph; R.A. Buhrman. Phys. Rev. B, 80, 180411(R), 2009.

[43] A. Dussaux; B. Georges; J. Grollier; V. Cros; A.V. Khvalkovskiy; A. Fukushima; M. Konoto; H. Kubota; K. Yakushiji; S. Yuasa; K.A. Zvezdin; K. Ando; A. Fert. Nat. Com., 1, 8, 2010.

[44] A.V. Khvalkovskiy; J. Grollier; N. Locatelli; Ya.V. Gorbunov; K. A. Zvezdin; V. Cros. App. Phys. Lett., 96, 212507, 2010.

[45] M. Bode; A. Wachowiak; J. Wiebe; A. Kubetzka; M. Morgenstern; R. Wiesendanger. App. Phys. Lett., 86, 948, 2004.

[46] G. Finocchio; V.S. Pribiag; L. Torres; R.A. Buhrman; B. Azzerboni. App. Phys. Lett., 96, 102508, 2010.

[47] K. Bouzehouane; S. Fusil; M. Bibes; J. Carrey; T. Blon; M. Le Du; P. Seneor; V. Cros; L. Vila. Nano Lett., 3, 1599-1602, 2003.

[48] Wolfram Research. www.wolfram.com.

[49] J. Miltat. 83, Topics of App. Phys. 1993.

[50] A. Brataas; G.E.W. Bauer; P.J. Kelly. Phys. Rep., 427, 2006.

[51] A. Brataas; Yu. V. Nazarov; G.E.W. Bauer. Eur. Phys. J. B, 22, 99, 2001.

[52] M.D. Stiles; A. Zangwill. J. App. Phys, 91, 6812, 2002.

[53] J. Barnaś; A. Fert; M. Gmitra; I. Weymann; V. Dugaev. Mat. Sci. Eng. $B, \mathbf{1 2 6}, 2006$. 
[54] D.C. Ralph; M.D. Stiles. J. Magn. Magn. Mat., 2008.

[55] M. Donahue J. Miltat. Handbook of magnetism and advanced magnetic materials, Band 2. Wiley, Chichester, 2007.

[56] A. Aharoni. J. App. Phys., 1998.

[57] W.H. Press; S.A. Teukolky; W.T. Watterling; B.P. Flannery. Numerical Recipes in Fortran: The Art of Computing. 1996.

[58] A.J. Newell; W. Williams; D.J. Dunlop. J. Geophys. Res., 1993.

[59] M.F. Lai; Z.H. Wei; Ch.R. Chang; N.A. Usov; J.C. Wu; J.Y. Lai. J. Magn. Magn. Mat., 282, 2004.

[60] D. Scholl; M. Donath; D. Mauri; E. Kay. Phys. Rev. B, 43, 16, 1991.

[61] R.D. McMichael; M.D. Stiles. J. App. Phys., 97, 10J901, 2005.

[62] M. Grimsditch; G.K. Leaf; H.G. Kaper; D. Karpeev; R.E. Camley. Phys. Rev. B, 69, 174428, 2004.

[63] L. Torres; G. Finocchio; L. López Díaz; E. Martinez; M. Carpentineri; G. Consolo; B. Azzerboni. J. App. Phys., 101, 09A502, 2007.

[64] L. Torres; L. López Díaz; E. Martinez; G. Finocchio; M. Carpentineri; B. Azzerboni. J. App. Phys., 101, 053914, 2007.

[65] E. Jaromirska; L. Lopez-Diaz; A. Ruotolo; J. Grollier; V. Cros; D. Berkov. submitted to PRB, 2010.

[66] W.H. Rippard; M.R. Pufall; S. Kaka; S.E. Russek; T.J. Silva. Phys. Rev. Lett., 92, 027201, 2004.

[67] D.V. Berkov; N.L. Gorn. J. App. Phys., 99, 08Q701, 2006. 
[68] G. Consolo; L. Lopez-Diaz; L. Torres; B. Azzerboni. Phys. Rev. B, 75, 214428, 2007.

[69] K.Yu. Guslienko; K.S. Bychanan; S.D Bader; V. Novosad. App. Phys. Lett., 86, 223112, 2005.

[70] K.Yu. Guslienko; A.N. Slavin; V. Tiberkevich; S-K. Kim. Phys. Rev. Lett., 101, 247203, 2008.

[71] K.Yu. Guslienko; X.F Han; D.J. Keavney; R. Divan; S.D. Bader. Phys. Rev. Lett., 96, 0671205, 2006.

[72] M.D. Stiles; A. Zangwill. Phys. Rev. B, 66, 014407, 2002.

[73] www.math.nist.gov/oommf/. OOMMF - Object Oriented Micromagnetic Framework.

[74] K.S. Buchanan; K.Yu. Guslienko; S.-B. Choe; A. Doran; A. Scholl; S.D. Bader; V. Novosad. J. App. Phys, 97, 10H503, 2005.

[75] www.infolityca.com. MagNet commercial software.

[76] T. Devolder; J.-V. Kim; M. Manfrini; W. van Roy; L. Lagae; C. Chappert. App. Phys. Lett, 97, 072512, 2010.

[77] A. Wachowiak; J. Wiebe; M. Bode; O. Pietzsch; M. Morgenstern; R. Wiesendanger. Science, 298, 577-580, 2002.

[78] G. de Loubens; A. Riegler; B. Pigeau; F. Lochner; F. Boust; K.Y. Guslienko; H. Hurdequint; L.W. Molenkamp; G. Schmidt; A.N. Slavin; V.S. Tiberkevich; N. Vukadinovic; O. Klein. Phys. Rev. Lett., 102, 177602, 2009.

[79] D.V. Berkov; N.L. Gorn. Phys. Rev. B, 80, 064409, 2009.

[80] E. Jaromirska; S. Komineas; L. Lopez-Diaz. in preparation, 2010. 


\section{ACKNOWLEDGMENTS}

This work is a result of a lot of team working. In the first place I would like to acknowledge the team coach, my supervisor Luis López Díaz for his everlasting patience with me. The rest of the team: Luis Torres, David Aurélio and Javier Gómez for being the best team ever. Main financial support was provided by European Training Network Spinswitch under contract number MRTN-CT-2006-035327. However, Spanish government grant MAT2008-04 706/NAN and Junta de Castilla y Leon project SA 025A08 are also acknowledged.

In different parts of my $\mathrm{PhD}$ I was supported by several people, some of whom contributed significantly to the results presented within this work. I would like to mention in particular Prof. Józef Barnaś here. Jacques Miltat, Stavros Komineas, Giancarlo Consolo, Pilar Garcia provided administrative as well as scientific assistance throughout the last three years making my PhD struggle successful.

For all professional and personal contribution in this period I would like to acknowledge Pavel Baláž and Mauricio Manfrini. Dimitri Berkov, for all the time he has invested in monitoring the progress of the results but especially for his attitude that made me believe in science some times.

I would also like to include acknowledgments referring to the part of life that excludes science. My cooking quartet: Ana, Erick, David, Javi for all the master dinner symphonies. Enrique for 'todos los pegues' and 'apretones a muerte'. Jacinta, Antonio, Toni and Pedro for being my second family. There are no words I could express my appreciation to 'Rellenitos': Alfonso 
and Noemi whose existence makes me see things so much different.

Finally, I would like to thank Raúl who has always stayed firm at my side no matter how annoying I could have been. 